\title{
Model-based Experimental Analysis of Enzyme Kinetics in Aqueous- Organic Biphasic Systems
}

Von der Fakultät für Maschinenwesen der Rheinisch-Westfälischen Technischen Hochschule Aachen zur Erlangung des akademischen Grades eines Doktors der Ingenieurwissenschaften genehmigte Dissertation

vorgelegt von

\section{Michael Zavrel}

Berichter: Universitätsprofessor Dr.-Ing. Jochen Büchs Universitätsprofessor Dr.-Ing. Wolfgang Marquardt

Tag der mündlichen Prüfung: $\quad$ 26. Oktober 2009

Diese Dissertation ist auf den Internetseiten der Hochschulbibliothek online verfügbar. 


\title{
Model-based Experimental Analysis of Enzyme Kinetics in Aqueous- Organic Biphasic Systems
}

\author{
Michael Zavrel
}




\section{Vorwort}

Die vorliegende Arbeit entstand während meiner Tätigkeit als wissenschaftlicher Mitarbeiter am Lehrstuhl für Bioverfahrenstechnik innerhalb der Aachener Verfahrenstechnik der RWTH Aachen. Dieses Forschungsprojekt war eingegliedert in den Sonderforschungsbereich 540 "Modellgestützte experimentelle Analyse kinetischer Phänomene in mehrphasigen Reaktionssystemen" und in das Graduiertenkolleg GK 1166 "BioNoCo - Biokatalyse in unkonventionellen Medien".

Mein besonderer Dank gilt Dr. Antje Spiess und Professor Jochen Büchs für die Betreuung dieser Arbeit und für die Möglichkeit am Lehrstuhl für Bioverfahrenstechnik promovieren zu dürfen. Ihre Unterstützung, ihre Anregungen und ihre Offenheit gegenüber neuen Ideen waren Voraussetzung für das Gelingen dieser Arbeit. Mein Dank gilt auch Professor Wolfgang Marquardt für die Übernahme des Koreferates und für seine nützlichen Anregungen während meiner Promotion, sowie Professor Martina Pohl und Professor Reinhold Kneer für die Übernahme des Prüfungsbeisitzes bzw. des Prüfungsvorsitzes.

Von wesentlicher Bedeutung für die Bearbeitung des stark interdisziplinären Themas war die enge Zusammenarbeit innerhalb des Sonderforschungsbereichs und des Graduiertenkollegs. Herausheben möchte ich dabei meine direkten Kooperationspartner Claas Michalik (AVT Lehrstuhl für Prozesstechnik), Thomas Schmidt (Lehrstuhl für Biotechnologie) und Tilman Schwendt (Fraunhofer Institut für Lasertechnik), denen ich für die konstruktiven Diskussionen und die gewinnbringende Zusammenarbeit herzlich danke. Gleiches gilt für Martina Peters und Dr. Lasse Greiner (Institut für Technische und Makromolekulare Chemie), Professor Marion Ansorge-Schumacher (Lehrstuhl für Biotechnologie), sowie Mariya Kokova und Melanie Schwarz (Institut für Molekulare Enzymtechnologie, Heinrich-Heine Universität Düsseldorf).

Nicht zu vergessen sind meine Kollegen am Lehrstuhl für Bioverfahrenstechnik. Ihnen danke ich für die angenehme Arbeitsatmosphäre und für die freundschaftliche Verbundenheit auch außerhalb des Lehrstuhls. Für die Korrekturen einzelner Passagen der Arbeit danke ich 
Kerasina Dimoula, Lars Regestein, Matthias Funke, Gernot Jäger, Gregor Steinhorn, Philip Engel, Sven Hansen, Claas Michalik und Martina Peters.

Die Leistungen der betreuten Studenten möchte ich nicht unerwähnt lassen, da sie mich durch ihre Arbeiten wesentlich unterstützt haben und wertvolle Ideen einfließen ließen. Ich danke Ana Luisa Nunes de Almeida, Marcus Verhülsdonk, Eddy Santosa, Patrick Gillessen, Christoph Kuhlen, Bertrand Guelat, Jens Kahlen, Andrew Choi, Khairul Azman Rahmat, Daniela Bross, Karl Kochanowski, Christian Pohlmann, Martin Pöhnlein, Irene Somoza, Praveen Kumar, Kai Wetzel, Patrick Schmidt, Sebastian Recker und Manuel Dahmen.

Danken möchte ich schließlich meinen Eltern für die Unterstützung während meines Studiums und Auslandsaufenthaltes, sowie meiner Schwester und meinem Schwager für die Durchsicht der Arbeit. Mein größter Dank gebührt meiner Frau Cindy, die mir trotz der jahrelangen Ferne während der Promotion stets den notwendigen Rückhalt gegeben hat. 
Causarum enim cognitio cognitionem eventorum facit.

(Die Kenntnis der Ursachen bewirkt die Erkenntnis der Ergebnisse.)

Marcus Tullius Cicero (106 - 43 v. Chr.) - Topica 67 


\section{Kurzfassung}

\section{Modellgestützte experimentelle Analyse von Enzymkinetiken in wässrig-organischen Zweiphasensystemen}

Die Immobilisierung von Biokatalysatoren in Hydrogelkugeln, die in einem organischen Lösungsmittel suspendiert werden, ist eine vielversprechende Methode zur Produktion hydrophober Feinchemikalien. Die rationale Entwicklung solcher Immobilisate wird jedoch aufgrund der Überlagerung von Stofftransfer, Diffusion und Enzymreaktion erschwert. Deshalb wurde ein mechanistisches Kinetikmodell entwickelt, das diese Phänomene quantitativ beschreibt. Als Beispiel wurde die stereoselektive Carboligation zweier 3,5Dimethoxybenzaldehyd-Moleküle (DMBA) zu $(R)-3,3$ ',5,5'-Tetramethoxybenzoin (TMB) mit Hilfe des Enzyms Benzaldehydlyase (BAL) untersucht. Dabei wird die BAL in $\kappa$-Carrageenan-Hydrogelkugeln immobilisiert, welche von einem organischen Lösungsmittel umgeben sind.

Zunächst wurden die Phänomene Enzymreaktion, Stofftransfer und Diffusion separat untersucht. Die dabei entwickelten Kinetikmodelle wurden schrittweise miteinander kombiniert und schließlich zu einem Gesamtmodell zusammengefasst. Zur Untersuchung jedes einzelnen Reaktionssystems wurde die modellgestützte experimentelle Analyse (MEXA-Methode) angewendet, die a priori Simulationen, Sensitivitätsanalysen und optimale Versuchsplanung einschließt. Damit wird nicht nur die Entwicklung mechanistischer Kinetikmodelle, sondern auch die Entwicklung optimaler Messmethoden und -techniken unterstützt.

Mit Hilfe der MEXA-Methode konnten sowohl die kinetischen Parameter mit hoher Genauigkeit geschätzt, als auch neue mechanistische Kinetikmodelle entwickelt werden, die mögliche Limitationen und Engpässe aufdecken. Beispielsweise wurde die Freisetzung des Produktes als geschwindigkeitsbestimmender Schritt im Katalysemechanismus der BAL identifiziert, während die Anlagerung der Substrate den katalytischen Engpass bei der Benzoylformiatdecarboxylase (BFD) darstellt. Darüber hinaus wurde ein neuer Ansatz zur Auswahl eines geeigneten Lösungsmittels entwickelt und angewendet. Dieser zeigte, dass Methylisobutylketon hohe Produktausbeuten für die untersuchte Carboligation ermöglicht. 
Schließlich wurde die Diffusion der Reaktanden im Hydrogel untersucht. Hierbei ergaben a priori Simulationen, dass der Kugelmittelpunkt den optimalen Messort darstellt. Zudem konnte gezeigt werden, dass das Diffusionsgesetz nach Nernst und Planck anstelle des Fick'schen Gesetzes für die Modellierung der Diffusion dissoziierender Stoffe wie Propionsäure in Hydrogelkugeln angewendet werden muss.

Die Kopplung dieser einzelnen Systeme offenbarte, dass eine modellgestützte Versuchsplanung notwendig ist um Limitationen zu vermeiden. Zum Beispiel, sind in einem gerührten Zweiphasensystem die enzymkinetischen Parameter nur dann identifizierbar, wenn die Reaktion geschwindigkeitsbestimmend ist. Außerdem sind hierbei Messungen sowohl in der wässrigen als auch organischen Phase erforderlich. Das Emulgieren des Zweiphasensystems sollte vermieden werden, da hierbei die Bildung von Aggregaten beobachtet wurde, die auf eine Grenzflächeninaktivierung der Enzyme hindeutet.

Zuletzt wurden die erhaltenen Parameterwerte für das Gesamtsystem und für die gekoppelten Systeme mit denen aus den einzelnen Stoffsystemen verglichen. Dabei konnten für die enzymkinetischen Parameter, sowie für die Partitionskoeffizienten und für den Diffusionskoeffizienten des Substrats keine signifikanten Abhängigkeiten von den jeweiligen Stoffsystemen festgestellt werden. Allerdings weisen die Stofftransportkoeffizienten und der Diffusionskoeffizient des Produkts für die verschiedenen Stoffsysteme deutliche Unterschiede auf. Aus diesem Grund müssen zur optimalen Gestaltung von Enzymimmobilisaten die Modellparameter am Gesamtsystem bestimmt werden, wohingegen die Verwendung der in den einzelnen Stoffsystemen separat bestimmten Parameterwerte zu Fehlern führen kann.

Mit Hilfe des entwickelten mechanistischen Modells für das gelstabilisierte Zweiphasensystem können Limitierungen durch Diffusion und Stofftransfer erkannt werden. Somit stellt dieses den Grundstein für die modellgestützte Entwicklung von Enzymimmobilisaten dar und ermöglicht die Optimierung derartiger Prozesse. $\mathrm{Zu}$ diesem Zweck kann das Modell an andere Reaktionen, Biokatalysatoren, Lösungsmittel und Geometrien der Immobilisate angepasst werden. Die durch die mechanistische Modellierung identifizierten Engpässe im Katalysemechanismus der Enzyme BAL und BFD werden sicherlich zur Entwicklung neuer und aktiverer Enzymvarianten beitragen. 


\section{Abstract}

Immobilization of biocatalysts in hydrogel beads which are suspended in organic solvents is a promising approach for the production of hydrophobic fine chemicals. Due to the superposition of mass transfer, diffusion, and enzyme reaction the rational design of such immobilizates is rather complex. For this reason, a mechanistic kinetic model considering all three phenomena was derived. As an example, the stereoselective carboligation of two 3,5dimethoxy-benzaldehyde (DMBA) molecules to (R)-3,3',5,5'-tetramethoxy-benzoin (TMB) catalyzed by the enzyme benzaldehyde lyase (BAL) was investigated. BAL was immobilized in $\kappa$-carrageenan hydrogel beads which were surrounded by an organic solvent.

In the first step the phenomena enzyme reaction, mass transfer, and diffusion were studied in separate systems. Each system was individually investigated using the model-based experimental analysis (MEXA) approach including a priori simulations, sensitivity analysis, and optimal experimental design. This supports not only the development of mechanistic kinetic models, but also the determination of optimal measurement methods and the development of new experimental setups. Subsequently, the derived individual kinetic models were successively coupled and finally combined to a kinetic model for the gel-stabilized aqueous-organic biphasic reaction system.

Using the MEXA approach, not only the kinetic parameters could be estimated with high precision, but also new mechanistic models could be developed which revealed possible limitations and bottlenecks. Accordingly, the release of the product was identified as ratelimiting step in the enzymatic mechanism of BAL, whereas the binding of the substrate turned out as the catalytic bottleneck in the mechanism of benzoylformate decarboxylase (BFD). Furthermore, a new approach for solvent selection was developed in order to optimize the extractable yield of the carboligation reaction in a biphasic system. Consequently, methyl-isobutyl-ketone was chosen as organic solvent. The diffusion of the reactants in the hydrogel bead was investigated in the bead center, which was identified as the optimal measurement position using a priori simulations. It was demonstrated on the example of propionic acid that Nernst-Planck law is superior to Fick's law for modeling the diffusion of dissociating species in hydrogel beads. 
The coupling of the sub-systems revealed that sophisticated experimental design is crucial to avoid limitations due to the superposition of the phenomena. For instance, in a stirred biphasic system the enzyme kinetic parameters can only be identified if the reaction is rate-limiting. Furthermore, measurements in both phases are required. Emulsification should be avoided since the formation of aggregates was observed which indicates enzyme precipitation due to phase toxicity.

Finally, the parameter estimates for the gel-stabilized aqueous-organic biphasic reaction system were compared to those obtained for the individual and coupled systems. No significant influence of the reaction system on the enzyme kinetic parameters, on the partition coefficients, and on the effective diffusion coefficient of the substrate could be observed. However, the estimated values of the mass transfer coefficients and the effective diffusion coefficient of the product deviated from system to system. Therefore, for the rational design of enzyme immobilizates the model parameters have to be determined using the complete system. Adopting the parameter estimates obtained from individual systems may lead to an incorrect model prediction and an inefficient process design.

The derived mechanistic kinetic model for the gel-stabilized aqueous-organic system allows to detect limitations caused by diffusion or mass transfer, and paves the way for the rational design of enzyme immobilizates and for the optimization of such processes. It can easily be adapted to other reaction systems, biocatalysts, solvents, and geometries of immobilizates. Moreover, the identification of catalytic bottlenecks in the enzyme mechanisms of BAL and BFD will certainly support the development of new enzyme variants with enhanced activities. 


\section{Contents}

Nomenclature $\quad$ VII

$\begin{array}{ll}\text { Figures } & \text { XIV }\end{array}$

$\begin{array}{lll}\text { Tables } & \text { XXIV }\end{array}$

1 Introduction 1

1.1 Biocatalytic production of hydrophobic fine chemicals $\quad 1$

1.2 Enzyme reactions using non-conventional media 2

1.3 Mechanistic modeling of enzyme immobilizates 4

1.4 Model-based experimental analysis 5

1.4.1 Mechanistic kinetic modeling and preliminary analysis 6

1.4.2 Optimal experimental design and measurement techniques $\quad 7$

1.4.3 Solution of inverse problems 9

$\begin{array}{lll}1.5 & \text { Interdisciplinary collaboration }\end{array}$

$\begin{array}{lll}1.6 & \text { Objectives and overview } & 10\end{array}$

\section{Enzyme Kinetics in Homogeneous Aqueous Phase}

2.1 Comparison of different approaches and computer programs for progress curve analysis of enzyme kinetics

2.1.1 Introduction

2.1.2 Materials and methods

2.1.2.1 Experimental 15

2.1.2.2 Kinetic model 16

2.1.2.3 In silico experiments $\quad 17$

2.1.2.4 Influence of the amount of data 17

$\begin{array}{ll}\text { 2.1.2.5 Differentiation of concentration data } & 17\end{array}$

$\begin{array}{lll}\text { 2.1.2.6 Computer programs } & 18\end{array}$ 
$\begin{array}{lll}2.1 .3 & \text { Results and discussion } & 20\end{array}$

$\begin{array}{lll}\text { 2.1.3.1 Progress curves } & 20\end{array}$

2.1.3.2 Differentiation of concentration data 21

$\begin{array}{ll}\text { 2.1.3.3 Comparison of computer programs } & 24\end{array}$

$\begin{array}{lll}2.1 .4 & \text { Conclusions } & 28\end{array}$

2.2 Mechanistic kinetic model for benzaldehyde lyase 28

$\begin{array}{lll}2.2 .1 & \text { Introduction } & 28\end{array}$

2.2.2 Materials and methods 31

2.2.2.1 Experimental 31

2.2.2.2 Model identification approach $\quad 32$

$\begin{array}{lll}2.2 .3 & \text { Results and discussion } & 34\end{array}$

2.2.3.1 Derivation of the mechanistic kinetic model 34

$\begin{array}{lll}2.2 .3 .2 & \text { Parameter estimation } & 38\end{array}$

2.2.3.3 Model revision $\quad 45$

$\begin{array}{lll}2.2 .4 & \text { Conclusions } & 52\end{array}$

2.3 Kinetic modeling of benzaldehyde lyase with micro-reaction rate constants $\quad 52$

2.3.1 Introduction $\quad 52$

2.3.2 Micro-kinetic model 53

2.3.3 Results and discussion $\quad 54$

2.3.3.1 Parameter estimation $\quad 54$

2.3.3.2 Sensitivity analysis $\quad 56$

2.3.3.3 Error caused by steady-state assumption $\quad 57$

$\begin{array}{lll}2.3 .4 & \text { Conclusions } & 58\end{array}$

2.4 Optimal experimental design for enzyme kinetics of benzaldehyde lyase 59

$\begin{array}{lll}2.4 .1 & \text { Introduction } & 59\end{array}$

$\begin{array}{ll}2.4 .2 & \text { Analysis procedure } \\ 2.4 .3 & 60\end{array}$

$\begin{array}{lll}2.4 .3 & \text { Results and discussion } & 60\end{array}$

$\begin{array}{lll}2.4 .4 & \text { Conclusions } & 63\end{array}$

$\begin{array}{lll}2.5 & \text { Dimethylformamide as cosolvent } & 64\end{array}$ 
$\begin{array}{lll}\text { 2.5.1 Introduction } & 64\end{array}$

2.5.2 Materials and methods $\quad 64$

$\begin{array}{lll}2.5 .3 & \text { Results and discussion } & 65\end{array}$

2.5.3.1 Calibration model 65

2.5.3.2 Parameter estimation using optimized experiments 67

$\begin{array}{lll}2.5 .4 & \text { Conclusions } & 70\end{array}$

2.6 Further aspects concerning the kinetic analysis of benzaldehyde lyase $\quad 70$

2.6.1 Influence of temperature, $\mathrm{pH}$ value, ionic strength, and cosolvent $\begin{array}{ll}\text { concentration } & 70\end{array}$

$\begin{array}{ll}\text { 2.6.2 Active site titration for benzaldehyde lyase } & 71\end{array}$

2.7 Investigating the carboligase activity of thiamin diphosphate-dependent enzymes using mechanistic kinetic modeling and NMR analysis 72

$\begin{array}{lll}2.7 .1 & \text { Introduction } & 72\end{array}$

$\begin{array}{lll}\text { 2.7.2 Materials and methods } & 73\end{array}$

$\begin{array}{lll}2.7 .3 & \text { Results and discussion } & 75\end{array}$

$\begin{array}{lll}2.7 .4 & \text { Conclusions } & 81\end{array}$

\section{Mass Transfer Between Two Liquid Phases 82}

3.1 Systematic approach for solvent selection 82

3.2 Materials and methods 86

$\begin{array}{lll}3.2 .1 & \text { COSMO-RS } & 86\end{array}$

$\begin{array}{lll}3.2 .2 & \text { Experimental } & 86\end{array}$

$\begin{array}{lll}3.3 & \text { Model development } & 87\end{array}$

$\begin{array}{llr}3.4 & \text { Results and discussion } & 88\end{array}$

3.4.1 Ab initio solvent screening $\quad 88$

$\begin{array}{lll}3.4 .2 & \text { Parameter estimation } & 89\end{array}$

$\begin{array}{ll}\text { 3.4.3 Evaluation of } a b \text { initio predictions } & 92\end{array}$

$\begin{array}{lll}3.5 & \text { Conclusions } & 93\end{array}$ 
4 Diffusion in Hydrogels

4.1 Propionic acid diffusion in Ca-alginate hydrogel beads 95

$\begin{array}{lll}\text { 4.1.1 Introduction } & 95\end{array}$

4.1.2 Model development 96

$\begin{array}{ll}\text { 4.1.3 A priori simulations to check for discriminability } & 100\end{array}$

$\begin{array}{lll}4.1 .4 & \text { Sensitivity analysis } & 102\end{array}$

$\begin{array}{lll}4.1 .5 & \text { Experimental methods } & 104\end{array}$

4.1.6 Optimal experimental design for model discrimination 108

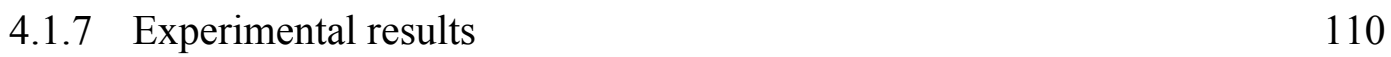

4.1.8 Parameter estimation and model discrimination 111

$\begin{array}{lll}\text { 4.1.8.1 First MEXA cycle } & 111\end{array}$

$\begin{array}{lll}\text { 4.1.8.2 Second MEXA cycle } & 113\end{array}$

$\begin{array}{lll}4.1 .9 & \text { Conclusions } & 116\end{array}$

$\begin{array}{lll}\text { 4.2 Diffusion in } \kappa \text {-carrageenan hydrogel beads } & 117\end{array}$

$\begin{array}{lll}\text { 4.2.1 Introduction } & 117\end{array}$

$\begin{array}{lll}\text { 4.2.2 } & \text { Model development } & 117\end{array}$

$\begin{array}{lll}4.2 .3 & \text { Sensitivity analysis } & 119\end{array}$

$\begin{array}{lll}\text { 4.2.4 Experimental methods } & 121\end{array}$

4.2.5 Experimental results and parameter estimation 122

$\begin{array}{lll}\text { 4.2.6 Conclusions } & 125\end{array}$

$\begin{array}{llr}4.3 & \text { Diffusion in } \kappa \text {-carrageenan hydrogel cylinders } & 125\end{array}$

$\begin{array}{lll}\text { 4.3.1 Introduction } & 125\end{array}$

$\begin{array}{ll}\text { 4.3.2 Materials and methods } & 126\end{array}$

$\begin{array}{lll}\text { 4.3.3 Model development } & 127\end{array}$

$\begin{array}{lll}\text { 4.3.4 Results and discussion } & 128\end{array}$

$\begin{array}{lll}4.3 .5 & \text { Conclusions } & 131\end{array}$

\section{Coupling of Enzyme and Mass Transfer Kinetics 132}

$\begin{array}{lll}\text { 5.1 Biphasic reactive system with two distinct phases } & 132\end{array}$

$\begin{array}{lll}\text { 5.1.1 Introduction } & 132\end{array}$

$\begin{array}{ll}\text { 5.1.2 Model development } & 133\end{array}$

$\begin{array}{lll}\text { 5.1.3 A priori simulations } & 134\end{array}$ 
5.1.4 Sensitivity analysis

5.1.5 Materials and methods

5.1.6 Results and discussion

5.1.7 Conclusions

5.2 Reactive emulsion

5.2.1 Introduction

5.2.2 Materials and methods

5.2.3 Results and discussion

5.2.4 Conclusions

6.1 Introduction

6.2 Model development

6.3 A priori simulations

6.4 Sensitivity analysis

6.5 Materials and methods

6.6 Results and discussion

6.7 Comparison with NMR

6.8 Conclusions

\section{Enzyme Kinetics in a Gel-Stabilized Aqueous-Organic Biphasic System}

$\begin{array}{lll}7.1 & \text { Introduction } & 167\end{array}$

$\begin{array}{lll}\text { 7.2 } & \text { Model development } & 168\end{array}$

$\begin{array}{llr}7.3 & \text { A priori simulations } & 169\end{array}$

$\begin{array}{lll}7.4 & \text { Sensitivity analysis } & 172\end{array}$ 
$\begin{array}{lll}7.5 & \text { Materials and methods } & 175\end{array}$

$\begin{array}{lll}\text { 7.6 } & \text { Results and discussion } & 177\end{array}$

$\begin{array}{lll}\text { 7.7 Comparison of parameter estimates } & 181\end{array}$

$\begin{array}{lll}7.8 & \text { Conclusions } & 184\end{array}$

8 Conclusions and Outlook 186

$\begin{array}{ll}\text { Bibliography } & \text { XXXV }\end{array}$

Lebenslauf $\quad$ LX 


\section{Nomenclature}

\section{Abbreviations}

a.u. arbitrary units

ANB

5-amino-2-nitrobenzoic acid

BAL

benzaldehyde lyase

BFD

benzoylformate decarboxylase

CLSM

confocal laser scanning microscopy

COSMO-RS

Conductor-like Screening Model for Realistic Solvation

DMBA

3,5-dimethoxy-benzaldehyde

DMF

dimethylformamide

DMSO

dimethylsulfoxide

EC

enzyme commission

FDA

Food and Drug Administration

FTIR

Fourier transformed infrared

GC

gas chromatography

gPROMS

general process modeling system

$\mathrm{Ha}$

Hatta number

HBz-ThDP

hydroxybenzyl thiamin diphosphate

$\mathrm{HPa}$

propionic acid

HPLC

high performance liquid chromatography

ITMC

Institute for Technical and Macromolecular Chemistry

LLE liquid-liquid equilibria

LSM laser scanning microscopy

MEXA

model-based experimental analysis 


$\begin{array}{ll}\text { MIBK } & \text { methyl-iso-butyl-ketone } \\ \text { MTBE } & \text { methyl-tert-butyl-ether } \\ \text { NIPAB } & \text { 6-nitro-3-phenylacetamido benzoic acid } \\ \text { NMR } & \text { nuclear magnetic resonance } \\ \text { OED } & \text { optimal experimental design } \\ \text { PA } & \text { penicillin amidase } \\ \text { Pa } & \text { propionate } \\ \text { PAA } & \text { phenylacetic acid } \\ \text { PDC } & \text { pyruvate decarboxylase } \\ \text { PMT } & \text { photomultiplier tube } \\ \text { RSCB } & \text { Research Collaboratory for Structural Bioinformatics } \\ \text { Sh } & \text { Sherwood number } \\ \text { ThDP } & \text { thiamin diphosphate } \\ \text { TMB } & \text { Tris(hydroxymethyl)-aminomethan } \\ \text { Tris } & \end{array}$

\section{Roman Symbols}

$\begin{array}{lll}a & \text { fluorescence calibration parameter } & \text { [a.u. } \mathrm{mM}^{-3} \text { ] } \\ a & \text { CLSM calibration parameter } & \text { [a.u. } \mathrm{mM}^{-1} \text { ] } \\ a & \text { fluorescence proportion of acidic component } & \text { [a.u.] } \\ A & \text { mass transfer area } & {\left[\mathrm{m}^{2}\right]} \\ b & \text { background extinction } & {[-]} \\ b & \text { fluorescence calibration parameter } & \text { [a.u. } \mathrm{mM}^{-2} \text { ] } \\ b & \text { fluorescence proportion of basic component } & \text { [a.u.] } \\ C & \text { variance covariance matrix } & \end{array}$


c

c

$d$

$d$

$d$

D

e

Ext

f

F

$g$

I

I

$j$

$J$

$k$

$k$

$k_{B}$

$k_{\text {catf }}$

$k_{\text {catr }}$

$k_{i}$

$K_{e q}$

$K_{i A}$

$K_{i B}$

$K_{i P}$ fluorescence calibration parameter

concentration

pathlength

fluorescence calibration parameter

alginate distribution parameter

diffusion coefficient

fluorescence calibration parameter

extinction

fluorescence calibration parameter

Faraday constant

fluorescence calibration parameter

intensity

ionic strength

molar diffusive flux

Jacobian matrix

micro-reaction rate constant

matrix interaction parameter

Boltzmann constant

maximum turnover number

maximum turnover number for reverse reaction

overall mass transfer coefficient

equilibrium constant

inhibition constant for first binding substrate

inhibition constant for second binding substrate

inhibition constant for first product [a.u. $\mathrm{mM}^{-1}$ ]

$[\mathrm{mM}]$

[m]

[a.u. $\mathrm{mM}^{-1}$ ]

$\left[\mathrm{m}^{-2}\right]$

$\left[\mathrm{m}^{2} \mathrm{~s}^{-1}\right]$

[a.u. $\mathrm{mM}^{-3}$ ]

[-]

[a.u. $\mathrm{mM}^{-2}$ ]

[A s mol-1]

[a.u. $\mathrm{mM}^{-1}$ ]

[a.u.]

[mM]

$\left[\mathrm{mol} \mathrm{m} \mathrm{m}^{-2} \mathrm{~s}^{-1}\right]$

$\left[\mathrm{s}^{-1}\right],\left[\mathrm{mM}^{-1} \mathrm{~s}^{-1}\right]$

[-]

$\left[\mathrm{J} \mathrm{K}^{-1}\right]$

$\left[\mathrm{s}^{-1}\right]$

$\left[\mathrm{s}^{-1}\right]$

$\left[\mathrm{m} \mathrm{s}^{-1}\right]$

$\left[\mathrm{mM}^{-1}\right]$

[mM]

[mM]

[mM] 


\begin{tabular}{|c|c|c|}
\hline$K_{i Q}$ & inhibition constant for second product & {$[\mathrm{mM}]$} \\
\hline$K_{m A}$ & Michaelis constant for first binding substrate & {$[\mathrm{mM}]$} \\
\hline$K_{m B}$ & Michaelis constant for second binding substrate & {$[\mathrm{mM}]$} \\
\hline$K_{m P}$ & Michaelis constant for product & {$[\mathrm{mM}]$} \\
\hline$K_{W}$ & ion product of water & {$\left[\mathrm{mM}^{2}\right]$} \\
\hline$n$ & amount of substance & {$[\mathrm{mol}]$} \\
\hline$n$ & rotating frequency & {$[\mathrm{rpm}]$} \\
\hline$n$ & number of experiments & {$[-]$} \\
\hline$\dot{n}_{i, M T}$ & mass transfer flux & {$\left[\mathrm{mol} \mathrm{s}^{-1}\right]$} \\
\hline$\dot{n}_{i, R}$ & reaction flux & {$\left[\mathrm{mol} \mathrm{s}^{-1}\right]$} \\
\hline$P_{i}$ & partition coefficient & {$[-]$} \\
\hline$q$ & fluorescence calibration parameter & {$[-]$} \\
\hline$r$ & radial position & {$[\mathrm{m}]$} \\
\hline$r$ & molecule radius & {$[\mathrm{m}]$} \\
\hline$R$ & ratio of partition coefficients ( $\mathrm{R}$-factor) & {$[-]$} \\
\hline$R$ & bead radius & {$[\mathrm{m}]$} \\
\hline$R_{m}$ & molecular gas constant & {$\left[\mathrm{J} \mathrm{mol}^{-1} \mathrm{~K}^{-1}\right]$} \\
\hline$s$ & background fluorescence & [a.u.] \\
\hline$S$ & normalized sensitivity & {$[-]$} \\
\hline$t$ & time & {$[\mathrm{s}]$} \\
\hline$t_{\text {lag }}$ & lag-time & {$[\mathrm{s}]$} \\
\hline$T$ & temperature & {$[\mathrm{K}]$} \\
\hline$v$ & reaction rate & {$\left[\mathrm{mM} \mathrm{s}^{-1}\right]$} \\
\hline$V$ & volume & {$\left[\mathrm{m}^{3}\right]$} \\
\hline$w$ & mass fraction & {$[-]$} \\
\hline
\end{tabular}




$\begin{array}{llc}Y & \text { yield } & {[-]} \\ z & \text { charge } & {[-]} \\ z & \text { axial position } & {[\mathrm{m}]}\end{array}$

\section{Greek symbols}

$\delta$

$\varepsilon$

$\varphi$

$\eta$

$\eta$

$\lambda$

$\mu$

$v$

$\sigma$

$\theta$

$\tau$

$\Phi$

$\Psi$

\section{Superscripts}
$+$
positively charged
negatively charged
$N$
Fick's law
Nernst-Planck law

F

objective function
$-$

$[-]$

m]

[m]

$\left[\mathrm{mM}^{-1} \mathrm{~cm}^{-1}\right]$

$\left[\mathrm{kg} \mathrm{m}^{-1} \mathrm{~s}^{-1}\right]$

$[-]$

$[-]$

$\left[\mathrm{J} \mathrm{mol}^{-1}\right]$

$[-]$

$\left[\mathrm{s}^{-1}\right],\left[\mathrm{mM}^{-1}\right],[\mathrm{mM}]$

[s]

$\left[\mathrm{J} \mathrm{A}^{-1} \mathrm{~s}^{-1}\right]$ 


\section{Subscripts}

\begin{tabular}{|c|c|}
\hline 0 & initial \\
\hline 0 & in free solution \\
\hline 1 & phase 1 \\
\hline 1 & first micro-reaction step \\
\hline-1 & first micro-reaction step (reverse) \\
\hline 2 & phase 2 \\
\hline 2 & second micro-reaction step \\
\hline-2 & second micro-reaction step (reverse) \\
\hline 3 & third micro-reaction step \\
\hline-3 & third micro-reaction step (reverse) \\
\hline$A$ & substrate, first binding substrate \\
\hline$A$ & acidic component \\
\hline alg & alginate \\
\hline$a q$ & aqueous phase \\
\hline$B$ & second binding substrate \\
\hline$B$ & basic component \\
\hline bead & hydrogel bead \\
\hline bulk & bulk phase \\
\hline cylinder & hydrogel cylinder \\
\hline E & enzyme \\
\hline$E A$ & enzyme substrate complex \\
\hline$E A A$ & ternary complex \\
\hline$E A B$ & ternary complex \\
\hline eff & effective \\
\hline
\end{tabular}




$\begin{array}{ll}\text { eq } & \text { equilibrium } \\ \text { exp } & \text { experiment } \\ \text { gel } & \text { hydrogel } \\ \text { min } & \text { minimal } \\ \text { max } & \text { maximal } \\ \text { org } & \text { organic phase } \\ P & \text { product } \\ \text { ref } & \text { reference } \\ \text { smooth } & \text { smoothed } \\ \text { tot } & \text { total }\end{array}$




\section{Figures}

Figure 1-1: Gel-stabilized aqueous-organic biphasic reaction system.

Figure 1-2: Work process of the model-based experimental analysis (MEXA) approach (adapted from Marquardt (2005)).

Figure 1-3: Separation and coupling of occurring phenomena. Each number indicates the chapter of this thesis, in which the respective system is analyzed.

Figure 2-1: Progress curves with different initial concentrations of NIPAB (experimental: a; in silico: b) and PAA (experimental: $\mathrm{c}$; in silico: d). The letters in the legend refer to the experiments listed in Table 2-1.

Figure 2-2: Progress curve of in silico experiment B with the fitted smoothing spline (a); calculated reaction rate using finite differences (b) or differentiated smoothing splines (c). The NIPAB concentration was calculated using the raw data (b), or the smoothed data (c).

Figure 2-3: Deviations of the algebraic parameter estimates for $k_{c a t}, K_{i Q}$, and $K_{m A}$ from the preset values for the in silico progress curves. Finite differences are compared with differentiated smoothing splines using MS-Excel or Origin as computer program.

Figure 2-4: $\quad$ Parameter estimates for $k_{c a t}, K_{i Q}$, and $K_{m A}$ using experimental or in silico progress curves. The dotted lines indicate the preset values for the kinetic parameters. For each parameter and amount of data points the maximum difference among the computer programs is specified. Standard deviations are provided where applicable.

Figure 2-5: Confidence ellipsoids of the kinetic parameters obtained from gPROMS.

Figure 2-6: Applied procedure for deriving the kinetic model.

Figure 2-7: Synthesis reaction of 3,5-dimethoxy-benzaldehyde (DMBA) to $(R)$ 3,3',5,5'-tetramethoxy-benzoin (TMB) (A). Reaction mechanism of BAL for the TMB synthesis adapted from Demir et al. (2001b) (B). Reaction mechanism of a bi-uni-reaction according to Cleland (1963) (C). 
Figure 2-8: Comparison of the fitted mechanistic kinetic model with the experimental data using experiments A-I (Table 2-4). The residuals are shown as small inlets.

Figure 2-9: $\quad 95 \%$ confidence ellipsoids for $K_{\text {eq }} / k_{\text {catf }}$ (top) and $K_{m P} / K_{i A}$ (bottom).

Figure 2-10: $95 \%$ confidence ellipsoid for $K_{m P} / K_{i A}$ after performing in silico experiments.

Figure 2-11: Normalized sensitivities of the model parameters of the mechanistic model regarding the substrate concentration using the initial conditions of experiment A (Table 2-4).

Figure 2-12: Comparison of the fitted simplified kinetic model with the experimental data using experiments A-I (Table 2-4). The residuals are shown as small inlets.

Figure 2-13: Visualization of micro-reaction steps in the BAL mechanism using Cleland's notation (1963).

Figure 2-14: Normalized sensitivities of the model parameters of the simplified kinetic model regarding the substrate concentration.

Figure 2-15: Normalized sensitivities of the micro-reaction rate constants using the micro-kinetic model with both the steady-state and the mechanistic assumption. The initial conditions of experiment A (Table 2-4) were used.

Figure 2-16: Simulated trajectories of substrate and product concentration trajectories with steady-state assumption and without (left). Concentration trajectories of the enzyme intermediates without steady-state assumption (right). The total enzyme concentration is $0.125 \mu \mathrm{M}$, the initial concentrations of substrate and product $3 \mathrm{mM}$ and $0 \mathrm{mM}$, respectively. 
Figure 2-17: Plot of the reaction rate over the substrate and product concentration as contour plot. The reaction rate was calculated using the simplified kinetic model (Section 2.2.3.3) and the parameter values listed in Table 2-12. The color bar indicates reaction rates in $\mathrm{mM} \mathrm{s}^{-1}$. A reaction rate of $0 \mathrm{mM} \mathrm{s}^{-1}$ indicates thermodynamic equilibrium (dashed line). The optimal experiments are visualized by arrows. The experiments R-Y (Table 2-16) were designed as described in Section 2.2.3.2.

Figure 2-18: Influences of the number of experiments (A), the standard deviation of the experimental data (B), and the number of data points per experiment (C) on the precision of the obtained parameter estimates using the simplified kinetic model for BAL. The CPU time needed for OED is plotted versus the number of data points (D).

Figure 2-19: Dependence of fluorescence intensity from substrate and product concentration in the presence of DMF.

Figure 2-20: Comparison of the fitted simplified kinetic model with the experimental data using experiments A-H (Table 2-22). The model was simultaneously fitted to extinction (red) and fluorescence (blue) intensities.

Figure 2-21: 4-Bromomethyl-benzaldehyde - a possible suicide substrate for BAL.

Figure 2-22: Synthesis of two benzaldehyde molecules to $(R)$-benzoin using either BAL or BFD H281A.

Figure 2-23: Selection of progress curves fitted by the simplified kinetic model. For each tested cosolvent the measured concentrations for benzaldehyde (left axis) and benzoin (right axis) are plotted over time.

Figure 2-24: Initial reaction rates of BAL (left) and BFD $H 281 A$ (right) in presence of different cosolvents.

Figure 2-25: Calculated micro-reaction rate constants using the simplified kinetic model. 
Figure 2-26: Crystal structure of BAL (left) and BFD H281A (right). The active sites are enlarged. The pictures are adapted from the RCSB Protein Data Bank entries 2ag0 (BAL) and 1mcz (BFD H281A) which were generated by Dr. Michael Knoll, Institute of Technical Biochemistry, University of Stuttgart.

Figure 2-27: Scheme for the cross section of the active sites for BAL (top) and BFD H281A (bottom) adapted from Knoll et al. (2006).

Figure 2-28: Distribution of reaction intermediates from steady-state intermediate analysis by ${ }^{1} \mathrm{H}$ NMR. The $2^{2}-\mathrm{CH}_{3}$ and the $4-\mathrm{CH}_{3}$ fingerprint region is shown for BAL (left) and BFD H281A (right) in aqueous buffer.

Figure 3-1: Maximum extractable yield for the symmetric carboligation in a biphasic reaction system dependent on the partition coefficients $\left(V_{o r g} / V_{a q}=1\right.$; $\left.c_{A, 0}=10 \mathrm{mM} ; K_{e q}=1.464\right)$.

Figure 3-2: The proposed systematic approach for solvent selection.

Figure 3-3: Fit of the kinetic model to five mass transfer experiments using the substrate DMBA and the organic solvent MIBK. The upper three experiments were started with the addition of $10 \mathrm{mM}$ DMBA to the aqueous phase, the lower two with $5 \mathrm{mM}$ DMBA $\left(25^{\circ} \mathrm{C}, 25 \%(\mathrm{v} / \mathrm{v})\right.$ DMF).

Figure 3-4: Fit of the kinetic model to five mass transfer experiments using the product TMB and the organic solvent MIBK. The upper three experiments were started with the addition of $5 \mathrm{mM}$ TMB to the aqueous phase, the lower two with $2.5 \mathrm{mM}$ TMB $\left(25^{\circ} \mathrm{C}, 25 \%(\mathrm{v} / \mathrm{v}) \mathrm{DMF}\right)$.

Figure 3-5: Influence of the temperature on the partition coefficients and on $R$ using $n$-hexane as organic solvent $(25 \%(\mathrm{v} / \mathrm{v}) \mathrm{DMF})$. The partition coefficients were determined after 24 hours using HPLC.

Figure 3-6: Influence of the volume fraction of the cosolvent DMF on partition coefficients and on $R$ using $n$-hexane as organic solvent $\left(25^{\circ} \mathrm{C}\right)$. The partition coefficients were determined after 24 hours using HPLC.

Figure 3-7: Comparison of ab initio predictions of COSMO-RS with the values obtained after fitting the kinetic model to experimental data. 
Figure 4-1: $\quad$ Simulated $\mathrm{pH}$ value over time in the hydrogel particle core after $30 \mathrm{~s}$ and $50 \mathrm{~s}$ assuming different (linear, exponential, hyperbolic) dependence of the diffusion coefficient on the local alginate density (Eqs. 4-15-17). Nernst-Planck diffusion is chosen as diffusion model. Simulation parameters: $R=1.15 \mathrm{~mm}, \mathrm{p} H_{\text {bead }, 0}=6.5, c_{P a, t o t, 0}=100 \mathrm{mM}$, $\mathrm{w}_{\mathrm{alg}}=3.3 \%(\mathrm{w} / \mathrm{w}), d=6000 \mathrm{~g} \mathrm{~g}^{-1} \mathrm{~m}^{-2}, k=6.60 \mathrm{~g} \mathrm{~g}^{-1}$.

Figure 4-2: $\quad$ Sensitivity analysis for the parameters bead radius $R$, matrix interaction parameter $k$, and bulk volume $V_{\text {bulk }}$ in the bulk phase (left) and in the bead center (right). For bulk measurements the sensitivity refers to the propionic acid concentration, for the bead measurements to the $\mathrm{pH}$ value $\left(R=1.15 \mathrm{~mm}, \quad \mathrm{p} H_{\text {bead }, 0}=6.5, \quad c_{P a, t o t, 0}=100 \mathrm{mM}, \quad V_{\text {bulk }}=300 \mu \mathrm{L}\right.$, $\left.k=6.60 \mathrm{~g} \mathrm{~g}^{-1}\right)$.

Figure 4-3: Device for the determination of bead roundness. Digital pictures were taken from an illuminated hydrogel bead and its four mirror images and subsequently analyzed quantitatively.

Figure 4-4: Calibration of the $\mathrm{pH}$ value vs. the proportion of acidic and basic dissociation forms $a$ and $b$ of resorufin as determined from biexponential decay curves. The error bars indicate the standard deviation based on 10 repetitions.

Figure 4-5: Simulation of the development of $\mathrm{pH}$ value over time at two different positions in a hydrogel bead: center of the bead $(\mathrm{r} / R=0)$; middle between the center and the surface of the bead $(\mathrm{r} / R=0.5)$. Fickian and Nernst-Planck diffusion are shown for an initial bulk phase concentration of $100 \mathrm{mM}$ propionic acid into a Ca-alginate hydrogel bead of $R=1.25 \mathrm{~mm}$ radius with an initial $\mathrm{pH}$ value of 6.8 .

Figure 4-6: Experimental and estimated $\mathrm{pH}$ values in the bead center for the diffusion of propionic acid into $\mathrm{Ca}$-alginate hydrogel beads. The initial model as described in Section 4.1.2 was used $(R=1.025 \mathrm{~mm}$, Estimated parameters: $p H_{0}=6.79, c_{P a, t o t, 0}=95 \mathrm{mM}$, alginate influence parameter $\left.k_{F}=5.1 \mathrm{~g} \mathrm{~g}^{-1}, k_{N}=6.6 \mathrm{~g} \mathrm{~g}^{-1}\right)$. 
Figure 4-7: Simulation of the chloride concentration in the bead (dimensionless radius $<1$ ) and in the bulk phase (dimensionless radius $>1$ ) using the unrestricted diffusion model and Nernst-Planck law $(R=1.025 \mathrm{~mm}$, $\left.p H_{0}=7.0, c_{P a, t o t, 0}=100 \mathrm{mM}\right)$.

Figure 4-8: $\quad$ Simulated $\mathrm{pH}$ progress in the bead center for Fickian and Nernst-Planck diffusion using the unrestricted model $\left(R=1.005 \mathrm{~mm}, p H_{0}=6.79\right.$, $\left.c_{P a, t o t, 0}=99.1 \mathrm{mM}, k=0.051\right)$.

Figure 4-9: Experimental and estimated $\mathrm{pH}$ values over time for the diffusion of propionic acid into $\mathrm{Ca}$-alginate hydrogel beads using the unrestricted $\operatorname{model}\left(R=1.005 \mathrm{~mm}, p H_{0}=6.79, c_{P a, t o t, 0}=99.1 \mathrm{mM}, k=0.051\right)$.

Figure 4-10: Sensitivity analysis for the diffusion coefficient of DMBA $D_{A}$, the bulk volume $V_{\text {bulk, }}$, and the bead radius $R$ in the bulk phase (left) and in the bead center (right). The sensitivity refers to the DMBA concentration. The sensitivities were normalized as shown in Chapter 4.1.4 $\left(c_{A, b u l k, 0}=50 \mathrm{mM}, D_{A}=5.57 \cdot 10^{-10} \mathrm{~m}^{2} \mathrm{~s}^{-1}, R=1 \mathrm{~mm}, V_{\text {bulk }}=300 \mu \mathrm{L}\right)$.

Figure 4-11: Normalized sensitivity of the diffusion coefficient $D_{A}$ inside the hydrogel bead. The sensitivity refers to the DMBA concentration. The sensitivity was normalized as shown in Chapter $4.1 .4 \quad\left(c_{A, b u l k, 0}=50 \mathrm{mM}\right.$, $\left.D_{A}=5.57 \cdot 10^{-10} \mathrm{~m}^{2} \mathrm{~s}^{-1}, R=1 \mathrm{~mm}, V_{\text {bulk }}=300 \mu \mathrm{L}\right)$.

Figure 4-12: Comparison of the fitted diffusion model with the experimental data for DMBA diffusion. 6 of 27 fits are shown as example. The roundness of each bead is provided and the residuals are shown as small inlets (nominal conditions: $c_{A, b u l k, 0}=25 \mathrm{mM}, R=1.5 \mathrm{~mm}$ ).

Figure 4-13: Comparison of the fitted diffusion model with the experimental data for TMB diffusion. 6 of 27 fits are shown as example. The roundness of each bead is provided and the residuals are shown as small inlets (nominal conditions: $c_{P, b u l k, 0}=1.5-2.5 \mathrm{mM}, R=1.5 \mathrm{~mm}$ ).

Figure 4-14: Illustration of unround beads (exaggerated for visualization).

Figure 4-15: The diffusion cell consisting of a hydrogel phase below a bulk phase. Direction of DMBA diffusion is indicated with the arrow. The coordinate system in each phase is labeled as $z_{b u l k}$ and $z_{c y l i n d e r}$. 
Figure 4-16: Comparison of the fitted diffusion model with the experimental data for DMBA diffusion using ${ }^{1} \mathrm{H}$ NMR. The residuals are shown as small inlets. The optimized experimental conditions listed in Table 4-4 were applied.

Figure 5-1: $\quad 95 \%$ confidence ellipsoids for $k_{A} / k_{\text {catf }}$ for the four investigated test cases.

Case 1: only $c_{A, a q}$ quantifiable; case $2: c_{A, a q}$ and $c_{P, a q}$ quantifiable; case 3 : $c_{A, a q}, \quad c_{P, a q}$, and $c_{A, \text { org }}$ quantifiable; case 4 : all concentrations are quantifiable.

Figure 5-2: Normalized sensitivities of the model parameters regarding $c_{A, \text { org }}$. The initial conditions were $c_{A, \text { org }}=10 \mathrm{mM}, c_{A, a q}=0 \mathrm{mM}, c_{P, a q}=0 \mathrm{mM}$, and $c_{P, \text { org }}=0 \mathrm{mM}$. The enzyme concentration was $c_{E}=3.34 \cdot 10^{-4} \mathrm{mM}$. The sensitivities were calculated via automatic differentiation.

Figure 5-3: Normalized sensitivities of the model parameters regarding $c_{A, \text { org }}$. The initial conditions were $c_{A, \text { org }}=0 \mathrm{mM}, c_{A, a q}=0.96 \mathrm{mM}, c_{P, a q}=2.5 \mathrm{mM}$, and $c_{P, \text { org }}=0 \mathrm{mM}$. The enzyme concentration was $c_{E}=3.38 \cdot 10^{-7} \mathrm{mM}$. The sensitivities were calculated via automatic differentiation.

Figure 5-4: Illustration of the concentration profiles at the interface for a reaction limited regime (left) and for a (partially) mass transfer limited regime (right). $\delta_{\mathrm{i}}$ denotes the film thickness.

Figure 5-5: Newly developed reactor concept which allows measurements in both phases

Figure 5-6: Fit of the kinetic model to experimental data from extinction and fluorescence measurements. The experimental conditions are listed in Table 5-4. (In experiment E the first $120 \mathrm{~s}$ were omitted due to large noise. In experiment I the extinction measurements in the organic phase were defective and could not be used.) 
Figure 5-7: Emulsification experiment using the vertical disc stir bar and two different overhead stirrers. The upper row shows results for a blade stirrer with $3 \mathrm{~mm}$ diameter, the lower row for a blade stirrer with $7 \mathrm{~mm}$ diameter. The shaft length was in each case $25 \mathrm{~mm}$. The rotation frequencies of the overhead stirrers were increased from left to right. The rotation frequency of the vertical stir bar was kept constant at $725 \mathrm{rpm}$. MIBK was used as organic solvent. The aqueous phase was colored using $\mathrm{CuSO}_{4} . V_{\text {org }}=1 \mathrm{~mL}, V_{a q}=2 \mathrm{~mL}$.

Figure 5-8: $\quad$ Mass transfer experiments for DMBA in emulsions. DMBA was added in different concentrations to the organic MIBK phase $\left(V_{\text {org }}=1 \mathrm{~mL}\right.$, $\left.V_{a q}=2 \mathrm{~mL} ; n_{\text {overhead stirrer }}=2400 \mathrm{rpm}, n_{\text {vertical disc stir bar }}=725 \mathrm{rpm}\right)$.

Figure 5-9: Calculated time course of the Hatta number $\mathrm{Ha}$ in the emulsified system assuming that the nominal values for the enzyme kinetic parameters (Table 5-2) are valid $\left(V_{\text {org }}=1 \mathrm{~mL}, V_{a q}=2 \mathrm{~mL} ; c_{E}=1.02 \cdot 10^{-4} \mathrm{mM}\right.$; $\left.c_{A, \text { org }, 0}=5 \mathrm{mM} ; c_{A, a q, 0}=c_{P, \text { org }, 0}=c_{P, a q, 0}=0\right)$.

Figure 5-10: Simulated concentration profiles for the reactive emulsion assuming that the nominal values for the enzyme kinetic parameters (Table 5-2) are valid $\left(V_{\text {org }}=1 \mathrm{~mL}, \quad V_{a q}=2 \mathrm{~mL} ; \quad c_{E}=1.02 \cdot 10^{-4} \mathrm{mM} ; \quad c_{A, \text { org }, 0}=5 \mathrm{mM}\right.$; $\left.c_{A, a q, 0}=c_{P, \text { org }, 0}=c_{P, a q, 0}=0\right)$.

Figure 5-11: Aggregates formed after emulsification of the biphasic system in presence of enzyme $\left(V_{\text {org }}=1 \mathrm{~mL}, \quad V_{a q}=2 \mathrm{~mL} ; \quad c_{E}=5.75 \cdot 10^{-4}\right.$; $\left.c_{A, \text { org }, 0}=25 \mathrm{mM} ; c_{A, a q, 0}=c_{P, \text { org }, 0}=c_{P, a q, 0}=0\right)$.

Figure 6-1: A priori simulations of the DMBA concentration in the bead center. Three test cases are investigated: Case 1: Addition of $20 \mathrm{mM}$ DMBA to the bulk phase (dotted line). Case 2: Addition of $1000 \mathrm{mM} \mathrm{DMBA}$ to the bulk phase (solid line). Case 3: Addition of $20 \mathrm{mM}$ DMBA to the hydrogel bead (dashed line) (all other concentrations are zero, $R=1.5 \mathrm{~mm})$. 
Figure 6-2: $\quad$ Sensitivity analysis for the diffusion coefficient of DMBA $D_{A, \text { eff }}$, the bulk volume $V_{b u l k}$, the bead radius $R$, and the partition coefficient of DMBA $P_{A}$ in the bulk phase (left) and in the bead center (right). The sensitivity refers to the DMBA concentration. The nominal values of the parameters are listed in Table $6-1\left(c_{A, \text { bead }, 0}=20 \mathrm{mM}, R=1.5 \mathrm{~mm}, V_{\text {bulk }}=250 \mu \mathrm{L}\right)$.

Figure 6-3: Comparison of the fitted kinetic model for diffusion and mass transfer with the experimental data for DMBA diffusion. 4 of 12 fits are shown as example. The residuals are shown as small inlets (nominal conditions: $\left.c_{A, g e l, 0}=15-20 \mathrm{mM}, R=1.5 \mathrm{~mm}\right)$.

Figure 6-4: Comparison of the fitted kinetic model for diffusion and mass transfer with the experimental data for TMB diffusion. 4 of 10 fits are shown as example. The residuals are shown as small inlets (nominal conditions: $\left.c_{P, g e l, 0}=2-3 \mathrm{mM}, R=1.5 \mathrm{~mm}\right)$.

Figure 6-5: Comparison of the fitted kinetic model with the experimental data for DMBA diffusion at different positions in the hydrogel cylinder using $n$-hexane as organic bulk phase. The experimental data were obtained by ${ }^{1} \mathrm{H}-\mathrm{NMR}$. The residuals are shown as small inlets $\left(c_{A, b u l k, 0}=25 \mathrm{mM}\right.$, $\left.L_{\text {cylinder }}=0.056 \mathrm{~m}, L_{\text {bulk }}=0.025 \mathrm{~m}\right)$.

Figure 7-1: A priori simulations of the substrate concentration in the hydrogel bead using different enzyme concentrations (top: $3.34 \cdot 10^{-2} \mathrm{mM}$; middle: $3.34 \cdot 10^{-4} \mathrm{mM}$; $\quad$ bottom: $\left.\quad 3.34 \cdot 10^{-6} \mathrm{mM}\right) \quad\left(c_{A, b u l k, 0}=400 \mathrm{mM}\right.$, $\left.V_{\text {bulk }}=300 \mu \mathrm{L}, R=1.5 \mathrm{~mm}\right)$.

Figure 7-2: Normalized sensitivities of the model parameters regarding the DMBA concentration in the bulk phase (left) and in the bead center (right). The nominal parameter values listed in Table $7-1$ were used. Based on the previous results it was assumed that the mass transfer at the interface is not rate-limiting $\quad\left(c_{E}=3.34 \cdot 10^{-4} \mathrm{mM}, \quad c_{D M B A, b u l k, 0}=400 \mathrm{mM}\right.$, $\left.V_{\text {bulk }}=300 \mu \mathrm{L}, R=1.5 \mathrm{~mm}\right)$.

Figure 7-3: Normalized sensitivity regarding the DMBA concentration of the parameter $K_{m A}$ as a function of time and radius. The nominal parameter values listed in Table $7-1$ were used $\left(c_{E}=6.69 \cdot 10^{-4} \mathrm{mM}\right.$, $\left.c_{A, b u l k, 0}=400 \mathrm{mM}, V_{\text {bulk }}=300 \mu \mathrm{L}, R=1.5 \mathrm{~mm}\right)$. 
Figure 7-4: Fit of the kinetic model to experimental data at 8 different radial positions. 4 of 12 experiments are shown as example. The residuals are shown as small inlets. The experimental conditions are: $c_{A, \text { bulk }, 0}=400 \mathrm{mM}, \quad c_{P, \text { bulk }, 0}=0 \mathrm{mM}, \quad c_{A, \text { bead }, 0}=0 \mathrm{mM}, \quad c_{P, \text { bulk, }}=0 \mathrm{mM}$, $c_{E}=3.34 \cdot 10^{-4} \mathrm{mM}, V_{\text {bulk }}=300 \mu \mathrm{L}, R=1.5 \mathrm{~mm}$. 


\section{Tables}

Table 2-1: Initial conditions for each experiment. The concentrations of ANB and active enzyme were always $0 \mathrm{mM}$ and $2.86 \cdot 10^{-4} \mathrm{mM}$, respectively.

Table 2-2: $\quad$ Preset values of the kinetic parameters for the in silico experiments.

Table 2-3: Deviations from preset values using the computer programs (averaged over $k_{c a t}, K_{i Q}$ and $\left.K_{m A}\right)$.

Table 2-4: $\quad$ Initial experimental conditions.

Table 2-5: Definitions of the model parameters for the mechanistic kinetic model.

Table 2-6: Parameter estimates and standard deviations using the derived mechanistic kinetic model.

Table 2-7: Correlation matrix of the estimated parameters using the derived mechanistic kinetic model.

Table 2-8: Optimal experimental design for in silico experiments using the mechanistic kinetic model. For the optimal experimental design the degrees of freedom were the initial substrate concentration $c_{A 0}$ (limits: 0$5 \mathrm{mM}$ ), the initial product concentration $c_{P 0}$ (limits: $0-5 \mathrm{mM}$ ) and the measurement duration $t$ (limits: 50-640 s). The enzyme concentration was held constant to $1.25 \cdot 10^{-4} \mathrm{mM}$.

Table 2-9: $\quad$ Parameter estimates and standard deviations after performing optimal in silico experiments using the mechanistic kinetic model.

Table 2-10: Correlation matrix of the estimated parameters after performing optimal in silico experiments using the mechanistic kinetic model.

Table 2-11: Definitions of the model parameters for the simplified kinetic model.

Table 2-12: $\quad$ Parameter estimates and standard deviations using the simplified kinetic model.

Table 2-13: Correlation matrix of the estimated parameters using the simplified kinetic model. 
Table 2-14: Variance-covariance matrix of the estimated parameters using the simplified kinetic model.

Table 2-15: Calculated micro-reaction rate constants.

Table 2-16: Optimal design for in silico experiments using the simplified kinetic model (R-Y). For the optimal experimental design the degrees of freedom were the initial substrate concentration $c_{A 0}$ (limits: $0-5 \mathrm{mM}$ ), the initial product concentration $c_{P 0}$ (limits: $0-5 \mathrm{mM}$ ) and the measurement duration $t$ (limits: 50-640 s). The enzyme concentration was held constant at $1.25 \cdot 10^{-4} \mathrm{mM}$.

Table 2-17: Parameter estimates and standard deviations after performing optimal in silico experiments using the simplified kinetic model.

Table 2-18: Correlation matrix of the estimated parameters after performing optimal in silico experiments using the simplified kinetic model.

Table 2-19: Estimated values of the micro-reaction rate constants and their standard deviations. The estimations were performed either with or without the steady-state assumption and either with or without the mechanistic assumption introduced in Section 2.2.3.3.

Table 2-20: Correlation matrix of estimated micro-reaction rate constants using the steady-state and the mechanistic assumption.

Table 2-21: Parameter estimates of the fluorescence calibration model (excitation wavelength: $360 \mathrm{~nm}$; emission wavelength: $470 \mathrm{~nm}$ )

Table 2-22: Optimized experiments: The degrees of freedom for the OED were the initial substrate concentration $c_{A 0}$ (limits: $0-10 \mathrm{mM}$ ), the initial product concentration $c_{P 0}$ (limits: $0-10 \mathrm{mM}$ ). the enzyme concentration $c_{E}$ (limits: $\left.10^{-5}-5 \cdot 10^{-4} \mathrm{mM}\right)$, and the experiment duration $t_{\exp }(50-10000 \mathrm{~s})$.

Table 2-23: Parameter estimates and standard deviations using the derived mechanistic kinetic model.

Table 2-24: Correlation matrix of the estimated parameters using the derived mechanistic kinetic model. 
Table 2-25: Parameter estimates and standard deviations using the simplified kinetic model.

Table 2-26: Correlation matrix of the estimated parameters using the simplified kinetic model.

Table 2-27: Estimated micro-reaction rate constants with their standard deviations.

Table 2-28: Estimated independent parameters and their standard deviations for the formation of benzoin from benzaldehyde in $50 \mathrm{mM} \mathrm{K \textrm {K } _ { 2 }} \mathrm{PO}_{4} / \mathrm{K}_{2} \mathrm{HPO}_{4}$ buffer with and without cosolvents. $K_{e q}$ was determined separately to $3.02 \pm 0.13 \mathrm{mM}^{-1}$.

Table 3-1: List of solvents showing a miscibility gap in the COSMOtherm calculations with water at $25^{\circ} \mathrm{C}$, ranked according to their $R$ values.

Table 3-2: Estimated parameters for the kinetic mass transfer model $\left(25^{\circ} \mathrm{C}\right.$, $25 \%(\mathrm{v} / \mathrm{v}) \mathrm{DMF})$.

Table 4-1: $\quad$ Model parameters describing the diffusion of propionic acid into Caalginate hydrogel beads (Heinemann, 2003).

Table 4-2: Degrees of freedom and results for the optimal experimental design. For the optimization Eq. 4-21 and the optimal experimental design facilities of gPROMS (version 3.0.2, Process System Enterprise Ltd., London, UK) were used.

Table 4-3: Estimated effective diffusion coefficients of DMBA and TMB in $\kappa$-carrageenan hydrogel beads.

Table 4-4: Optimal experimental design for a diffusion experiment in hydrogel cylinders. Degrees of freedom were the initial DMBA concentration in the bulk phase $c_{A, b u l k, 0}(0-25 \mathrm{mM})$, the length of bulk phase $L_{b u l k}(0$ $0.15 \mathrm{~m})$, and the duration $t_{\exp }(1-20 \mathrm{~h})$. The length of the cylinder $L_{c y l i n d e r}$ was fixed to $0.05 \mathrm{~m}$.

Table 4-5: Estimated diffusion coefficients of DMBA in the $\mathrm{D}_{2} \mathrm{O}$ bulk phase and in $\kappa$-carrageenan hydrogel.

Table 5-1: $\quad$ Overview over in silico experiments. The enzyme concentration $c_{E}$ is in each case $1.67 \cdot 10^{-5} \mathrm{mM}$ and the experiment duration $t$ is $10,000 \mathrm{~s}$. 
Table 5-2: Nominal values for the model parameters (determined in Chapters 2 and $3)$.

Table 5-3: Calibration parameter for the fluorescence measurements in the aqueous phase of the biphasic system (excitation wavelength: $360 \mathrm{~nm}$; emission wavelength: $470 \mathrm{~nm})$.

Table 5-4: Optimized experiments for the aqueous-organic biphasic reaction system. The degrees of freedom were $c_{A, a q}$ (limits: 0-5 mM), $c_{A, \text { org }}$ (limits: 0$25 \mathrm{mM}), c_{P, a q}$ (limits: 0-2.5 mM), $c_{P, \text { org }}$ (limits: 0-10 mM), $c_{E}$ (limits: $10^{-}$ ${ }^{8}-10^{-5} \mathrm{mM}$ ), and $t$ (limits: 600-14,400 s). Additional constraints were $c_{A, \text { org }}+c_{P, \text { org }}<25 \mathrm{mM}$ and $\mathrm{Ha}<0.3$.

Table 5-5: Comparison of the nominal parameter values with the estimates using the biphasic reactive system. The mass transfer coefficients were not identifiable due to reaction limitation.

Table 5-6: Correlation matrix of the estimated parameters using the biphasic reactive system.

Table 5-7: Obtained partition and mass transfer coefficient for the substrate in the emulsion.

Table 6-1: Nominal values for the model parameters. The values were determined in Chapters 3 and 4. The partition coefficients refer to MIBK as organic solvent.

Table 6-2: Estimated effective diffusion coefficients of DMBA and TMB in $\kappa$-carrageenan hydrogel beads using MIBK as bulk phase.

Table 6-3: Estimated diffusion coefficients of DMBA in the $n$-hexane bulk phase and in the $\kappa$-carrageenan hydrogel cylinders.

Table 6-4: Comparison of the estimated diffusion coefficients for DMBA in $\kappa$-carrageenan hydrogel (all values are in $\mathrm{m}^{2} \mathrm{~s}^{-1}$ ).

Table 7-1: Nominal values for the model parameters previously determined in Chapters 2, 3 and 4.

Table 7-2: $\quad$ Obtained parameter estimates and their standard deviations.

Table 7-3: Correlation matrix of the parameter estimates. 
Table 7-4: Comparison of the parameter estimates and their standard deviations for the enzyme reaction. The values for the individual system were determined in Chapter 2.5, those for the coupling of enzyme reaction and mass transfer in Chapter 5.1.

Table 7-5: Comparison of the parameter estimates and their standard deviations for the mass transfer. The values for the individual system were determined in Chapter 3, those for the coupling of enzyme reaction and mass transfer in Chapter 5.1. Due to different geometries the values of the mass transfer coefficients from the individual system cannot be compared to those using hydrogel beads.

Table 7-6: Comparison of the parameter estimates and their standard deviations for the effective diffusion coefficients. The values for the individual system were determined in Chapter 4.2, those for the coupling of diffusion and mass transfer in Chapter 6. 


\section{Introduction}

\subsection{Biocatalytic production of hydrophobic fine chemicals}

Hydrophobic fine chemicals are highly functional molecules with often intricate stereochemistry (Blaser, 2003). There is a large demand for hydrophobic fine chemicals especially in the pharmaceutical, agrochemical and food industry, where they are used as building blocks and performance molecules (Schulze and Wubbolts, 1999; Straathof et al., 2002). For these applications high purities and large quantities are required. Initially the chemical industry applied established chemical synthesis routes for their production, but is turning more and more to biocatalytic processes (Balkenhohl et al., 1997; Breuer et al., 2004; Schmid et al., 2002; Sheldon, 2007; Woodley, 2008). Nowadays, biocatalysis can be considered as standard technology for the production of hydrophobic fine chemicals and is applied by companies such as BASF, DSM, Lonza, Dow Chemical, and DuPont (Schmid et al., 2001; Sheldon, 2005; Straathof et al., 2002). In the future new industrial applications of biocatalysis are expected (Schoemaker et al., 2003; Woodley, 2008).

For these biocatalytic processes both whole cells and enzymes can be used. Enzymes catalyze synthetic reactions with high reaction rates under relatively mild conditions with respect to $\mathrm{pH}$, temperature and pressure. The most important advantages of enzymes are their high specifity and selectivity. Thus, less side products are formed and enantiopure products can be obtained (Leresche and Meyer, 2006; Schulze and Wubbolts, 1999). The production of enantiopure substances is increasingly enforced by the US Food and Drug Administration (FDA) in order to minimize possible side effects. Moreover, this may be economically favorable since less quantities are needed (Breuer et al., 2004). The chemical synthesis of enantiopure substances usually requires blocking and deblocking steps by means of protective groups. Therefore, the number of process steps can be reduced by using enzymes (Schmid et al., 2001; Woodley, 2008). Additionally, enzymatic processes are often more environmentfriendly compared to conventional chemical processes (Schmid et al., 2002; Sheldon, 2007). However, most enzymes show their highest activity in aqueous media, which can be a problem for the production of hydrophobic fine chemicals because of the low solubilities of the substrates in water (Fernandes and Cabral, 2008; Leresche and Meyer, 2006; Villela Filho 
et al., 2003). Furthermore, several products can degrade or racemize in water. The synthesis of some products may be even thermodynamically not feasible in aqueous solution. Examples of such reactions are ester synthesis, polymerization of amino acids or sugars, and dehydration reactions, where water is formed. In aqueous solution the equilibrium of such reactions is strongly shifted towards the substrates (Klibanov, 2001).

\subsection{Enzyme reactions using non-conventional media}

The drawbacks of enzymatic catalysis in homogeneous aqueous solutions can be overcome by using other media than water. Since aqueous media are the natural environment of enzymes, all other media are called "non-conventional". Typical non-conventional media for enzymatic catalysis are gases, supercritical fluids, ionic liquids, and organic solvents and their applications are arising (Cantone et al., 2007; Kragl., 2002; Lamare et al., 2004; Sheldon, 2005). Despite significant advances in engineering of new enzymes with improved tolerance towards non-conventional media, often inactivation of enzymes occurs if water-miscible solvents are used. Therefore, most non-conventional media are applied in multi-phase systems (Cantone et al., 2007; Fernandes and Cabral, 2008; Klibanov, 2001; Schmid et al., 2001).

The potential of aqueous-organic biphasic systems was discovered in the 1970s (e.g. Cremonesi et al., 1973, 1974, 1975; Klibanov et al., 1977). Prior to this, it was believed that organic solvents would generally inhibit enzymes since this effect was observed for watermiscible organic solvents (Butler, 1979). However, if water-immiscible solvents are used, the system splits into two phases. Usually the enzyme remains in the aqueous phase, so that its immediate molecular environment is not much different from that of a homogeneous aqueous reaction system. The substrate can be added to the organic phase and is transferred across the interface into the aqueous phase, where the enzymatic reaction takes place. Subsequently, the products are extracted in situ into the organic phase (Halling, 1994; Klibanov et al., 1977). Thus, the organic phase acts both as substrate reservoir and in situ extraction solvent. This has several advantages:

- Higher substrate concentrations can be supplied, which increases the volumetric productivity (Fernandes and Cabral, 2008; Gröger et al., 2004; Villela Filho et al., 2003). 
- Higher product concentrations can be obtained, which reduces the amount of solvent removal needed for product purification.

- The removal of organic solvents is usually cheaper than that of water due to lower evaporation enthalpies.

- The use of organic solvents allows an easier integration of the enzymatic process step into an chemical process, which is typically based on organic solvents.

- The enzyme can be easily retained and reused (Fernandes and Cabral, 2008; Halling, 1987; Sheldon, 2005).

- Undesired consecutive or side reactions can be avoided (Gerrits et al., 2001; Villela Filho et al., 2003).

- Inhibitions of the enzyme due to excess substrate or product can be suppressed (Diender et al., 2002; Rosche et al., 2004).

- The equilibrium conversion and product yield can be significantly higher compared to homogeneous systems since the in situ product extraction into the non-reactive phase can shift the equilibrium position (Diender et al., 2002; Eckstein et al., 2006a; Peters et al., 2007).

The interface between the aqueous and the organic phase can play an important role. Some enzymes, e.g. several lipases, are activated when adsorbed on the interface (Fernandes and Cabral, 2008; Straathof, 2003). Conversely, enzyme denaturation can often be observed at the interface (Baldascini and Janssen, 2005). Apart from this deactivation mechanism, which is known as phase toxicity, also molecular toxicity can occur in aqueous-organic biphasic systems. The latter is caused by organic molecules dissolved in the aqueous phase which interact directly with the enzymes (Butler, 1979; Halling, 1994; Villela Filho et al., 2003).

In order to avoid enzyme denaturation due to phase toxicity, the immobilization of enzymes may be beneficial. In this way, the direct contact of enzymes with the organic solvent is minimized and, therefore, their stability is increased (Cao, 2005; Fernandes and Cabral., 2008; Mateo et al., 2007; Sheldon, 2007). Since heterogeneous catalysts can easily be recovered and reused, enzymes are mostly utilized in immobilized form for industrial applications (Schmid et al., 2001, 2002; Sheldon, 2007). Furthermore, the immobilization in carriers can improve the dispersibility and mass transfer as a result of increased surface area (Halling, 1994). Thus, continuous reactors can be designed using either a fluidized bed or a packed bed (Ansorge-Schumacher et al., 2006; Sheldon, 2007). One possibility is the 
immobilization in hydrogel beads (Ansorge-Schumacher, 2007; Ansorge-Schumacher et al., 2000; Hischer et al., 2005; Metrangolo-Ruiz de Temiño et al., 2005). Due to their high water content, hydrogels possess an excellent biocompatibility (Kopecek and Yang, 2007).

\subsection{Mechanistic modeling of enzyme immobilizates}

The rational design of biphasic systems is in general more complex compared to homogeneous systems since mass transfer effects have to be considered (Bauer et al., 2002; Diender et al., 2002; Halling, 1994). If the enzymes are immobilized in hydrogel beads, diffusion has to be taken into account (Berendsen et al., 2006; van Roon et al., 2006). Therefore, in a gel-stabilized reaction system, as depicted in Figure 1-1, three superimposed phenomena take place: mass transfer of the reactants through the interface, diffusion inside the hydrogel bead, and enzyme reaction.

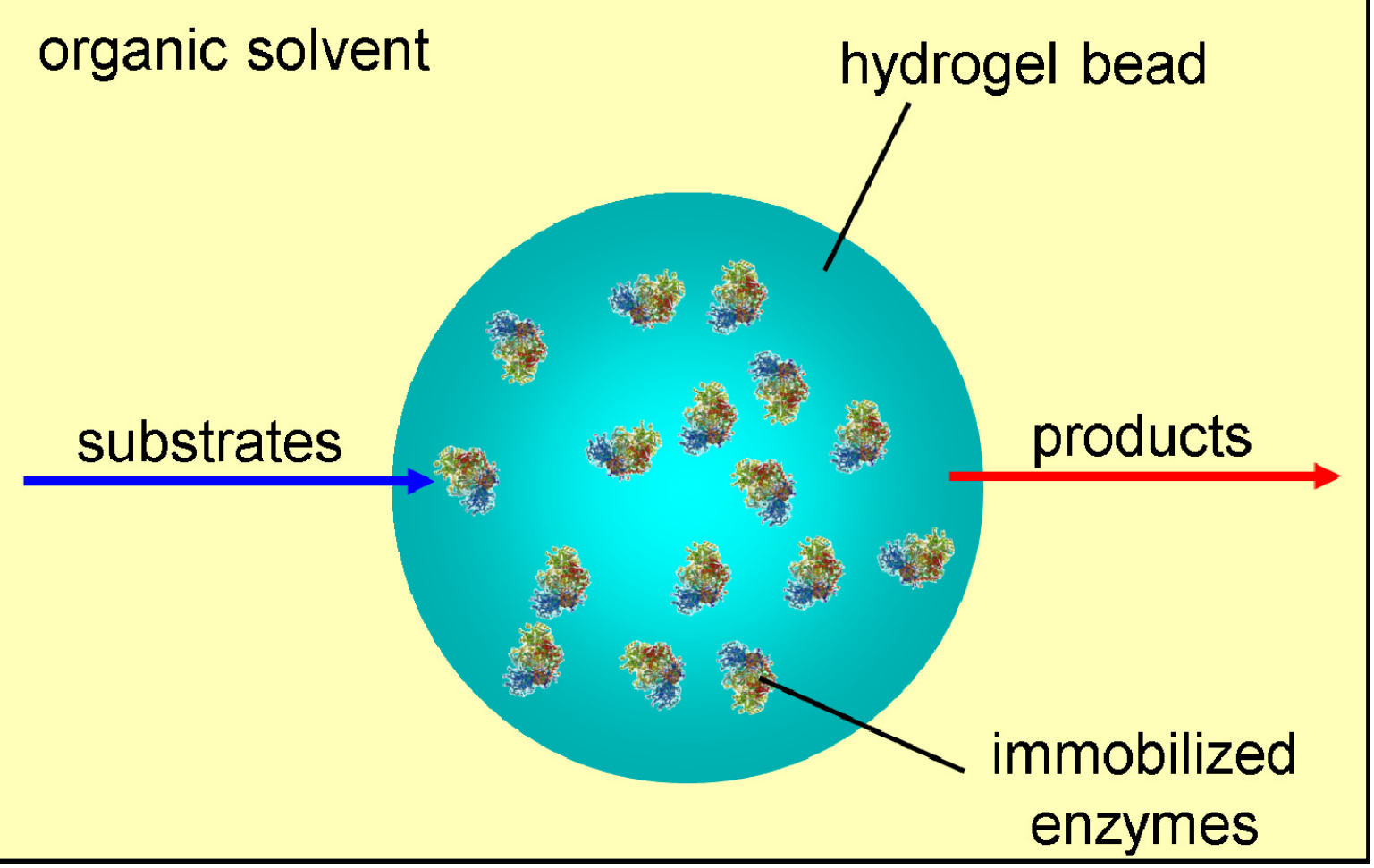

Figure 1-1: Gel-stabilized aqueous-organic biphasic reaction system.

Due to this complexity, the rational design of such systems is difficult and usually performed empirically, despite experience with immobilized enzymes for more than 40 years (Buchholz et al., 2005). However, empirical optimization requires many experiments and makes it 
difficult or even impossible to discriminate between possible reasons for limitations occurring in the system. Moreover, until today experimental data have been almost exclusively collected from the bulk phase (Berendsen et al., 2006; Goncalves et al., 2008; Polakovic et al., 2001; Schroen et al., 2002; van Roon et al., 2006). These data can provide only integral information about the reaction system and hardly allow any conclusions about the interdependent phenomena occurring inside the bead (Heinemann, 2003). To overcome this limitation, highly resolved measurement techniques should be applied, which allow to quantify concentrations inside the hydrogel bead. Moreover, for a rational design of such immobilizates models with lumped pseudo-kinetic constants (e.g. Schroen et al., 2002) are not suitable. Instead, a mechanistic kinetic model based on partial differential and algebraic equations which accounts for all occurring phenomena is required.

Dynamic mechanistic kinetic modeling has become a key activity in process engineering and is considered to be increasingly important in the chemical industry (Asprey and Macchietto, 2000; Berger et al., 2001; Franceschini and Macchietto, 2007). The main reason is that mechanistic kinetic modeling assures the correctness of the model prediction if the model is extrapolated (Franceschini and Macchietto, 2008d). Thus, it can be applied for model-based process design, optimization, control, operation, and scale-up of processes without the need to perform a very large range of experiments (Bardow and Marquardt, 2007; Berger et al., 2001; Willeman et al., 2002a, 2002b). This is especially important for the production of hydrophobic fine chemicals, where a high performance process is essential (Blaser, 2003). Besides these advantages for process engineering, mechanistic kinetic modeling of enzymatic reactions also contributes to an increased knowledge about the enzyme mechanism (VasicRacki et al., 2003; Vrsalovic Presecki et al., 2006). For example, Franco et al. (2008) have demonstrated that mechanistic kinetic modeling can explain the enhanced performance of pulsed bioreactors. However, the mechanistic modeling of (bio-)chemical reactions in homogeneous and heterogeneous systems is still one of the most relevant and yet not satisfactorily solved tasks in process engineering (Berger et al., 2001).

\subsection{Model-based experimental analysis}

A systematic work process for the identification of mechanistic models of complex kinetic phenomena in multi-phase reaction systems was published by Marquardt (2005). This concept 
is known as "model-based experimental analysis" (MEXA) and aims at systematically improving both model structure and experimental techniques within an iterative cycle. The approach is similar to the procedure proposed by Asprey and Macchietto (2000). Within the Collaborative Research Centre 540 at the RWTH Aachen University the MEXA work process is applied to investigate several challenging kinetic phenomena such as reaction kinetics in multi-phase systems, multi-component diffusion, as well as mass and heat transfer in falling films (Marquardt, 2005). The basic steps of the MEXA method are illustrated in Figure 1-2.

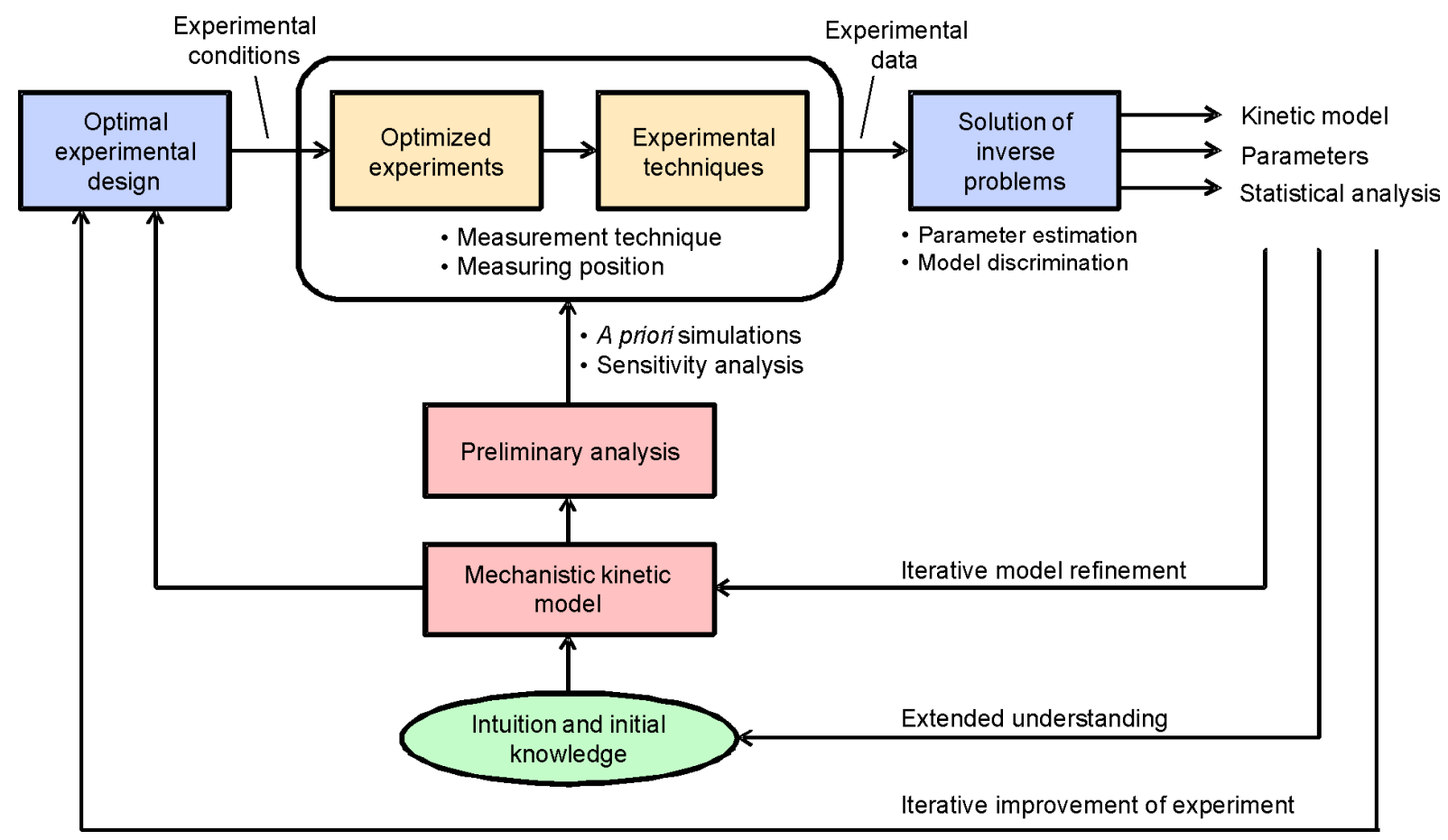

Figure 1-2: $\quad$ Work process of the model-based experimental analysis (MEXA) approach (adapted from Marquardt (2005)).

\subsubsection{Mechanistic kinetic modeling and preliminary analysis}

The MEXA approach begins with the development of a mechanistic kinetic model before any experiments are conducted. For the model development intuition and initial knowledge such as physical, chemical, or biological laws are employed. Sometimes this results in model variants (Asprey and Macchietto, 2000; Franceschini and Macchietto, 2008c; Marquardt, 2005). 
On the basis of the developed mechanistic model or model variants a priori simulations can be performed in order to decide for a suitable experimental setup and to explore the expected behavior of the experiment (Bardow and Marquardt, 2007; Marquardt, 2005). Moreover, it is important to evaluate how the model parameters influence the predicted output trajectories of the model. This can be achieved by means of a local sensitivity analysis. A sensitivity analysis is useful to identify the most suitable time intervals to collect data for the parameter estimation. Furthermore, it reveals the most sensitive model parameters as well as those which are not identifiable under the conditions applied (Beck and Arnold, 1977). The identifiability of model parameters should always be tested prior to parameter estimation (Walter and Pronzato, 1990). In multi-phase systems a sensitivity analysis can also assist in identifying the optimal measuring position. Parameter sensitivities can be obtained via finite differences, analytically (Bard, 1974; Walter and Pronzato, 1990), or via automatic differentiation (Vehreschild, 2004).

The mechanistic model can also be employed to conduct in silico experiments. For this purpose, the model is used to simulate data which are subsequently disturbed with white noise. In silico experiments are useful to check the identifiability of kinetic parameters, to compare different regularization strategies (Santos and Bassrei, 2007), to determine the discriminability between model variants (Burke et al., 1997; Kremling et al., 2004), to validate new model identification approaches (Brendel et al., 2006), or to evaluate the precision of analysis programs (Straathof, 2001).

\subsubsection{Optimal experimental design and measurement techniques}

Based on the mechanistic model optimal experiments can be designed (Bardow and Marquardt, 2007; Walter and Pronzato, 1990). Pioneering work on model-based optimal experimental design (OED) was done by Box and Lucas (1959). OED improves the precision of parameter estimates by maximizing the information content of the designed experiments (Bauer et al., 2000). The principle is to minimize the variance-covariance matrix using a design criterion such as A-, D-, or E-optimality. These criteria minimize the trace, the determinant, and the largest eigenvalue of the variance-covariance matrix, respectively. This can be geometrically interpreted as minimizing the box around a confidence ellipsoid, its volume, or the size of its major axis (Asprey and Macchietto, 2000; Walter and Pronzato, 1990). Recently, novel anticorrelation criteria which aim at minimizing correlations of 
parameters were formulated by Franceschini and Macchietto (2008c) and applied to a biodiesel production process (Franceschini and Macchietto, 2008b). OED minimizes the number of experiments, and, therefore, the costs of materials and the time required. Thus, OED is increasingly applied in industry and academia (Arellano-Garcia et al., 2007; Franceschini and Macchietto, 2007, 2008a). For non-linear models the design depends on the initial guess for the unknown parameters. The closer these initial values approach the true values, the better the obtained OED will be. OED is, therefore, an iterative procedure (Box and Lucas, 1959; Cappuyns et al., 2007; Franceschini and Macchietto, 2008d; Walter and Pronzato, 1990). OED can also be employed for designing parallel experiments (Galvanin et al., 2007). Only few applications of OED for the design of enzymatic experiments exist (Duggleby and Clarke, 1991; Lindner and Hitzmann, 2006; Murphy et al., 2003). Moreover, these studies mostly focused on simple Michaelis-Menten equations and none of them was used to design optimal experiments in biphasic systems.

OED can be used not only to improve parameter precision, but also to discriminate between model variants. This application was first investigated by Hunter and Reiner (1965). The principle is to determine experimental conditions under which the difference between the predictions of alternative model variants is maximized. Later on, this concept was further improved and investigated in detail (Asprey and Macchietto, 2000; Atkinson and Fedorov, 1975a, 1975b; Burke et al., 1997; Draper and Hunter, 1966; Hill, 1978; Kremling et al., 2004; Walter and Pronzato, 1990). In all cases OED requires interactions between modeler and experimenter since a compromise between maximum information content and practical feasibility has to be achieved (Cappuyns et al., 2007).

For the purpose of parameter estimation and model discrimination highly resolved measurement techniques are beneficial (Marquardt, 2005). Therefore, non-invasive spectroscopic techniques should be applied such as nuclear magnetic resonance (NMR), absorbance, and fluorescence. For the quantification of fluorescent molecules inside enzyme immobilizates confocal laser scanning microscopy (CLSM) enables a high spatial and temporal resolution (Spiess and Kasche, 2001). 


\subsubsection{Solution of inverse problems}

The experimental data are the outcome of unknown phenomena, which have to be quantified. Thus, this is considered as an inverse problem. Both the identification of the correct kinetic model and the estimation of the model parameters are inverse problems. One prerequisite for the solution of inverse problems is the specification of an adequate variance model for the experimental data because this affects the estimated parameter values and statistics (Franceschini and Macchietto, 2008a; Lindner and Hitzmann, 2006). Especially for dynamic parameter estimation, reasonable initial guesses and bounds should be chosen for the model parameters in order to reduce the risk to get stuck in local minima (Bard, 1974). After an estimation is performed, it is important to statistically assess the adequacy of the kinetic model and the precision of the parameter estimation. This can be done by using standard tools such as overlay and residual charts (Cornish-Bowden, 2001) and statistical tests like the Student-t-test. Especially in (bio-)chemistry, the mechanistic kinetic models are often highly non-linear and contain a large number of correlated model parameters. These correlations cause convergence problems and make it difficult to identify unique parameter values. Additionally, the obtained estimates can be inaccurate and the individual confidence intervals are not reliable. Therefore, correlations should always be taken into account in terms of e.g. the correlation matrix or confidence ellipsoids (Franceschini and Macchietto, 2007, 2008a, 2008b, 2008c). However, confidence intervals, the correlation matrix, and confidence ellipsoids are deduced from the variance-covariance matrix which is based on a linearization. Thus, the results can be unreliable for non-linear kinetic models (Kremling et al., 2004).

Often the first MEXA cycle does not quantify the occurring kinetic phenomena with sufficient detail and precision. Therefore, the extended knowledge should be used for iterative model refinement and improvement of the experimental techniques (Bardow and Marquardt, 2007; Marquardt, 2005).

\subsection{Interdisciplinary collaboration}

For the realization of the MEXA work process specialists of different research disciplines are required. Four specialists - a biotechnologist, a physicist, a process systems engineer and a biochemical engineer - collaborated for the analysis of the gel-stabilized aqueous-organic 
biphasic reaction system. Enzymatic experiments in homogeneous and biphasic systems were conducted by Thomas Schmidt (Chair of Biotechnology) and the measurements with the confocal laser scanning microscope by Tilman Schwendt (Fraunhofer Institute for Laser Technology). The OED was performed in close cooperation with Claas Michalik (Aachener Verfahrenstechnik - Process Systems Engineering), who developed methodic extensions for model identification. The development of mechanistic kinetic models, a priori simulations, sensitivity analysis, and parameter estimation were the main tasks of the author of this thesis.

\subsection{Objectives and overview}

The aim of this thesis is the analysis of the gel-stabilized aqueous-organic biphasic system described above. For this purpose, the MEXA method is applied in order to derive a mechanistic kinetic model which takes enzyme reaction, mass transfer, and diffusion into account. This increases knowledge about the occurring phenomena and their interactions and will allow the rational optimization of enzyme immobilizates. The approach within this thesis is to separate the occurring phenomena from each other in a first step, which allows to study them individually. Afterwards, these phenomena are combined successively to yield to the gel-stabilized aqueous-organic biphasic system in the end (Figure 1-3).

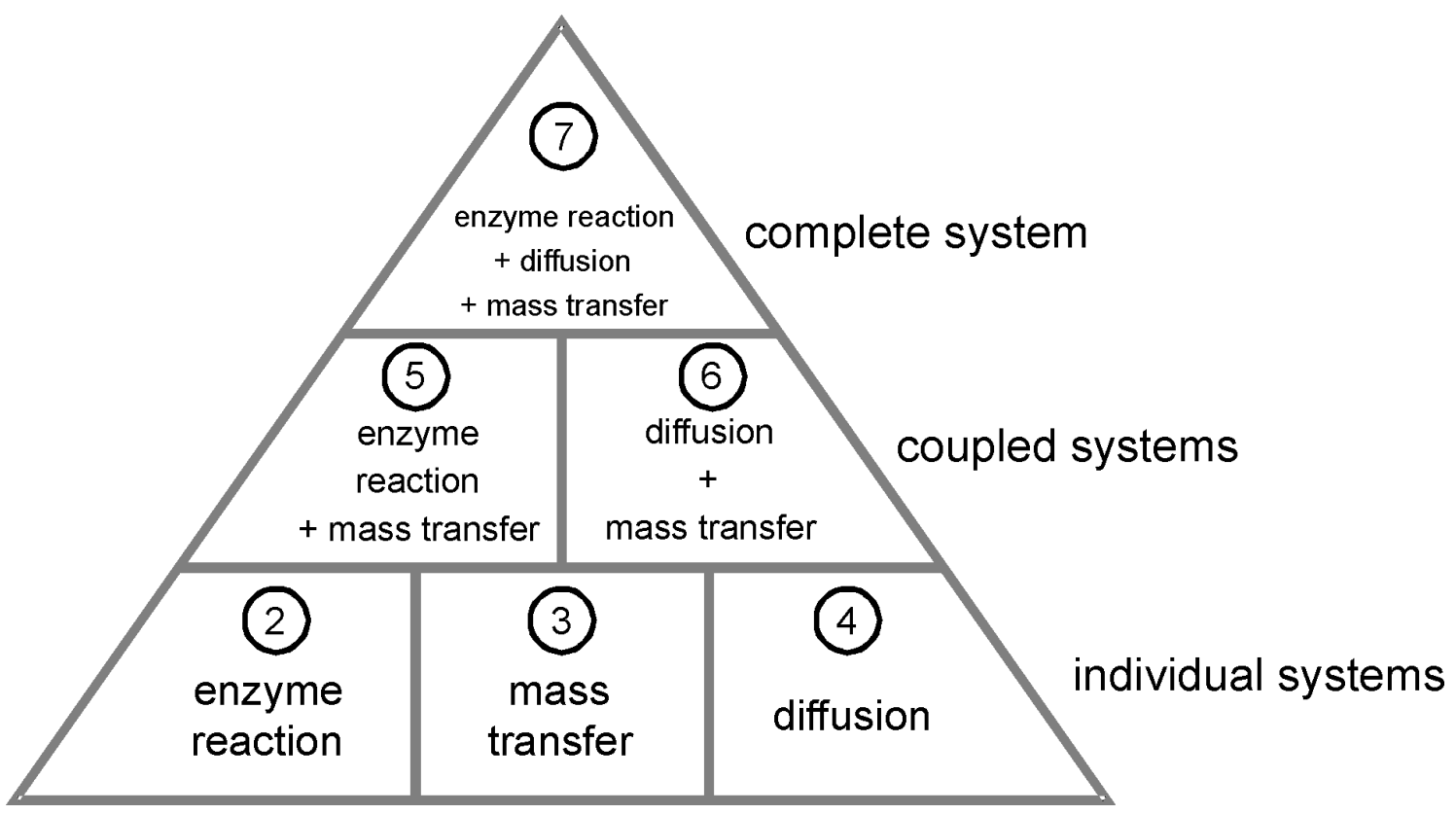

Figure 1-3: $\quad$ Separation and coupling of occurring phenomena. Each number indicates the chapter of this thesis, in which the respective system is analyzed. 
In Chapter 2 the mechanistic modeling of enzyme kinetics is addressed. At first, different approaches for progress curve analysis are presented and compared. Subsequently, a mechanistic kinetic model for benzaldehyde lyase (BAL) is derived for the first time. Additionally, the use of micro-reaction rate constants is compared to the use of macroscopic parameters for modeling enzyme kinetics. Moreover, some basic remarks are given for the OED for symmetric carboligation reactions. The obtained results are interpreted on the molecular level and compared to the homologous enzyme benzoylformate decarboxylase (BFD).

Chapter 3 deals with the mass transfer between an ideally mixed aqueous and organic phase. A new approach for solvent selection based on ab initio calculations is presented. The diffusion in hydrogel beads is investigated in Chapter 4. There, the diffusion models of Fick and Nernst-Planck are discriminated based on the example of propionic acid diffusion into alginate beads. Moreover, the diffusion of the reactants of the BAL reaction in $\kappa$-carrageenan hydrogel is studied.

The coupling of enzyme reaction and mass transfer is the topic of Chapter 5. There, not only separated phases are examined, but also technically more relevant emulsion systems. In Chapter 6 the coupling of diffusion and mass transfer is addressed. Finally, Chapter 7 focuses on the gel-stabilized aqueous-organic biphasic system. Limitations due to diffusion or reaction are discussed. Using sensitivity analysis optimal measurement strategies are derived and the obtained parameter estimates are compared to those obtained in the individual and coupled systems. A summary and outlook in Chapter 8 concludes the work. 


\section{Enzyme Kinetics in Homogeneous Aqueous Phase}

This chapter focuses on the mechanistic modeling of enzyme kinetics in homogeneous aqueous systems. First different approaches and programs for analyzing enzyme kinetics are compared based on the example of peptide bond hydrolysis using the enzyme penicillin amidase (PA) from Escherichia coli. This enzyme is well studied and thus serves as reference. On this basis, the most suitable method is determined and chosen for the subsequent modelbased experimental analysis (MEXA) of the enzyme benzaldehyde lyase (BAL) from Pseudomonas fluorescens. Since for BAL no mechanistic kinetic model is available so far, a new one is derived using the MEXA-method. This mechanistic kinetic model allows the calculation of micro-reaction rate constants, which provide valuable information about the enzyme mechanism and which in turn can be used to identify catalytic bottlenecks. Moreover, general guidelines for optimal experimental design (OED) of symmetric carboligations are given and then used for the estimation of kinetics in different cosolvents. In the end of this chapter, these results are compared to benzoylformate decarboxylase (BFD), a homologous enzyme to BAL.

\subsection{Comparison of different approaches and computer programs for progress curve analysis of enzyme kinetics}

\subsubsection{Introduction}

Modeling of enzyme kinetics does not only contribute to an increased knowledge about the enzyme mechanism, but is also essential for the design of new processes, for scale-up, and for optimization of existing processes (Vasic-Racki et al., 2003). For this purpose, accurate values of the kinetic parameters are required. Since the derivation of the Michaelis-Menten equation in 1913 several approaches for the determination of these kinetic parameters were developed. The classical approach uses initial rate measurements and graphical estimation of the kinetic parameters in terms of e.g. Lineweaver-Burk plots (Lineweaver, 1934). A serious 
drawback of these plots is the distortion of the error distribution resulting from the rearrangement of the data, which generally results in biased parameter estimates. This can be circumvented by using algebraic parameter estimation for fitting the kinetic model to initial rates. The advantage of initial rate measurements is that the analysis procedure is easy to perform, computationally cheap, and well-established. Moreover, the substrate conversion is very low, which minimizes complicating phenomena such as reverse reaction, product inhibition, or enzyme deactivation. On the other hand, initial rate measurements require a high number of individual experiments, so that large amounts of substrate and enzyme are necessary, which can be costly (Liao et al., 2005). Additionally, the calculation of reaction rates from concentration data is an inverse problem. Furthermore, initial rate measurements are very sensitive concerning noise, and phenomena occurring after longer time intervals are overlooked.

A more sophisticated approach for studying enzyme kinetics is progress curve analysis. Here, the enzyme reaction is followed by measuring the substrate or product concentration for longer times. This reduces the number of experiments since multiple data points are collected from one single experiment. In case of enantioselective conversions no experiments with initial enantiomerically pure product are required since it is formed by the enzyme (Duggleby, 2001). Progress curve analysis can be used for model discrimination (Bates and Frieden, 1973; Michalik et al., 2007) and for detection of competitive inhibition (Gutierrez and Danielson, 2006a). Furthermore, it reveals long-time phenomena like enzyme deactivation which are of special interest for the design of industrial processes (Straathof, 2001). Nevertheless, progress curve analysis also exhibits several disadvantages. Besides unknown chemical side reactions the error propagation due to inaccurate initial concentrations and displacements in starting time assignment is one inherent drawback (Duggleby, 2001, Franco et al., 1991). However, solutions to this problem have been developed, such as the use of sophisticated variance models (Gutierrez and Danielson, 2006b), the use of a weighting matrix (Franco et al., 1991; Markus et al., 1981), or the estimation of the initial concentrations (Newman et al., 1974, Rakels et al., 1994).

The most important problem in progress curve analysis is the numerical complexity of the fitting procedure (Duggleby and Daniel, 1995). While the kinetic laws are formulated as rates the experimental data are collected in terms of concentrations. For batch processes in homogeneous aqueous systems with constant volumes and one single reaction, the rate of a 
reactant equals the temporal derivative of the respective concentration. Thus, either the measured concentrations have to be differentiated (Yeow et al., 2003, 2004) or the kinetic law has to be integrated (Liao et al., 2005) before algebraic parameter estimation can be performed. The calculation of reaction rates from noisy concentration versus time data constitutes an ill-posed problem in the sense of Hadamard (1923) since not one unique solution exists which depends continuously on the data in some reasonable topology. Thus, small errors are amplified through differentiation. In other words, the noise-level is significantly increased if e.g. finite differences are applied for differentiation. Thus, small concentration errors may cause large rate errors. To avoid this, adequate regularization techniques are necessary (Brendel et al., 2006; Marquardt, 2005). One regularization option is to find a smooth function which fits the concentration data and which subsequently can be differentiated to obtain a rate with a low noise level (Bardow and Marquardt, 2004b). Possible regularization techniques are e.g. filter-based approaches (Kalman, 1960), Fourier transformation-based approaches (Yablonsky et al., 2007), or Tikhonov regularization (Yeow et al., 2003, 2004). A special case of the general Tikhonov formulation is the use of smoothing splines (de Boor, 1978; Reinsch, 1967). All regularization techniques have in common that a compromise has to be found between staying close to the original data and minimization of noise (Hansen, 1998). The alternative to the differentiation of the concentrations is the integration of rate equations. This can be achieved by integrating the rate equations analytically (Liao et al., 2005). However, this results even for very simple rate equations in very complex expressions involving a number of simplifying assumptions. For more complex reactions this analytical integration is not applicable any more. Therefore, this procedure is not generally applicable (Rakels et al., 1994).

For both approaches described above, differentiation of the concentration data or analytical integration of the rate equation, subsequent algebraic parameter estimation can be applied. An alternative to both approaches for studying enzyme kinetics is the use of dynamic parameter estimation, where the kinetic law is repeatedly integrated numerically (Bates and Frieden, 1973; Marokhazi et al., 2004). The advantage is that neither the rate equation nor the concentration data have to be modified by the user and statistically sound results can be obtained. Therefore, it is a generally applicable and flexible method for progress curve analysis (Rakels et al., 1994). However, dynamic parameter estimation is computationally expensive, and often only local instead of global minima of the objective function are found, if the initial guesses for the kinetic parameters are poor and the kinetic model is complex 
(Marquardt, 2005). An alternative is the incremental approach (Bardow and Marquardt, 2004b; Brendel et al., 2006). This approach starts with algebraic parameter estimation for model discrimination and for parameter initialization. The obtained initial parameter estimates are used for subsequent dynamic parameter estimation. Michalik et al. (2007) applied this incremental method to progress curve analysis of enzymatic reactions for the first time.

Today numerous programs exist which can be used for progress curve analysis (Copeland, 2000). One group of programs makes use of algebraic parameter estimation, while others are able to perform dynamic parameter estimation. In the last 30 years a large amount of surveys were published demonstrating that different computer programs can produce different results (e.g. Altman et al., 2004; McCullough, 1998, 1999; McCullough and Wilson, 1999). Although this drawback is well known by professional data analysts and statisticians, it is widely ignored in the field of enzymology. The program choice for analyzing enzyme kinetics is usually rather subjective and often depends on the availability of the software. To date it is often assumed that the program choice does not affect the obtained values for enzyme kinetic parameters. In this Section the importance of the program choice for the analysis of enzyme kinetics is highlighted by fitting experimental and in silico data using five different computer programs, which are based on either algebraic or dynamic parameter estimation.

\subsubsection{Materials and methods}

\subsubsection{Experimental}

Progress curve analysis was performed on the example of peptide bond hydrolysis using penicillin amidase (PA, EC 3.5.1.11) from Escherichia coli (Sigma-Aldrich, Deisenhofen, Germany). The studied reaction was the hydrolysis of the chromogenic substrate 6-nitro-3phenylacetamido benzoic acid (NIPAB) to 5-amino-2-nitrobenzoic acid (ANB) and phenylacetic acid (PAA). The applied assay contained $50 \mathrm{mM} \mathrm{K \textrm {H } _ { 2 }} \mathrm{PO}_{4} / \mathrm{K}_{2} \mathrm{HPO}_{4}$ buffer of $\mathrm{pH}$ 7.5 with an ionic strength of $200 \mathrm{mM}$. The molar concentration of active enzyme was determined using an active site titration using phenylmethylsulfonyl fluoride according to Svedas et al. (1977). All chemical substances were of analytical grade and purchased from Sigma-Aldrich (Deisenhofen, Germany). The reaction was conducted in standard photometric cuvettes with a filling volume of $1 \mathrm{~mL}$ and started by addition of NIPAB. The temperature was kept constant at $25^{\circ} \mathrm{C}$ using a thermostated cuvette holder. Mixing was achieved by using 
a magnetic stir bar. The increase in absorbance at $405 \mathrm{~nm}$ due to the formed ANB (Alkema et al., 1999) was measured using an Uvikon 922 spectrophotometer (Kontron Instruments, GroßZimmern, Germany). In total nine experimental progress curves were measured (Table 2-1).

Table 2-1: Initial conditions for each experiment. The concentrations of ANB and active enzyme were always $0 \mathrm{mM}$ and $2.86 \cdot 10^{-4} \mathrm{mM}$, respectively.

\begin{tabular}{lll}
\hline Experiment & NIPAB [mM] & PAA [mM] \\
\hline A & 0.075 & 0 \\
B & 0.1 & 0 \\
C & 0.15 & 0 \\
D & 0.1 & 0.05 \\
E & 0.1 & 0.075 \\
F & 0.1 & 0.1 \\
G & 0.1 & 0.2 \\
H & 0.1 & 0.3 \\
I & 0.1 & 0.5 \\
\hline
\end{tabular}

\subsubsection{Kinetic model}

The enzymatic reaction follows a ping-pong bi-bi mechanism. In aqueous solution water can be considered to be in excess, so that it cannot be distinguished from an ordered uni-bi mechanism. PAA acts as a competitive inhibitor. Under the applied conditions the reaction is considered to be irreversible (Kheirolomoom et al., 2001, Warburton et al., 1973). The reaction can be modelled with Eq. 2-1 (Alkema et al., 1999; Cleland, 1963):

$$
v=-\frac{d c_{A}}{d t}=\frac{d c_{P}}{d t}=\frac{d c_{Q}}{d t}=\frac{k_{c a t} \cdot c_{A}}{K_{m A} \cdot\left(1+\frac{c_{Q}}{K_{i Q}}\right)+c_{A}} \cdot c_{E}
$$

In Eq. 2-1 $c_{A}, c_{P}$, and $c_{Q}$ denote the concentrations NIPAB, ANB, and PAA, respectively. $c_{E}$ stands for the enzyme concentration, $k_{c a t}$ for the maximum turnover number, $K_{m A}$ for the Michaelis constant of the substrate, and $K_{i Q}$ for the inhibition constant of PAA. 


\subsubsection{In silico experiments}

Based on the kinetic model simulations were conducted with the same initial conditions as for the photometric experiments (Table 2-1). For the simulation realistic values stemming from preliminary investigations were chosen for the kinetic parameters (Table 2-2). Subsequently, the nine simulated progress curves were disturbed with Gaussian noise with a constant standard deviation of $0.002 \mathrm{mM}$. The obtained progress curves were then treated as "real" data for parameter estimation.

Table 2-2: $\quad$ Preset values of the kinetic parameters for the in silico experiments.

\begin{tabular}{lcl}
\hline Parameter & Value & Unit \\
\hline$k_{\text {cat }}$ & 10.260 & $\mathrm{~s}^{-1}$ \\
$K_{i Q}$ & 0.248 & $\mathrm{mM}$ \\
$K_{m A}$ & 0.033 & $\mathrm{mM}$ \\
\hline
\end{tabular}

\subsubsection{Influence of the amount of data}

The influence of the amount of data on the parameter estimation accuracy was investigated by reducing the number of measurement points of both experimental and in silico progress curves. Experimental progress curves with 6, 12, 25, and 48 data points, and in silico progress curves with $5,10,20$, and 40 data points were obtained and used for parameter estimation afterwards.

\subsubsection{Differentiation of concentration data}

If dynamic parameter estimation is applied, the raw concentration data can be used without modifications. In contrast to this, algebraic parameter estimation requires the differentiation of concentrations to rates. Two methods were used for this differentiation. The first one was finite differences

$$
\frac{d c}{d t} \approx \frac{c\left(t_{i+1}\right)-c\left(t_{i}\right)}{t_{i+1}-t_{i}}
$$

where $c\left(t_{i}\right)$ denotes the measured concentration at time point $t_{i}$. An alternative to finite differences is the analytical differentiation of smoothing splines. Therefore, cubic smoothing 
splines $\left(c_{\text {smooth }}\right)$ were fitted to the concentration data $\left(c_{\text {exp }}\right)$ according to Eq. 2-3 (de Boor, 1978, Reinsch, 1967):

$$
c_{\text {smooth }}(t)=\operatorname{argmin}\left(\lambda \cdot \sum_{i=1}^{n}\left(c_{\exp }\left(t_{i}\right)-c_{\text {smooth }}\left(t_{i}\right)\right)^{2}+(1-\lambda) \cdot \int\left|\frac{d^{2} c_{\text {smooth }}}{d t^{2}}\right| d t\right)
$$

The first term in Eq. 2-3 quantifies the difference between the smoothing spline and the data points, the second the roughness of the obtained curve. A value for the regularization parameter $\lambda$ close to 1 results in a cubic spline which fits each data point, but does not reduce noise. If $\lambda$ approaches 0 , a straight line is obtained. Therefore, the minimization of Eq. 2-3 establishes a compromise between proximity to the data points and smoothness of the fitted curve (de Boor, 1978). The smoothing splines were obtained using the curve fitting toolbox of Matlab (version 7.5, The MathWorks Inc., Natick, MA, USA). Matlab calculates the regularization parameter $\lambda$ automatically by equalling the traces of the first and the second term of Eq. 2-3. The fits were plotted to check if the deviation between the smoothing spline and the data points are physically realistic, which means that it must be in the range of the standard deviation (Yeow et al., 2004). Subsequently, the smoothing spline was differentiated analytically. This way, rates were obtained. Moreover, the smoothing spline and the reaction stoichiometry (Eq. 2-1) were used to get the respective concentrations of NIPAB, ANB, and PAA. Thus, a matrix consisting of the rate and all concentrations was built up for each progress curve, which was then utilized for the algebraic parameter estimation.

\subsubsection{Computer programs}

From the large amount of possible analysis software, five popular computer programs were selected for the comparison. These computer programs range from simple and broadly available programs to very sophisticated programs. They were used to fit the kinetic model (Eq. 2-1) to either the nine experimental or the nine in silico progress curves. For the comparison the same data, the same initial conditions (Table 2-1), the same initial guesses for the parameter estimates $\left(k_{\text {catf }}: 10 \mathrm{~s}^{-1} ; K_{m A}: 0.01 \mathrm{mM} ; K_{i Q}: 0.05 \mathrm{mM}\right)$, and the same standard deviation of measurement points $(0.002 \mathrm{mM})$ were used. For each program the respective default settings for integration and parameter estimation were applied. In the following the five programs are introduced: 
1) MS-Excel: MS-Excel (version 2003, Microsoft Corporation, Redmond, WA, USA) belongs to the most common computer programs worldwide. This general purpose calculation tool can be employed also for progress curve analysis of enzyme reactions. Using the solver add-in, algebraic parameter estimation was performed. The weighted sum of squares was calculated and minimized using the Newton method. Since the values of the kinetic parameters are of different magnitudes, the parameters were iteratively scaled to 1.0. The solver add-in does not provide any statistical results such as standard deviations.

2) Origin: Origin (version 7.5, OriginLab Corporation, Northhampton, MA, USA) is a scientific graphing and analysis software. Like MS-Excel, Origin allows algebraic parameter estimation. Minimization of the weighted sum of squares is achieved using the Simplexmethod. In contrast to MS-Excel standard deviations can be obtained.

3) Encora: Encora (version 1.2, Delft University of Technology, The Netherlands) is a specific program for progress curve analysis of enzyme kinetics (Straathof, 2001). The user can select enzymatic mechanisms from reversible and irreversible reactions with up to two substrates and products, with optional degradation of reactants and enzyme deactivation. Subsequently, the program fits the corresponding kinetic model to the experimental progress curves in terms of dynamic parameter estimation. The differential equations are integrated using a fourth-order Runge-Kutta routine. Encora applies the Simplex-like algorithm of Nelder and Mead to minimize the sum of squared residuals. The parameter estimates are provided without standard deviations.

4) ModelMaker: ModelMaker (version 3.0.4, Cherwell Scientific, Oxford, UK) exhibits a graphical user interface on which systems of ordinary differential equations can be implemented (Citra, 1997). The kinetic model is fitted in terms of dynamic parameter estimation. Integration is achieved using the fourth-order Runge-Kutta routine. The weighted sum of squared residuals is minimized via the Levenberg-Marquardt algorithm. In order to fit several progress curves with different initial conditions simultaneously, for each progress curve one identical model was implemented and the kinetic parameters were defined globally. The obtained results include standard deviations.

5) gPROMS: The software package gPROMS (version 3.0.2, Process System Enterprise Ltd., London, UK) allows dynamic parameter estimation for systems with embedded ordinary or 
partial differential equations. Due to its versatility it can be applied for complex scientific or technical applications. Integration of differential-algebraic equations is performed using the "DASOLV" solver, which is based on variable time-step/variable-order backward differentiation. Optimal parameter estimates in a maximum likelihood framework are obtained using the parameter estimation facilities of gPROMS. The parameter estimates are displayed together with a detailed statistical analysis including standard deviations, confidence ellipsoids, and residual plots.

\subsubsection{Results and discussion}

Penicillin amidase (PA) was discovered by Sakaguchi and Murao (1950). It consists of two subunits and has one active site (Duggleby et al., 1995). PA hydrolyzes penicillin G to phenylacetic acid and 6-aminopenicillanic acid, which enables the production of semisynthetic $\beta$-lactam antibiotics (Bruggink et al., 1998, Cole, 1969). Although PA has intensively been studied due to this industrial importance (e.g. Alkema et al., 2000, Arroyo et al., 2003, Giordano et al., 2006, Kheirolomoom et al., 2001), published parameter estimates vary drastically (Alkema et al., 1999). To investigate if such deviations may be caused by using different analysis approaches, PA was chosen for the comparison of the computer programs.

\subsubsection{Progress curves}

For comparison purposes, both experimental and in silico progress curves are analyzed. In silico experiments, which are simulations with subsequent noising of the data, are useful to check the identifiability of kinetic parameters, to determine the discriminability between model variants (Burke et al., 1997; Kremling et al., 2004), to validate new model identification approaches (Brendel et al., 2006), or to evaluate the precision of computer programs (Straathof, 2001). The advantage of in silico experiments is that in contrast to experimental data it can easily be checked if the parameter estimation yields the correct values.

The obtained experimental and in silico progress curves are presented in Figure 2-1. It can be seen that full conversion is reached and that higher initial NIPAB concentrations extend the time span of linear ANB formation (Figure 2-1a), while initial PAA concentrations inhibit the enzyme (Figure 2-1c). The noise level of the in silico experiments (Figure 2-1b,d) is much 
higher compared to the experimental progress curves due to the chosen standard deviation of $0.002 \mathrm{mM}$ (Section 2.1.2.3).

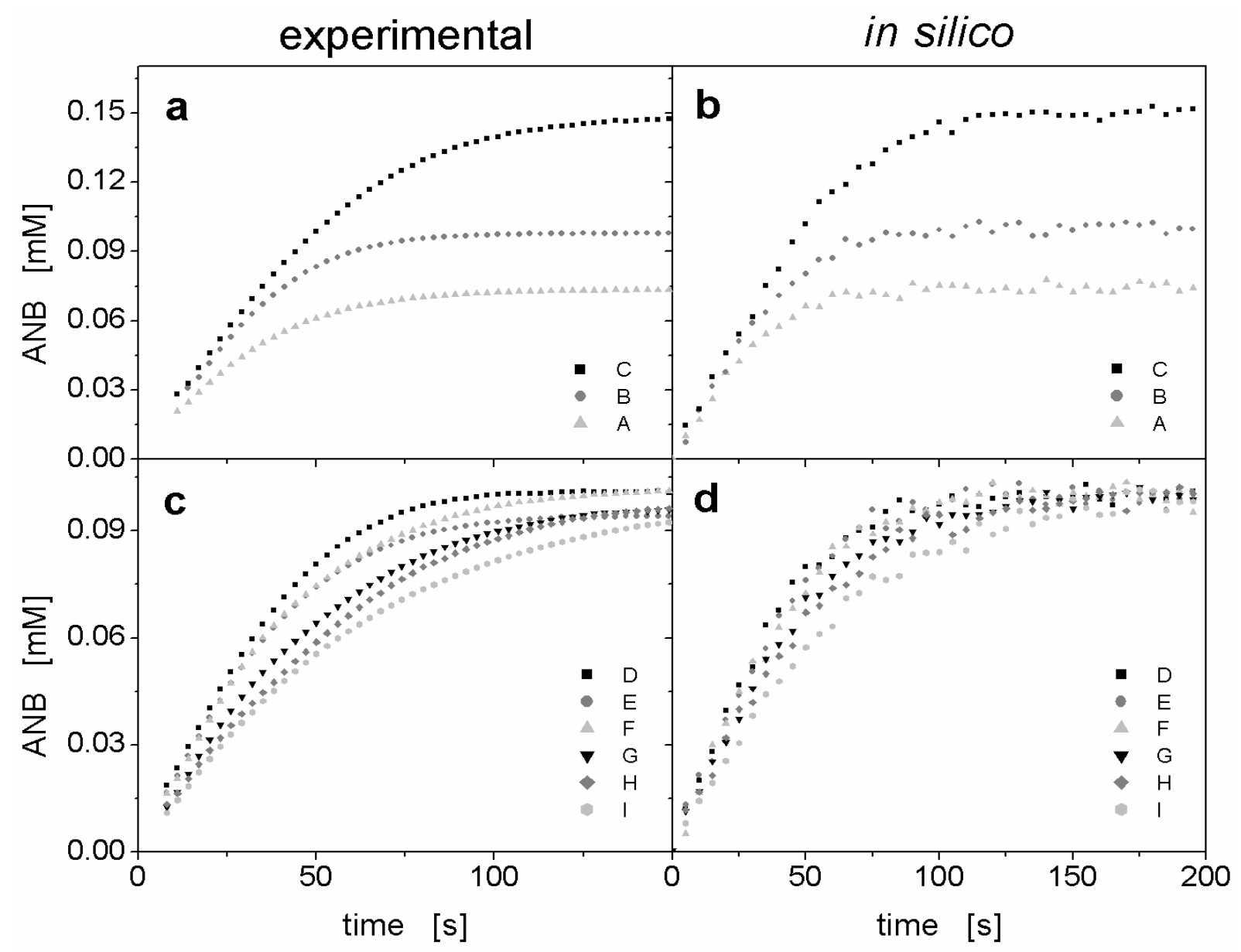

Figure 2-1: $\quad$ Progress curves with different initial concentrations of NIPAB (experimental: a; in silico: b) and PAA (experimental: c; in silico: $\mathrm{d}$ ). The letters in the legend refer to the experiments listed in Table 2-1.

\subsubsection{Differentiation of concentration data}

The calculation of reaction rates from concentration data requires adequate regularization. This is demonstrated in Figure 2-2. As it can be seen the concentration data in Figure 2-2a exhibit minor noise. However, if no regularization is applied and instead finite differences are used, the resulting curve for the reaction rate shows a high noise level (Figure 2-2b). 


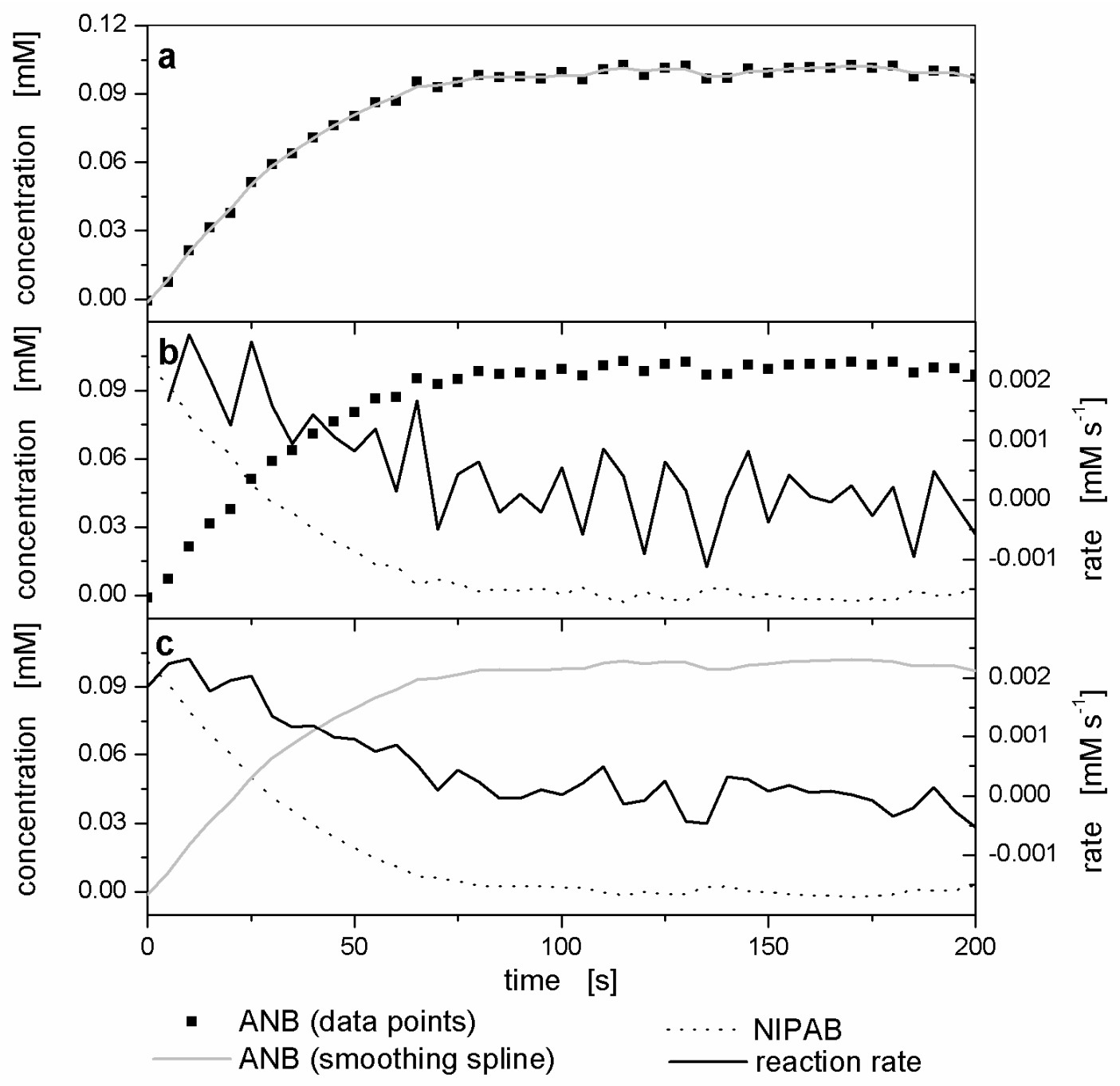

Figure 2-2: $\quad$ Progress curve of in silico experiment $B$ with the fitted smoothing spline (a); calculated reaction rate using finite differences (b) or differentiated smoothing splines (c). The NIPAB concentration was calculated using the raw data (b), or the smoothed data (c).

The better alternative is the use of smoothing splines. The smoothing spline, shown in Figure 2-2a, demonstrates the trade-off between accuracy and smoothness. Although the spline does not fit all data points exactly, no systematic deviation can be seen. Compared to the reaction rate obtained using finite differences, the analytically derived reaction rate in Figure $2-2 \mathrm{c}$ has significantly higher signal to noise ratios. Thus, the regularization parameter $\lambda$ obtained by Matlab can be considered to be satisfactory. However, there are more sophisticated methods to determine $\lambda$ such as generalized cross validation (Craven and Wahba, 1979) or the L-curve criterion (Hansen, 1992, 1994, 1999). 
To investigate if smoothing improves the subsequent algebraic parameter estimation, both the finite differences and the smoothing spline approach were compared using the nine in silico progress curves as data source and MS-Excel and Origin as computer program. Moreover, the influence of the data amount on the parameter estimation results is studied. Non-invasive, spectroscopic measurement techniques such as absorbance, fluorescence, IR, and Raman spectroscopy produce large amounts of data. However, in many cases the enzymologist is restricted to invasive measurement techniques like high performance liquid chromatography (HPLC) or gas chromatography (GC). In these cases only few data points per experiment are collected. Thus, different amounts of data points were used for parameter estimation.

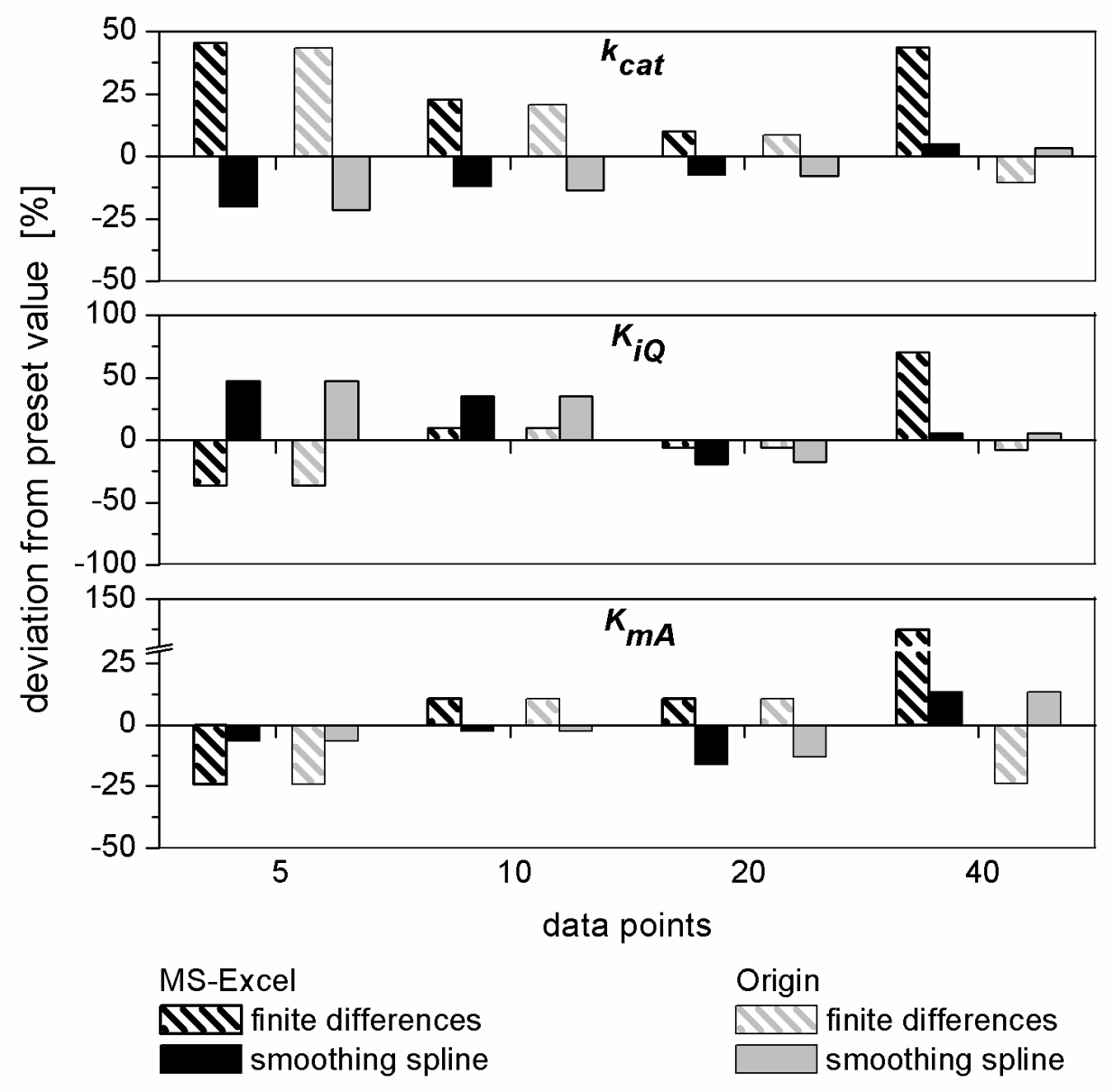

Figure 2-3: Deviations of the algebraic parameter estimates for $k_{c a t}, K_{i Q}$, and $K_{m A}$ from the preset values for the in silico progress curves. Finite differences are compared with differentiated smoothing splines using MS-Excel or Origin as computer program.

In Figure 2-3 the deviations of the obtained parameter estimates from the preset values are depicted. In 16 of 24 cases the parameter estimates, obtained using smoothing splines, were closer to the preset values than those without any regularization. In average the deviation for 
the smoothing spline approach was $16 \%$ in comparison to $26 \%$ using no regularization except for the inevitable regularization due to discretization. If 40 data points per progress curve were used, the smoothing spline method was superior in all cases. This is in agreement with Wahba (1990), who stated that smoothing splines become reliable above 25 data points, because then the inherent bias at the interval boundaries is less relevant.

Dynamic parameter estimation requires only concentrations and no smoothing of data. This was verified by comparing the results of the dynamic parameter estimation using original data with the results using smoothed data. Here, no significant effect of smoothing could be observed for 40 data points per progress curve. Compared to the use of the original data even a slight deterioration due to the regularization error was observed for lower numbers of data points (results not shown).

Based on these results it can be concluded that for the calculation of reaction rates the differentiation of smoothing splines should be preferred over finite differences, especially if a large data amount per experiment is available. However, if dynamic parameter estimation is applied, smoothing of data has no benefit.

\subsubsection{Comparison of computer programs}

As shown in Figure 2-3, the obtained parameter estimates can differ considerably from the preset values. This becomes more evident in Figure 2-4, which presents the parameter estimates obtained by the five computer programs tested. Based on the results described above, smoothing splines were used for MS-Excel and Origin, which apply algebraic parameter estimation, while for the other programs the original concentration data were utilized.

For both the experimental and the in silico progress curves large deviations among the parameter estimates exist. In general, these deviations are caused by rounding and truncation errors committed by the computer program (McCullough, 1998). The maximum difference between the lowest and the highest parameter estimates reaches $80 \%$. In general, this difference becomes smaller if more data points are used. But even for 40 data points per progress curve, differences of up to $38 \%$ are observed. This result should be kept in mind when kinetic parameter values from different publications are compared. 


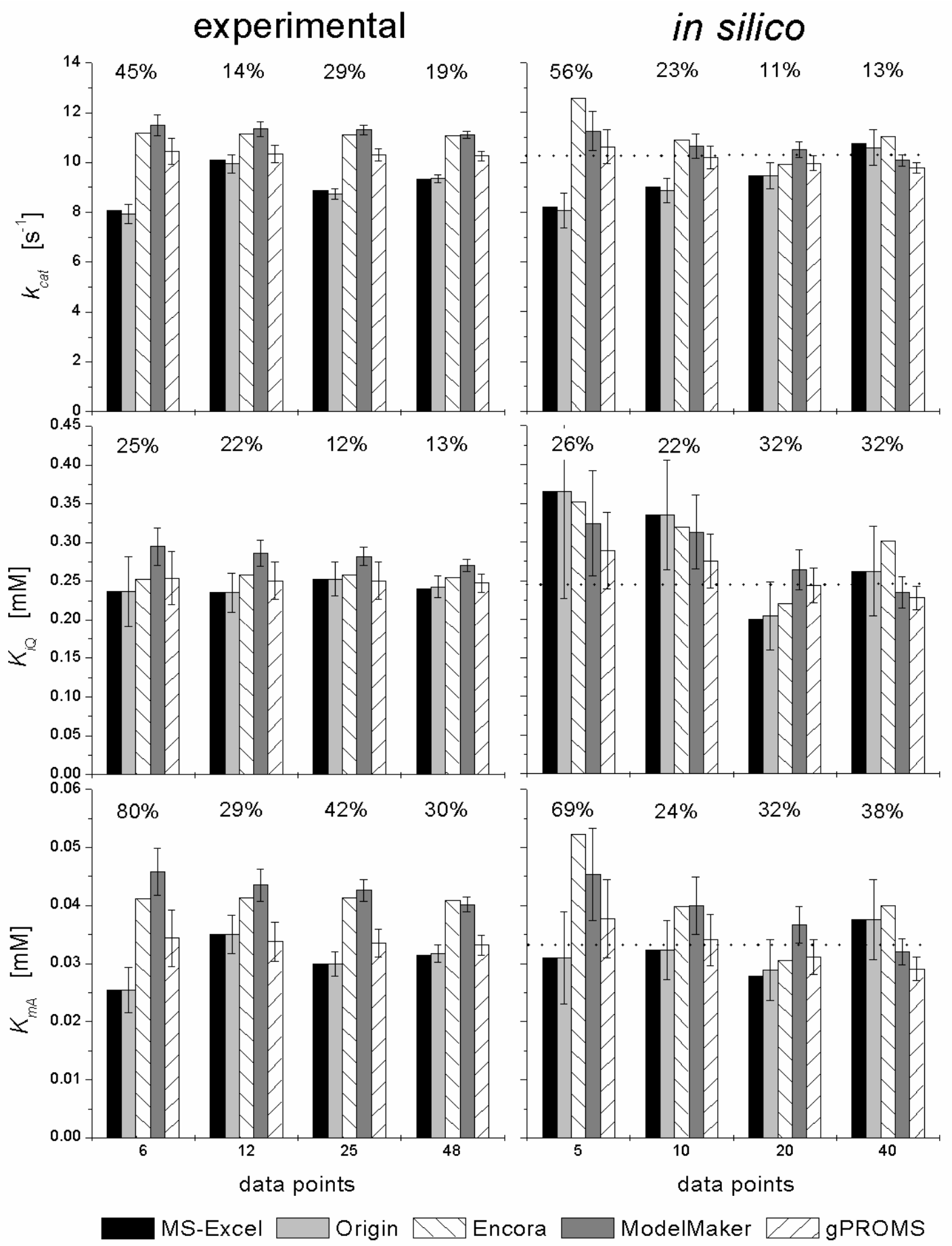

Figure 2-4: $\quad$ Parameter estimates for $k_{c a t}, K_{i Q}$, and $K_{m A}$ using experimental or in silico progress curves. The dotted lines indicate the preset values for the kinetic parameters. For each parameter and amount of data points the maximum difference among the computer programs is specified. Standard deviations are provided where applicable.

For the in silico progress curves it can be seen that the given standard deviations do not always include the preset value. However, this is typical since the probability that the true value lies within the standard deviation is only $68.3 \%$ per definition. MS-Excel and Origin, 
which both use algebraic parameter estimation, provide always very similar results. In contrast to this, larger discrepancies are found among the computer programs which apply dynamic parameter estimation. The reason for this is that these computer programs differ not only in the parameter estimation solvers, but also in their integrators. Moreover, more local minima may exist for a dynamic parameter estimation problem.

Table 2-3 compares the computer programs in terms of the averaged deviations from the preset values for the in silico progress curves. The results show that algebraic parameter estimation can compete with dynamic parameter estimation. The average deviation using MSExcel and Origin is even lower than the one of Encora. Deviations of parameter estimates from preset values for in silico experiments were already observed for Encora by Straathof (2001). ModelMaker is slightly more accurate than MS-Excel and Origin. The highest accuracy is achieved with gPROMS.

Table 2-3: $\quad$ Deviations from preset values using the computer programs (averaged over $k_{\text {cat }}, K_{i Q}$ and $K_{m A}$ ).

\begin{tabular}{lccccc}
\hline Data points & MS-Excel & Origin & Encora & ModelMaker & gPROMS \\
\hline 5 & $25 \%$ & $25 \%$ & $41 \%$ & $26 \%$ & $11 \%$ \\
10 & $16 \%$ & $17 \%$ & $19 \%$ & $17 \%$ & $5 \%$ \\
20 & $14 \%$ & $13 \%$ & $7 \%$ & $7 \%$ & $3 \%$ \\
40 & $8 \%$ & $8 \%$ & $17 \%$ & $3 \%$ & $8 \%$ \\
\hline Average & $16 \%$ & $16 \%$ & $21 \%$ & $13 \%$ & $7 \%$ \\
\hline
\end{tabular}

Certainly, the precision of the parameter estimates is the most important factor that should be considered when choosing a computer program. Nevertheless, some additional aspects are relevant. In general, the calculation time for algebraic parameter estimation is lower than for dynamic parameter estimation. However, for small models like the one used here, calculation time does not play an important role. If algebraic parameter estimation is applied, Origin should be preferred since MS-Excel does not provide standard deviations. This recommendation is in line with McCullough and Wilson (1999) who advise not to use MSExcel for statistical data analysis. Encora has not only a low precision of the parameter estimates, but requires also a special data format. Moreover, Encora supports neither the export of fitting curves nor the calculation of standard deviations. However, the advantage of Encora is that it contains a large pool of possible enzyme mechanisms the user can choose. 
Therefore, Encora could be helpful for inexperienced users in kinetic modeling. In contrast to Encora, ModelMaker is quite user-friendly and calculates standard deviations of the estimates. On the other hand, the kinetic model has to be implemented by the user in a mixed graphic and equation-oriented way.

Among the computer programs tested gPROMS is the most sophisticated one. Even though the user has to implement the kinetic model equations manually, which can be difficult for non-specialists, gPROMS outperforms the other programs in terms of versatility like the possibility to implement partial differential equations required for spatially distributed systems as exemplified in Spiess et al. (2008b). The most important advantage is the detailed statistical analysis which is provided along with the parameter estimates. This contains not only standard deviations, but also confidence intervals, residual charts, the variancecovariance matrix, and the correlation matrix. Residual charts support the detection of systematic deviations between experimental data and model prediction (Bard, 1974; CornishBowden, 2001). From the variance-covariance matrix confidence ellipsoids can be calculated (Bard, 1974; Rakels et al., 1994), as shown in Figure 2-5.
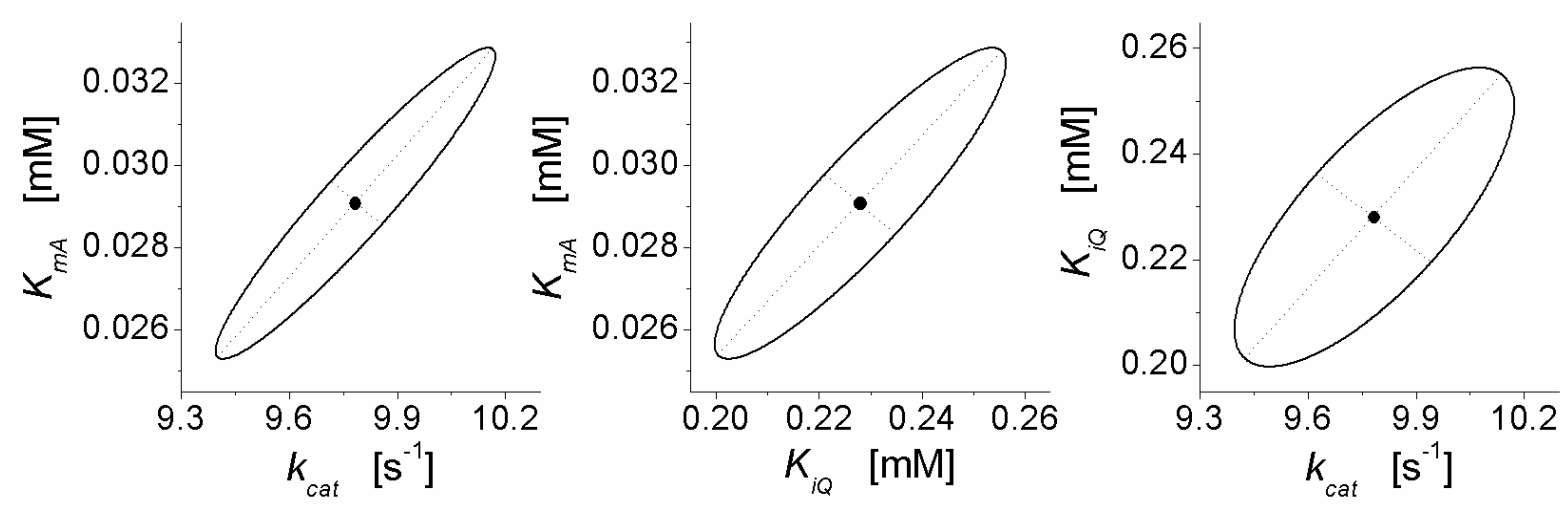

Figure 2-5: Confidence ellipsoids of the kinetic parameters obtained from gPROMS.

Figure 2-5 demonstrates that all kinetic parameters are correlated since the major axes of the ellipsoids are not parallel to the coordinate axes. The correlation is positive, which means that a too high estimate of one parameter will cause a too high estimate of the other parameter. The same is true for too low estimates (Franceschini and Macchietto, 2008c). These correlations are also visible in Figure 2-4, where too high estimates for $k_{c a t}$ mostly come along with too high estimates for $K_{i Q}$ and $K_{m A}$ and vice versa. For this reason, optimal experiments should be designed in order to decrease this correlation and to improve the statistical significance of the parameter estimates (Duggleby and Clarke, 1991; Lindner and Hitzmann, 
2006; Marquardt, 2005). This can e.g. be done with the optimal experimental design feature of gPROMS. This makes gPROMS the computer program of choice for the model-based experimental analysis (MEXA) of enzyme kinetics.

\subsubsection{Conclusions}

Progress curve analysis of enzymatic reactions can be performed using either algebraic or dynamic parameter estimation. If algebraic parameter estimation is applied, the concentration data have to be differentiated to get reaction rates. It is demonstrated that adequate regularization of the concentration data is essential prior to differentiation. A fast and simple regularization method is the use of smoothing splines. Applying this method, the accuracy of the parameter estimates obtained with MS-Excel or Origin is comparable to the estimates using dynamic parameter estimation. This is especially true if a large amount of data is available as typical for spectroscopic measurements. Among the computer programs which use dynamic parameter estimation (Encora, ModelMaker, and gPROMS), large differences of the estimated parameter values can occur. It becomes evident that deviations among published estimates for enzyme kinetic parameters can be caused by using different computer programs. For this reason, the applied computer program should always be specified. In general, gPROMS provides the most accurate parameter estimates together with a detailed statistical analysis. Therefore, gPROMS is chosen from the tested programs and applied for the subsequent progress curve analysis of enzymatic reactions.

\subsection{Mechanistic kinetic model for benzaldehyde lyase}

\subsubsection{Introduction}

Thiamin diphosphate (ThDP)-dependent enzymes, such as benzaldehyde lyase (BAL, EC 4.1.2.38), benzoylformate decarboxylase (BFD, EC 4.1.1.7) and pyruvate decarboxylase (PDC, EC 4.1.1.1), catalyze carboligase reactions with excellent enantioselectivity (Demir et al., 2001b; Iding et al., 2000; Pohl et al., 2002). This formation of carbon-carbon bonds constitutes one of the key transformations in synthetic organic chemistry (Faber and Kroutil, 2005; Woodley, 2008). Especially the production of chiral hydroxy ketones is of commercial interest since they are important building blocks of drugs and natural products (Demir et al., 
2001a). Since chemical methods involve complex reagents, auxiliaries, expensive catalysts, and protective groups to prevent the formation of unwanted side products, enzymatic synthesis routes are very promising (Schoemaker et al., 2003).

A reaction mechanism for the decarboxylation has been proposed for PDC (Kluger, 1987) and BFD (Weiss et al., 1988; Reynolds et al., 1988; Iding et al., 2000). According to these investigations, the reaction is catalyzed directly at the cofactor ThDP with an enaminecarbanion as intermediate. A similar mechanism with corresponding ThDP intermediates was later proposed for the carboligation using BAL (Demir et al., 2001b). It is assumed that the enzymes act only as stabilizers of the zwitter-ionic state of the intermediate (Jordan, 2003). Using isoelectric analogues of ThDP, it has been confirmed that the cofactor plays the crucial role in the reaction mechanism (Leeper et al., 2005). Moreover, X-ray diffraction analysis has revealed that among these enzymes, their cofactor orientation is remarkably similar (Mosbacher et al., 2005). To optimize processes using ThDP-dependent enzymes, it is essential to derive a kinetic model that describes the kinetics of these enzymes in a mechanistically correct manner. According to Vasic-Racki et al. (2003) such a model contributes to an increase in knowledge about the process, which helps to identify optimal operating conditions. Most ThDP-dependent enzymes follow a similar mechanism. Among this group of enzymes, BAL is of special interest. BAL is a very active catalyst that is able to form and also to cleave chiral hydroxy ketones. Other ThDP-dependent enzymes, such as BFD, are not able to perform this cleavage due to steric hindrance (Knoll et al., 2006). Therefore, in order to fit the derived kinetic model, BAL is chosen.

BAL was discovered by Gonzalez and Vicuna (1989), who had previously observed that Pseudomonas fluorescens is able to grow on media with benzoin or anisoin as the only carbon source (Gonzalez et al., 1988). BAL is a homo-tetramer of four identical subunits of 563 amino acid residues, corresponding to a molecular mass of 58,919 Da. The cofactors ThDP and $\mathrm{Mg}^{2+}$ are bound at the interface of a dimer, such that one binding site is formed by two monomers. Nevertheless, the tetrameric enzyme contains four active centers (Mosbacher et al., 2005). BAL has a broad substrate spectrum of differently substituted benzaldehydes. According to Dünkelmann et al. (2002) one substrate molecule acts as donor whereas another acts as acceptor. 
To date, this donor-acceptor principle has been applied only for the synthesis with two different substrates, namely $A$ and $B$. By varying the concentration of $A$, while $B$ is in surplus, and vice-versa, the Michaelis constants, $K_{m A}$ and $K_{m B}$, respectively, can be determined by initial rate measurements. However, the special situation of the synthesis with two identical substrate molecules $A$ to a symmetric benzoin renders this approach infeasible. Therefore, the occurrence of different $K_{m}$-values, for both the donor and the acceptor, has been ignored in previous studies that assume that only a single $K_{m}$-value exists (e.g. Stillger et al., 2006; Hildebrand et al., 2007). Motivated by this lack of argument, a novel mechanistic kinetic model with two independent $K_{m}$-values is derived for the first time.

To fit this mechanistic kinetic model, progress curve analysis is performed, where concentrations are monitored for longer time periods, at most until the thermodynamic equilibrium is reached. Progress curve analysis should be preferred since it can provide more information than initial rate analysis (Duggleby, 2001). For example, effects occurring after a longer period of time, such as enzyme deactivation, can only be detected this way. Moreover, this approach is of industrial interest since for technical applications enzymatic reactions are used for much longer time periods until high conversions are reached. As shown in the previous Section 2.1, progress curve analysis requires special software for either algebraic or dynamic parameter estimation. Although such software is readily available nowadays, this approach is hardly being used in the biocatalytic community. One reason might be that experimenters are usually not much familiar with regularization techniques required for algebraic parameter estimation. If instead dynamic parameter estimation is applied, it is difficult to estimate parameters of non-linear models, if the quality of the initial guess is poor. To overcome this difficulty, Vasic-Racki et al. (2003) proposed to conduct first initial rate measurements to get reasonable initial guesses for the kinetic parameters. However, initial rate measurements are laborious and not feasible in the case of identical substrates. For this reason, the new mechanistic kinetic model for the condensation reaction of two identical substrates with BAL was fitted directly to progress curves without preceding initial rate measurements. The predictability of the derived model was analyzed using advanced statistics tools. Optimal experiments were designed to estimate the parameters precisely. Moreover, the effects of reasonable assumptions on the model predictability have been checked. 


\subsubsection{Materials and methods}

\subsubsection{Experimental}

All chemical substances were of analytical grade and purchased by Sigma-Aldrich (Deisenhofen, Germany). 3,5-dimethoxy-benzaldehyde (DMBA) was used as substrate yielding the product $(R)-3,3$ ',5,5'-tetramethoxy-benzoin (TMB). BAL was fermented in Escherichia coli as a fusion protein with His-tag and purified with affinity chromatography. The enzyme was stored as a lyophilisate. The applied assay contained $50 \mathrm{mM}$ $\mathrm{KH}_{2} \mathrm{PO}_{4} / \mathrm{K}_{2} \mathrm{HPO}_{4}$ buffer and both cofactors, $0.25 \mathrm{mM} \mathrm{MgCl}$ and $0.25 \mathrm{mM} \mathrm{ThDP}$. To increase the solubility of the aromatic compounds, $30 \%(\mathrm{v} / \mathrm{v})$ of the cosolvent dimethylsulfoxide (DMSO) was added, which also has a stabilizing effect on the enzyme (Dominguez de Maria et al., 2006). Finally, the $\mathrm{pH}$ value was adjusted to 8.5 and the ionic strength to $150 \mathrm{mM}$. The $\mathrm{pH}$ value is one unit lower than the activity optimum of 9.5 observed by Dominguez de Maria et al. (2006), but leads to a higher stability of the enzyme. Consequently, the $\mathrm{pH}$ value of 8.5 was chosen as a compromise between stability and activity.

Since BAL (Pseudomonas fluorescens Biovar I) is a tetramer of four identical subunits, the activity is related to one subunit. Assuming pure enzyme without additional proteins, the molar concentration of the subunit is calculated by dividing the weighted lyophilisate by the molecular weight $(59,800 \mathrm{Da})$ of the subunit including the His-tag (Janzen et al., 2006).

The reaction kinetic measurements were conducted at $25^{\circ} \mathrm{C}$ using a fluorimeter (LS55, PerkinElmer, Waltheim, USA). The concentration of the substrate DMBA was monitored by exciting at $360 \mathrm{~nm}$ and recording the fluorescence intensity at $470 \mathrm{~nm}$. Nine progress curves were measured (Table 2-4). The initial substrate concentration was varied between 1.5 and $3 \mathrm{mM}$. Owing to the limited solubility of the product, the initial substrate concentration could not exceed $3 \mathrm{mM}$. Otherwise, the concentration of the formed product would have exceeded $1 \mathrm{mM}$, where the product starts to precipitate. The enzyme concentrations have been chosen sufficiently high to prevent enzyme deactivation before equilibrium was reached. This effect has been investigated earlier (data not shown). As long as no significant enzyme deactivation or degradation of reactants occurs, the enzyme concentration can be considered to be constant in each single dynamic simulation (Selwyn, 1965). Therefore, two different enzyme 
concentrations are sufficient for the task of model identification. The measurements have been performed until equilibrium - indicated by a constant fluorescence signal - was reached.

Table 2-4: $\quad$ Initial experimental conditions.

\begin{tabular}{lllll}
\hline Experiment & $c_{A O}[\mathrm{mM}]$ & $c_{P O}[\mathrm{mM}]$ & $c_{E}[\mathrm{mM}]$ & $t_{\text {exp }}[\mathrm{s}]$ \\
\hline A & 3.00 & 0 & $8.33 \cdot 10^{-5}$ & 1500 \\
B & 2.75 & 0 & $8.33 \cdot 10^{-5}$ & 1500 \\
C & 2.50 & 0 & $8.33 \cdot 10^{-5}$ & 1500 \\
D & 2.25 & 0 & $8.33 \cdot 10^{-5}$ & 1500 \\
E & 2.00 & 0 & $8.33 \cdot 10^{-5}$ & 700 \\
F & 1.50 & 0 & $8.33 \cdot 10^{-5}$ & 1000 \\
G & 3.00 & 0 & $4.17 \cdot 10^{-5}$ & 3000 \\
H & 2.75 & 0 & $4.17 \cdot 10^{-5}$ & 3000 \\
I & 2.50 & 0 & $4.17 \cdot 10^{-5}$ & 3000 \\
\hline
\end{tabular}

\subsubsection{Model identification approach}

The procedure applied for this study is illustrated in Figure 2-6. It follows the model-based experimental analysis (MEXA) approach described by Marquardt (2005). Based on initial knowledge about the enzyme mechanism, a kinetic model is developed (1), which takes all micro-reaction steps into account. This model is fitted to experimental data (2) and the quality of the estimated parameters is statistically evaluated in terms of the standard deviations of the parameters and the correlation matrix (Bard, 1974) (3). Moreover, confidence ellipsoids can be analyzed, which visualize the correlation between a pair of parameters (Franceschini and Macchietto, 2007, 2008a, 2008b, 2008c) and residuals can be plotted, which help to detect anomalies not predicted by the kinetic model (Bard, 1974; Cornish-Bowden, 2001). To detect the sensitivities of the model parameters, sensitivity analyses are performed.

If a kinetic model is not able to predict the experimental data satisfactorily, the model has to be revised in order to include more or other phenomena (Marquardt, 2005) (4). However, in many cases, the model can predict the experimental data quite well, but the parameters cannot be estimated with sufficient precision. In general, there are two possible reasons for this. First the experimental data can be insufficient for estimating the parameters precisely. This is 
especially the case if the experimentally accessible region is rather limited, as e.g. due to low solubilities of the reactants. In this case, the model can, in principle, be identified by expanding the experimental limitations. Model-based optimal experimental design techniques (5) and in silico experiments (6) can be used to identify the necessary experimental region (Walter and Pronzato, 1990). The second reason for unsatisfactory parameter estimates is a model being too complex. The model is then over-specified. In this case, the model parameters are never identifiable, even after conducting optimal in silico experiments. In this case, the model has to be revised in order to reduce the model complexity (4).

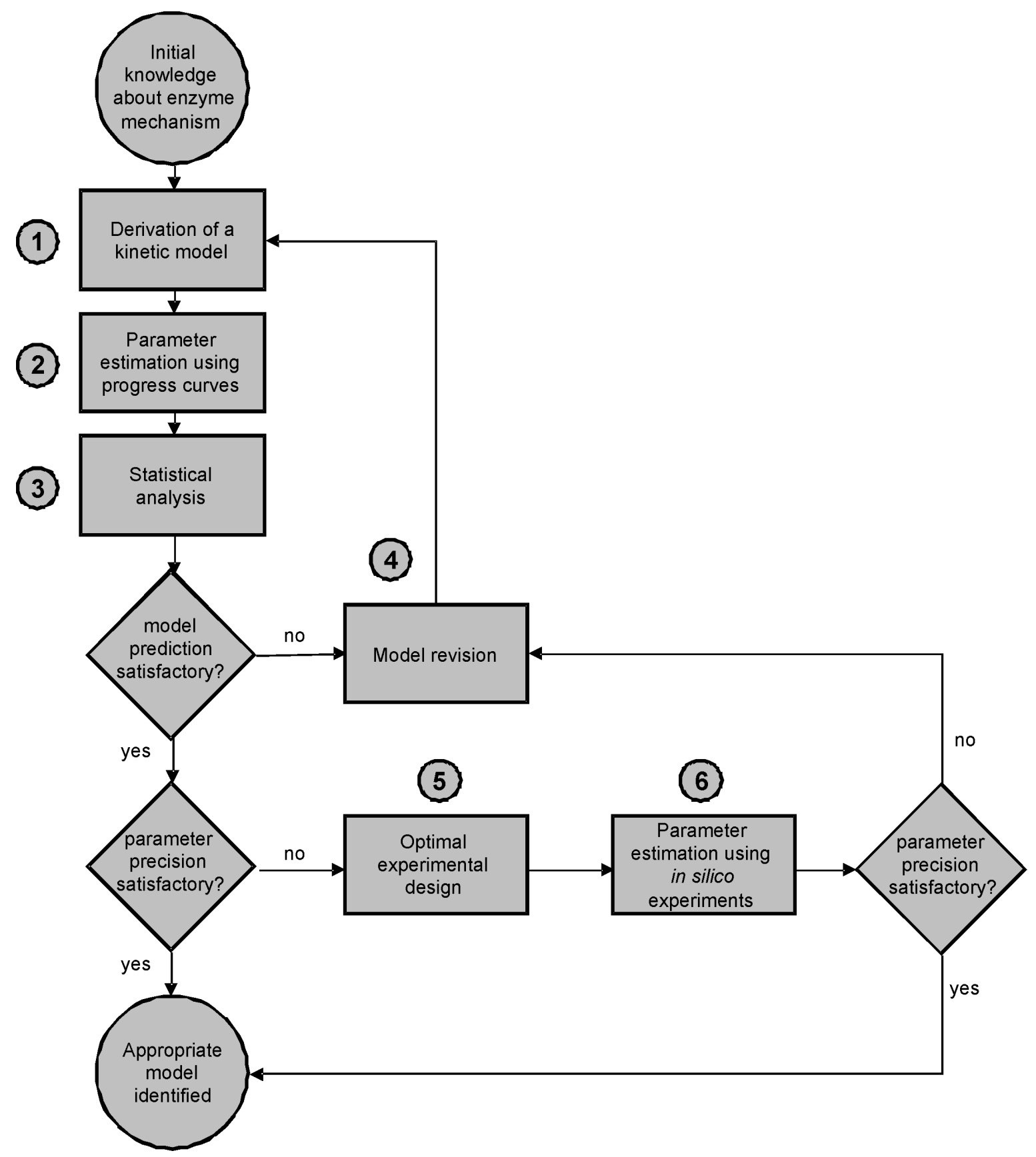

Figure 2-6: $\quad$ Applied procedure for deriving the kinetic model. 
Since the software package gPROMS (version 3.0.2, Process System Enterprise Ltd., London, UK) turned out to be the most accurate and reliable analysis software (Section 2.1), it was applied for the purposes of modeling, simulations, and dynamic parameter estimation. The addition of Gaussian noise for the in silico experiments and the sensitivity analysis were carried out using Matlab (version 7.3, The MathWorks Inc., Natick, USA).

\subsubsection{Results and discussion}

The model reaction for the derivation and experimental verification of the kinetic model for BAL is the carboligation of 3,5-dimethoxy-benzaldehyde (DMBA) to (R)-3,3',5,5'tetramethoxy-benzoin (TMB) (Figure 2-7A). Initial experiments have shown that the substrate DMBA, which is substituted in meta-position, can act both as acceptor and donor forming the symmetric TMB.

\subsubsection{Derivation of the mechanistic kinetic model}

The mechanism proposed by Demir et al. (2001b) was adapted for the synthesis of TMB. In Figure 2-7B, the three main micro-reaction steps for constructing the kinetic model are illustrated. The isomerization and proton transfer steps are assumed to be very fast and thus not rate-limiting. The micro-reaction rate constants $k_{i}$ denote the rate constants for the microreaction steps. All these micro-reactions are reversible. Since two substrate molecules form one product molecule, the investigated reaction is denoted to follow an ordered bi-uni reaction mechanism (Cleland, 1963). This can be written in Cleland's notation as depicted in Figure 2-7C. To distinguish between the first and the second binding substrate, they are denoted with $A$ and $B$, respectively. $E$ denotes the enzyme with the bound cofactors ThDP and $\mathrm{Mg}^{2+}$. As these cofactors are bound to the enzyme, the complex of free enzyme and cofactors can be treated as one single species. In the first reaction, the substrate $A$ binds to the enzyme $E$ forming the enzyme-substrate complex $E A$. After this, $B$ binds to $E A$ forming the ternary $E A B$ complex. The enzyme is then recovered by forming the product $P$. 


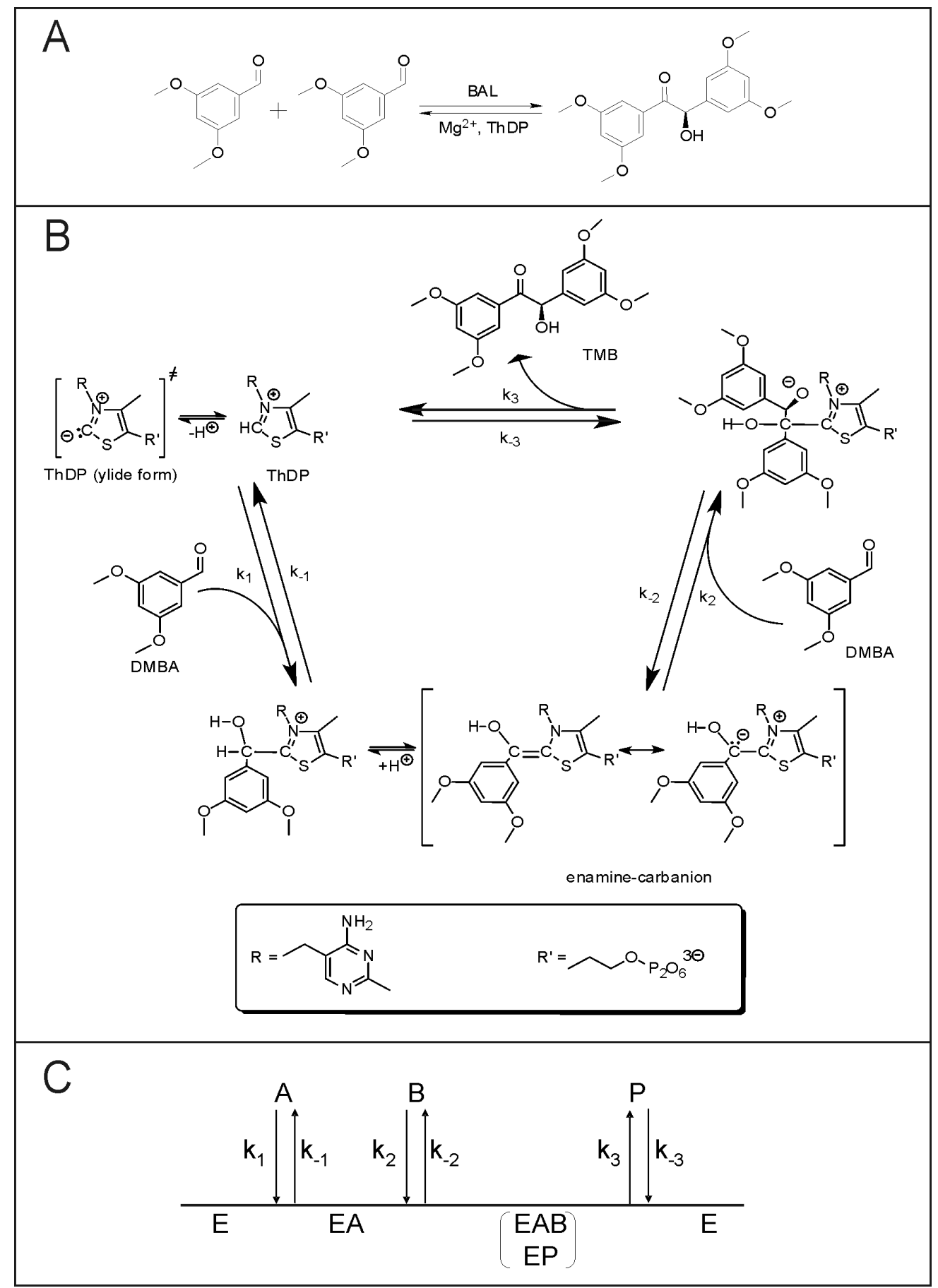

Figure 2-7: $\quad$ Synthesis reaction of 3,5-dimethoxy-benzaldehyde (DMBA) to $(R)$-3,3',5,5'-tetramethoxybenzoin (TMB) (A). Reaction mechanism of BAL for the TMB synthesis adapted from Demir et al. (2001b) (B). Reaction mechanism of a bi-uni-reaction according to Cleland (1963) (C).

Applying the method of King and Altman (1956) Eq. 2-4 was derived, where $c_{i}$ denotes the concentration of species $i$. This rate equation is mechanistically correct since it takes all micro-reaction steps into account including the inevitable competitive inhibitions of the forward reaction by $P$ and of the reverse reaction by $A$. Moreover, the reverse reaction is considered. Consequently, this rate law is valid for ordered bi-uni reactions under all 
concentrations of the reactants. Therefore, it should be preferred over multiplying simple Michaelis-Menten equations for all substances as it was done in previous publications (e.g. Stillger et al., 2006; Hildebrand et al., 2007).

$$
v=-\frac{d c_{A}}{d t}=-\frac{d c_{B}}{d t}=\frac{d c_{P}}{d t}=\frac{\frac{K_{\text {catf }}}{K_{i A} K_{m B}}\left(c_{A} \cdot c_{B}-\frac{c_{P}}{K_{e q}}\right)}{1+\frac{c_{A}}{K_{i A}}+\frac{K_{m A} \cdot c_{B}}{K_{i A} \cdot K_{m B}}+\frac{c_{A} \cdot c_{B}}{K_{i A} \cdot K_{m B}}+\frac{c_{P}}{K_{m P}}+\frac{c_{B} \cdot c_{P}}{K_{m P} \cdot K_{i B}}} \cdot c_{E}
$$

Eq. 2-4 shows that the constructed model contains seven parameters, whereas only six microreaction rate constants exist. This means that one parameter is dependent on the others. To detect this redundancy, the method of Straathof and Heijnen (1996) was applied and thus Eq. 2-5 was identified. Table 2-5 lists the definitions of the remaining independent parameters.

$$
K_{i B}=\frac{K_{m B} \cdot K_{i A}}{K_{m A} \cdot\left[1-\left(\frac{K_{m A}}{K_{i A}}-1\right) \cdot \frac{K_{m P}}{K_{e q} \cdot K_{m B} \cdot K_{i A}}\right]}
$$

Table 2-5: Definitions of the model parameters for the mechanistic kinetic model.

\begin{tabular}{llll}
\hline Parameter & Definition & Biological interpretation & Unit \\
\hline$k_{\text {catf }}$ & $k_{3}$ & Maximum turnover number & $\mathrm{s}^{-1}$ \\
$K_{\text {eq }}$ & $\frac{k_{1} \cdot k_{2} \cdot k_{3}}{k_{-1} \cdot k_{-2} \cdot k_{-3}}$ & Equilibrium constant & $\mathrm{mM}^{-1}$ \\
$K_{m A}$ & $\frac{k_{3}}{k_{1}}$ & Affinity constant of $A$ to $E$ & $\mathrm{mM}$ \\
$K_{m B}$ & $\frac{k_{-2}+k_{3}}{k_{2}}$ & Affinity constant of $B$ to $E A$ & $\mathrm{mM}$ \\
$K_{m P}$ & $\frac{k_{-1} \cdot\left(k_{-2}+k_{3}\right)}{k_{-3} \cdot\left(k_{-1}+k_{-2}\right)}$ & Affinity constant of $P$ to $E$ & $\mathrm{mM}$ \\
$K_{i A}$ & $\frac{k_{-1}}{k_{1}}$ & Dissociation constant of $E A$ & $\mathrm{mM}$ \\
\hline
\end{tabular}


Besides $K_{i B}$ there are two more dependent kinetic parameters, $k_{c a t r}$ and $K_{i P}$, which are functions of the independent parameters:

$$
\begin{gathered}
k_{\text {catr }}=\frac{k_{-1} \cdot k_{-2}}{k_{-1}+k_{-2}}=\frac{k_{\text {catf }} \cdot K_{m P}}{K_{m B} \cdot K_{i A} \cdot K_{e q}} \\
K_{i P}=\frac{k_{3}}{K_{-3}}=\frac{K_{m P}}{1-\left(\frac{K_{m A}}{K_{i A}}-1\right) \cdot \frac{K_{m P}}{K_{e q} \cdot K_{m B} \cdot K_{i A}}}
\end{gathered}
$$

These two additional kinetic parameters do not occur in the kinetic model (Eqs. 2-4-2-5), but have a physical meaning $-k_{\text {catr }}$ is the maximal turnover number for the reverse reaction and $K_{i P}$ is the product inhibition constant. With the definitions of the model parameters (Table 2-5), the micro-reaction rate constants can be calculated:

$$
\begin{gathered}
k_{1}=\frac{k_{\text {catf }}}{K_{m A}} \\
k_{-1}=\frac{K_{i A} \cdot k_{\text {catf }}}{K_{m A}} \\
k_{2}=\frac{k_{\text {catf }} \cdot\left(K_{m P} \cdot K_{i A}-K_{m P} \cdot K_{m A}+K_{i A}{ }^{2} \cdot K_{m B} \cdot K_{e q}\right)}{K_{m B} \cdot\left(K_{i A}{ }^{2} \cdot K_{m B} \cdot K_{e q}-K_{m P} \cdot K_{m A}\right)} \\
k_{-2}=\frac{K_{m P} \cdot K_{i A} \cdot K_{c a t f}}{K_{i A}{ }^{2} \cdot K_{m B} \cdot K_{e q}-K_{m P} \cdot K_{m A}} \\
k_{-3}=\frac{k_{c a t f} \cdot\left(K_{m P} \cdot K_{i A}-K_{m P} \cdot K_{m A}+K_{i A}{ }^{2} \cdot K_{m B} \cdot K_{e q}\right)}{K_{m P} \cdot K_{i A}{ }^{2} \cdot K_{m B} \cdot K_{e q}}
\end{gathered}
$$

In the investigated special case of two identical substrates, the concentration of $A$ and $\mathrm{B}$ are always equal $\left(c_{A}=c_{B}\right)$. So Eq. 2-4 is transformed to Eq. 2-14: 


$$
v=-\frac{1}{2} \cdot \frac{d c_{A}}{d t}=\frac{d c_{P}}{d t}=\frac{\frac{k_{c a t f}}{K_{i A} \cdot K_{m B}} \cdot\left(c_{A}{ }^{2}-\frac{c_{P}}{K_{e q}}\right)}{1+\frac{c_{A}}{K_{i A}} \cdot\left(1+\frac{K_{m A}}{K_{m B}}\right)+\frac{c_{A}{ }^{2}}{K_{i A} \cdot K_{m B}}+\frac{c_{P}}{K_{m P}}+\frac{c_{A} \cdot c_{P}}{K_{m P} \cdot K_{i B}}} \cdot c_{E}
$$

The thermodynamic equilibrium constant for the symmetric carboligation is defined as follows:

$$
K_{e q}=\frac{c_{P}(t \rightarrow \infty)}{c_{A}(t \rightarrow \infty)^{2}}
$$

If, in contrast, the reaction is modeled as uni-uni reaction as done by e.g. Stillger et al. (2006), the thermodynamic equilibrium constant becomes:

$$
K_{e q}=\frac{c_{P}(t \rightarrow \infty)}{c_{A}(t \rightarrow \infty)}
$$

As a result, uni-uni models predict a wrong thermodynamic equilibrium, if different substrate concentrations are used. Thus, they can only be used for modeling initial rates and should not be used for processes, in which high conversions are intended.

\subsubsection{Parameter estimation}

Nine experiments with varying experimental conditions according to Table 2-4 have been conducted. The experimental data of these experiments have been used to estimate the parameters of the model given in Eqs. 2-4-2-5. After the parameter estimation step, the model is able to reproduce the experimental data accurately (Figure 2-8). 


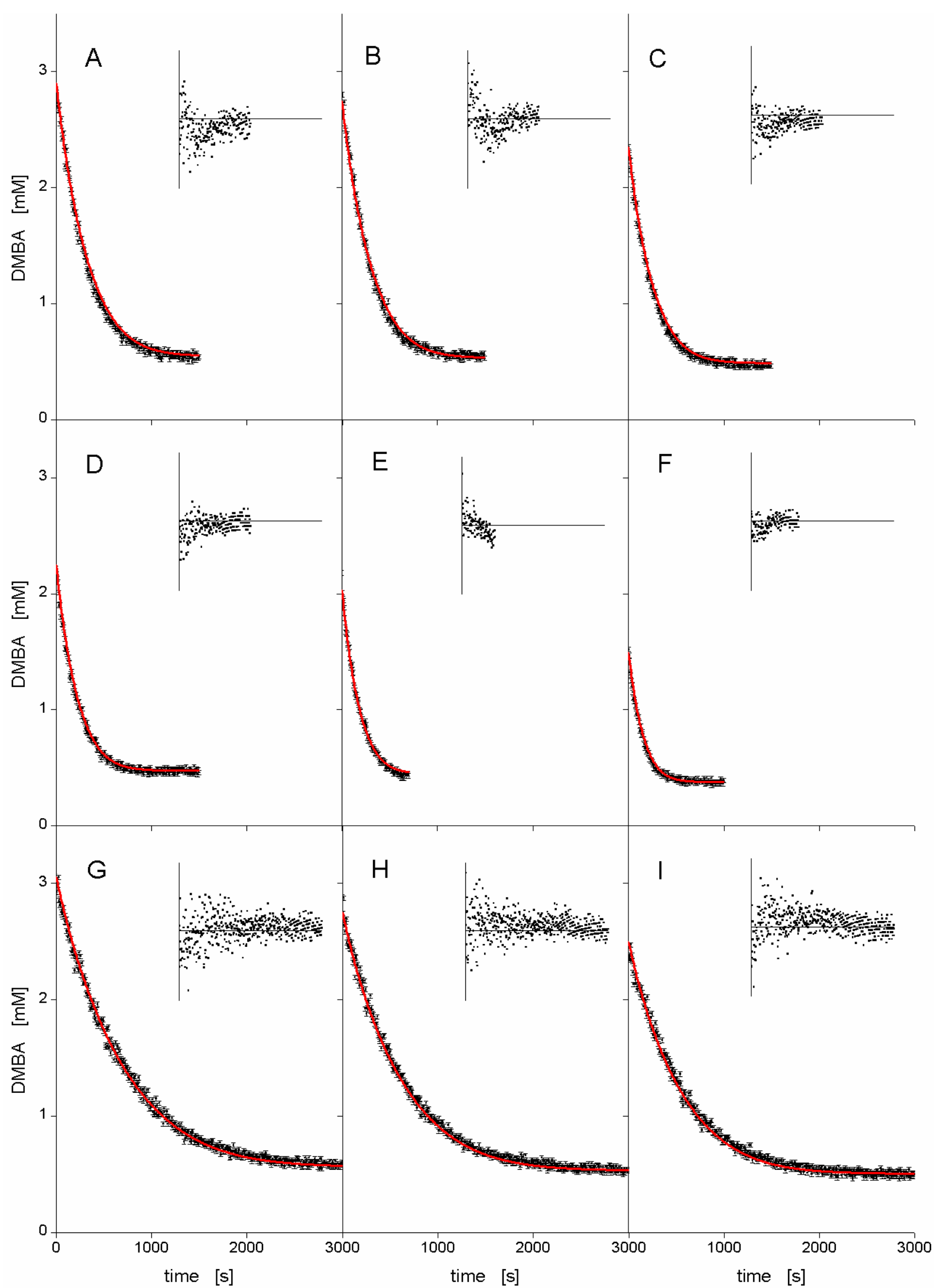

Figure 2-8: Comparison of the fitted mechanistic kinetic model with the experimental data using experiments A-I (Table 2-4). The residuals are shown as small inlets. 
Table 2-6: $\quad$ Parameter estimates and standard deviations using the derived mechanistic kinetic model.

\begin{tabular}{lll}
\hline Parameter & Unit & Estimate \\
\hline$k_{\text {catf }}$ & $\mathrm{s}^{-1}$ & $46.123 \pm 0.991$ \\
$K_{\text {eq }}$ & $\mathrm{mM}^{-1}$ & $3.934 \pm 0.017$ \\
$K_{m A}$ & $\mathrm{mM}$ & $0.050 \pm 0.426$ \\
$K_{m B}$ & $\mathrm{mM}$ & $0.005 \pm 0.503$ \\
$K_{m P}$ & $\mathrm{mM}$ & $0.975 \pm 28.323$ \\
$K_{i A}$ & $\mathrm{mM}$ & $0.990 \pm 121.167$ \\
\hline
\end{tabular}

However, the precision of the parameter estimates is quite diverse (Table 2-6). The thermodynamic equilibrium constant $K_{e q}$ can be estimated very precisely. The precision of the maximum turnover number $k_{\text {catf }}$ is also acceptable. All other kinetic parameters have unacceptably high imprecisions since the standard deviations are much larger than the estimated values. Hence, these parameters cannot be considered to be identifiable under the present experimental conditions. This hypothesis was strengthened by the obtained correlation matrix (Table 2-7).

Table 2-7: Correlation matrix of the estimated parameters using the derived mechanistic kinetic model.

\begin{tabular}{|c|c|c|c|c|c|c|}
\hline & $k_{\text {catf }}$ & $K_{e q}$ & $K_{i A}$ & $K_{m A}$ & $K_{m B}$ & $K_{m P}$ \\
\hline$k_{\text {catf }}$ & 1.00 & -0.13 & -0.86 & -0.83 & 0.86 & -0.64 \\
\hline$K_{e q}$ & & 1.00 & -0.10 & -0.12 & 0.10 & -0.34 \\
\hline$K_{i A}$ & & & 1.00 & 1.00 & -1.00 & 0.93 \\
\hline$K_{m A}$ & & & & 1.00 & -1.00 & 0.94 \\
\hline$K_{m B}$ & & & & & 1.00 & -0.93 \\
\hline$K_{m P}$ & & & & & & 1.00 \\
\hline
\end{tabular}

Most kinetic parameters are highly correlated and, therefore, cannot be estimated independently. To illustrate the correlations between two parameters, confidence ellipsoids were drawn (Figure 2-9). The semi-axes of the ellipsoid for the parameters $K_{e q}$ and $k_{\text {catf }}$ are almost parallel to the coordinate axes, which indicates a low correlation of both parameters. In contrast to this, the confidence ellipsoid of $K_{m P}$ and $K_{i A}$ implies a strong positive correlation of these two parameters. Thus, they cannot be estimated independently from each other. Moreover, the region of the $95 \%$ confidence ellipsoid of $K_{m P}$ and $K_{i A}$ also includes negative 
values for the kinetic parameters. This highlights the unreliability of these estimates since only positive kinetic parameters are possible.
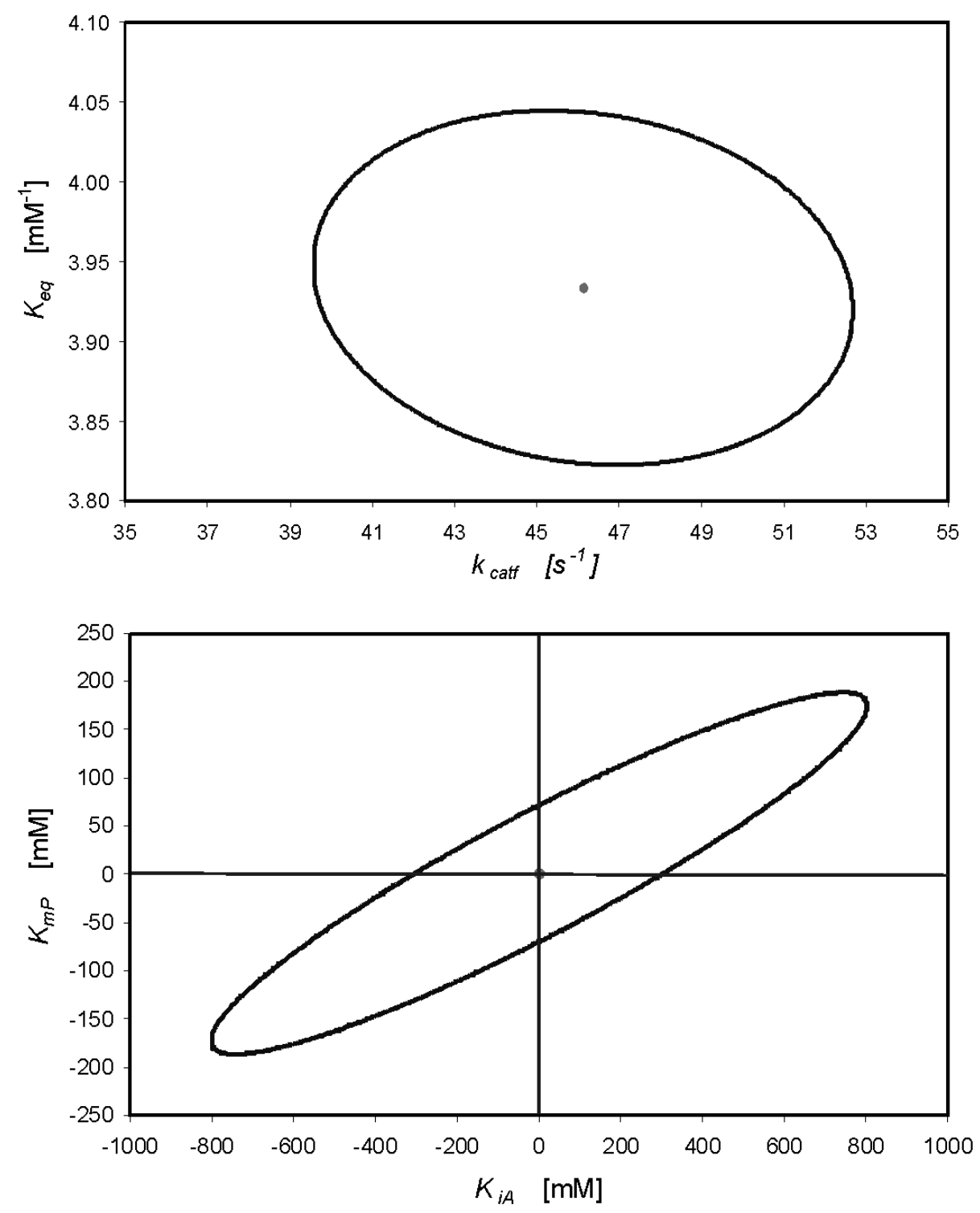

Figure 2-9: $\quad 95 \%$ confidence ellipsoids for $K_{\text {eq }} / k_{\text {catf }}$ (top) and $K_{m P} / K_{\text {iA }}$ (bottom).

According to the procedure described in Section 2.2.2.2, two possible reasons for unidentifiable parameters exist. The experimental data might contain insufficient information or the model structure might be too complex. To check whether this poor parameter precision is due to the tight experimental limitations, eight optimal experiments were designed. The degrees of freedom for the optimal experimental design were the initial concentrations of the substrate and the product and the experiment duration. The E-optimality criterion was used as the objective function (Walter and Pronzato, 1990). Reasonable limits have been used for all degrees of freedom. The aim was to determine whether the model parameters are generally 
not identifiable or whether they are identifiable under different experimental conditions, such as different substrate concentrations. Moreover, the addition of product at the beginning of the reaction was investigated, which was expected to cause a higher precision of the parameter estimates.

Table 2-8: Optimal experimental design for in silico experiments using the mechanistic kinetic model. For the optimal experimental design the degrees of freedom were the initial substrate concentration $c_{A O}$ (limits: $0-5 \mathrm{mM}$ ), the initial product concentration $c_{P 0}$ (limits: $0-5 \mathrm{mM}$ ) and the measurement duration $t$ (limits: 50-640 s). The enzyme concentration was held constant to $1.25 \cdot 10^{-4} \mathrm{mM}$.

\begin{tabular}{llll}
\hline Experiment & $c_{A O}[\mathrm{mM}]$ & $c_{P 0}[\mathrm{mM}]$ & $t[\mathrm{~s}]$ \\
\hline $\mathrm{J}$ & 0.585 & 0.000 & 360 \\
$\mathrm{~K}$ & 0.000 & 2.300 & 640 \\
$\mathrm{~L}$ & 0.585 & 0.000 & 360 \\
$\mathrm{M}$ & 0.900 & 0.050 & 360 \\
$\mathrm{~N}$ & 4.200 & 0.000 & 340 \\
$\mathrm{O}$ & 0.000 & 3.600 & 292 \\
$\mathrm{P}$ & 2.200 & 1.400 & 325 \\
$\mathrm{Q}$ & 0.050 & 0.100 & 50 \\
\hline
\end{tabular}

The designed optimal experiments are listed in Table 2-8. These experiments confirm the assumption that it is advantageous to perform also measurements with initial product concentrations. Interestingly, the designed experiments contain quite different time scales. An explanation for this could be that the longer lasting experiments are optimizing the estimation precision of the equilibrium constant $K_{\text {eq }}$, whereas the shorter ones focus on the kinetic parameters in the beginning of the experiment such as $k_{\text {catf }}$.

Since the experimental limits could not be expanded sufficiently, the optimal experiments were carried out in silico. Therefore, simulations with the fitted mechanistic kinetic model (Eqs. 2-5 and 2-14) and the conditions as determined by the optimal experiments were carried out. The simulated data for the substrate concentration were disturbed with Gaussian noise with a constant standard deviation of $0.001 \mathrm{mM}$. This low noise level, compared to the experimental error of about $0.05 \mathrm{mM}$, was used to assure that the experimental error was not the reason for a potential unidentifiability of the kinetic parameters. The obtained data were 
then treated as "real" experimental data for fitting the model. These data can be considered as taken under ideal conditions, which means that no solubility problems exist, enantiopure product is available and the measurement technique is highly precise.

Table 2-9: $\quad$ Parameter estimates and standard deviations after performing optimal in silico experiments using the mechanistic kinetic model.

\begin{tabular}{llr}
\hline Parameter & Unit & \multicolumn{1}{l}{ Estimate } \\
\hline$k_{\text {catf }}$ & $\mathrm{s}^{-1}$ & $46.153 \pm 0.024$ \\
$K_{\text {eq }}$ & $\mathrm{mM}^{-1}$ & $3.936 \pm 0.002$ \\
$K_{m A}$ & $\mathrm{mM}$ & $0.050 \pm 0.003$ \\
$K_{m B}$ & $\mathrm{mM}$ & $0.007 \pm 0.004$ \\
$K_{m P}$ & $\mathrm{mM}$ & $1.205 \pm 0.303$ \\
$K_{i A}$ & $\mathrm{mM}$ & $0.647 \pm 0.402$ \\
\hline
\end{tabular}

The obtained parameter estimates show much smaller standard deviations which indicates that the experimentally accessible region has indeed been too limited in the first set of experiments and that the measurement technique is not sufficiently precise (Table 2-9). The confidence ellipsoid for $K_{m P}$ and $K_{i A}$ (Figure 2-10) illustrates that the confidence region decreased remarkably compared to the previous confidence ellipsoid of these parameters depicted in Figure 2-9 (bottom). On the other hand, the confidence region for $K_{i A}$ still reaches negative values. Moreover, the correlation of $K_{m P}$ and $K_{i A}$ is still very high.

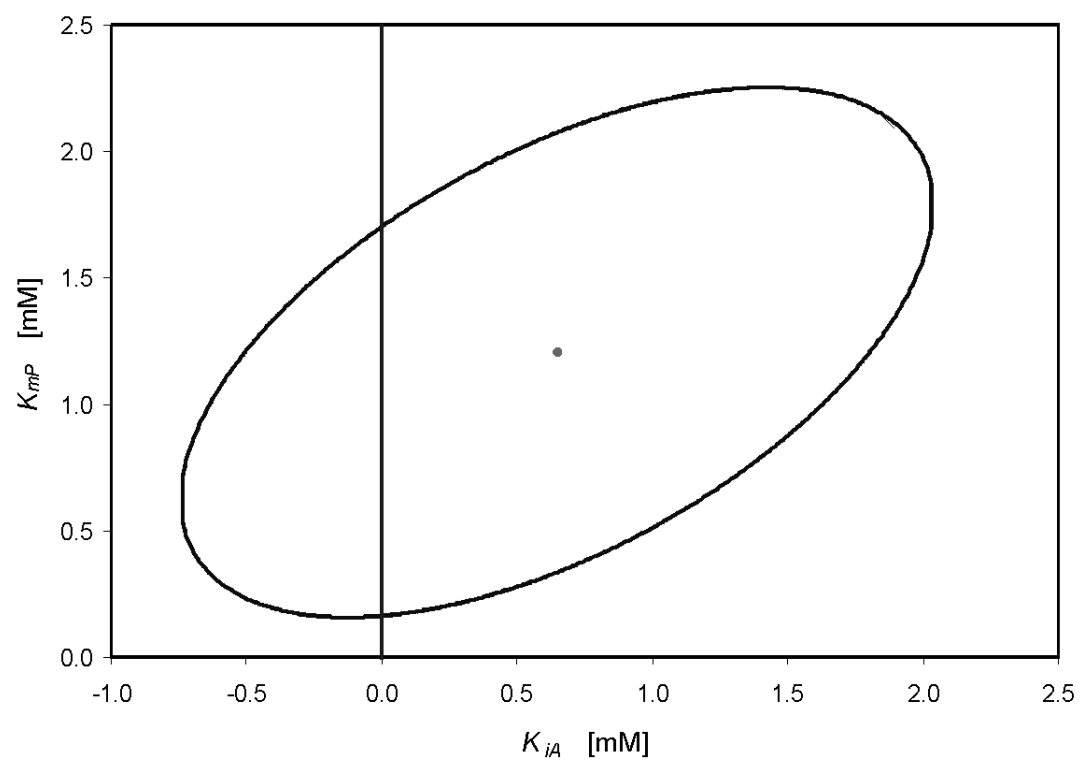

Figure 2-10: $\quad 95 \%$ confidence ellipsoid for $K_{m P} / K_{i A}$ after performing in silico experiments. 
High correlations between the kinetic parameters are also observed for other parameters (Table 2-10). Thus, even if the in silico experiments described above are used and the model is assumed to be correct some model parameters can still not be estimated with sufficient precision. Therefore, it can be concluded that the tight experimental conditions are not the main reason for the low parameter precision, but a too complex model with too many parameters.

Table 2-10: Correlation matrix of the estimated parameters after performing optimal in silico experiments using the mechanistic kinetic model.

\begin{tabular}{lcccccc}
\hline & $k_{\text {catf }}$ & $K_{\text {eq }}$ & $K_{i A}$ & $K_{m A}$ & $K_{m B}$ & $K_{m P}$ \\
\hline$k_{\text {catf }}$ & 1.00 & 0.15 & -0.63 & -0.40 & 0.64 & -0.03 \\
$K_{e q}$ & & 1.00 & -0.05 & 0.05 & 0.06 & 0.50 \\
$K_{i A}$ & & & 1.00 & 0.94 & -1.00 & 0.56 \\
$K_{m A}$ & & & & 1.00 & -0.93 & 0.64 \\
$K_{m B}$ & & & & & 1.00 & -0.55 \\
$K_{m P}$ & & & & & & 1.00 \\
\hline
\end{tabular}

Before revising the model, it is reasonable to investigate why these parameters cannot be identified. Thus, in order to detect how sensitive the parameters of the derived kinetic model are, a dynamic sensitivity analysis was performed. Figure 2-11 depicts the normalized sensitivities $S_{i, j}$ of the model parameters $\theta_{j}$ regarding the concentration $c_{i}$. These were obtained by using Eq. 2-17 and approximated with finite differences. The normalization makes the sensitivities dimensionless and considers differences in the order of magnitude between the parameters and between the concentrations. The obtained normalized sensitivities correspond to the percent change in the concentration caused by a one percent change in the value of the parameter. Different possibilities for normalization and scaling of sensitivities are found in Franceschini (2007).

$$
S_{i, j}=\frac{\theta_{j}}{c_{i}} \cdot \frac{\partial c_{i}}{\partial \theta_{j}}
$$




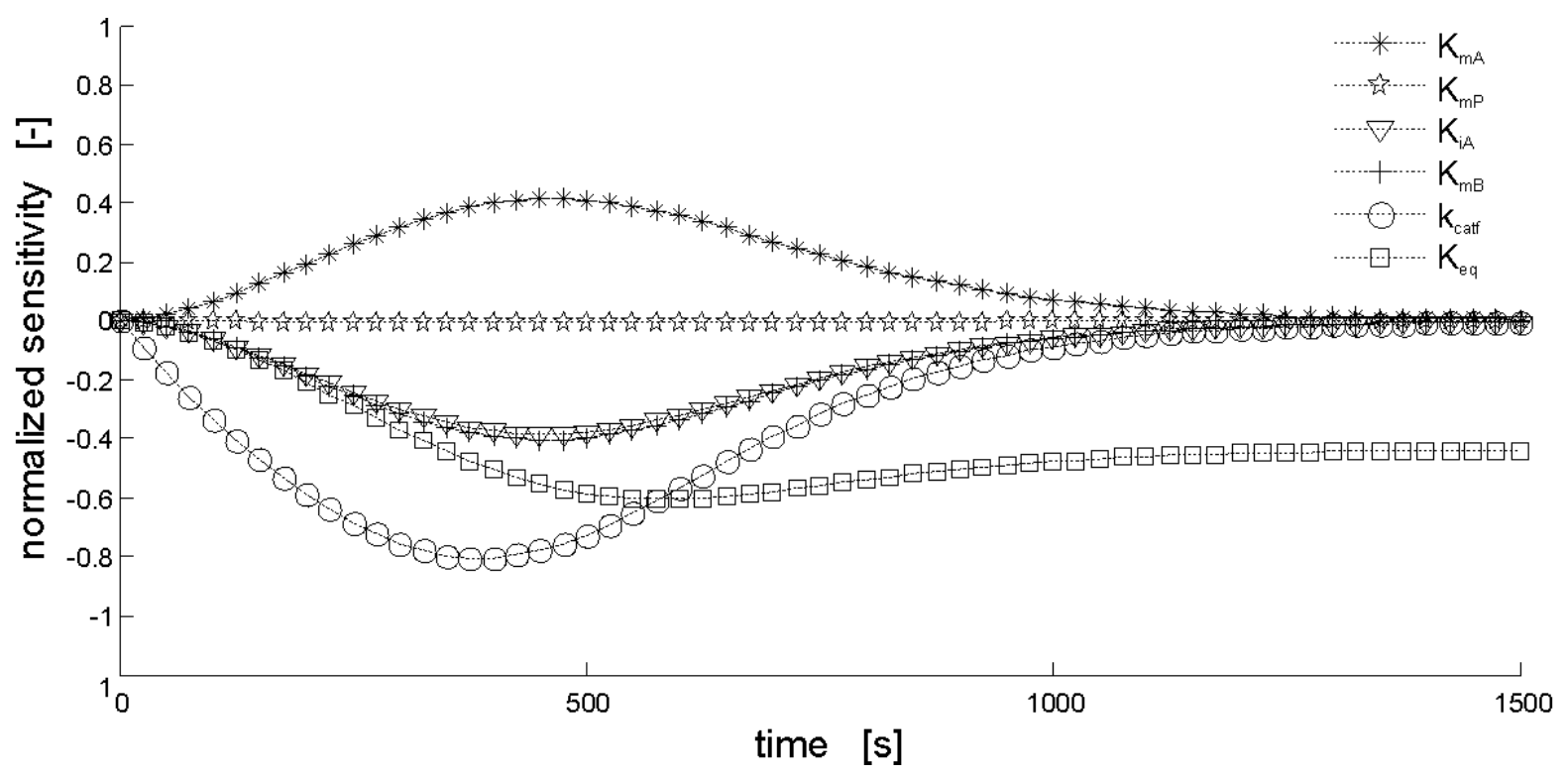

Figure 2-11: Normalized sensitivities of the model parameters of the mechanistic model regarding the substrate concentration using the initial conditions of experiment A (Table 2-4).

According to Figure 2-11, $k_{\text {catf }}$ is most sensitive at the start of the reaction and, therefore, can be estimated quite precisely. $K_{e q}$ is the only parameter that is sensitive for longer time periods. For this reason, its estimate is also very precise. $K_{m P}$ turns out to be very insensitive, which explains the high level of uncertainty for this parameter. The parameters $K_{i A}$ and $K_{m B}$ possess almost identical sensitivity curves. Therefore, they are strongly negatively correlated. The sensitivity curve of $K_{m A}$ is inverse to those of $K_{i A}$ and $K_{m B}$, which also causes high correlations to these parameters. Hence, these kinetic parameters cannot be estimated independently of each other. These findings show that the model comprises too many parameters and has to be revised according to the MEXA methodology.

\subsubsection{Model revision}

As shown in Figure 2-7B, the micro-reaction rate constants $k_{1}$ and $k_{2}$ denote the rate constants for the binding of the substrate molecules to the enzyme and the enzyme-substrate complex, respectively. These binding processes can be considered as a sum of three steps. First the substrate molecules diffuse from the bulk solution to the enzyme surface. Then the molecules diffuse to the cofactor within the active site. The last step is the binding of the molecules. The first substrate molecule binds to the ylide form of the cofactor and the second to the enaminecarbanion intermediate (Demir et al, 2001b; Figure 2-7B). Obviously, the only difference between the first and the second binding substrate is the binding to different forms of the cofactor. The first two diffusion steps are equally fast since the diffusion coefficients are the 
same. Assuming also that the binding step is equally fast, the micro-reaction rate constants $k_{1}$ and $k_{2}$ will have the same value. The same assumption is made for the micro-reaction rate constants $k_{-1}$ and $k_{-2}$ because in this case the same steps occur only in reverse order.

With this assumption only four independent micro-reaction rate constants remain without neglecting any reaction step. As a result, only four independent parameters can exist. Using the method of Straathof and Heijnen (1996), two more relations between the parameters could be identified (Eqs. 2-18 and 2-19), which are added to the kinetic model (Eqs. 2-5 and 2-14):

$$
\begin{gathered}
K_{m P}=\frac{K_{m B} \cdot\left(K_{m B}-K_{m A}\right)^{2} \cdot K_{e q}}{2 \cdot K_{m A}} \\
K_{i A}=K_{m B}-K_{m A}
\end{gathered}
$$

Consequently, only four independent parameters, which are listed in Table 2-11, have to be fitted during parameter estimation. It should be noted that the assumptions do not lead to the equality of $K_{m A}$ and $K_{m B}$.

Table 2-11: Definitions of the model parameters for the simplified kinetic model.

\begin{tabular}{llll}
\hline Parameter & Definition & Biological interpretation & Unit \\
\hline$k_{\text {catf }}$ & $k_{3}$ & Maximum turnover number & $\mathrm{s}^{-1}$ \\
$K_{\text {eq }}$ & $\frac{k_{1}{ }^{2} \cdot k_{3}}{k_{-1}{ }^{2} \cdot k_{-3}}$ & Equilibrium constant & $\mathrm{mM}^{-1}$ \\
$K_{m A}$ & $\frac{k_{3}}{k_{1}}$ & Affinity constant of $A$ to $E$ & $\mathrm{mM}$ \\
$K_{m B}$ & $\frac{k_{-1}+k_{3}}{k_{1}}$ & Affinity constant of $A$ to $E A$ & $\mathrm{mM}$ \\
\hline
\end{tabular}

The modified model has been fitted to the experimental data of the original experiments as described in Table 2-4. The experimental data together with the fitted progress curves and the residuals are provided in Figure 2-12. The estimated values are listed in Table 2-12 with their standard deviations. 


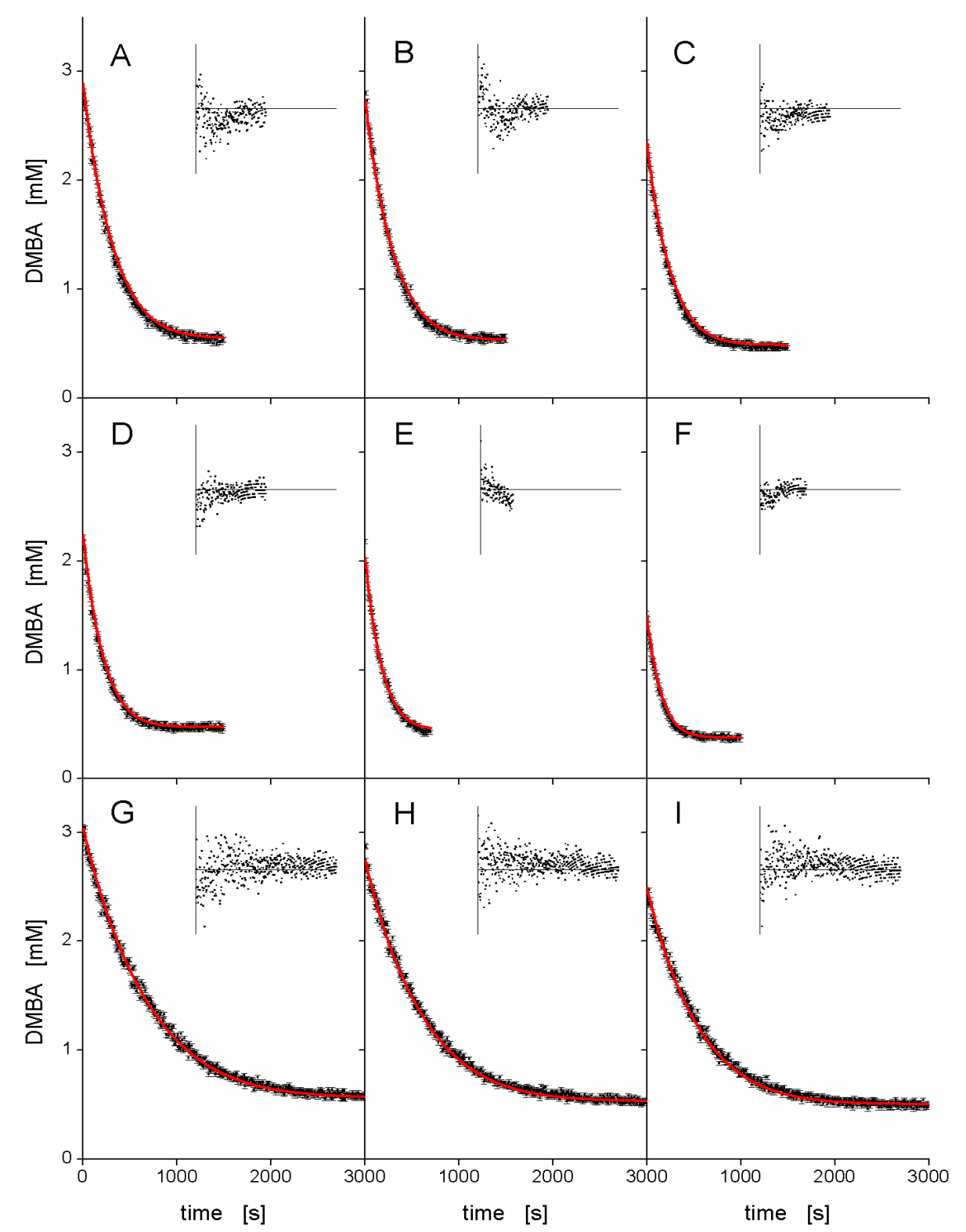

Figure 2-12: Comparison of the fitted simplified kinetic model with the experimental data using experiments A-I (Table 2-4). The residuals are shown as small inlets.

Table 2-12: $\quad$ Parameter estimates and standard deviations using the simplified kinetic model.

\begin{tabular}{lll}
\hline Parameter & Unit & \multicolumn{1}{l}{ Estimate } \\
\hline$k_{\text {catf }}$ & $\mathrm{s}^{-1}$ & $45.443 \pm 0.175$ \\
$K_{e q}$ & $\mathrm{mM}^{-1}$ & $3.965 \pm 0.014$ \\
$K_{m A}$ & $\mathrm{mM}$ & $0.012 \pm 0.003$ \\
$K_{m B}$ & $\mathrm{mM}$ & $0.047 \pm 0.007$ \\
\hline
\end{tabular}

Obviously, those parameters can now be estimated much more precisely despite the tight experimental limitations. In Table 2-13 the correlation matrix is presented. The obtained 
correlations between the kinetic parameters are satisfactory except for the correlation of $K_{m A}$ and $K_{m B}$.

Table 2-13: Correlation matrix of the estimated parameters using the simplified kinetic model.

\begin{tabular}{lllll}
\hline & $k_{\text {catf }}$ & $K_{\text {eq }}$ & $K_{m A}$ & $K_{m B}$ \\
\hline$k_{\text {catf }}$ & 1.00 & 0.54 & 0.69 & 0.68 \\
$K_{\text {eq }}$ & & 1.00 & 0.40 & 0.39 \\
$K_{m A}$ & & & 1.00 & 1.00 \\
$K_{m B}$ & & & & 1.00 \\
\hline
\end{tabular}

With the Eqs. 2-20-23 the micro-reaction rate constants were calculated:

$$
\begin{gathered}
k_{1}=k_{2}=\frac{k_{\text {catf }}}{K_{m A}} \\
k_{-1}=k_{-2}=\frac{k_{\text {catf }} \cdot\left(K_{m B}-K_{m A}\right)}{K_{m A}} \\
k_{3}=k_{\text {catf }} \\
k_{-3}=\frac{k_{\text {catf }}}{K_{e q} \cdot\left(K_{m B}-K_{m A}\right)^{2}}
\end{gathered}
$$

For the error propagation the Gauss formula cannot be used since there are correlations between the parameters. Therefore, Eq. 2-24 was used instead (Tellinghuisen, 2001).

$$
\sigma_{k}^{2}=J^{T} C J
$$

In Eq. 2-24, $\sigma_{k}^{2}$ represents the variance in the function for $k_{i}$ (Eqs. 2-20-23) containing the model parameters $\theta_{i}$, whose variance-covariance matrix is $C_{i, j}$ (Table 2-14). The Jacobian matrix $J$ contains the partial derivatives of $k_{i}$ with respect to the model parameters $\theta_{i}$ :

$$
J_{i}=\frac{\partial k}{\partial \theta_{i}}
$$


Table 2-14: Variance-covariance matrix of the estimated parameters using the simplified kinetic model.

\begin{tabular}{lllll}
\hline & $k_{\text {catf }}$ & $K_{e q}$ & $K_{m A}$ & $K_{m B}$ \\
\hline$k_{\text {catf }}$ & $3.18 \cdot 10^{-2}$ & $1.35 \cdot 10^{-3}$ & $3.53 \cdot 10^{-4}$ & $8.69 \cdot 10^{-4}$ \\
$K_{e q}$ & & $2.01 \cdot 10^{-4}$ & $1.64 \cdot 10^{-5}$ & $3.94 \cdot 10^{-5}$ \\
$K_{m A}$ & & & $8.20 \cdot 10^{-6}$ & $2.06 \cdot 10^{-5}$ \\
$K_{m B}$ & & & & $5.18 \cdot 10^{-5}$ \\
\hline
\end{tabular}

Consequently, the standard deviation of a micro-reaction rate constant $\sigma_{k}$ can be calculated with the following equation:

$$
\sigma_{k}=\left(\sum_{i=1}^{n} \sum_{j=1}^{n}\left(\frac{\partial k}{\partial \theta_{i}} \frac{\partial k}{\partial \theta_{j}} C_{i, j}\right)\right)^{\frac{1}{2}}
$$

Table 2-15: $\quad$ Calculated micro-reaction rate constants.

\begin{tabular}{lccl}
\hline Micro-reaction rate constant & Calculated value & Standard deviation & Unit \\
\hline$k_{1}, k_{2}$ & 3786.9 & 893.7 & $\mathrm{mM}^{-1} \mathrm{~s}^{-1}$ \\
$k_{-1}, k_{-2}$ & 132.5 & 14.9 & $\mathrm{~s}^{-1}$ \\
$k_{3}$ & 45.4 & 0.2 & $\mathrm{~s}^{-1}$ \\
$k_{-3}$ & 9355.9 & 2306.6 & $\mathrm{mM}^{-1} \mathrm{~s}^{-1}$ \\
\hline
\end{tabular}

The calculated values for the micro-reaction rate constants (Table 2-15) are visualized using Cleland's notation (1963) in Figure 2-13. It indicates that the release of the product is ratelimiting for the synthesis reaction, while the release of the substrates is rate-limiting for the reverse reaction.

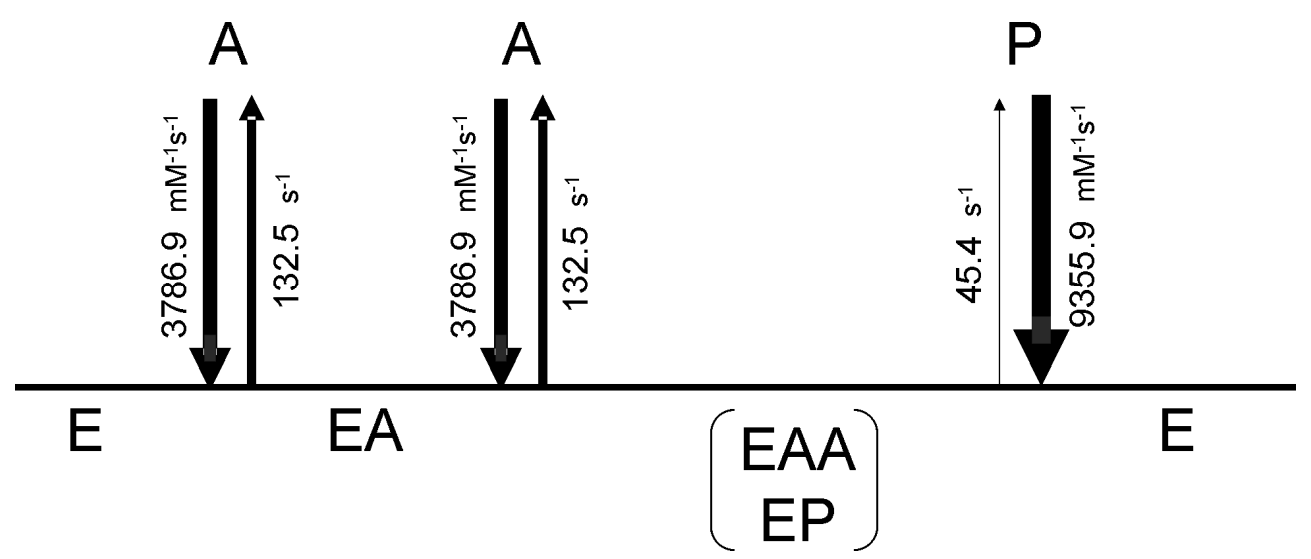

Figure 2-13: $\quad$ Visualization of micro-reaction steps in the BAL mechanism using Cleland's notation (1963). 
Despite the fact that the parameter estimation was much more precise, it can be expected that the estimation would be even more precise if the experimental degrees of freedom would not be so limited. Therefore, new in silico experiments (Table 2-16) were designed for fitting the simplified model. For the model-based optimal experimental design the same experimental limitations have been used. Once more, the determined optimal experiments have been conducted in silico.

Table 2-16: Optimal design for in silico experiments using the simplified kinetic model (R-Y). For the optimal experimental design the degrees of freedom were the initial substrate concentration $c_{A 0}$ (limits: 0-5 $\mathrm{mM}$ ), the initial product concentration $c_{P 0}$ (limits: $0-5 \mathrm{mM}$ ) and the measurement duration $t$ (limits: $50-640 \mathrm{~s}$ ). The enzyme concentration was held constant at $1.25 \cdot 10^{-4} \mathrm{mM}$.

\begin{tabular}{llll}
\hline Experiment & $c_{A O}[\mathrm{mM}]$ & $c_{P O}[\mathrm{mM}]$ & $t[\mathrm{~s}]$ \\
\hline $\mathrm{R}$ & 0.38 & 0.00 & 380 \\
$\mathrm{~S}$ & 0.00 & 4.80 & 620 \\
$\mathrm{~T}$ & 0.41 & 0.00 & 410 \\
$\mathrm{U}$ & 2.84 & 0.31 & 380 \\
$\mathrm{~V}$ & 3.00 & 0.00 & 320 \\
$\mathrm{~W}$ & 0.00 & 4.30 & 292 \\
$\mathrm{X}$ & 2.37 & 0.16 & 310 \\
$\mathrm{Y}$ & 0.00 & 0.21 & 50 \\
\hline
\end{tabular}

The simulated data have been disturbed with white noise with a standard deviation of $0.001 \mathrm{mM}$ and used for a new parameter estimation. The results of this step are presented in Tables $2-17$ and 2-18.

Table 2-17: Parameter estimates and standard deviations after performing optimal in silico experiments using the simplified kinetic model.

\begin{tabular}{lll}
\hline Parameter & Unit & Estimate \\
\hline$k_{\text {catf }}$ & $\mathrm{s}^{-1}$ & $45.438 \pm 0.019$ \\
$K_{\text {eq }}$ & $\mathrm{mM}^{-1}$ & $3.989 \pm 0.003$ \\
$K_{m A}$ & $\mathrm{mM}$ & $0.010 \pm 0.0001$ \\
$K_{m B}$ & $\mathrm{mM}$ & $0.042 \pm 0.0003$ \\
\hline
\end{tabular}


Table 2-18: Correlation matrix of the estimated parameters after performing optimal in silico experiments using the simplified kinetic model.

\begin{tabular}{lllll}
\hline & $k_{\text {catf }}$ & $K_{\text {eq }}$ & \multicolumn{1}{c}{$K_{m A}$} & $K_{m B}$ \\
\hline$k_{\text {catf }}$ & 1.00 & -0.62 & 0.08 & 0.04 \\
$K_{e q}$ & & 1.00 & -0.53 & -0.51 \\
$K_{m A}$ & & & 1.00 & 1.00 \\
$K_{m B}$ & & & & 1.00 \\
\hline
\end{tabular}

Table 2-17 implies that the parameter precision can be increased if the model is fitted to experiments with initial product concentrations. The correlations become slightly lower, but the correlation between $K_{m A}$ and $K_{m B}$ still remains high (Table 2-18). Considering the definition of these parameters, the remaining high correlation is not surprising since $K_{m A}$ and $K_{m B}$ both contain the term $k_{3} / k_{1}$ (Table 2-11).

For the simplified model, a new dynamic sensitivity analysis was performed, which is depicted in Figure 2-14. As already observed for the basic model (Figure 2-11), the parameters $k_{\text {catf }}$ and $K_{e q}$ can be estimated very precisely since they are very sensitive at the start and at the end of the reaction, respectively. The strong correlation of $K_{m A}$ and $K_{m B}$ is caused by the symmetrical sensitivity curves of these parameters. A possible solution for this could be the modeling of the enzyme reaction directly with micro-reaction rate constants, which will be studied in the following Section 2.3.

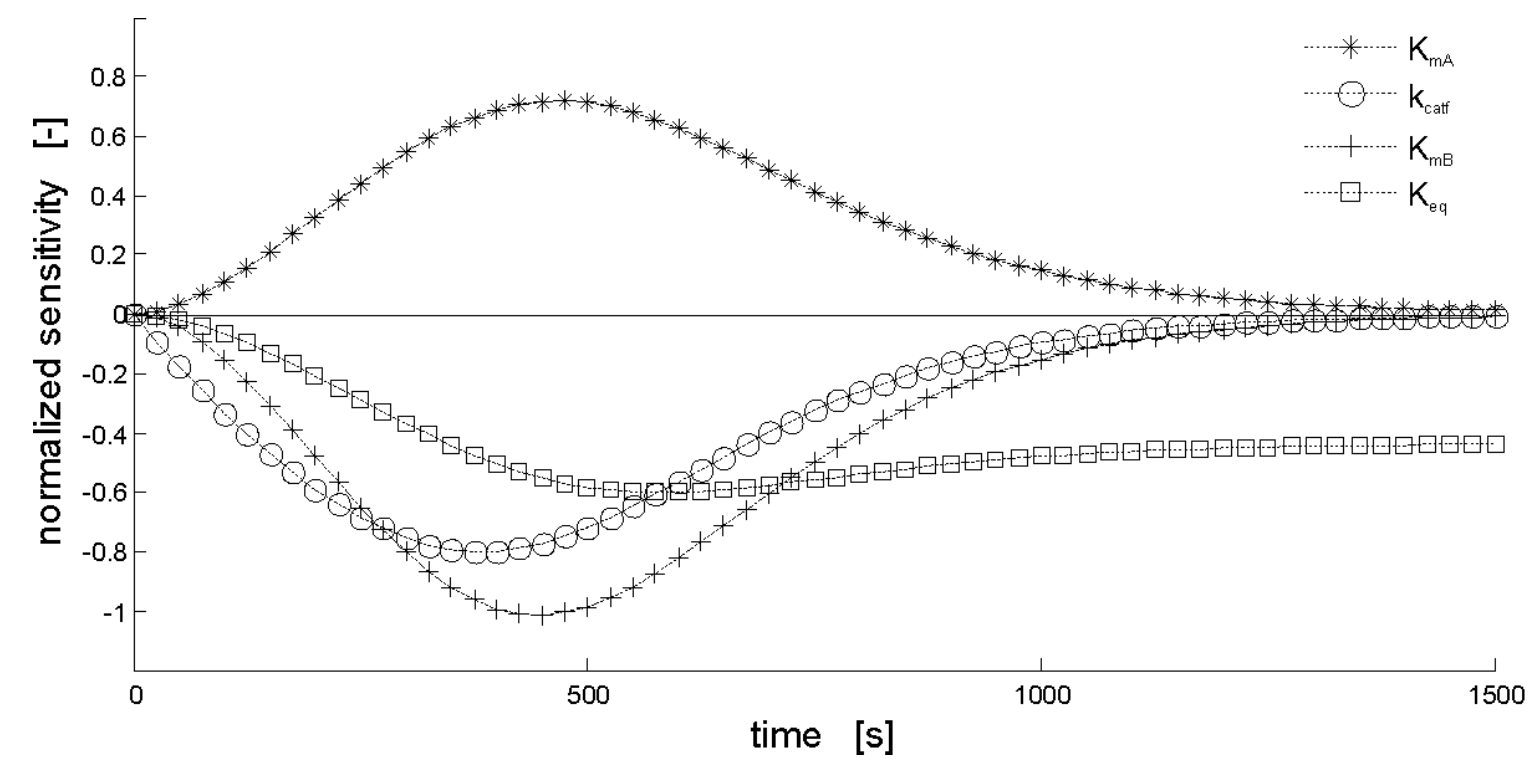

Figure 2-14: $\quad$ Normalized sensitivities of the model parameters of the simplified kinetic model regarding the substrate concentration. 


\subsubsection{Conclusions}

Based on the proposed mechanism for BAL, a kinetic model was derived, which takes all micro-reaction steps into account. In the special case of two identical substrates, two $K_{m^{-}}$ values exist since one substrate acts as donor and the other as acceptor. The model contains six independent parameters. Four of them cannot be estimated with sufficient precision. There are two reasons for this. First the experimentally accessible region is rather limited due to the low solubility of the product. This reason can be investigated with the use of optimally designed in silico experiments, but even under these optimal conditions the model is too complex for estimating all six parameters with high precision. For this reason the model was revised by assuming identical values for $k_{1}$ and $k_{2}$, and for $k_{-1}$ and $k_{-2}$. Therefore, only four independent parameters remain, which can be estimated much more precisely. In silico experiments show that under less limited experimental conditions the model parameters can be estimated very precisely. The derived kinetic model could also be applied for other ThDPdependent enzymes. Most of these enzymes basically follow the same mechanism with the only difference that they are not able to cleave the formed $(R)$-2-hydroxy ketones.

\subsection{Kinetic modeling of benzaldehyde lyase with micro- reaction rate constants}

\subsubsection{Introduction}

As shown in the previous Section, a mechanistic kinetic model was derived for benzaldehyde lyase (BAL, EC 4.1.2.38). The mechanistic kinetic model bases on the microscopic reaction steps of the enzymatic mechanism (Figure 2-7) and contains six independent model parameters which are functions of the six micro-reaction rate constants. However, not all six model parameters are identifiable. By introducing the assumption that the binding and the release of the first and the second substrate molecule are equally fast, the four remaining independent model parameters can be estimated with high precision and the micro-reaction rate constants can be calculated. Alternatively the model can directly be developed on the micro-reaction level. 
The direct use of micro-reaction rate constants is uncommon. The most important reason for this is that as long as no advanced tools for parameter estimation were available, the initial rate method was employed. Thus, the model parameters could directly be obtained using graphical data analysis such as Lineweaver-Burk plots (1934). Nowadays, sophisticated analysis tools like those described in Section 2.1 exist and progress curve analysis can be applied. In contrast to the initial rate method, all parameters are estimated simultaneously. In this respect, the estimation of micro-reaction rate constants instead of macroscopic model parameters makes no difference. Nevertheless, most scientists are used to macroscopic parameters such as $K_{m}$-values for almost a century and keep on using them with only few exceptions (Bauer et al., 2002; Dingee and Anton, 2008).

When deriving a kinetic model for enzyme reactions it is usually assumed that the concentrations of the free enzyme and intermediates do not change with time. This so-called steady-state assumption reduces the complexity of the kinetic model (Dingee and Anton, 2008). Thus, relatively simple kinetic models can be derived by applying the method of King and Altman (1956). In contrast, the kinetic models without the steady-state assumption are much more complex since differential equations for the free enzyme and intermediates have to be included. On the other hand, the steady-state assumption causes an error, because the intermediates have to be formed at the beginning of the reaction. Consequently, the pros and cons of the direct use of micro-reaction rate constants are discussed on the example of BAL, and the error is quantified which is caused by the steady-state assumption.

\subsubsection{Micro-kinetic model}

As shown in Figure 2-7 C, BAL follows a bi-uni mechanism. The micro-kinetic model contains the Eqs. 2-27-31 and was fitted to the same experimental data as the derived macrokinetic model (Table 2-4). The influences of the steady-state assumption and of the mechanistic assumption $\left(k_{1}=k_{2}\right.$ and $\left.k_{-1}=k_{-2}\right)$ from Section 2.2.3.3 were investigated. 


$$
\begin{aligned}
& \frac{d c_{A}}{d t}=-k_{1} \cdot c_{E} \cdot c_{A}+k_{-1} \cdot c_{E A}-k_{2} \cdot c_{E A} \cdot c_{A}+k_{-2} \cdot c_{E A A} \\
& \frac{d c_{P}}{d t}=k_{3} \cdot c_{E A A}-k_{-3} \cdot c_{E} \cdot c_{P} \\
& \frac{d c_{E}}{d t}=-k_{1} \cdot c_{E} \cdot c_{A}+k_{-1} \cdot c_{E A}+k_{3} \cdot c_{E A A}-k_{-3} \cdot c_{E} \cdot c_{P} \\
& \frac{d c_{E A}}{d t}=k_{1} \cdot c_{E} \cdot c_{A}-k_{-1} \cdot c_{E A}+k_{-2} \cdot c_{E A A}-k_{2} \cdot c_{E A} \cdot c_{A} \\
& c_{E 0}=c_{E}+c_{E A}+c_{E A A}
\end{aligned}
$$

A sensitivity analysis was performed as described in Section 2.2.3.2. To determine the error caused by the steady-state assumption, simulations were performed either with or without the steady-state assumption using gPROMS (version 3.0.2, Process System Enterprise Ltd., London, UK). In the latter case, it was assumed that at the start of the reaction only free enzyme is present.

\subsubsection{Results and discussion}

\subsubsection{Parameter estimation}

The results of the parameter estimations to the experimental data (experiments A-I, Table 2-4) are listed in Table 2-19. Obviously, the steady-state assumption has no significant influence on the estimated values. Their standard deviations are only marginally smaller. Moreover, the introduction of the mechanistic assumption does not affect the obtained estimates considerably. However, as already observed for the macro-kinetic model in Section 2.2, some parameters are not identifiable without the mechanistic assumption meaning that their standard deviations are higher than the estimated values. The results obtained using both assumptions correspond well to the values for micro-reaction rate constants which were calculated from the macroscopic model parameters (Table 2-15). The same is true for the obtained standard deviations, which were calculated in Table 2-15 considering error propagation of correlated parameters (Eq. 2-26). Each parameter estimation indicates that the product release is rate-limiting which supports the result of Section 2.2. 
Table 2-19: $\quad$ Estimated values of the micro-reaction rate constants and their standard deviations. The estimations were performed either with or without the steady-state assumption and either with or without the mechanistic assumption introduced in Section 2.2.3.3.

\begin{tabular}{|c|c|c|c|c|c|}
\hline & \multicolumn{2}{|c|}{ without steady-state assumption } & \multicolumn{2}{|c|}{ with steady-state assumption } & \multirow[t]{2}{*}{ unit } \\
\hline & $\begin{array}{l}k_{1} \neq k_{2} \\
k_{-1} \neq k_{-2}\end{array}$ & $\begin{array}{l}k_{1}=k_{2} \\
k_{-1}=k_{-2}\end{array}$ & $\begin{array}{l}k_{1} \neq k_{2} \\
k_{-1} \neq k_{-2}\end{array}$ & $\begin{array}{l}k_{1}=k_{2} \\
k_{-1}=k_{-2}\end{array}$ & \\
\hline$k_{1}$ & $3764.6 \pm 136963.1$ & & $3762.0 \pm 135918.7$ & & $\mathrm{mM}^{-1} \mathrm{~s}^{-1}$ \\
\hline$k_{2}$ & $3937.6 \pm 9889.4$ & $3799.9 \pm 886.6$ & $3935.3 \pm 9782.0$ & $3800.0 \pm 886.3$ & $\mathrm{mM}^{-1} \mathrm{~s}^{-1}$ \\
\hline$k_{-1}$ & $142.9 \pm 2185.1$ & & $142.8 \pm 2169.6$ & & $\mathrm{~s}^{-1}$ \\
\hline$k_{-2}$ & $126.4 \pm 1628.3$ & $133.2 \pm 15.0$ & $126.3 \pm 1619.5$ & $133.2 \pm 15.0$ & $\mathrm{~s}^{-1}$ \\
\hline$k_{3}$ & $45.7 \pm 1.0$ & $45.5 \pm 0.2$ & $45.8 \pm 1.0$ & $45.5 \pm 0.2$ & $\mathrm{~s}^{-1}$ \\
\hline$k_{-3}$ & $9421.7 \pm 342761.9$ & $9346.1 \pm 2252.9$ & $9425.0 \pm 340508.6$ & $9345.5 \pm 2252.0$ & $\mathrm{mM}^{-1} \mathrm{~s}^{-1}$ \\
\hline
\end{tabular}

Table 2-20: Correlation matrix of estimated micro-reaction rate constants using the steady-state and the mechanistic assumption.

\begin{tabular}{lllll}
\hline & $k_{1}$ & $k_{-1}$ & $k_{3}$ & $k_{-3}$ \\
\hline$k_{1}$ & 1.00 & 1.00 & -0.68 & 1.00 \\
$k_{-1}$ & & 1.00 & -0.70 & 1.00 \\
$k_{3}$ & & & 1.00 & -0.66 \\
$k_{-3}$ & & & & 1.00 \\
\hline
\end{tabular}

The correlation matrix of the micro-kinetic model using both assumptions is shown in Table 2-20. The high correlations of the parameters are typical for consecutive reactions and make the estimation of accurate values for the parameters difficult (Franceschini and Macchietto, 2008d). All parameter pairs exhibit correlations with absolute values of at least 0.66. This is in contrast to the correlation matrix of the macroscopic parameters (Table 2-13), where only three pairs of different parameters exhibit correlations higher than 0.66 . Moreover, using the micro-kinetic model correlations of 1.00 are observed for the pairs $k_{1}: k_{-1}, k_{1}: k_{-3}$, and $k_{-1}: k_{-3}$, while a correlation of 1.00 is obtained only for $K_{m A}: K_{m B}$, if the macro-model is employed. 
Therefore, the micro-kinetic model has no advantage in terms of parameter correlations compared to the macro-kinetic model.

\subsubsection{Sensitivity analysis}

A sensitivity analysis for the micro-reaction rate constants was applied using the microkinetic model with both the steady-state assumption and the mechanistic assumption from Section 2.2.3.3. The obtained trajectories are presented in Figure 2-15. If these are compared with those obtained with the simplified macro-model (Figure 2-14), it can be seen that the micro-reaction rate constants show different sensitivity trajectories compared to the macroscopic model parameters. The most important difference is that all four micro-reaction rate constants remain sensitive at equilibrium. In contrast to this, the macroscopic model parameter $K_{e q}$ is the only parameter which determines the equilibrium position. Thus, $K_{e q}$ can be estimated separately, while the micro-reaction rate constants cannot be estimated individually.

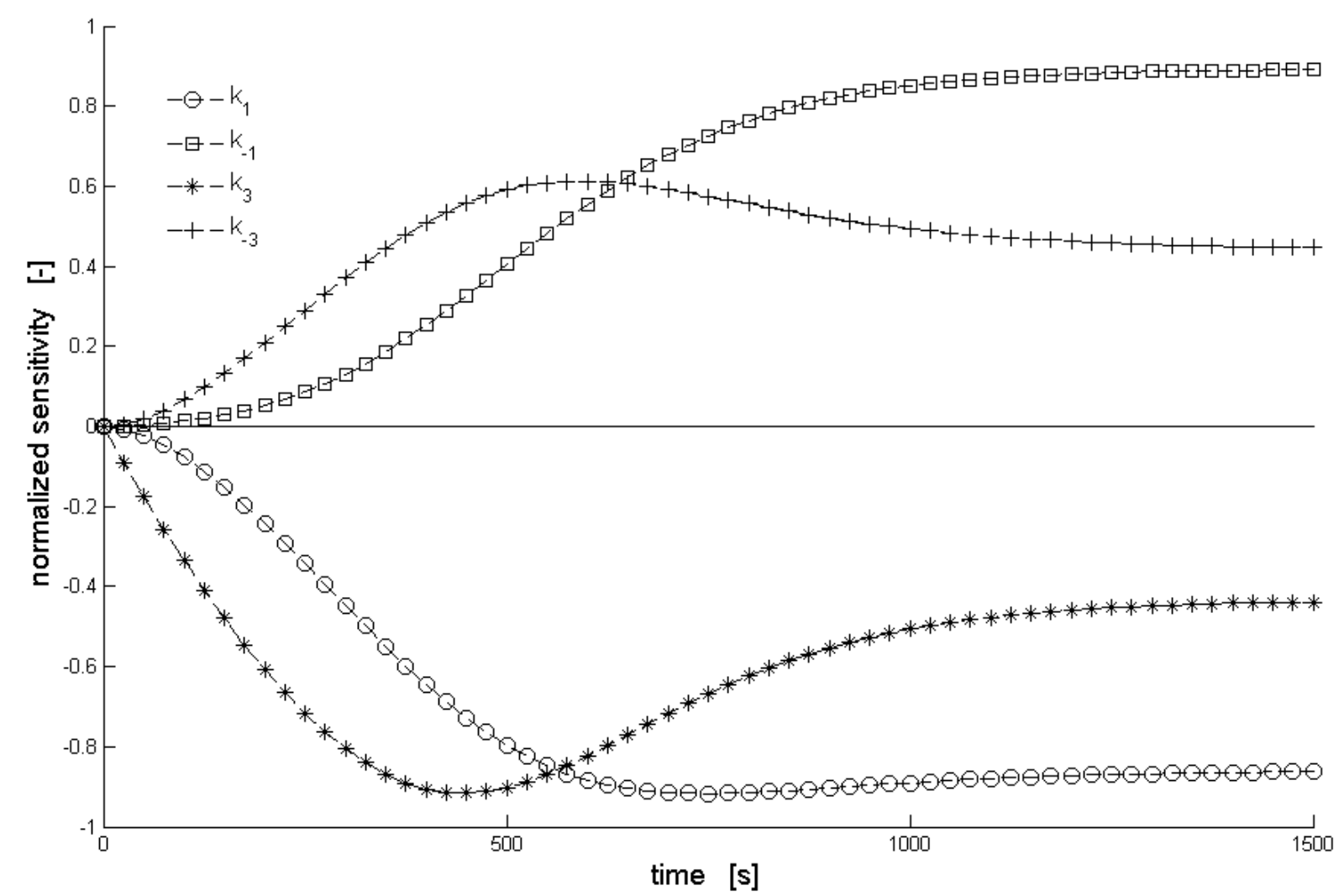

Figure 2-15: Normalized sensitivities of the micro-reaction rate constants using the micro-kinetic model with both the steady-state and the mechanistic assumption. The initial conditions of experiment A (Table 2-4) were used. 


\subsubsection{Error caused by steady-state assumption}

The differences between modeling with and without steady-state assumption are demonstrated in Figure 2-16. Within the first millisecond the substrate concentration decreases faster than predicted by the steady-state model. Moreover, the product concentration increases later, because the enzyme intermediates have to be formed first. Measurements in this transient phase may be very useful to determine the micro-reaction rate constants, however, this requires very advanced methods like the femtosecond pump-probe method (Zhong, 2007). On the other hand, also the quantification of enzyme intermediates in the steady-state region can provide useful information. As demonstrated on the right side of Figure 2-16, the kinetic model predicts the highest concentration of all enzyme intermediates for the ternary complex $(E A A)$ in the steady-state due to the rate-limiting product release.
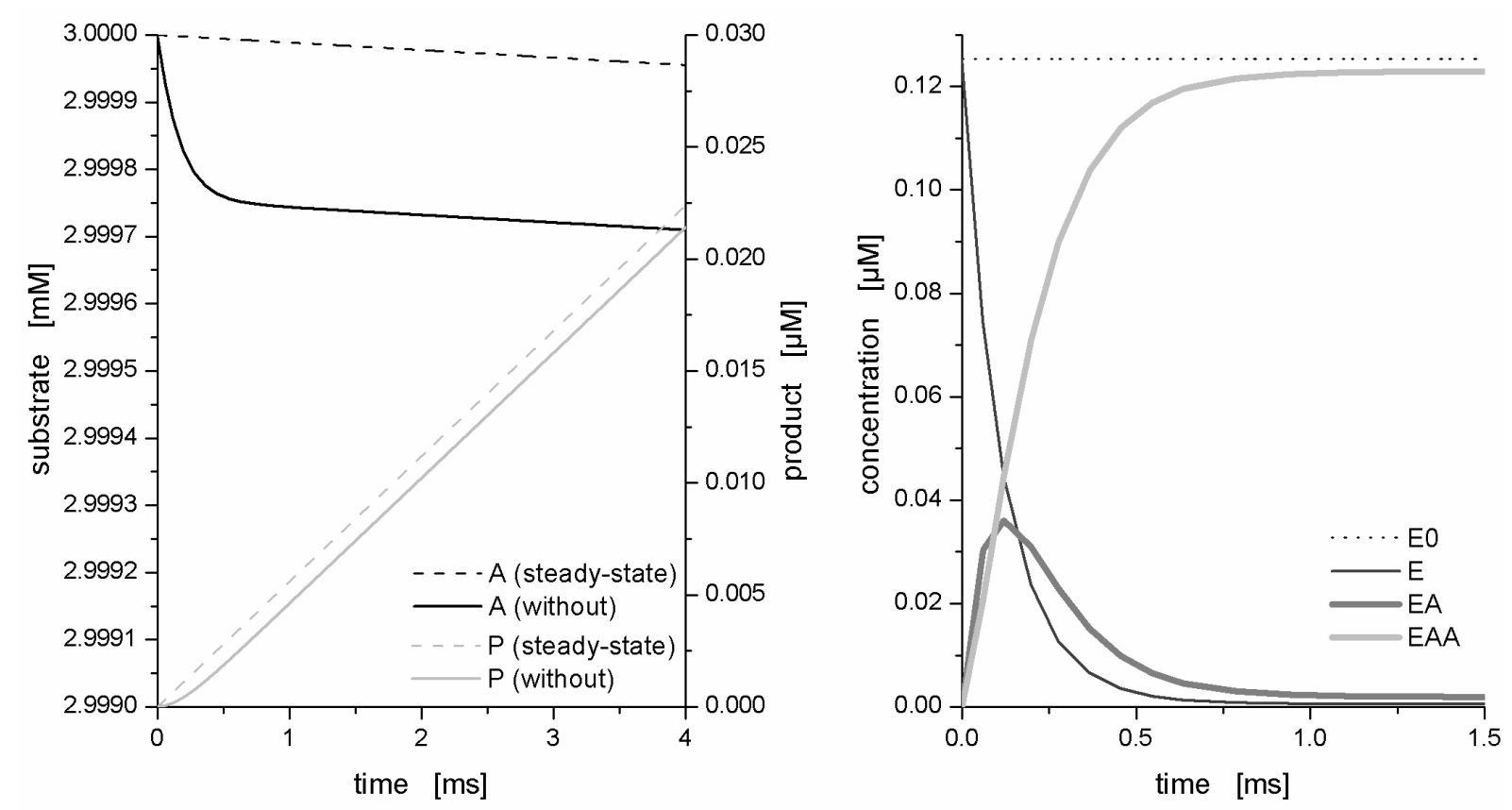

Figure 2-16: Simulated trajectories of substrate and product concentration trajectories with steady-state assumption and without (left). Concentration trajectories of the enzyme intermediates without steady-state assumption (right). The total enzyme concentration is $0.125 \mu \mathrm{M}$, the initial concentrations of substrate and product $3 \mathrm{mM}$ and $0 \mathrm{mM}$, respectively.

After $1 \mathrm{~ms}$ steady-state is achieved since the concentrations of the enzyme intermediates do not change any more. Thereafter the concentration trajectories of both models (with and without steady-state assumption) are parallel to each other. Especially if progress curve analysis is applied, the proportion of the transient phase on the whole measuring time is very 
small. Obviously, the difference of predicted concentrations is very small. The error stays in the micro-molar or nano-molar scale and must always be below twice the total enzyme concentration $\left(\mathrm{c}_{E 0}\right)$ due to the reaction stoichiometry. Since the enzyme concentration is much lower than the concentrations of the reactants and below the detection limit of the used measuring devices, the steady-state error can be neglected in the cases investigated in this project.

The same investigations were performed with two other enzyme mechanisms - a uni-uni mechanism and a bi-bi mechanism. The experimental data for these studies were obtained from industrially important enzymes - phosphoglucose isomerase (Jeong et al., 2003) and formate dehydrogenase (Michalik et al., 2007). Basically the same results were obtained. The maximal error increases with higher substrate and enzyme concentrations, but remains for all reasonable concentrations below the detection limit of standard measuring devices (data not shown).

\subsubsection{Conclusions}

Micro-reaction rate constants provide important information about the enzyme mechanism. Using the mechanistic kinetic model for the symmetric carboligation they can be calculated from macroscopic parameters. Their standard deviations have to be calculated via error propagation for correlated parameters. The alternative is the direct use of micro-reaction rate constants for modeling the enzyme reaction. The advantage of this approach is that they can be obtained directly and that their standard deviations are provided by the computer program. Another advantage might be that model variants can be derived much easier using microreaction rate constants. For example, if one micro-reaction step is assumed to be irreversible, the corresponding micro-reaction rate constant simply has to be set to zero. However, the estimation precision cannot be increased. A drawback of the direct use of micro-reaction rate constants is that all of them are sensitive in equilibrium. Therefore, no parameter can be estimated individually as it is possible for the equilibrium constant $K_{e q}$, if a macro-kinetic model is used.

Macro-kinetic models are based on the steady-state assumption for the free enzyme and enzyme intermediates. It could be demonstrated that steady-state is reached within milliseconds. The maximum concentration error increases with substrate and product 
concentration, but cannot exceed the order of magnitude of the total enzyme concentration. Thus, this steady-state error remains below the detection limit of standard measuring devices and can be neglected in further studies of this thesis. However, it could be shown that the quantification of the enzyme intermediates in the steady-state region can indicate the ratelimiting micro-reaction step.

\subsection{Optimal experimental design for enzyme kinetics of benzaldehyde lyase}

\subsubsection{Introduction}

The accuracy of the kinetic parameters is crucial in enzymology. Only if the kinetic parameters are known accurately, the model reliably predicts the behavior of enzymes. This is not only essential for design, optimization, and control of industrial processes using enzymes, but also in the medical and pharmaceutical research for the prediction of toxic or metabolic effects in the human body. Since incorrectly designed experiments can cause poor parameter estimates, the need for well-designed enzymatic experiments is becoming increasingly recognized in research (Murphy et al., 2003). An optimal design saves resources such as time, money, and materials, and generates parameter estimates with high precision. Moreover, possible model variants can be discriminated (Franceschini and Macchietto, 2007, 2008a, 2008b; Walter and Pronzato, 1990). To date, most studies on experimental design of enzyme kinetics focus on initial rate measurements and are based on qualitative considerations rather than rigorous numerical methods (Alberty, 2008; Murphy et al., 2003). In contrast to this, only few studies apply optimal experimental design (OED) techniques for optimizing progress curve measurements (Duggleby and Clarke, 1991; Lindner and Hitzmann, 2006). Duggleby and Clarke (1991) determined a practical guideline for designing progress curve measurements, if the enzyme mechanism follows an irreversible uni-uni mechanism. Accordingly, the initial substrate concentration should be 2-3 times higher than the $K_{m}$-value and data should be collected until at least $90 \%$ conversion is achieved. However, so far no general rules exist for more complex mechanisms such as symmetric carboligation reactions.

In Section 2.2.3.2 the OED of new experiments for benzaldehyde lyase (BAL) was used to demonstrate that the six model parameters of the derived mechanistic kinetic model are not 
identifiable. After model revision, OED was applied to show that the precision of the obtained parameters can be improved in an experimentally less restricted setting (Section 2.2.3.3). The OED for such symmetric carboligation reactions is further investigated in this Section, which facilitates the experimental design if new enzymes or reactants are utilized that are not experimentally limited by their solubility.

\subsubsection{Analysis procedure}

The general influences of several factors such as the number of experiments, the number of data points per experiment, and the standard deviation of the experimental data points were investigated using the simplified kinetic model. For the OED the E-criterion, which minimizes the largest eigenvalue of the variance-covariance matrix, was chosen. Thus, the optimized experiments focus on improving the precision of those parameters that are most uncertain (Asprey and Macchietto, 2000; Walter and Pronzato, 1990). The design space ranged from $0-20 \mathrm{mM}$ for the initial substrate and product concentration, and between 0 $6000 \mathrm{~s}$ for the measurement duration. All experiments were conducted in silico. In order to evaluate the OEDs, the Student-t-values were calculated (Eq. 2-32):

$$
\text { Student }-t-\text { value }=\frac{\theta_{i}}{95 \% \text { confidence interval }}
$$

The basic setting used for the OED consisted of three experiments, 500 data points per experiment, and a standard deviation of $0.05 \mathrm{mM}$ for each measurement point. To investigate the influences of these three factors, one was varied while the others were kept constant. The CPU time to perform an OED was determined using a standard PC (800 MHz).

\subsubsection{Results and discussion}

Figure 2-17 visualizes the design space and the optimized experiments for investigated symmetric carboligation using BAL. The thermodynamic equilibrium (Eq. 2-15) can be recognized by a minimum of the reaction rate (dashed line). Thus, all designed experiments cannot be conducted beyond this curve. The designed experiments (Table 2-16) can be divided into two groups. The first group of experiments (R, T, and Y) are conducted with very low reactant concentrations and thus focus on the improvement of the $K_{m}$-values, which are 
most sensitive at low concentrations. The other group of experiments $(\mathrm{S}, \mathrm{U}, \mathrm{V}, \mathrm{W}$, and $\mathrm{X})$ start with much higher initial concentrations. Hence, the maximum turnover number $k_{\text {catf }}$ can be determined and in the course of the reaction a wide range of reaction rates is passed. Moreover, it can be seen that the designed experiments are arranged in a way which allows determining the curvature of the equilibrium line precisely.

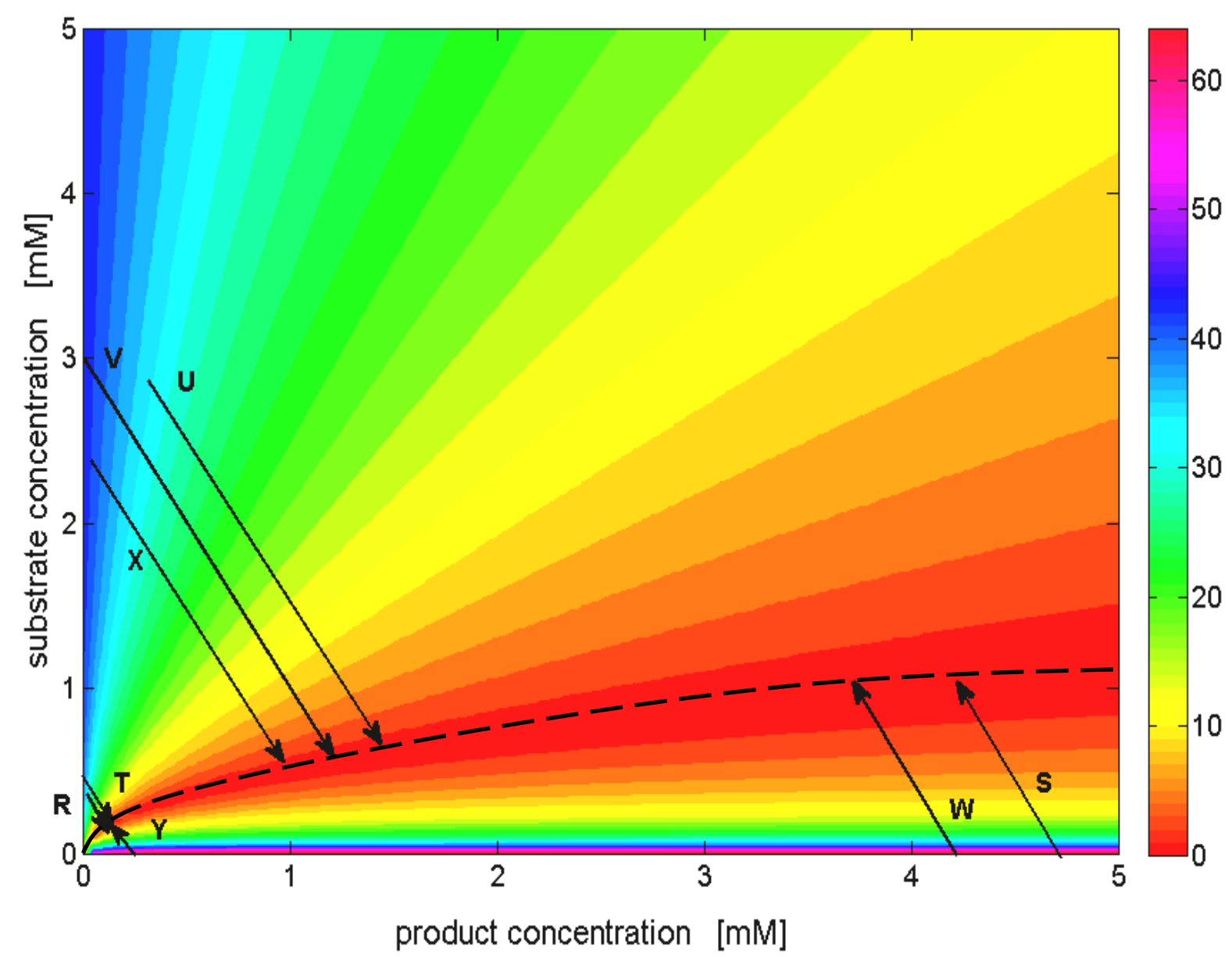

Figure 2-17: Plot of the reaction rate over the substrate and product concentration as contour plot. The reaction rate was calculated using the simplified kinetic model (Section 2.2.3.3) and the parameter values listed in Table 2-12. The color bar indicates reaction rates in $\mathrm{mM} \mathrm{s}^{-1}$. A reaction rate of $0 \mathrm{mM} \mathrm{s}^{-1}$ indicates thermodynamic equilibrium (dashed line). The optimal experiments are visualized by arrows. The experiments R-Y (Table 2-16) were designed as described in Section 2.2.3.2.

Based on these results, it can be concluded that it is beneficial to design experiments both with substrate and product being initially present. Some experiments should be performed in the range of the $K_{m}$-values, while others should be conducted with high initial substrate and product concentrations. Moreover, the stoichiometry of the reaction should be considered. For 
instance, an experiment with an initial substrate concentration of $4 \mathrm{mM}$ results in the same thermodynamic equilibrium as an experiment with an initial product concentration of $2 \mathrm{mM}$. Experiments should be designed so that the equilibrium position is reached at different combinations of reactants in order to determine the curvature of the equilibrium line precisely.

Besides the arrangement of the optimized experiments, the experimenter is confronted with the question, how many experiments should be conducted, what precision of the measuring device is needed and how many data points should be recorded (Franceschini and Macchietto, 2008d). Figure 2-18 shows general trends to answer these questions.
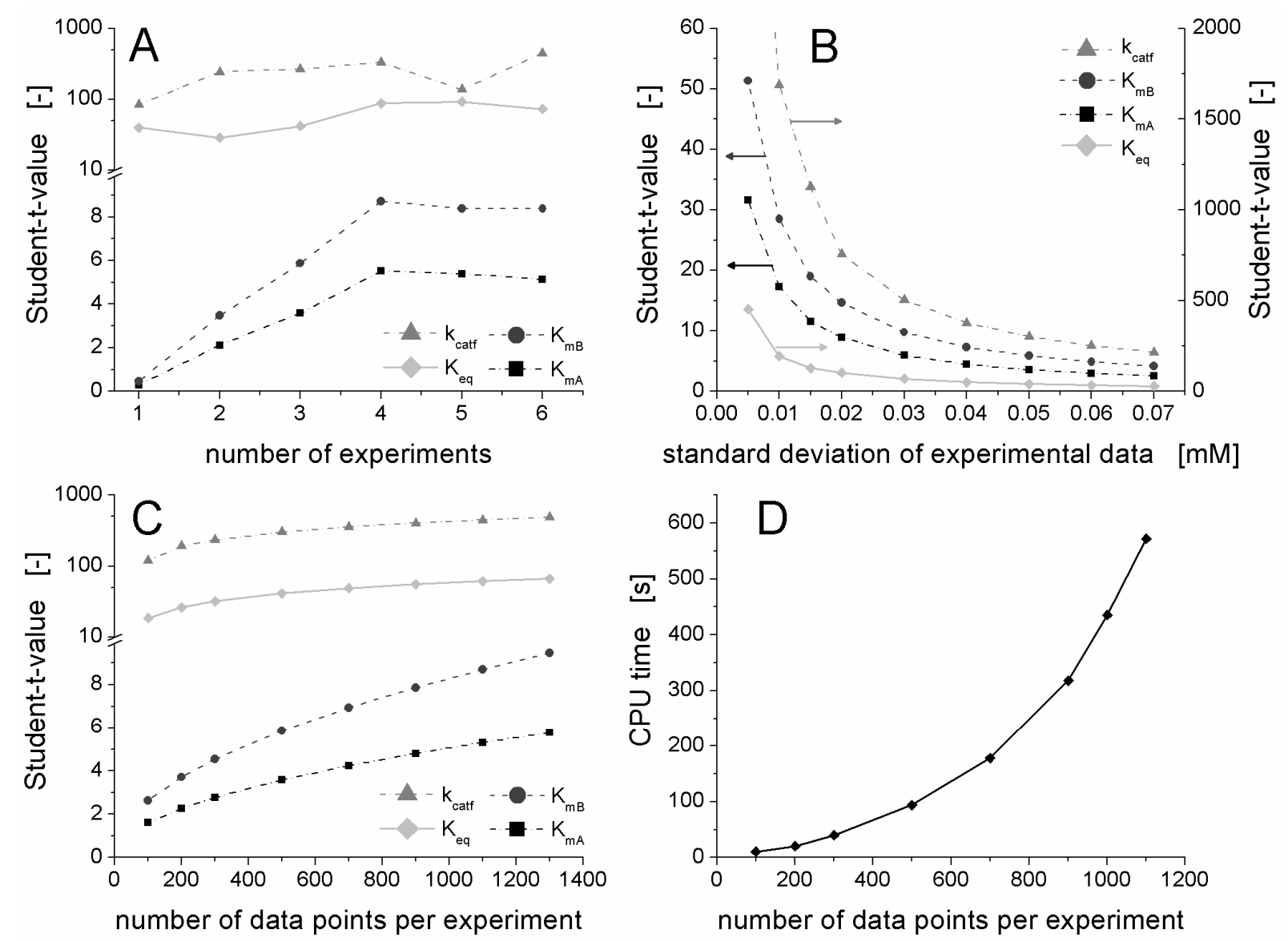

Figure 2-18: Influences of the number of experiments (A), the standard deviation of the experimental data (B), and the number of data points per experiment (C) on the precision of the obtained parameter estimates using the simplified kinetic model for BAL. The CPU time needed for OED is plotted versus the number of data points (D).

If more optimized experiments are designed, the precision of the model parameters increases (Figure 2-18A). It can be seen that the model parameters with the highest uncertainty like $K_{m A}$ 
and $K_{m B}$ are improved most significantly, while the precision of the already precise estimate for $K_{e q}$ is almost not enhanced. This is in accordance with the algorithm of the used Ecriterion, which minimizes the largest eigenvalue of the variance-covariance matrix, which results in a reduction of the uncertainties of the model parameters with the lowest precision (Franceschini and Macchietto, 2008a). Four experiments can be sufficient to achieve satisfying precisions of the kinetic parameters, more experiments do not enhance the precision notably. The standard deviation of the experimental data contributes to the calculation of the variance-covariance matrix (Box and Lucas, 1959). Therefore, the enhancement of the precision of a measuring device improves the reliability of the obtained parameter estimates drastically (Figure 2-18B). Moreover, the plot of the Student-t-values over measurement points shows that in general as much data points as possible should be collected (Figure 2-18C). However, the computational effort for OED increases nearly quadratically (Figure 2-18D).

As already done in Section 2.3, these investigations were also performed using a uni-uni mechanism and a bi-bi mechanism. The parameter values for these studies were obtained from phosphoglucose isomerase (Jeong et al., 2003) and from formate dehydrogenase (Michalik et al., 2007). These studies confirm the observed trends (data not shown).

\subsubsection{Conclusions}

The investigation of the OED for symmetric carboligations on the example of BAL reveals several guidelines. First of all, experiments should be designed with both initial substrate and product concentrations. Some experiments should be conducted in the concentration range of the $K_{m}$-values, while others should start with high initial concentrations. The initial concentrations should be chosen in a way that different combinations of reactant concentrations are obtained in equilibrium. In general the precision of estimated parameters can be improved with increasing number of experiments and measurement points and with applying precise measuring devices. To estimate the kinetic parameters of the simplified kinetic model for BAL, four experiments can be sufficient. Since Lindner and Hitzmann (2006) demonstrate that substrate feeding may improve the precision of parameter estimation for enzyme kinetics significantly, further investigations should focus on sophisticated feeding profiles or pulses of reactants. 


\subsection{Dimethylformamide as cosolvent}

\subsubsection{Introduction}

As demonstrated in the previous Section, the precision of the parameter estimates can be increased by performing experiments with initial substrate and product concentrations. However, the enantiopure product is commercially not available. For this reason, enantiopure product was synthesized using BAL and then purified (Schmidt, 2008). Moreover, higher solubilities enlarge the design space for the optimal experimental design (OED) for homogeneous reaction systems as well as biphasic reaction systems. Therefore, a cosolvent screening was conducted which revealed that the solubilities of both substrate and product are increased if dimethylformamide (DMF) instead of dimethylsulfoxide (DMSO) is used (Schmidt, 2008). However, this makes it necessary to re-estimate the kinetic parameters of the kinetic model derived in Section 2.2.3.1. Additionally, this study reveals, how the cosolvent DMF affects the kinetic parameters of benzaldehyde lyase and if the availability of enantiopure product improves the parameter estimation precision.

\subsubsection{Materials and methods}

The identical chemicals and experimental conditions as described in Section 2.2.2.1 were applied. The only difference was the use of 25\%(v/v) DMF instead of 30\%(v/v) DMSO. The reaction was carried out at $25^{\circ} \mathrm{C}$ in a stirred reactor with a filling volume of $9 \mathrm{~mL}$. The reaction medium was continuously pumped to a fluorimeter (LS55, PerkinElmer, Waltheim, USA), then to a spectrophotometer (SpectraMax Plus, Molecular Devices, Sunnywale, USA), and finally back into the reactor. For the fluorescence measurements an excitation wavelength of $360 \mathrm{~nm}$ was used. Fluorescence emission was quantified at $470 \mathrm{~nm}$. The wavelength for the extinction measurements was $325 \mathrm{~nm}$.

The applied model-based experimental analysis (MEXA) procedure is identical to the one described in Section 2.2. However, the difference was that optimally designed experiments could be performed experimentally instead of in silico. Therefore, the MEXA-cycle could be passed through several times. Accordingly, optimal experiments were designed, conducted, and used for parameter estimation. The fitted model was then used again for another OED. 


\subsubsection{Results and discussion}

\subsubsection{Calibration model}

Systematic differences between kinetic model and experimental data revealed that in presence of DMF the substrate fluorescence is quenched by the product, which in turn exhibits also minor fluorescence (Figure 2-19).

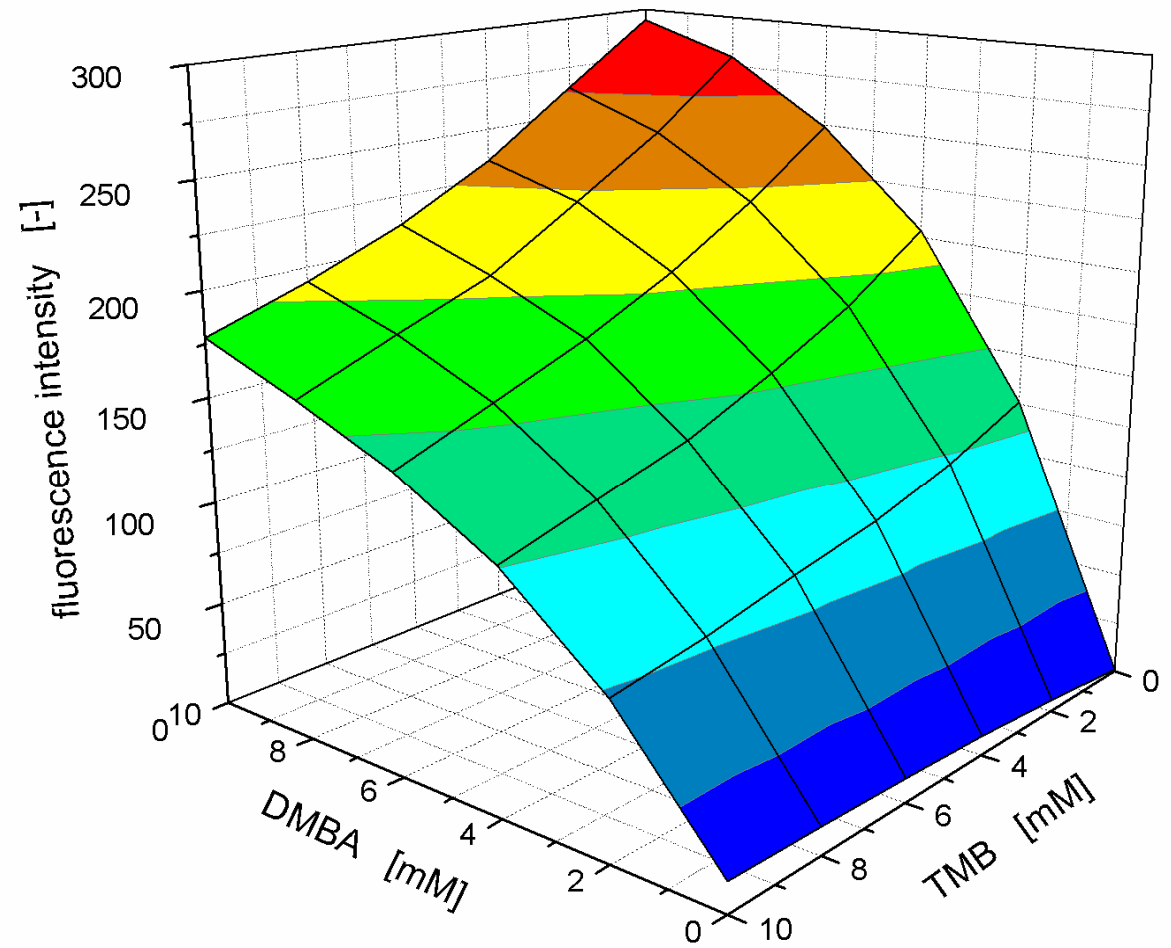

Figure 2-19: Dependence of fluorescence intensity from substrate and product concentration in the presence of DMF.

Therefore, an empirical correlation (Eq. 2-33) was derived and fitted to the calibration data.

$$
I=\left(\left(a \cdot c_{A}^{3}+b \cdot c_{A}^{2}+c \cdot c_{A}\right) \cdot\left(1-d \cdot c_{P}\right)+e \cdot c_{P}^{3}+f \cdot c_{P}^{2}+g \cdot c_{P}\right) \cdot q+s
$$

The parameters $a-g$ were obtained using non-linear regression (Table 2-21). The relative error between calibration model and experimental data is $2.9 \%$. Since the fluorescence intensity fluctuates from day to day, the parameter $q$ takes these fluctuations into account. Using a fluorescence standard this value was estimated separately. The parameter $s$ stands for the background fluorescence, which was determined prior to substrate addition. 
Table 2-21: $\quad$ Parameter estimates of the fluorescence calibration model (excitation wavelength: $360 \mathrm{~nm}$; emission wavelength: $470 \mathrm{~nm}$ )

\begin{tabular}{llc}
\hline Parameter & Unit & Estimate \\
\hline$a$ & $\mathrm{mM}^{-3}$ & 0.20 \\
$b$ & $\mathrm{mM}^{-2}$ & -5.46 \\
$c$ & $\mathrm{mM}^{-1}$ & 64.11 \\
$d$ & $\mathrm{mM}^{-1}$ & 0.05 \\
$e$ & $\mathrm{mM}^{-3}$ & -0.06 \\
$f$ & $\mathrm{mM}^{-2}$ & 1.14 \\
$g$ & $\mathrm{mM}^{-1}$ & -4.00 \\
\hline
\end{tabular}

Since the fluorescence intensity is a function of both reactants, additional information is required in order to be able to calculate the concentrations. Therefore, extinction measurements were conducted using a wavelength of $325 \mathrm{~nm}$. The extinction Ext can be described with the Lambert-Beer law (Eq. 2-34), where $\varepsilon$ denotes the extinction coefficients $\left(2.43 \mathrm{mM}^{-1} \mathrm{~cm}^{-1}\right.$ for DMBA and $2.26 \mathrm{mM}^{-1} \mathrm{~cm}^{-1}$ for TMB) and $d$ the pathlength of the cuvette $(0.5 \mathrm{~mm})$. The background extinction $b$ was determined for each experiment individually.

$$
E x t=\left(\varepsilon_{A} \cdot c_{A}+\varepsilon_{P} \cdot c_{P}\right) \cdot d+b
$$

It is either possible to calculate the concentrations using both equations (Eqs. 2-33 and 2-34) and Newton's method or to include the equations into the kinetic model. Both approaches were tested, however, the latter turned out to be more convenient, because iteratively improved estimations of background parameters do not require repeating Newton's method. Thus, the kinetic model was fitted directly to the fluorescence intensities and extinction values.

The concentrations can also be calculated using the reaction stoichiometry and only one of both equations (Eqs. 2-32 and 2-33). However, this requires the exact knowledge of the initial reactant concentration, which is not always possible due to minor, but inevitable pipetting errors. Moreover, the combination of both measuring techniques improves the reliability of the estimated parameters. 


\subsubsection{Parameter estimation using optimized experiments}

The MEXA cycle was looped four times. In total 29 experiments with different initial conditions were conducted prior to the final cycle. This final MEXA cycle started with the OED of eight experiments using the E-criterion (Table 2-22).

Table 2-22: $\quad$ Optimized experiments: The degrees of freedom for the OED were the initial substrate concentration $c_{A 0}$ (limits: 0-10 $\mathrm{mM}$ ), the initial product concentration $c_{P O}$ (limits: 0-10 mM). the enzyme concentration $c_{E}$ (limits: $10^{-5}-5 \cdot 10^{-4} \mathrm{mM}$ ), and the experiment duration $t_{\exp }$ $(50-10000 \mathrm{~s})$.

\begin{tabular}{lclll}
\hline Experiment & $c_{A 0}[\mathrm{mM}]$ & $c_{P 0}[\mathrm{mM}]$ & $c_{E}[\mathrm{mM}]$ & $t_{\exp }[\mathrm{s}]$ \\
\hline A & 0.10 & 0.32 & $1.17 \cdot 10^{-5}$ & 10000 \\
B & 10.00 & 0.25 & $3.01 \cdot 10^{-5}$ & 10000 \\
C & 9.80 & 0.60 & $1.72 \cdot 10^{-5}$ & 10000 \\
D & 0.50 & 3.00 & $1.71 \cdot 10^{-4}$ & 10000 \\
E & 4.90 & 0.18 & $2.12 \cdot 10^{-5}$ & 8500 \\
F & 2.35 & 0.22 & $1.71 \cdot 10^{-5}$ & 5500 \\
G & 1.15 & 0.20 & $2.21 \cdot 10^{-5}$ & 5800 \\
H & 0.09 & 2.30 & $1.67 \cdot 10^{-5}$ & 6000 \\
\hline
\end{tabular}

Table 2-23: $\quad$ Parameter estimates and standard deviations using the derived mechanistic kinetic model.

\begin{tabular}{lll}
\hline Parameter & Unit & \multicolumn{1}{l}{ Estimate } \\
\hline$k_{\text {catf }}$ & $\mathrm{s}^{-1}$ & $36.139 \pm 0.975$ \\
$K_{e q}$ & $\mathrm{mM}^{-1}$ & $1.441 \pm 0.009$ \\
$K_{m A}$ & $\mathrm{mM}$ & $0.009 \pm 0.115$ \\
$K_{m B}$ & $\mathrm{mM}$ & $0.084 \pm 0.174$ \\
$K_{m P}$ & $\mathrm{mM}$ & $0.002 \pm 0.027$ \\
$K_{i A}$ & $\mathrm{mM}$ & $0.043 \pm 0.657$ \\
\hline
\end{tabular}

Similarly to Section 2.3.3, the mechanistic kinetic model (Eqs. 2-5 and 2-14) is able to reproduce the experimental data accurately (data not shown). However, some parameters are not identifiable (Tables 2-23 and 2-24) as it is the case in the presence of DMSO. 
Table 2-24: Correlation matrix of the estimated parameters using the derived mechanistic kinetic model.

\begin{tabular}{lllllll}
\hline & $K_{\text {catf }}$ & $K_{\text {eq }}$ & $K_{i A}$ & $K_{m A}$ & $K_{m B}$ & $K_{m P}$ \\
\hline$K_{\text {catf }}$ & 1.00 & 0.08 & -0.02 & 0.00 & 0.11 & -0.01 \\
$K_{\text {eq }}$ & & 1.00 & -0.09 & -0.07 & 0.19 & -0.07 \\
$K_{i A}$ & & & 1.00 & 1.00 & -0.96 & 1.00 \\
$K_{m A}$ & & & & 1.00 & -0.94 & 1.00 \\
$K_{m B}$ & & & & & 1.00 & -0.95 \\
$K_{m P}$ & & & & & & 1.00 \\
\hline
\end{tabular}

Thus, the mechanistic model was simplified according to Section 2.2.3.3 and fitted to the same experimental data. The obtained fit (Figure 2-20) is identical to the one using the mechanistic kinetic model. In contrast to the mechanistic model, the remaining four kinetic parameters of the simplified model can be estimated with sufficient precision (Tables 2-25 and 2-26).

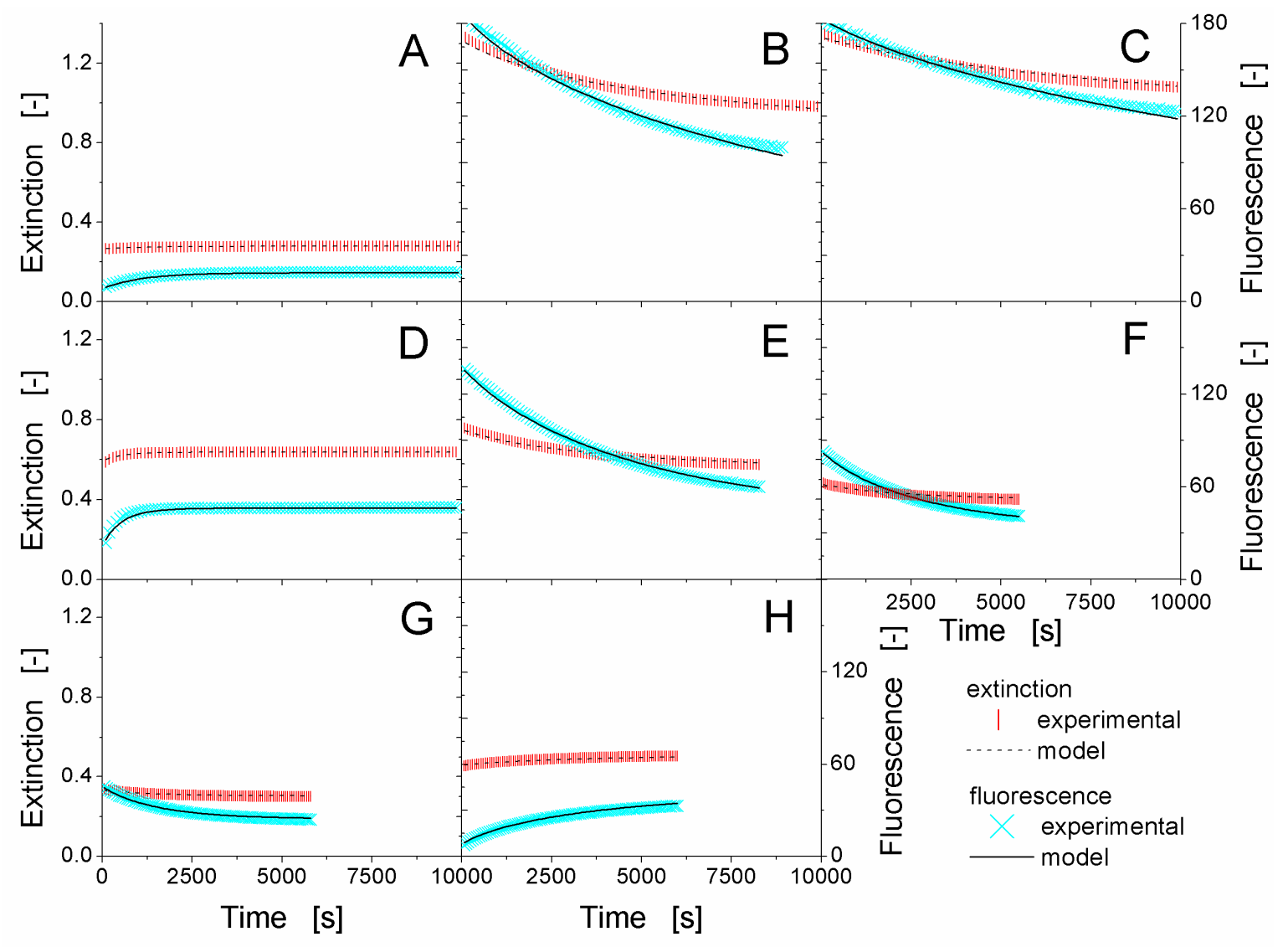

Figure 2-20: Comparison of the fitted simplified kinetic model with the experimental data using experiments A-H (Table 2-22). The model was simultaneously fitted to extinction (red) and fluorescence (blue) intensities. 
Table 2-25: $\quad$ Parameter estimates and standard deviations using the simplified kinetic model.

\begin{tabular}{llr}
\hline Parameter & Unit & \multicolumn{1}{l}{ Estimate } \\
\hline$K_{\text {catf }}$ & $\mathrm{s}^{-1}$ & $40.366 \pm 0.980$ \\
$K_{e q}$ & $\mathrm{mM}^{-1}$ & $1.464 \pm 0.008$ \\
$K_{m A}$ & $\mathrm{mM}$ & $0.147 \pm 0.009$ \\
$K_{m B}$ & $\mathrm{mM}$ & $0.269 \pm 0.014$ \\
\hline
\end{tabular}

Table 2-26: Correlation matrix of the estimated parameters using the simplified kinetic model.

\begin{tabular}{lllll}
\hline & $K_{\text {catf }}$ & $K_{e q}$ & $K_{m A}$ & $K_{m B}$ \\
\hline$K_{\text {catf }}$ & 1.00 & -0.28 & -0.28 & -0.43 \\
$K_{e q}$ & & 1.00 & -0.23 & -0.18 \\
$K_{m A}$ & & & 1.00 & 0.99 \\
$K_{m B}$ & & & & 1.00 \\
\hline
\end{tabular}

As described in Section 2.3, the micro-reaction rate constants and their standard deviations can be estimated by using a micro-kinetic model instead of using error propagation for correlated parameters (Eq. 2-26). The calculated values are listed in Table 2-27. As observed in Section 2.2.3 and also for benzaldehyde in Section 2.7, again the product-release is the rate-limiting step for the carboligation. Obviously, this reaction step is not affected significantly by the change of the cosolvent, while the micro-reaction rate constants for all other steps are significantly lower in DMF than in DMSO (Table 2-15).

Table 2-27: $\quad$ Estimated micro-reaction rate constants with their standard deviations.

\begin{tabular}{lccl}
\hline Micro-reaction rate constant & Calculated value & Standard deviation & Unit \\
\hline$k_{1}, k_{2}$ & 275.4 & 19.0 & $\mathrm{mM}^{-1} \mathrm{~s}^{-1}$ \\
$k_{-1}, k_{-2}$ & 33.8 & 0.9 & $\mathrm{~s}^{-1}$ \\
$k_{3}$ & 40.4 & 1.0 & $\mathrm{~s}^{-1}$ \\
$k_{-3}$ & 1826.8 & 189.2 & $\mathrm{mM}^{-1} \mathrm{~s}^{-1}$ \\
\hline
\end{tabular}




\subsubsection{Conclusions}

The change of the cosolvent from DMSO to DMF increases the solubilities of the reactants. These higher solubilities and the availability of enantiopure product increase the design space for OED. However, kinetic measurements in the presence of DMF require both fluorescence and extinction measurements as well as sophisticated calibration models. As observed previously in Section 2.2, only the introduction of the mechanistic assumption of Section 2.2.3.3 makes all kinetic parameters identifiable. The estimated micro-reaction rate constants indicate that the product release is rate-limiting also in the presence of DMF.

\subsection{Further aspects concerning the kinetic analysis of benzaldehyde lyase}

\subsubsection{Influence of temperature, $\mathrm{pH}$ value, ionic strength, and cosolvent concentration}

The experiments used for model derivation in Section 2.2 were conducted with a constant temperature of $298 \mathrm{~K}$ at $\mathrm{pH}$ 8.5. The $\mathrm{KH}_{2} \mathrm{PO}_{4} / \mathrm{K}_{2} \mathrm{HPO}_{4}$-buffer had an ionic strength of $150 \mathrm{mM}$ and contained 30\%(v/v) DMSO as cosolvent. As already shown for the change of the cosolvent (Section 2.5), the model parameters can be affected by different conditions. Therefore, the influences of temperature $T, \mathrm{pH}$ value, ionic strength $I$, and cosolvent content $c_{\text {cosolvent }}$ were investigated.

Significant influences on model parameters can be observed for $k_{\text {catf }}$ and $K_{\text {eq }}$. Therefore, these parameters are expressed as functions of the influencing factors (Eq. 2-35-36). The functions were related to the reference conditions $T_{r e f}, p H_{r e f}, c_{\text {cosolvent,ref, }}$ and $I_{\text {ref. }}$. The procedure for deriving these equations are described in Schmidt (2008). 


$$
\begin{gathered}
k_{\text {catf }}=k_{\text {catt }, \text { ref }} \cdot \frac{e^{\left.\left(-\frac{E_{\mathrm{a} 1} \cdot\left(\frac{1}{R}-\frac{1}{T}\right)}{T_{\text {ref }}}\right)\right)}}{1+K_{T} \cdot e^{\left(-\frac{E a_{2}}{R}\left(\frac{1}{T}-\frac{1}{T_{\text {ref }}}\right)\right)} \cdot \frac{1+\frac{10^{-p H_{\text {ref }}}}{K_{p H_{1}}}+\frac{K_{p H_{2}}}{10^{-p H_{\text {ref }}}}}{1+\frac{10^{-p H}}{K_{p H_{1}}}+\frac{K_{p H_{2}}}{10^{-p H}}} \cdot} \\
\cdot e^{K_{\text {cosolvent }} \cdot\left(c_{\text {cosolvent }}-c_{\text {cosolent }, \text { ref }}\right)} \cdot \frac{1-\frac{[I]}{K_{\text {lon }}+[I]}}{1-\frac{\left[I_{\text {ref }}\right]}{K_{\text {lon }}+\left[I_{\text {ref }}\right]}} \\
K_{\text {eq }}=K_{\text {eq, ref }} \cdot \exp \left(-\frac{\Delta_{R} H^{\Theta}}{R_{m}}\left(\frac{1}{T}-\frac{1}{T_{\text {ref }}}\right)\right)
\end{gathered}
$$

\subsubsection{Active site titration for benzaldehyde lyase}

BAL has four active sites and is a homo-tetramer of four identical subunits with a molar mass of 59,800 Da including the His-tag (Janzen et al., 2006; Mosbacher et al., 2005). In this chapter it is assumed for all investigations concerning BAL that the added enzyme lyophilisate contains pure BAL without any other proteins. Moreover, it is assumed that all active sites of each enzyme molecule are active. Thus, the molar concentration of active sites can be calculated by dividing the weighted lyophilisate by the molecular weight of the subunit. However, this assumption does not have to be valid. For this reason, an active site titration can be applied which uses suicide substrates, which block the active site. The principle of an active site titration is that the molar concentration of the suicide substrate necessary to inhibit the enzyme completely corresponds to the molar concentration of active sites.

For penicillin amidase Svedas et al. (1977) developed an active site titration using phenylmethylsulfonyl fluoride, which was used in Section 2.1. However, no active site titration for BAL is known so far. Therefore, Dr. Lasse Greiner (ITMC, RWTH Aachen University) proposed that benzaldehyde derivatives which are substituted with halogens like bromine could be promising candidates for suicide substrates since they possibly form nonreactive intermediates with the cofactor thiamine diphosphate. To check this, 4-bromomethyl- 
benzaldehyde (Figure 2-21) was synthesized by Dr. Sanjib Kumar Karmee (ITMC, RWTH Aachen University) and first experiments revealed a minor inhibition of BAL. However, no stoichiometric inhibition could be observed yet. Further experiments should focus on the investigation of the incubation time, the medium composition and the cofactor excess.

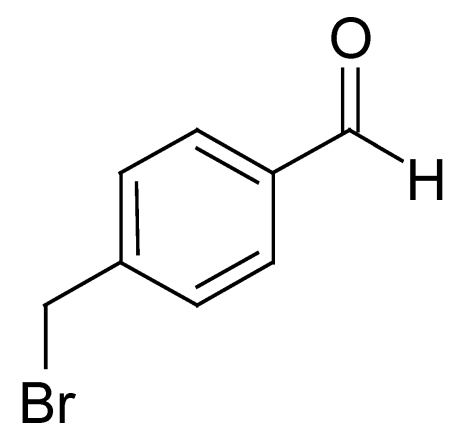

Figure 2-21: 4-Bromomethyl-benzaldehyde - a possible suicide substrate for BAL.

\subsection{Investigating the carboligase activity of thiamin diphosphate-dependent enzymes using mechanistic kinetic modeling and NMR analysis}

\subsubsection{Introduction}

Thiamin diphosphate (ThDP)-dependent enzymes catalyze carboligase reactions with an enantiomeric excess above 99\% (Demir et al., 2001b; Iding et al., 2000; Pohl et al., 2002). The kinetics of one of these enzymes - benzaldehyde lyase (BAL, EC 4.1.2.38) - is comprehensively studied in Section 2.2. The estimated values for the kinetic parameters of the derived mechanistic kinetic model indicate that the release of the product is the rate-limiting step for the carboligation of two 3,5-dimethoxy-benzaldehyde (DMBA) molecules. To investigate if the release of the product is also rate-limiting for other ThDP-dependent enzymes, the kinetics of BAL are compared with those of benzoylformate decarboxylase from Pseudomonas putida (BFD, EC 4.1.1.7).

BFD was first reported by Wilcocks et al. (1992). The enzyme is involved in the nonoxidative decarboxylation of benzoylformate (Iding et al., 2000), and is able to catalyze the enantioselective synthesis of (S)-2-hydroxypropanone derivatives (Demir et al., 1999). BFD 
further catalyzes the ligation of a broad range of aromatic, heteroaromatic, cyclic conjugated olefinic aldehydes as donor substrates, preferably with acetaldehyde as an acceptor. Besides acetaldehyde, BFD converts aromatic and heteroaromatic substrates as acceptors to produce enantiopure $(R)$-benzoin and derivatives, but in contrast to BAL with lower reaction rates (Iding et al., 2000). BFD basically follows the same mechanism as BAL (Figure 2-7) with the only difference that no cleavage of the formed ( $R$ )-2-hydroxy ketones could be observed so far (Reynolds et al., 1988; Iding et al., 2000; Weiss et al., 1988). The benzoin-forming activity of BFD was enhanced by site-directed mutagenesis of histidine 281 to alanine, yielding more space in the active site for accommodating larger acceptor aldehydes (Demir et al., 1999). Therefore, the variant BFD H281A was chosen for the comparison of the enzyme mechanisms.

As demonstrated in Section 2.3 the quantification of the enzyme intermediates in the steadystate region shows which micro-reaction step is rate-limiting. Thus, besides progress curve analysis steady-state intermediate analysis using ${ }^{1} \mathrm{H}$ nuclear magnetic resonance $\left({ }^{1} \mathrm{H}\right.$ NMR $)$ is applied. The kinetics of BAL and BFD H281A were compared using the carboligation of two unsubstituted benzaldehyde molecules to $(R)$-benzoin (Figure 2-22).<smiles>O=Cc1ccccc1</smiles>
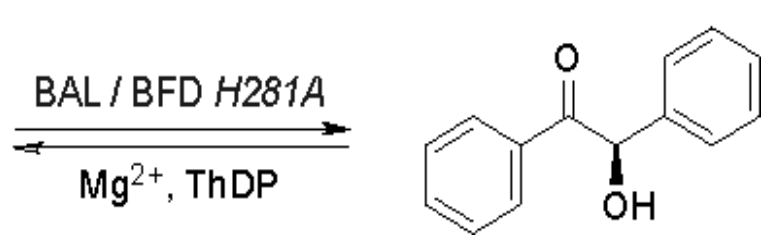

Figure 2-22: $\quad$ Synthesis of two benzaldehyde molecules to $(R)$-benzoin using either BAL or BFD $H 281 A$.

\subsubsection{Materials and methods}

All experiments were conducted by Mariya Kokova (Institute of Molecular Enzyme Technology, Heinrich-Heine University Düsseldorf/Research Centre Jülich). The NMR experiments were performed in cooperation with Professor Kai Tittmann (Institute for Biochemistry, Martin-Luther-University Halle-Wittenberg).

The buffering salts were purchased from Roth (Karlsruhe, Germany), all other reagents and organic solvents including deuterated acetone and DMSO were obtained from Sigma-Aldrich (Deisenhofen, Germany). All chemicals were of analytical purity. The reaction mixture 
consisted of benzaldehyde $(5-60 \mathrm{mM})$ in $\mathrm{KH}_{2} \mathrm{PO}_{4} / \mathrm{K}_{2} \mathrm{HPO}_{4}$ buffer $(50 \mathrm{mM})$, containing $2.5 \mathrm{mM} \mathrm{MgSO} 4,0.5 \mathrm{mM}$ ThDP and BAL $(5-10 \mu \mathrm{g} / \mathrm{ml})$ or BFD H281A (40-60 $\mu \mathrm{g} / \mathrm{ml})$. BAL kinetics were studied at $\mathrm{pH} 8$ and in case of BFD $H 281 A$ at $\mathrm{pH}$ 6.5. The assay temperature for both enzymes was kept at $30^{\circ} \mathrm{C}$. The protein content was determined according to Bradford (1976) using bovine serum albumin as a standard.

For progress curve analysis the benzaldehyde consumption and the benzoin formation were quantified. Samples were taken at appropriate time intervals, diluted in acetonitrile $(1: 20)$ to inactivate the enzyme, centrifuged and subjected to high performance liquid chromatography (HPLC) analysis (Gynkotek, Germering, Germany), equipped with an ODS Multohyp column ( $5 \mu$, CS-Chromatographie, Langerwehe, Germany) and a UV-detector. Up to seven samples were taken in order to follow the reaction within the first five minutes. The obtained data points of both benzaldehyde and benzoin were used to fit the mechanistic kinetic model (Eqs. 2-5 and 2-14).

Using a combined acid quench $/{ }^{1} \mathrm{H}$ NMR-method (Tittmann et al., 2003), the relative distribution of ThDP and of the acid-stable intermediates HBz-ThDP and benzoin-ThDP can

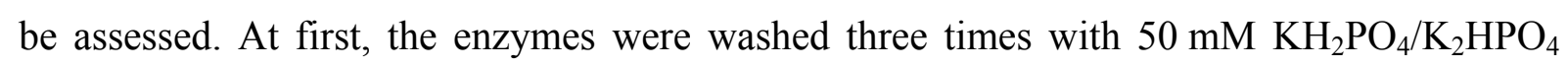
buffer at $4^{\circ} \mathrm{C}$ to remove excess ThDP. Thereafter, BAL $(6 \mathrm{mg} / \mathrm{ml})$ or BFD H281A $(10 \mathrm{mg} / \mathrm{ml})$ were mixed with $20 \mathrm{mM}$ benzaldehyde in $50 \mathrm{mM} \mathrm{KH} \mathrm{PO}_{4} / \mathrm{K}_{2} \mathrm{HPO}_{4}$ buffer ( $\mathrm{pH} 7.5$ for BAL and $\mathrm{pH} 6.5$ for $\mathrm{BFDH} 281 \mathrm{~A}$ ) at $30^{\circ} \mathrm{C}$ for $1-2 \mathrm{~s}$ to assure steady-state conditions. The reactions were stopped by addition of trichloroacetic acid and $\mathrm{HCl}$. After centrifugation the precipitated protein was discarded and the supernatant containing the intermediates, substrates and products of the reaction were subjected to ${ }^{1} \mathrm{H}$ NMR spectroscopy at $298 \mathrm{~K}$ using water presaturation techniques for suppressing the water signal. For assignment and quantitative analysis of ThDP, hydroxybenzyl-ThDP (HBz-ThDP) and benzoin-ThDP, the 2'- $\mathrm{CH}_{3}$ and 4$\mathrm{CH}_{3}{ }^{1} \mathrm{H}$ NMR singlet signals of ThDP (2.65 and $2.58 \mathrm{ppm}$ ), HBz-ThDP (2.47 and $2.42 \mathrm{ppm}$ ) and benzoin-ThDP (2.45 and $2.43 \mathrm{ppm})$ were used.

Due to the low solubility of the benzoin substrates in aqueous buffer, Stillger et al. (2006) recommended the addition of DMSO or acetone. The effects of both cosolvents on the reaction kinetics and mechanism were also analyzed. Activity of BAL was measured in $10 \%(\mathrm{v} / \mathrm{v})$ DMSO and $20 \%(\mathrm{v} / \mathrm{v})$ acetone, activity of BFD $H 281 \mathrm{~A}$ was additionally studied in $20 \%(\mathrm{v} / \mathrm{v})$ DMSO. 


\subsubsection{Results and discussion}

One inherent drawback of the applied HPLC analysis is the low number of data points compared to quasi-continuous measurement techniques such as fluorescence spectroscopy. Thus, the precision of estimated parameters is per se lower (Section 2.4). Moreover, without the presence of any cosolvent the concentration of benzaldehyde cannot be raised above $50 \mathrm{mM}$, because otherwise a second phase is formed. Hence, it is not astonishing that also in this case the mechanistically motivated simplification described in Section 2.2.3.3 has to be introduced to obtain identifiability. Moreover, it is assumed that the thermodynamic equilibrium constant $K_{e q}$ is not affected by the cosolvent.

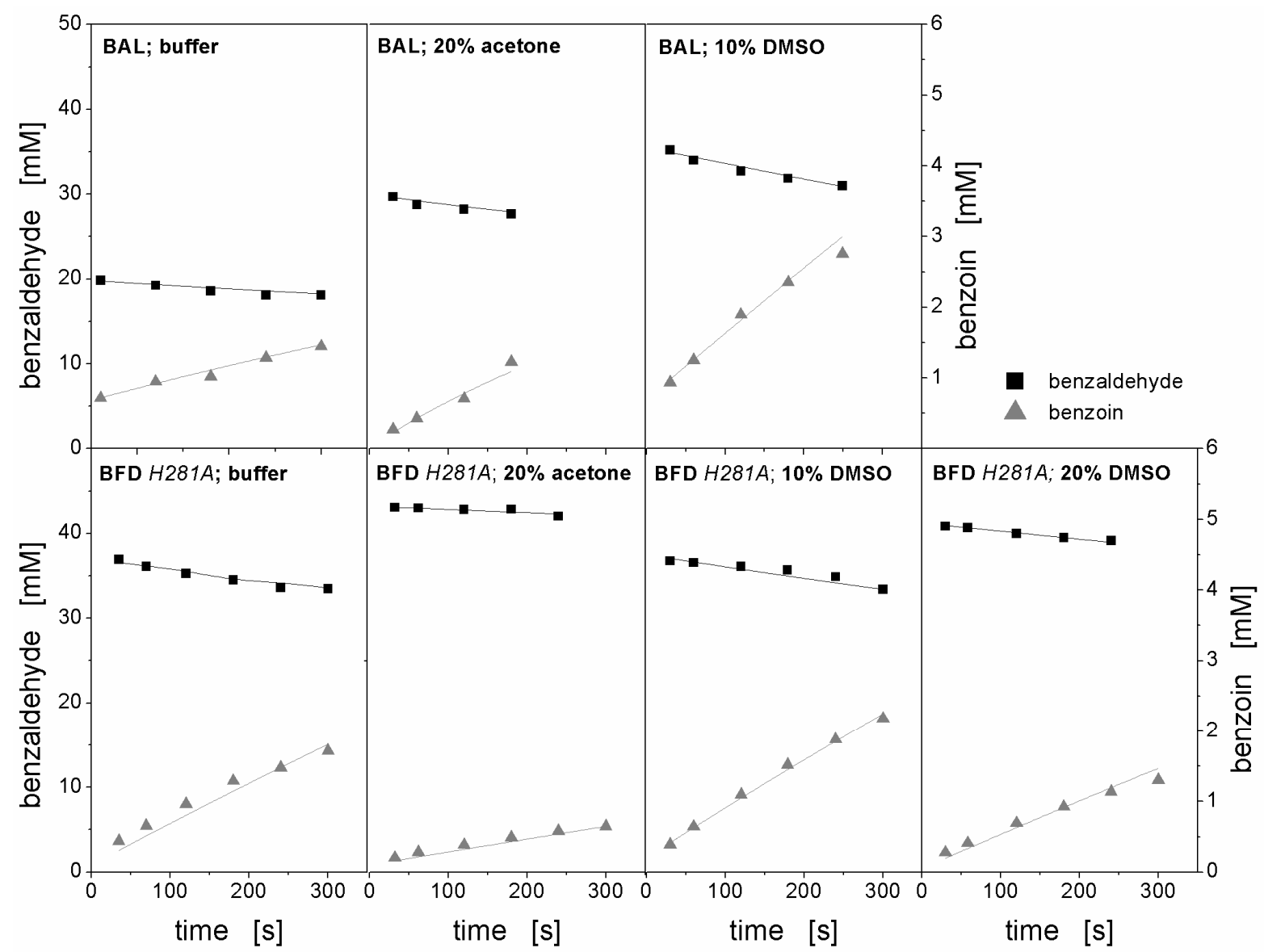

Figure 2-23: Selection of progress curves fitted by the simplified kinetic model. For each tested cosolvent the measured concentrations for benzaldehyde (left axis) and benzoin (right axis) are plotted over time.

Figure 2-23 shows the fit using the simplified kinetic model. The estimated values for the kinetic parameters are listed in Table 2-28. The maximum turnover number $k_{\text {catf }}$ can be 
estimated precisely, the precision for $K_{m A}$ and $K_{m B}$ is satisfactory. The cosolvents affect all kinetic parameters. Both enzymes show a higher $k_{\text {catf }}$ in presence of the cosolvent compared to buffer with only one exception: BFD $H 281 A$ in $20 \%(\mathrm{v} / \mathrm{v})$ acetone. The addition of $10 \%(\mathrm{v} / \mathrm{v})$ DMSO caused the strongest effect on both enzymes.

Table 2-28: $\quad$ Estimated independent parameters and their standard deviations for the formation of benzoin from benzaldehyde in $50 \mathrm{mM} \mathrm{KH_{2 }} \mathrm{PO}_{4} / \mathrm{K}_{2} \mathrm{HPO}_{4}$ buffer with and without cosolvents. $K_{e q}$ was determined separately to $3.02 \pm 0.13 \mathrm{mM}^{-1}$.

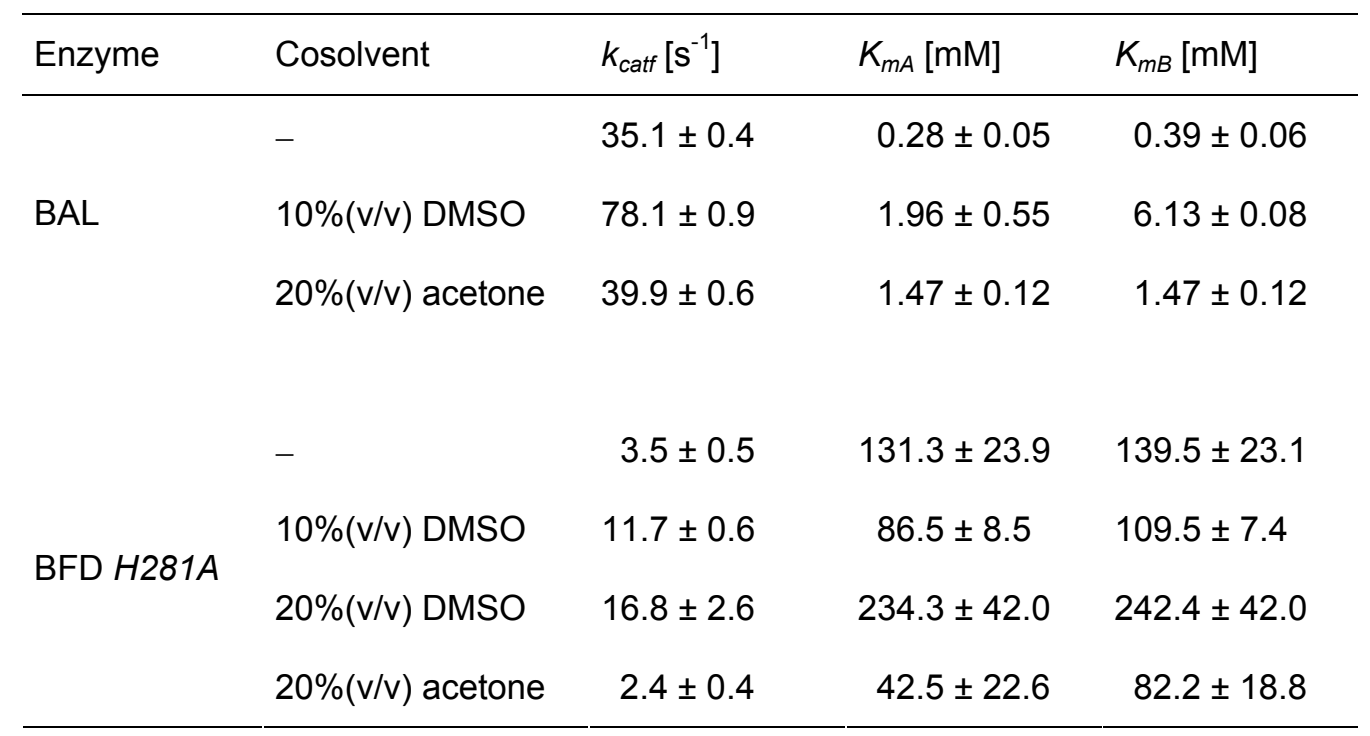

By comparing the values obtained for both enzymes, it becomes apparent that BAL is about one order of magnitude more active than BFD H281A. This is not surprising since BAL is known to be significantly more active concerning benzoin formation (Demir et al., 1999).

Compared to BAL, the $K_{m}$-values of BFD $H 281 A$ are much higher. This property can clearly be seen in Figure 2-24, where the initial reaction rates are plotted versus the initial substrate concentrations. While BAL exhibits the typical hyperbolic shape, the initial reaction rates of BFD $H 281 A$ show an almost linear dependence on the initial substrate concentration within the solubility limits. This extremely low affinity for aliphatic or aromatic aldehyde substrates is probably a specific property of BFD. This behavior was also previously observed for wildtype BFD and propanal as a substrate (Mikolajek et al., 2007, 2009). 

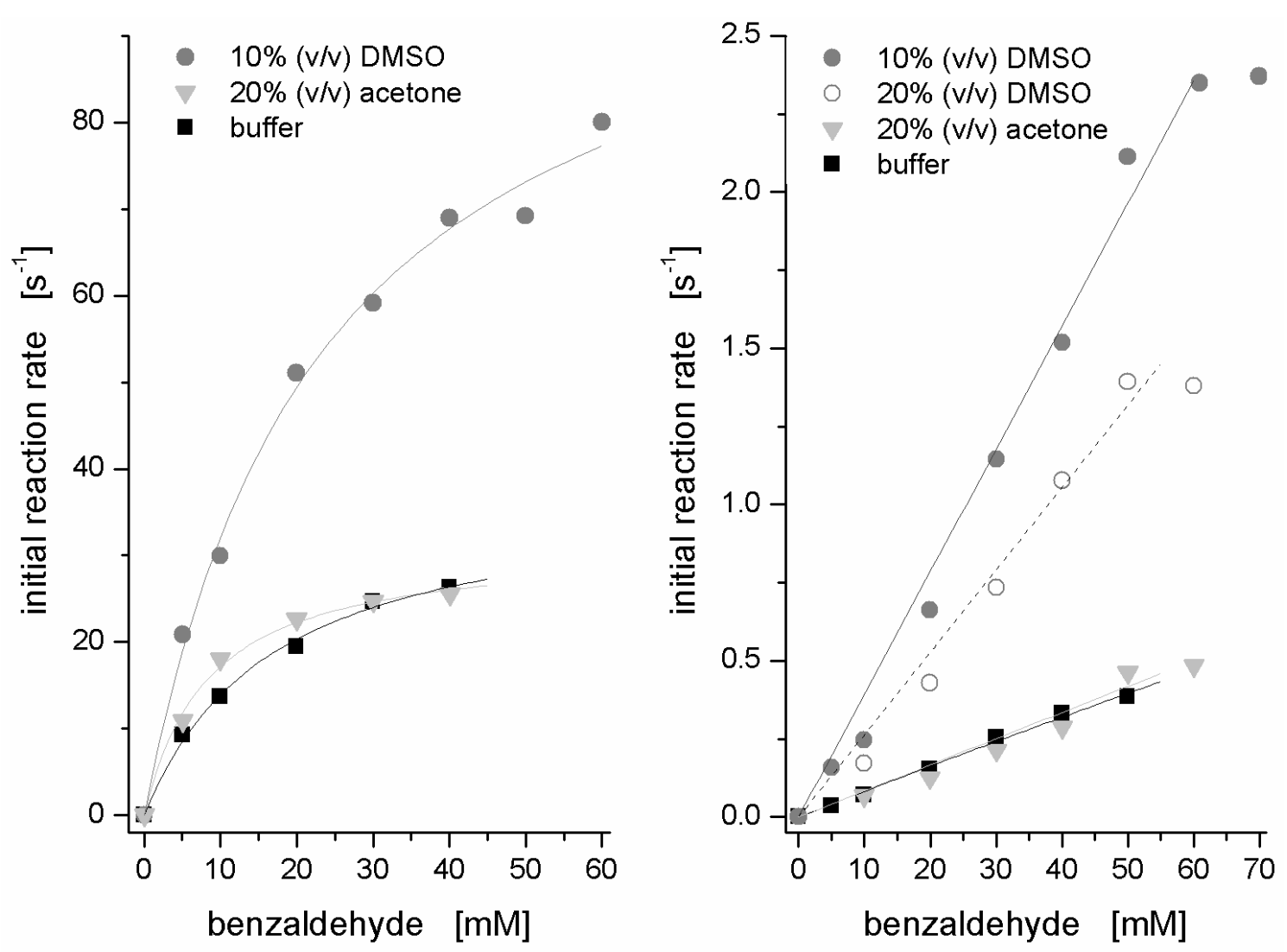

Figure 2-24: Initial reaction rates of BAL (left) and BFD H281A (right) in presence of different cosolvents.

The micro-reaction rate constants were calculated according to Eqs. 2-20-23. In Figure 2-25 the calculated values are not only presented as numbers, but are also visualized in terms of a bar chart. Thus, it can clearly be seen that for BAL and BFD $H 281 A$ different kinds of kinetic limitations exist. Catalysis using BAL is mostly rate-determined by the release of the product even for the unsubstituted benzaldehyde molecules. Moreover, the bar charts visualize the kinetic effect of the cosolvents: they accelerate the release of benzoin, while they decrease the substrate binding rate. The overall reaction rate is increased since the product-release is the bottleneck. Taking into account that $k_{1}$ and $k_{2}$ are second order rate constants, it can be concluded that above $0.28 \mathrm{mM}$ benzaldehyde in buffer, above $1.96 \mathrm{mM}$ in $10 \%(\mathrm{v} / \mathrm{v}) \mathrm{DMSO}$, and above $1.32 \mathrm{mM}$ in $20 \%(\mathrm{v} / \mathrm{v})$ acetone the product-release is rate-limiting. These results are in line with the results from Section 2.2 for the synthesis of $(R)-3,3$ ',5,5'-tetramethoxybenzoin. Moreover, in a recent publication spectroscopic data based on circular dichroism indicates that the release of benzoin could be rate-limiting for BAL-catalyzed benzoin synthesis (Chakraborty et al., 2008). Conversely to BAL, Figure 2-25 shows that the release of the product is the fastest reaction step for the benzoin formation catalyzed by BFD $H 281 A$, whereas the carboligation is the rate-limiting step. The carboligation also remains rate- 
limiting in the presence of cosolvents. Moreover, the results implicate DMSO to be a better cosolvent than acetone, as all micro-reaction rate constants are higher in DMSO.

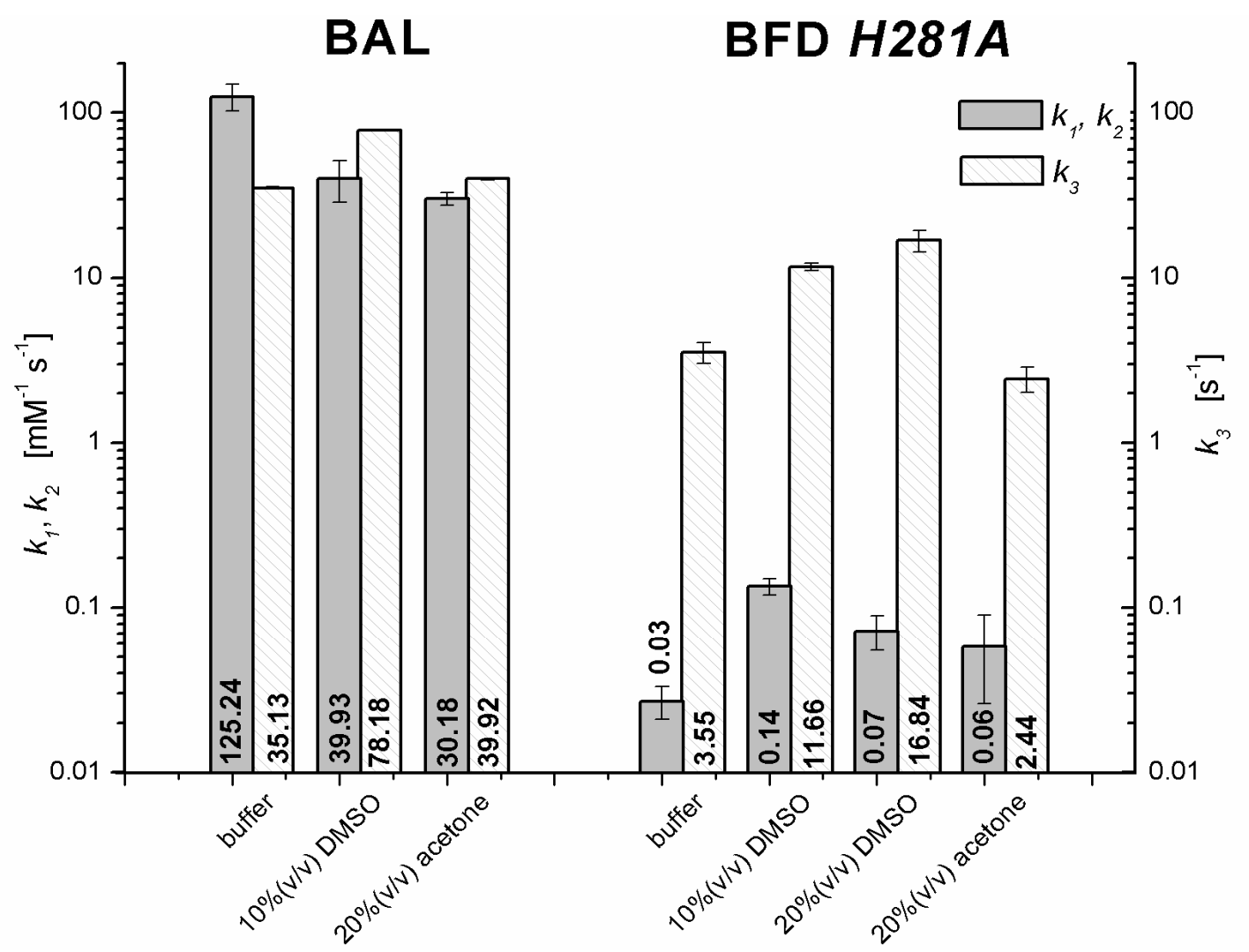

Figure 2-25: Calculated micro-reaction rate constants using the simplified kinetic model.

The different rate limitations with BAL and BFD $H 281 \mathrm{~A}$ can be rationalized in terms of different active site geometries. Structural studies from Knoll et al. (2006) show that the active site of BAL is partly covered by a C-terminal helix, whereas in BFD $H 281 \mathrm{~A}$ such a structural element is absent (Figure 2-26).

Figure 2-27 illustrates the difference between both enzymes regarding the release of the product. In BAL the C-terminal helix forms a bottleneck which hinders the release of the formed product from the active site. Thus, the product stays longer within the active site and can even rebind to the cofactor ThDP. For this reason, the product-release is rate-limiting. In BFD $H 281 A$ this step is not rate-limiting since no bottleneck exists and the product can easily leave the active site. Also other microscopic explanations for these different behaviors of both enzymes are conceivable, but the different active site geometries are the most distinct difference between both enzymes. 

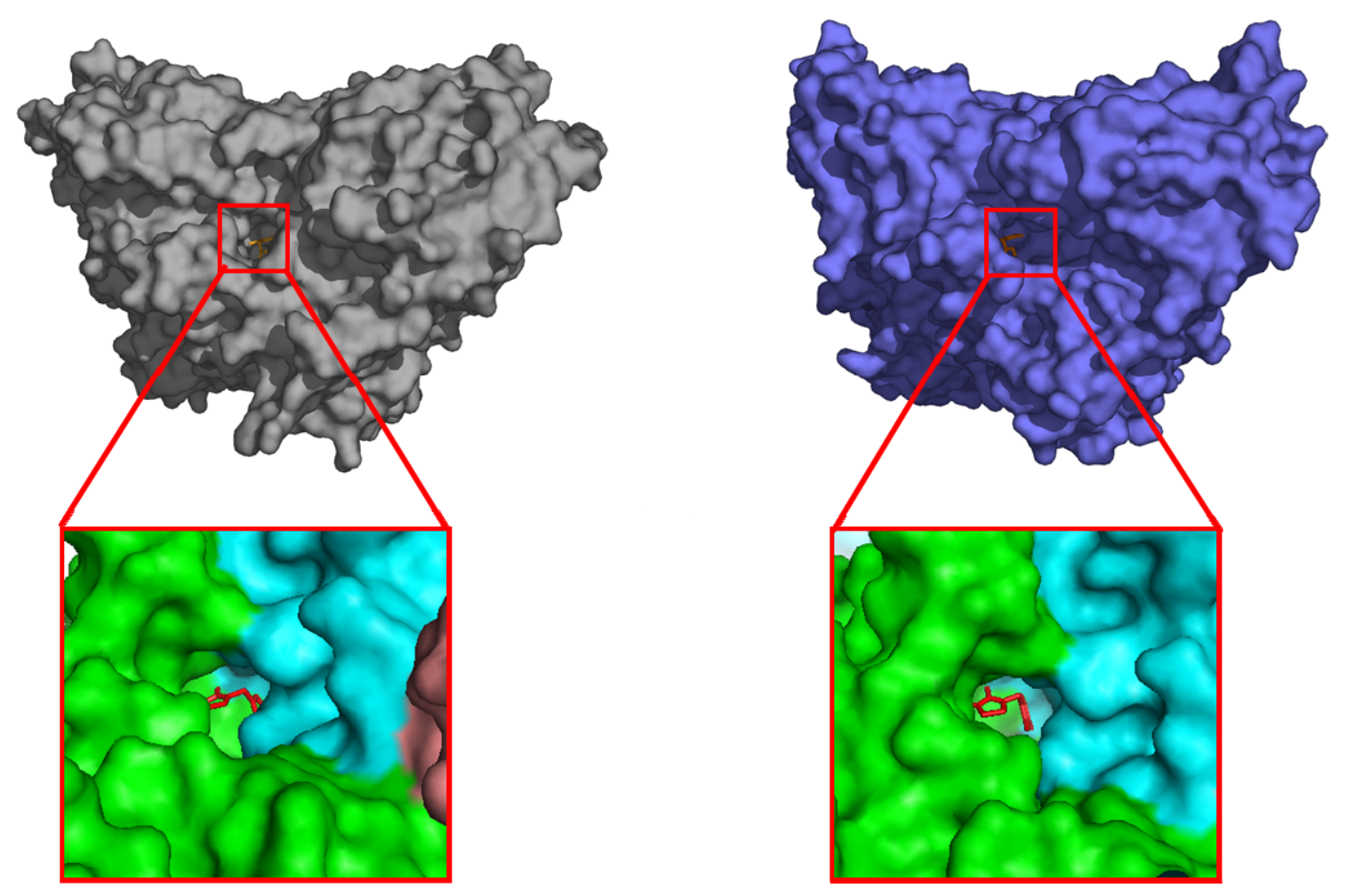

Figure 2-26: Crystal structure of BAL (left) and BFD H281A (right). The active sites are enlarged. The pictures are adapted from the RCSB Protein Data Bank entries 2ag0 (BAL) and 1mcz (BFD H281A) which were generated by Dr. Michael Knoll, Institute of Technical Biochemistry, University of Stuttgart.
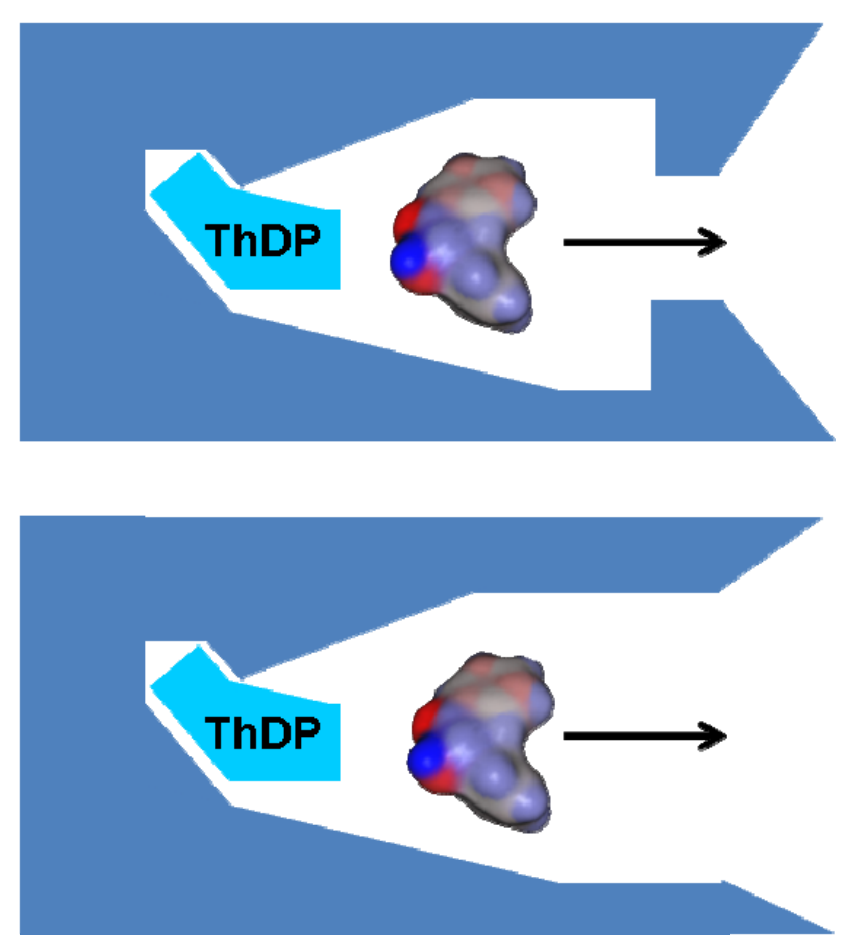

Figure 2-27: Scheme for the cross section of the active sites for BAL (top) and BFD H281A (bottom) adapted from Knoll et al. (2006). 
As shown in Section 2.3 rate-limiting steps can also be identified by steady-state intermediate analysis. The results using ${ }^{1} \mathrm{H}$ NMR spectroscopy are shown in Figure 2-28. These results provide only qualitative information since it was observed that BAL rapidly loses the cofactor ThDP from the active sites when dissolved in buffer without excess cofactors. The NMR analysis cannot distinguish between free and enzyme-bound ThDP thus making a quantitative estimation of enzyme-bound C2-unsubstituted ThDP not reliable. Moreover, no saturation of BFD $H 281 A$ can be achieved due to the very high $K_{m}$-values. Therefore, only the peaks of the first intermediate (EA), HBz-ThDP, and of the second intermediate (EAA), benzoin-ThDP, should be taken into account.
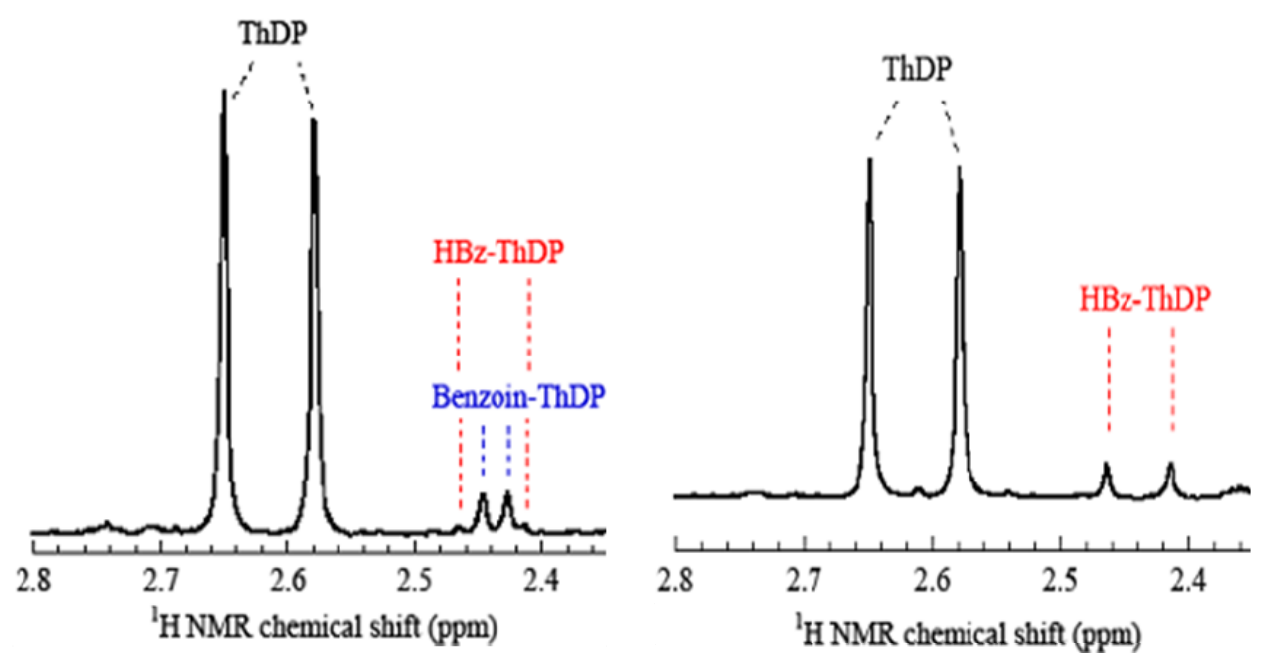

Figure 2-28: Distribution of reaction intermediates from steady-state intermediate analysis by ${ }^{1} \mathrm{H} N M R$. The $2^{\prime}-\mathrm{CH}_{3}$ and the 4- $\mathrm{CH}_{3}$ fingerprint region is shown for BAL (left) and BFD H281A (right) in aqueous buffer.

If the results for BAL and BFD $H 281 A$ are compared it is striking that different intermediates are accumulated. If BAL is used, the second intermediate (benzoin-ThDP) accumulates because the subsequent step, the release of the product, is rate-limiting. In contrast to this, only the first intermediate (HBz-ThDP) can be detected using BFD H281A, which indicates that the carboligation is rate-limiting. Here, the release of the product is fast as no benzoinThDP adduct can be observed. Once the benzoin-ThDP is formed, the product will immediately split off from ThDP and leave the active site. In presence of cosolvents, similar results are obtained (results not shown). Thus, these results are in line with those from progress curve analysis and support the possible microscopic explanation. 


\subsubsection{Conclusions}

In this Section the kinetics of BAL and the BFD variant $H 281 A$ were analyzed and compared by conducting progress curve analysis. Using the simplified kinetic model derived in Section 2.2 it is possible to determine the micro-reaction rate constants and the rate-limiting steps for the benzoin condensation. The model-based experimental analysis revealed that BAL is mainly limited by the release of the product, which is consistent with the results from Section 2.2. For BFD $H 281 A$, the rate-limiting step was identified to be the carboligation of $\mathrm{HBz}-$ ThDP and benzaldehyde. These different limitations are probably caused by different geometries of the active sites of both enzymes. The results are qualitatively supported by independent ${ }^{1} \mathrm{H}$ NMR analysis of steady-state intermediates.

According to the proposed microscopic explanation, the channel to the active site of BAL exhibits a narrow bottleneck which hinders the release of the formed product into the bulk. Therefore, this bottleneck should be broadened. This can be achieved by changing or eliminating amino acids of the protein structure. Thus, three variants of BAL were developed by Melanie Schwarz (Institute for Molecular Enzyme Technology, Heinrich-Heine-University Düsseldorf/Research Centre Jülich) using a rational design approach. These BAL variants should exhibit active site geometries similar to Figure 2-27 (bottom) and are currently tested for activity. An enhanced activity of one of these variants would prove that mechanistic kinetic modeling of enzyme kinetics can lead to improved enzymes. 


\section{Mass Transfer Between Two Liquid Phases}

In biocatalysis, monophasic reaction systems are often used because they offer general advantages such as fast reaction rates and high selectivities. Unfortunately, this is inherently linked with the difficulty of separating the biocatalysts from the reactive mixture which strongly impacts economics. Moreover, it is shown in the previous chapter for the symmetric carboligation of two 3,5-dimethoxy-benzaldehyde (DMBA) molecules using benzaldehyde lyase (BAL) that only low concentrations of the product $(R)-3,3$ ',5,5'-tetramethoxy-benzoin (TMB) can be achieved in monophasic aqueous systems. A promising strategy to push the approach of biocatalysis to an industrial scale is the utilization of biphasic reaction systems.

A biphasic reaction system consists of two phases, a reactive phase, in which the biocatalyst, e.g. an enzyme, is dissolved and the reaction takes place, and a non-reactive phase acting as substrate reservoir and in situ extraction solvent (Halling, 1994). The reactive phase is usually aqueous since most biocatalysts show their highest activities in aqueous media, while for the non-reactive phase a broad range of solvents can be chosen. However, this solvent choice is often empirically performed which requires the experimental screening of large amounts of possible solvents. Therefore, in this chapter a systematic procedure is proposed which bases on a combination of ab initio calculations using the Conductor-like Screening Model for Realistic Solvation (COSMO-RS), guided experiments, and dynamic modeling. Thus, both thermodynamic and, afterwards, kinetic properties are considered.

\subsection{Systematic approach for solvent selection}

In a biphasic reaction system, the reactants show a certain affinity to each of the two phases, expressed by their partition coefficients (Eq. 3-1), where $P_{i}$ is the partition coefficient of compound $i$ and $c_{i, o r g}$ and $c_{i, a q}$ are the concentrations of the compound in the organic and aqueous phase, respectively.

$$
P_{i}=\frac{c_{i, \text { org }}(t \rightarrow \infty)}{c_{i, a q}(t \rightarrow \infty)}
$$


The solvent choice for the non-reactive phase determines the values of the partition coefficients and thus the equilibrium concentrations, which in turn affect the maximum yield which can be reached. The yield is defined as the ratio of the amount of substrate, which reacted to the desired product, to the initial substrate amount $n_{A, 0}$. In biphasic reaction systems the product is harvested from the non-reactive phase. Thus, only the product present in the non-reactive phase is taken into account. Accordingly, the maximum extractable yield $Y$ can be calculated with Eq. 3-2, where $n_{P, \text { org }}$ denotes the amount of product in the organic phase.

$$
Y=\frac{2 \cdot n_{P, o r g}(t \rightarrow \infty)}{n_{A, 0}}
$$

Figure 3-1 shows the dependence of the maximum extractable yield on the partition coefficients. Obviously, a high yield can be achieved with a solvent which exhibits a large product partition coefficient $P_{P}$, whereas the substrate partition coefficient $P_{A}$ should be as low as possible.

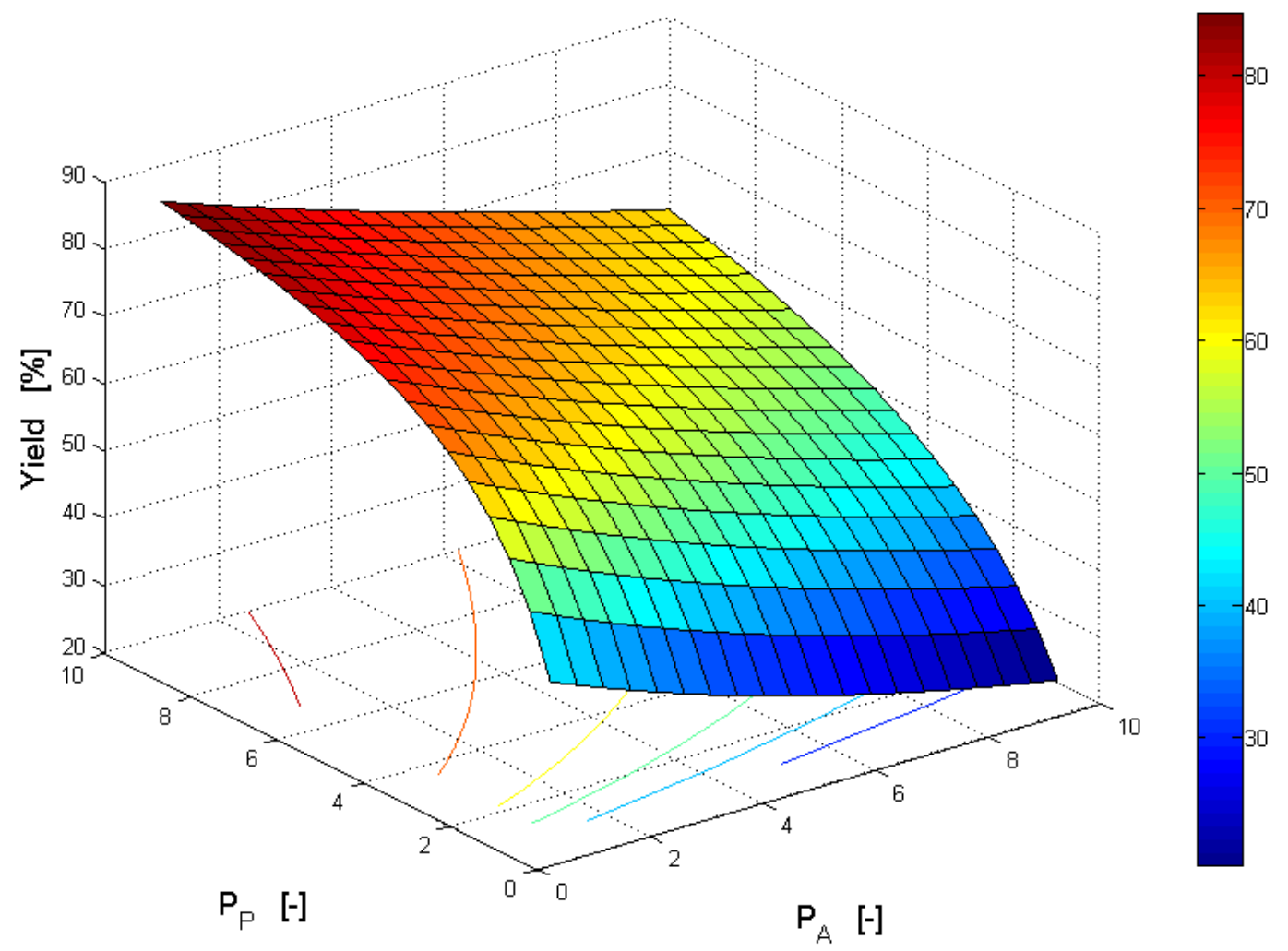

Figure 3-1: $\quad$ Maximum extractable yield for the symmetric carboligation in a biphasic reaction system dependent on the partition coefficients $\left(V_{o r g} / V_{a q}=1 ; c_{A, 0}=10 \mathrm{mM} ; K_{e q}=1.464\right)$. 
From a thermodynamic point of view, this means that a well-suited solvent will continuously provide the substrate to the reactive phase, while it extracts the product from the reactive to the non-reactive phase. Thus, the thermodynamic equilibrium is shifted towards the product side, which increases the yield. Consequently, a factor $R$ is introduced (Eq. 3-3), defined as the ratio of the product partition coefficient divided by the substrate partition coefficient. This factor describes the ability of the solvent in the non-reactive phase to "push" the substrate into the reactive phase and to "pull" the product out of the reactive phase.

$$
R=\frac{P_{P}}{P_{A}}
$$

By increasing $R$, the maximum extractable yield is maximized. However, a general problem of various reactions of preparative and industrial interest is their disadvantageous $R$. In order to effectively design biphasic reaction systems, a distinct knowledge of $R$ is required. To obtain reliable data for $R$, a large number of time-consuming and expensive experiments is necessary. To overcome the effort of numerous experiments for reaction optimization, a systematic approach to an optimal solvent selection for biphasic systems is proposed. This systematic approach is illustrated in Figure 3-2.

The systematic approach starts with quantum mechanical geometry optimizations and COSMO calculations. Within the subsequent COSMO-RS calculations there are two selection steps that the solvents need to pass. The first selection step is the calculation of liquid-liquid equilibria (LLE). If the solvents do not show a miscibility gap with water at reaction temperature, they are eliminated in the first step. Those solvents that actually show a miscibility gap with water enter into the second selection step, the calculation of partition coefficients. From the partition coefficients $R$ is calculated. A well-suited solvent is characterized by a large value for $R$. Hence, the solvents can be ranked accordingly.

COSMO-RS allows a fast and effective solvent screening via partition coefficients at infinite dilution. However, this method can only predict thermodynamic properties at equilibrium, but no kinetic behavior. Moreover, COSMO-RS predicts general trends for partition coefficients, but no absolute values (Eckstein et al., 2006b; Spiess et al., 2008a). 
To verify the COSMO-RS solvent ranking and to investigate mass transfer kinetics, kinetic modeling using dynamic modeling software like gPROMS is a valuable tool. With this method, mass transfer experiments can be designed in order to obtain precise values for the mass transfer coefficients and partition coefficients. Furthermore, the dependency of the coefficients on process parameters such as temperature, $\mathrm{pH}$, ionic strength, and cosolvent can be determined. The method depends on experimental data to fit the parameters. Thus, it should not be used for solvent screening without preceding COSMO-RS calculations since the necessary experimentation is laborious and time-consuming. But since for this last step only the most promising solvents are chosen, the experimental effort is minimized and more reliable and detailed results are obtained.

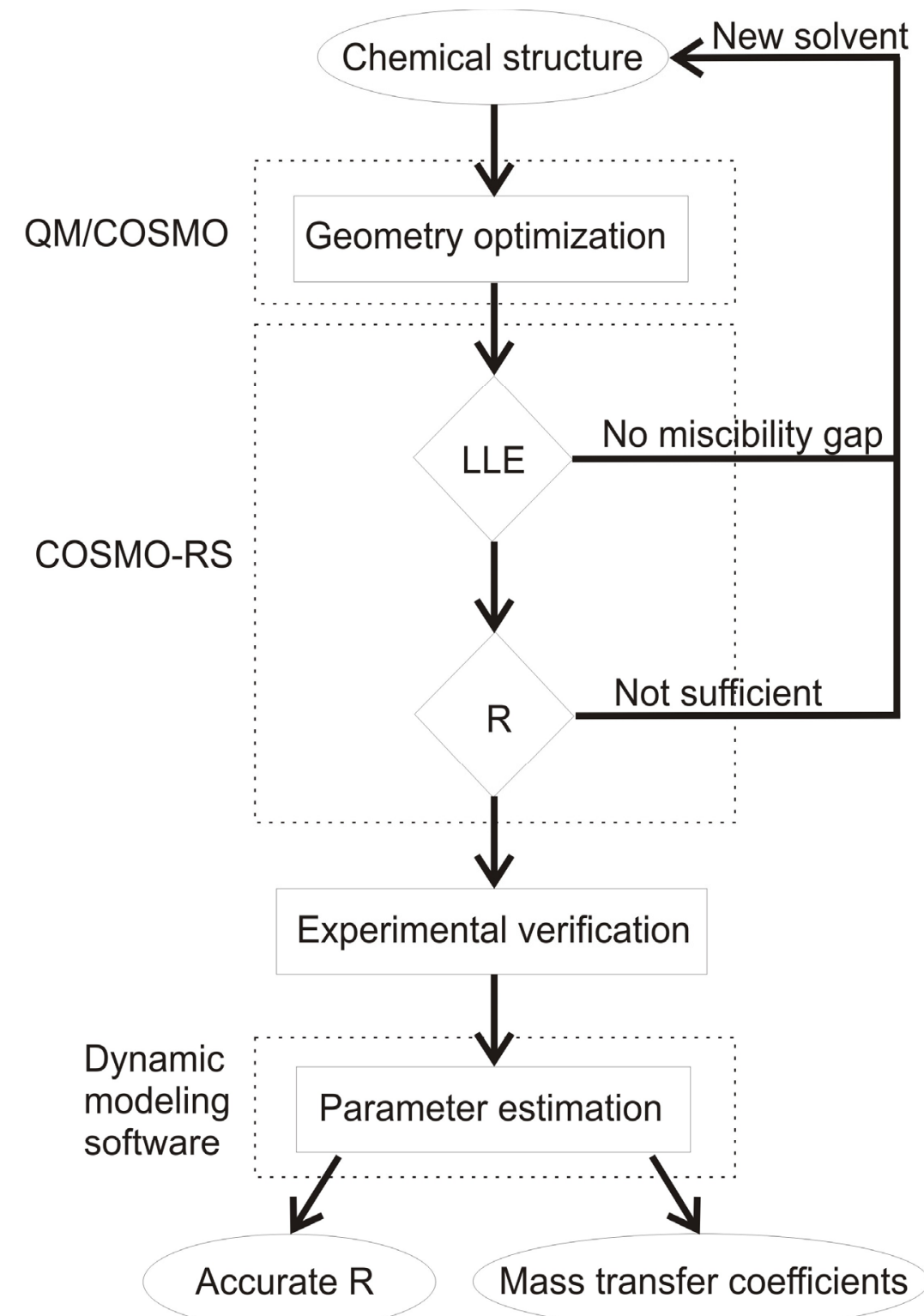

Figure 3-2: $\quad$ The proposed systematic approach for solvent selection. 


\subsection{Materials and methods}

\subsubsection{COSMO-RS}

The ab initio calculations were conducted by Martina Peters (ITMC, RWTH Aachen

University). Quantum mechanical geometry optimizations were performed using TURBOMOLE (Version 5.7.1, COSMOlogic, Leverkusen, Germany) (Ahlrichs et al., 1989; Häser and Ahlrichs, 1989; Horn et al., 1991; Klamt and Schüürmann, 1993; Treutler and Ahrichs, 1995). The statistical thermodynamics calculations were performed using the COSMOtherm software (Version C2.1-0106, COSMOlogic, Leverkusen, Germany) (Eckert, 2006). Subsequent to that, COSMO-RS was used as a bridge from single molecule properties like screening charge densities to macroscopic properties of pure systems and mixtures. It is based on assumptions on the interaction between the charged surface segments. To calculate partition coefficients at infinite dilution, Eq. 3-4 was used according to Eckert (2006), where $\mu_{i j}$ is the chemical potential of compound $i$ in solvent $j$ and $v_{j}$ is the molar volume of solvent $j$.

$$
\log P_{i}=\log \left(\exp \left[\frac{\mu_{i, 1}-\mu_{i, 2}}{R_{m} T}\right] \frac{v_{1}}{v_{2}}\right)
$$

\subsubsection{Experimental}

All chemical substances were of analytical grade and purchased from Sigma-Aldrich (Deisenhofen, Germany). 3,5-dimethoxy-benzaldehyde (DMBA) was used as substrate. The product $(R)$-3,3',5,5'-tetramethoxy-benzoin (TMB) was synthesized using benzaldehyde lyase (BAL, EC 4.1.2.38) from Pseudomonas fluorescens. For the mass transfer experiments the aqueous phase consisted of a $50 \mathrm{mM} \quad \mathrm{KH}_{2} \mathrm{PO}_{4} / \mathrm{K}_{2} \mathrm{HPO}_{4}$ buffer and $25 \%(\mathrm{v} / \mathrm{v})$ dimethylformamide (DMF). The cosolvent DMF was used to increase the solubility of the aromatic compounds. The $\mathrm{pH}$ value was adjusted to 8.5 and the ionic strength to $100 \mathrm{mM}$. For the organic phase $n$-hexane and methyl-iso-butyl-ketone (MIBK) were used, respectively.

The mass transfer experiments were performed at $25^{\circ} \mathrm{C}$ in a continuously stirred tank reactor which is similar to a Lewis cell. Both phases were stirred individually using two independent stirrers, one from above, one from below. The phases are considered as ideally mixed. The volumes of both phases were equal. As in a Lewis cell, the interface is flat and horizontal and 
its size remains constant. The dynamic measurements were conducted using a spectrophotometer (LS55, Perkin Elmer, Waltheim, USA) which recorded the extinction in the aqueous or the organic phase. The influences of temperature and DMF volume fraction on the partition coefficients were analyzed by taking samples after 24 hours from the aqueous phase. The concentrations were analyzed by high performance liquid chromatography (HPLC) (System Gold, Beckmann Coulter, Munich, Germany).

\subsection{Model development}

For the purposes of dynamic modeling the software package gPROMS (Version 3.0.4, Process System Enterprise Ltd., London, UK) was used. The kinetic model for the mass transfer between both phases contains Eqs. 3-5 to 3-7, where $c_{i, \text { org }}$ and $c_{i, a q}$ denote the concentrations of the compound $i$. $V_{\text {org }}$ and $V_{a q}$ stand for the volumes of the organic and aqueous phase, $A$ for the boundary area, and $\dot{n}_{i, M T}$ for the mass transfer flux.

$$
\begin{gathered}
\frac{d c_{i, \text { org }}}{d t} \cdot V_{\text {org }}=\dot{n}_{i, M T} \\
\frac{d c_{i, a q}}{d t} \cdot V_{a q}=-\dot{n}_{i, M T} \\
\dot{n}_{i, M T}=k_{i} \cdot A \cdot\left(P_{i} \cdot c_{i, a q}(t)-c_{i, \text { org }}(t)\right)
\end{gathered}
$$

According to the two-film theory (Lewis and Whitman, 1924) the mass transfer resistance in both boundary layers has to be considered. Therefore, the overall mass transfer coefficient $k_{i}$ is defined as follows:

$$
\frac{1}{k_{i}}=\frac{1}{k_{i, \text { org }}}+\frac{P_{i}}{k_{i, a q}}
$$

In Eq. 3-8 $k_{i, o r g}$ and $k_{i, a q}$ are the mass transfer coefficients within the organic and the aqueous boundary layer. However, this term contains only constants and thus can be combined to one single parameter in order to reduce the number of unknown parameters (Bauer et al., 2002; Mollerup and Hansen, 1998; Willeman et al., 2002a, 2002b). 


\subsection{Results and discussion}

\subsubsection{Ab initio solvent screening}

For the ab initio solvent screening using COSMO-RS 34 solvents have been tested for the non-reactive phase. Due to limitations of COSMO-RS the occurrence of $25 \%(\mathrm{v} / \mathrm{v}) \mathrm{DMF}$ was neglected for the ab initio solvent screening. The solvents have been chosen in a way that a wide range of solvent polarity is covered. Out of these, 11 solvents did not pass the first hurdle since they did not show a miscibility gap with water at $25^{\circ} \mathrm{C}$. For the remaining solvents, partition coefficients were calculated for the substrate DMBA and the product TMB between water and the organic phase. The solvents were assessed according to the partition coefficient of the product divided by the partition coefficient of the substrate. The obtained values for $R$ are listed in Table 3-1.

Table 3-1: $\quad$ List of solvents showing a miscibility gap in the COSMOtherm calculations with water at $25^{\circ} \mathrm{C}$, ranked according to their $R$ values.

\begin{tabular}{llll}
\hline Solvent & $R=P_{P} / P_{A}$ & Solvent & $R=P_{P} / P_{A}$ \\
\hline cis-decalin & 2.59 & benzene & 15.53 \\
cylcohexanone & 2.79 & 2-pentanone & 16.51 \\
n-heptane & 3.02 & piperidine & 16.69 \\
2-propanol & 3.28 & anisole & 16.84 \\
$n$-hexane & 3.35 & 1-butanol & 19.78 \\
cyclohexane & 3.56 & MTBE & 19.83 \\
pentane & 3.84 & acetophenone & 20.39 \\
1-propanol & 5.25 & 1-hexanol & 23.32 \\
tetrahydrofuran & 5.54 & MIBK & 24.14 \\
toluene & 12.41 & 1-pentanol & 24.15 \\
ethoxybenzene & 14.20 & diethylether & 24.42 \\
ethylacetate & 14.33 & & \\
\hline
\end{tabular}




\subsubsection{Parameter estimation}

To verify the COSMO-RS predictions two different solvents at extreme position in the solvent ranking were investigated experimentally. These two solvents were methyl-isobutylketone (MIBK) and $n$-hexane. For MIBK quite a large $R$ is predicted. Only diethylether exhibits a significant higher $R$ than MIBK. However, diethylether was disregarded due to its high volatility and safety issues. As example for a solvent with a relatively poor predicted $R$ $n$-hexane was chosen since extensive investigations have already been performed using this organic solvent for biphasic reaction systems (e.g. Ansorge-Schumacher, 2000; AnsorgeSchumacher et al., 2006; Hischer et al., 2005; Metrangolo-Ruiz de Temiño et al., 2005).

Due to minor deviations between the nominal and measured initial concentrations, the initial concentrations were individually corrected for each experiment prior to parameter estimation. Owing to the high correlation between the mass transfer coefficient and the partition coefficient the concurrent estimation of both parameters is difficult. Thus, in a first step only the mass transfer coefficient was estimated. For the partition coefficient the value obtained by HPLC was used (52.5 for DMBA, 89.8 for TMB). Afterwards, both parameters were estimated concurrently. The fit of the kinetic model to experimental data is shown in Figure 3-3 and Figure 3-4.

Good agreements between experiment and model can be observed for mass transfer experiments with DMBA, whereas those with TMB do not match as well. This problem may be caused by the lower purity and solubility of TMB compared to DMBA. This lower quality of the product mass transfer experiments affects the estimated model parameters (Table 3-2), which are less precise for TMB. However, the precision for all model parameters is satisfying. The correlation coefficients of the mass transfer coefficients and the partition coefficients is 1.0 for both DMBA and TMB. Despite this high correlation, the obtained parameter estimates can be considered to be reliable since the obtained values for the partition coefficients are very close to those obtained by HPLC. The obtained partition coefficients for DMBA and TMB result in a value of $1.611 \pm 0.023$ for $R$ using MIBK. 

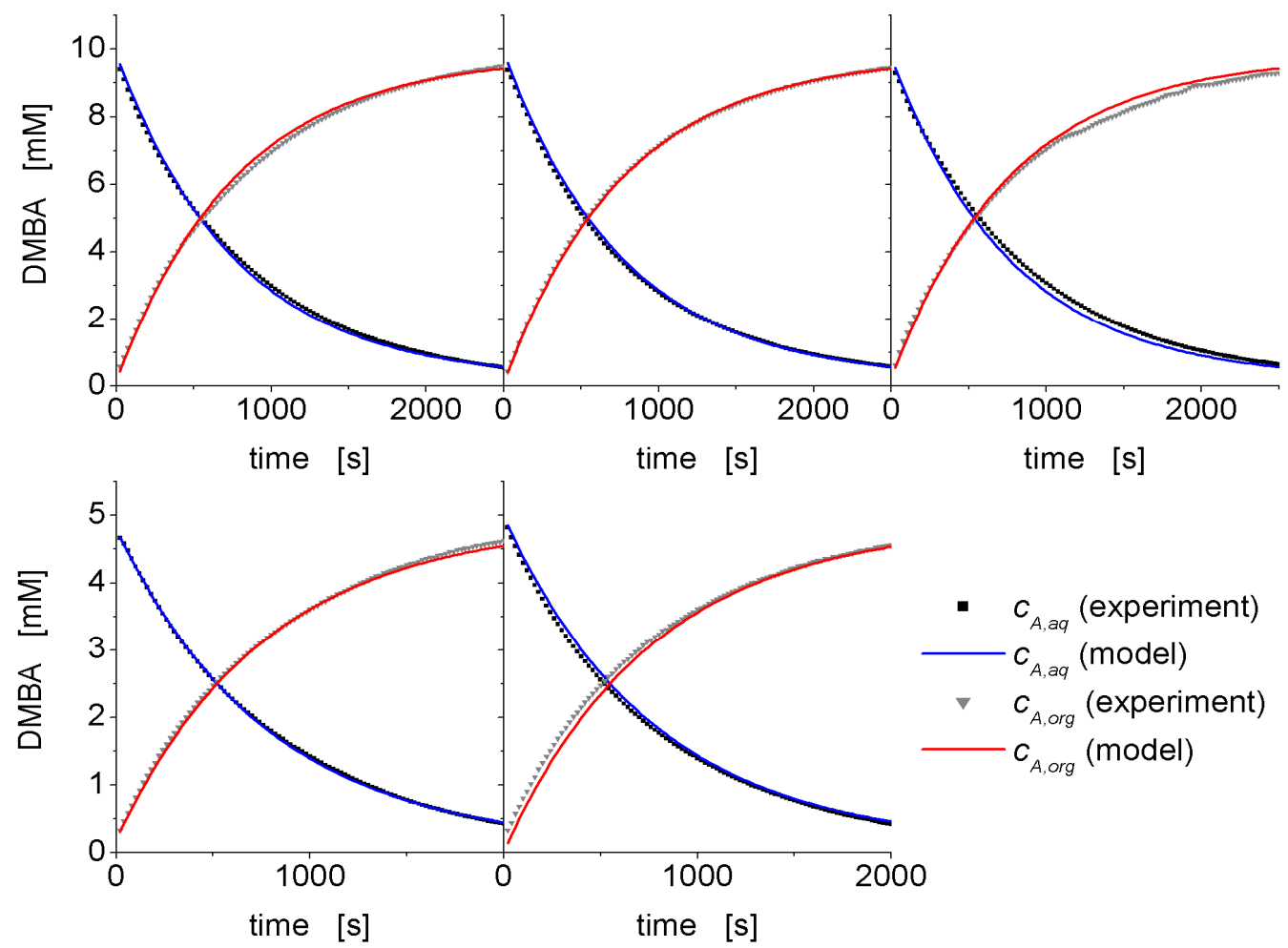

Figure 3-3: $\quad$ Fit of the kinetic model to five mass transfer experiments using the substrate DMBA and the organic solvent MIBK. The upper three experiments were started with the addition of $10 \mathrm{mM}$ DMBA to the aqueous phase, the lower two with $5 \mathrm{mM}$ DMBA $\left(25^{\circ} \mathrm{C}, 25 \%(\mathrm{v} / \mathrm{v}) \mathrm{DMF}\right)$.
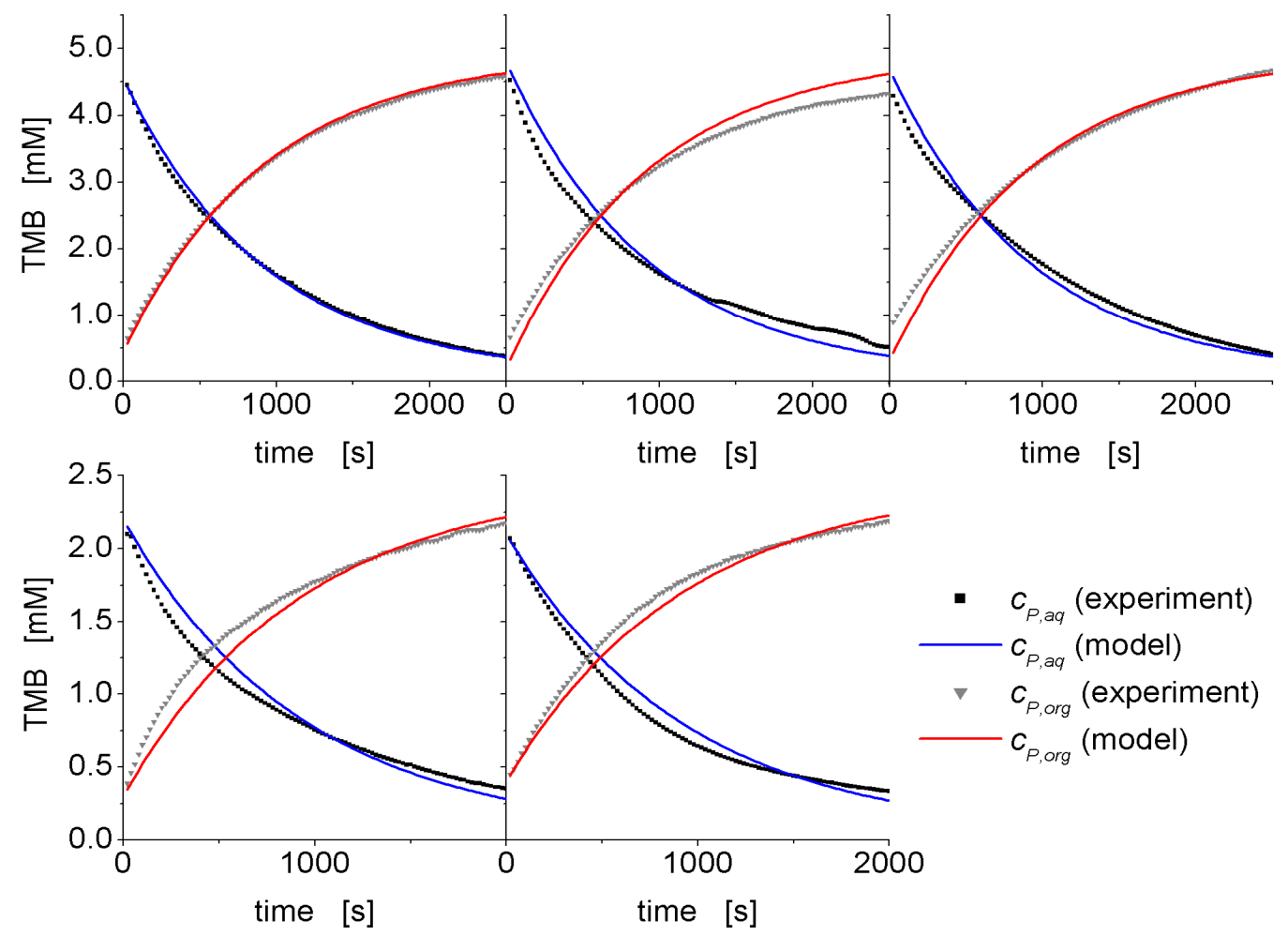

Figure 3-4: $\quad$ Fit of the kinetic model to five mass transfer experiments using the product TMB and the organic solvent MIBK. The upper three experiments were started with the addition of $5 \mathrm{mM}$ TMB to the aqueous phase, the lower two with $2.5 \mathrm{mM}$ TMB $\left(25^{\circ} \mathrm{C}, 25 \%(\mathrm{v} / \mathrm{v}) \mathrm{DMF}\right)$. 
Table 3-2: $\quad$ Estimated parameters for the kinetic mass transfer model $\left(25^{\circ} \mathrm{C}, 25 \%(\mathrm{v} / \mathrm{v}) \mathrm{DMF}\right)$.

\begin{tabular}{llll}
\hline model parameter & unit & estimate & standard deviation \\
\hline$k_{A}$ & $\mathrm{~m} \mathrm{~s}^{-1}$ & $5.39 \cdot 10^{-7}$ & $1.33 \cdot 10^{-9}$ \\
$k_{P}$ & $\mathrm{~m} \mathrm{~s}^{-1}$ & $2.81 \cdot 10^{-7}$ & $3.33 \cdot 10^{-9}$ \\
$P_{A}$ & - & 52.37 & 0.13 \\
$P_{P}$ & - & 84.36 & 0.99 \\
\hline
\end{tabular}

The same investigations were performed using $n$-hexane. Again, the kinetic model was fitted to experimental data (results not shown). The partition coefficients for DMBA and TMB using $n$-hexane are $3.41 \pm 0.04$ and $0.36 \pm 0.01$, respectively. Thus, a value of $0.106 \pm 0.004$ is obtained for $R$. This means that $n$-hexane is not suitable to obtain high yields. However, $R$ may be altered by process parameters such as $\mathrm{pH}$ value, ionic strength, temperature, and volume fraction of the cosolvent DMF. The investigation of the first two process parameters revealed that neither the $\mathrm{pH}$ value nor the ionic strength has a significant influence on $R$. This result seems reasonable since both reactants are uncharged. However, $R$ is affected by temperature. In Figure 3-5 it can be seen that the partition coefficients are changed only slightly by temperature, but in opposite directions. Therefore, $R$ can be strongly increased by temperature.

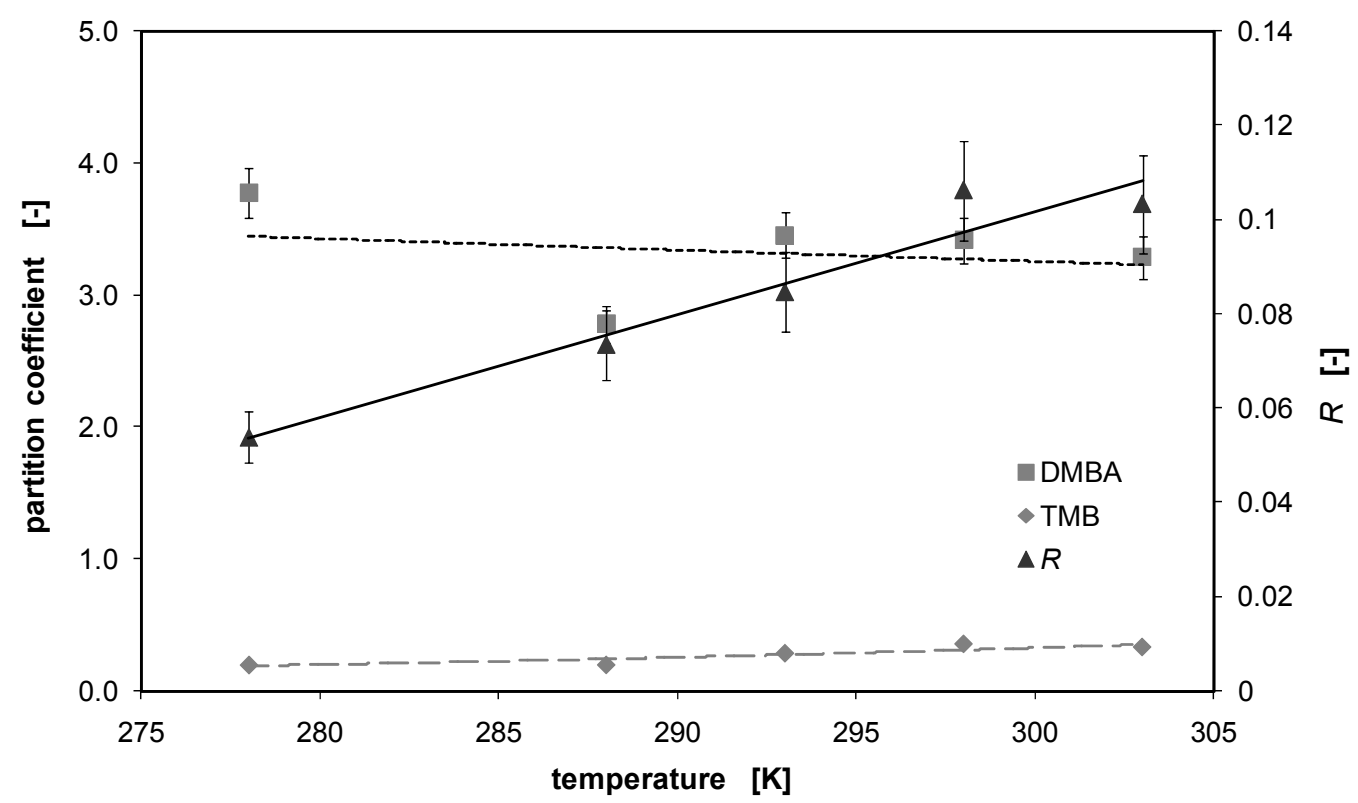

Figure 3-5: $\quad$ Influence of the temperature on the partition coefficients and on $R$ using $n$-hexane as organic solvent $(25 \%(\mathrm{v} / \mathrm{v}) \mathrm{DMF})$. The partition coefficients were determined after 24 hours using HPLC. 
More pronounced is the effect of the cosolvent DMF (Figure 3-6). DMF increases the equilibrium concentration of both reactants in the aqueous phase. However, the partition coefficient of the product is decreased more than the partition coefficient of the substrate. As a result, $R$ can be increased by adding less cosolvent to the aqueous phase. A process using $n$-hexane as extraction solvent should thus be operated at high temperatures with a minimal volume fraction of the cosolvent DMF.

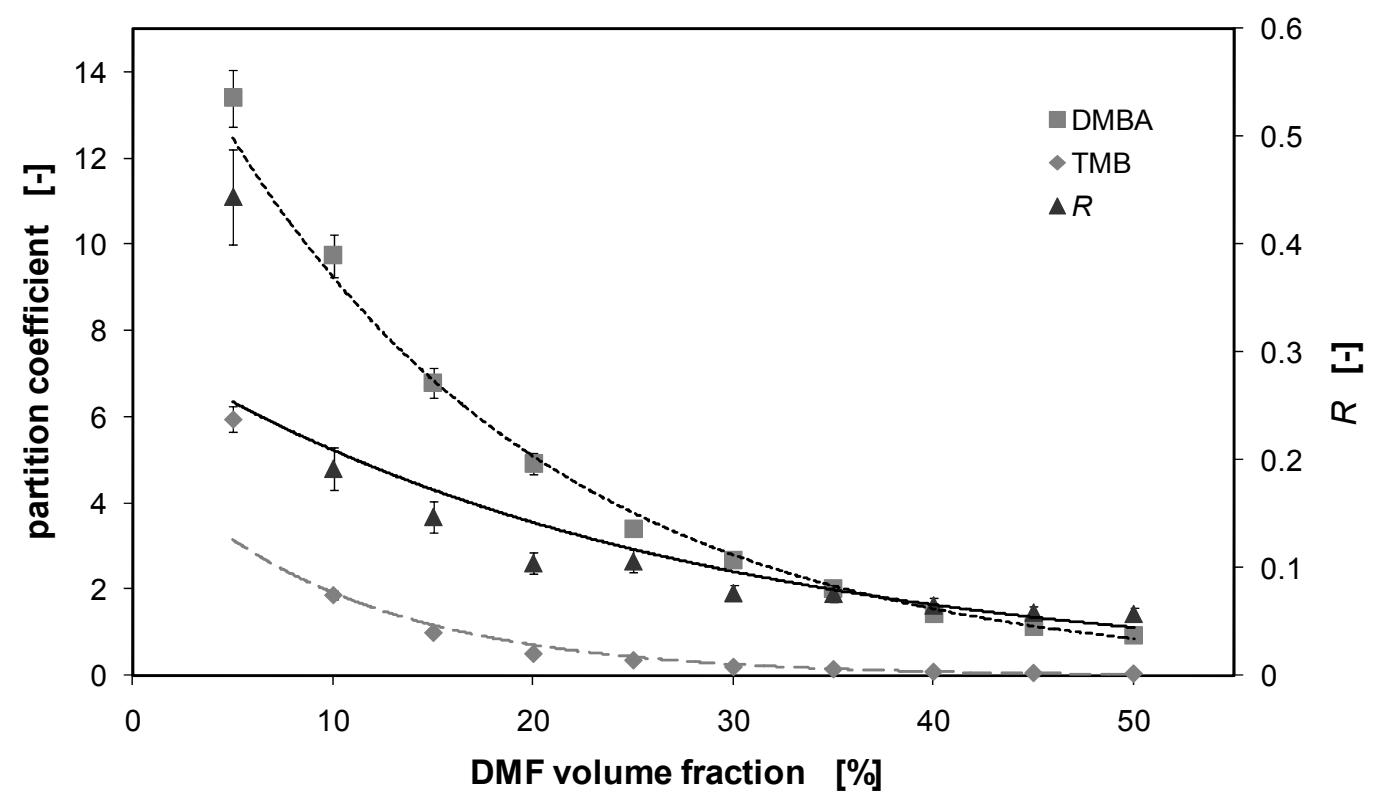

Figure 3-6: $\quad$ Influence of the volume fraction of the cosolvent DMF on partition coefficients and on $R$ using $n$-hexane as organic solvent $\left(25^{\circ} \mathrm{C}\right)$. The partition coefficients were determined after 24 hours using HPLC.

\subsubsection{Evaluation of $a b$ initio predictions}

The values of $R$, which were determined via fitting the kinetic model to experimental data, are significantly lower than those predicted by COSMO-RS (Table 3-1). One of the reasons is that in the COSMOtherm calculations the cosolvent DMF could not be considered. However, in Figure 3-7 it becomes obvious that despite the fact that their absolute values are not predicted precisely, the overall trend is verified, which is in line with previous investigations (Eckstein et al., 2006b; Spiess et al., 2008a). In future studies more solvents should be tested in order to investigate the reliability of the COSMO-RS predictions. 
COSMO-RS

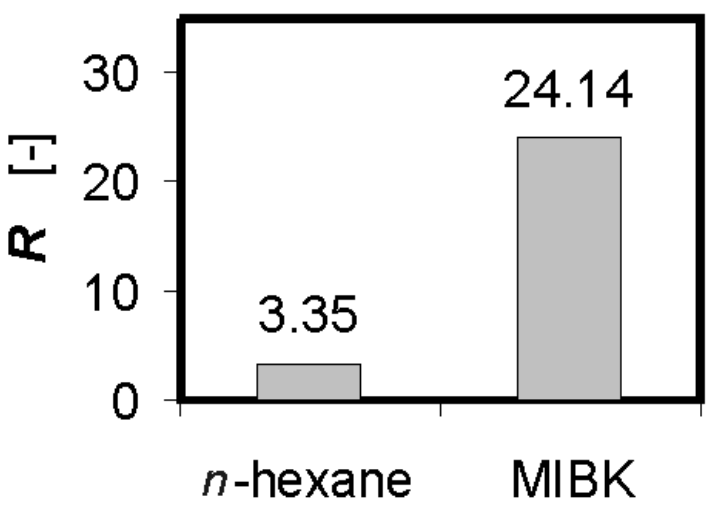

Experiment

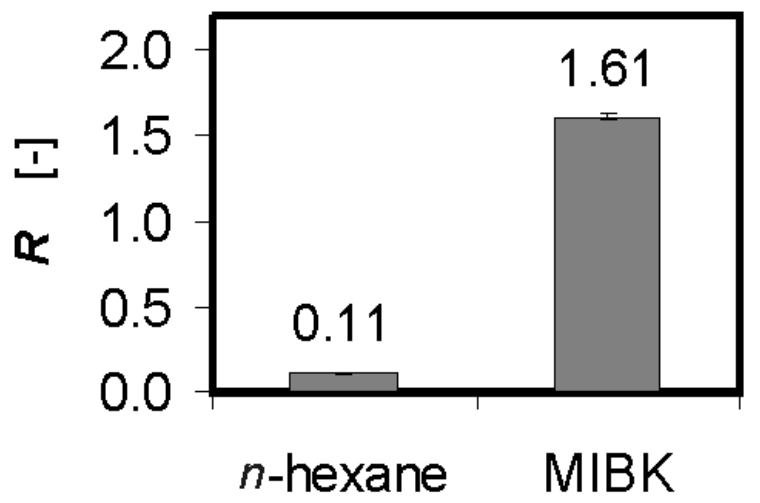

Figure 3-7: Comparison of ab initio predictions of COSMO-RS with the values obtained after fitting the kinetic model to experimental data.

As predicted by COSMO-RS MIBK exhibits a significantly higher $R$ than $n$-hexane. Consequently, MIBK is a more suitable solvent for the reaction in terms of the maximum extractable yield, which can be achieved. Certainly, there are many more parameters than $R$ that have an influence on the final solvent choice for an industrial process, such as volatility, safety issues, environmental aspects, price and availability (Fernandes and Cabral, 2008). Of special importance is the enzyme stability against molecular and phase toxicity, which can be caused by an organic solvent (Baldascini and Janssen, 2005; Butler, 1979; Halling, 1994; Villela Filho et al., 2003). Mostly, solvent selection in industry is a compromise.

\subsection{Conclusions}

In this chapter, a systematic approach to solvent selection for aqueous-organic biphasic reaction systems is presented. The aim of this approach is to identify a solvent for the nonreactive phase, which allows high extractable product yields for the carboligation of two DMBA molecules to TMB.

In the first step, COSMO-RS is used as a fast and effective tool for a solvent screening. Although no reliable absolute values can be predicted, at least as long as the cosolvent is neglected, the method is nevertheless a valuable tool for a first ab initio solvent screening. The second step, where a dynamic model is fitted to experimental data, leads to a more detailed and exact study of the model system. Furthermore, not only the mass transfer 
coefficients and the partition coefficients can be determined, but also the dependency on process parameters such as temperature and cosolvent volume fraction, which can significantly affect the mass transfer as demonstrated.

According to the obtained results, MIBK turns out to be a suitable solvent for the studied reaction and thus is chosen for the subsequent analysis of the BAL kinetics in the aqueousorganic biphasic reaction system. 


\section{Diffusion in Hydrogels}

Hydrogels are increasingly used for the immobilization of biocatalysts, especially for the use of enzymes in aqueous-organic biphasic systems. The immobilization of enzymes in hydrogels provides a stabilized aqueous environment for the enzymes while being suspended in an organic solvent. However, the productivity in such gel-stabilized biphasic systems may be limited by diffusion of the reactants (Berendsen et al., 2006; Goncalves et al., 2008; Guisan et al., 1994; van Roon et al., 2006). Hence, it is essential to study the influence of the hydrogel matrix on the transport kinetics. This provides valuable information not only for gelstabilized biphasic systems, but also for slow-release systems like drug release in the human body (e.g. Alexander, 2008). In the first Section of this chapter the diffusion of propionic acid into Ca-alginate hydrogel beads is investigated. Propionic acid is an example of a dissociating species. Subsequently, the diffusion of 3,5-dimethoxy-benzaldehyde (DMBA) and $(R)$ $3,3^{\prime}, 5,5^{\prime}$-tetramethoxy-benzoin (TMB) in $\kappa$-carrageenan hydrogel beads is studied. DMBA and TMB are the reactants for the investigated enzyme reaction using benzaldehyde lyase (BAL) (Chapter 2). In both cases confocal laser scanning microscopy (CLSM) is applied. Finally, nuclear magnetic resonance (NMR) is evaluated as alternative to CLSM and thus employed to analyze the diffusion of DMBA in $\kappa$-carrageenan hydrogel cylinders. The NMR results are compared to those of CLSM.

\subsection{Propionic acid diffusion in Ca-alginate hydrogel beads}

\subsubsection{Introduction}

The diffusion in hydrogels has been investigated for several years. Diffusion coefficients are mostly determined using Fick's law and measurements are conducted in the bulk phase. However, the deviation of published diffusion coefficients in hydrogels indicates that this approach might not be sufficiently sensitive (Willaert and Baron, 1996). Especially the diffusion of charged species might require considering additionally electrostatic interactions as described by the Nernst-Planck law. According to Heinemann (2003) the diffusion of charged species in hydrogels is far from being understood due to lack of suitable measurement techniques and appropriate dynamic models. In order to develop optimal 
measurement techniques and to derive mechanistic kinetic models, a structured analysis methodology is necessary.

Consequently, the objective of this work is to discriminate between possible diffusion laws for the diffusion of propionic acid into Ca-alginate hydrogels. For this purpose, a spatially distributed kinetic model is implemented and an adequate measurement technique chosen. Due to the complexity of this task the model-based experimental analysis (MEXA) approach is used to guide the work process (Figure 1-2).

To investigate the diffusion kinetics of propionic acid the MEXA work process starts with the development of a mechanistic diffusion model based on previous knowledge (Section 4.1.2). The diffusion model is employed to perform a sensitivity analysis (Section 4.1.4) in order to design an optimal experimental strategy and to choose a suitable measurement technique. A priori simulations are carried out to check for identifiability and discriminability of model variants (Section 0). The experimental methods are described in Section 4.1.5. Afterwards, the diffusion model is used to design optimal experiments for model discrimination (Section 4.1.6). The experimental results (Section 4.1.7) are then used for parameter estimation and model discrimination (Section 4.1.8.1). According to the iterative nature of the MEXA work process, the diffusion model is refined and a new model discrimination is performed (Section 4.1.8.2).

\subsubsection{Model development}

The system of interest is a hydrogel bead suspended in an organic solvent that serves as supply of the substrate, which diffuses from the bulk phase into the bead. The hydrogel matrix consists of $\mathrm{Ca}$-alginate, which results from the gelation of alginate with $\mathrm{CaCl}_{2}$, releasing free chloride $\left(\mathrm{Cl}^{-}\right)$ions during cross-linking. The Ca-alginate matrix is considered immobile, but is slightly buffered using Tris-buffer to control the $\mathrm{pH}$ value during gelation. The chloride ions, the excess calcium ions, as well as Tris and $\mathrm{TrisH}^{+}$are supposed to be mobile. If an organic acid is used as substrate, the acid may dissociate to the corresponding basic anion. Fickian and Nernst-Planck diffusion laws may differ for the diffusion of charged compounds. Thus, the diffusion of propionic acid $(\mathrm{HPa})$ and its propionate anion $\left(\mathrm{Pa}^{-}\right)$in the hydrogel matrix is investigated. 
Assuming an ideally mixed batch system without volume change and ideally spherical particles, the mass balance equations for the bulk (Eq. 4-1) and the bead (Eq. 4-2) are derived, where $i$ denotes the diffusing species, and $j_{\mathrm{i}}$ the molar diffusive flux (in mol $\mathrm{m}^{-2} \mathrm{~s}^{-1}$ ). $V_{b u l k}$ represents the volume of the bulk phase, $k_{i}$ the mass transfer coefficients, $A_{\text {bead }}$ the surface of the bead, $R$ the bead radius, $r$ the radial position in the bead, $t$ the time, and $c_{i}$ the concentrations in the bead and the bulk phase, respectively.

$$
\begin{gathered}
V_{\text {bulk }} \frac{d c_{i, \text { bulk }}}{d t}=k_{i} \cdot A_{\text {bead }} \cdot\left(c_{i, \text { bead }}(r=R)-c_{i, \text { bulk }}\right) \\
\frac{\partial c_{i, \text { bead }}}{\partial t}=-\frac{1}{r^{2}}\left(\frac{\partial}{\partial r} \cdot r^{2} \cdot j_{i}\right)
\end{gathered}
$$

The boundary and initial conditions are shown in Eqs. 4-3 to 4-6:

$$
\begin{gathered}
j_{i}(r=0)=0 \\
j_{i}(r=R)=k_{i} \cdot\left(c_{i, \text { bead }}(r=R)-c_{i, \text { bulk }}\right), \quad i=H P a \\
c_{i, \text { bulk }}(t=0)=c_{i, \text { bulk }, 0} \\
c_{i, \text { bead }}(t=0)=c_{i, \text { bead }, 0}
\end{gathered}
$$

The diffusion rate of charged species at constant pressure may be described using NernstPlanck law (Taylor and Krishna, 1993), where the area-specific flux of a charged component is driven by a combination of the gradients in concentration and electrical potential:

$$
j_{i}=-D_{i} \cdot\left(\frac{\partial c_{i}}{\partial r}+z_{i} \cdot c_{i} \cdot \frac{F}{R_{m} \cdot T} \cdot \frac{\partial \Phi}{\partial r}\right)
$$

In Eq. 4-7 $D_{i}$ denotes the effective diffusion coefficient, which takes the influence of the alginate matrix into account, $z_{i}$ the charge, $F$ the Faraday constant, $R_{m}$ the molecular gas constant, $T$ the temperature, and $\Phi$ the electrical potential. The gradient in potential can be expressed in terms of concentrations by summing up the concentration gradients of all involved species. This yields a representation of Nernst-Planck law (Eq. 4-8) based merely on 
ion concentrations, ion diffusivities and ion charges (Taylor and Krishna, 1993; van Roon et al., 2006):

$$
j_{i}=-D_{i} \cdot \frac{\partial c_{i}}{\partial r}-\frac{z_{i} \cdot D_{i} \cdot c_{i}}{\sum_{h} z_{h}^{2} \cdot D_{h} \cdot c_{h}} \cdot \sum_{h} z_{h} \cdot D_{h} \cdot \frac{\partial c_{h}}{\partial r}
$$

For uncharged species $\left(z_{i}=0\right)$, the second summand equals zero, resulting in Fick's law (Eq. 4-9):

$$
j_{i}=-D_{i} \cdot \frac{\partial c_{i}}{\partial r}
$$

As already stated, the diffusing species are electrolytes that may dissociate depending on the environment. The dissociation process is fast in comparison to the diffusion. Therefore, it is assumed that the dissociation reaction is in equilibrium at all times. The dissociation equilibrium is represented by the mass action laws (Eqs. 4-10 and 4-11), where $K_{i}$ is the dissociation constant, the $c_{i}^{\prime}$ 's are the concentrations of the undissociated electrolytes, the cation, and the anion, respectively, while $K_{w}$ is the ion product of water, $c_{H^{+}}$the proton and

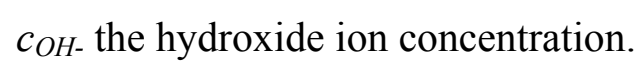

$$
\begin{gathered}
K_{i}=\frac{C_{i, \text { cation }} \cdot C_{i, \text { anion }}}{C_{i, \text { electrolyte }}} \\
K_{w}=C_{H^{+}} \cdot C_{\mathrm{OH}^{-}}
\end{gathered}
$$

Since electroneutrality is given at all times, expressed by the charge balance (Eq. 4-12), one diffusive flux can be expressed through the diffusion of all other species.

$$
\sum_{i} c_{i} \cdot z_{i}=0
$$

The $\mathrm{pH}$ value is calculated using Eq. 4-13:

$$
p H=-\log c_{H^{+}}
$$


The diffusion coefficient $D_{i}$ in the hydrogel bead matrix may depend on the alginate density. The Ca-alginate beads are not homogeneous, but the alginate polymer is radially distributed. During the gelling process, the bivalent Ca-ions diffuse from the surface into the bead and rapidly cross-link the alginate, resulting in a higher concentration near the surface than in the core (Skjak-Braek et al., 1989; Thu et al., 2000). For the bead production process used in this work, the alginate density distribution has been measured using Raman spectroscopy (Heinemann et al., 2005). The quadratic equation 4-14 was used to fit the alginate density profile, where $w_{\text {alg }}$ is the alginate mass fraction, $\bar{w}_{\text {alg }}$ the average alginate mass fraction, $d$ a model parameter to be fitted, and $R$ the bead radius. The quadratic dependence is in accordance with previous observations (Martinsen et al., 1992; Skjak-Braek et al., 1989).

$$
w_{\text {alg }}(r)=\bar{w}_{\text {alg }}+d \cdot\left(r^{2}-\frac{3}{5} \cdot R^{2}\right)
$$

Masaro and Zhu (1999) list several physical models mainly for self-diffusion of small to medium size non-electrolytes in polymer solutions in their review article. The presented model alternatives express the diffusion coefficient $D_{i}$ as a function of the diffusion coefficient in free solution $D_{i, 0}$ and a tuneable model parameter $k$ that indicates the extent of the matrix interaction. The most simplified models take exponential (hydrodynamic theory, model of Gao and Fagerness (1995), Eq. 4-15), hyperbolic (simplified Maxwell-Fricke model, Eq. 4-16), or linear form (obstruction model for micro-emulsions, Eq. 4-17), and are expressed in terms of the alginate mass fraction.

$$
\begin{gathered}
D_{i}=D_{i, 0} \cdot e^{-k \cdot w_{a l g}} \\
D_{i}=\frac{D_{i, 0}}{1+k \cdot w_{a l g}} \\
D_{i}=D_{i, 0} \cdot\left(1-k \cdot w_{a l g}\right)
\end{gathered}
$$

In order to perform the model analysis, nominal values have to be assigned to all model parameters. An overview of the model parameters and the assigned values is provided in Table 4-1. Assuming a $\mathrm{pH}$ range between 3 and 8 during the diffusion process, the very strong acid $\mathrm{HCl}$ is fully dissociated. Also most salt species are fully dissociated. To reduce the computational load, species that only occur theoretically are omitted in the model. 
Table 4-1: $\quad$ Model parameters describing the diffusion of propionic acid into Ca-alginate hydrogel beads (Heinemann, 2003).

\begin{tabular}{ll}
\hline Species & Diffusion coefficient in free solution $D_{i, 0}\left(\mathrm{~m}^{2} \mathrm{~s}^{-1}\right)$ \\
\hline $\mathrm{HPa}$ & $1.061 \cdot 10^{-9}$ \\
$\mathrm{~Pa}$ & $0.953 \cdot 10^{-9}$ \\
$\mathrm{TrisH}^{+}$ & $0.778 \cdot 10^{-9}$ \\
$\mathrm{Tris}$ & $0.700 \cdot 10^{-9}$ \\
$\mathrm{CaCl}^{+}$ & $1.000 \cdot 10^{-9}$ \\
$\mathrm{Ca}^{2+}$ & $0.792 \cdot 10^{-9}$ \\
$\mathrm{Cl}^{-}$ & $2.032 \cdot 10^{-9}$ \\
$\mathrm{OH}^{-}$ & $5.273 \cdot 10^{-9}$ \\
$\mathrm{H}^{+}$ & $9.312 \cdot 10^{-9}$ \\
\hline $\mathrm{Dissociation}^{+}$pair & Dissociation constants, $\mathrm{p} K_{i}=-\log \mathrm{K}_{i}$ \\
\hline $\mathrm{HPa}^{-} \mathrm{Pa}^{-}$ & 4.860 \\
$\mathrm{TrisH}^{+} / \mathrm{Tris}^{2}$ & 8.000 \\
$\mathrm{CaCl}^{+} / \mathrm{Ca}^{2+}$ & -0.140 \\
\hline $\mathrm{Dissociation}$ pair & lon product of water, $\mathrm{p} K_{w}=-\log K_{w}$ \\
\hline $\mathrm{H}_{2} \mathrm{O} / \mathrm{OH}^{-}$ & 13.996 \\
\hline
\end{tabular}

\subsubsection{A priori simulations to check for discriminability}

The discriminability of the diffusion law variants (different assumptions on the dependence of the effective diffusion coefficient on the alginate density, Eqs. 4-15-17, hereafter referred to as alternative diffusion coefficient models) is investigated using a priori simulations of the $\mathrm{pH}$ profiles under identical initial conditions. The calculated influence of the alternative diffusion coefficient models on the resulting $\mathrm{pH}$ profiles at different times is shown in Figure 4-1. 


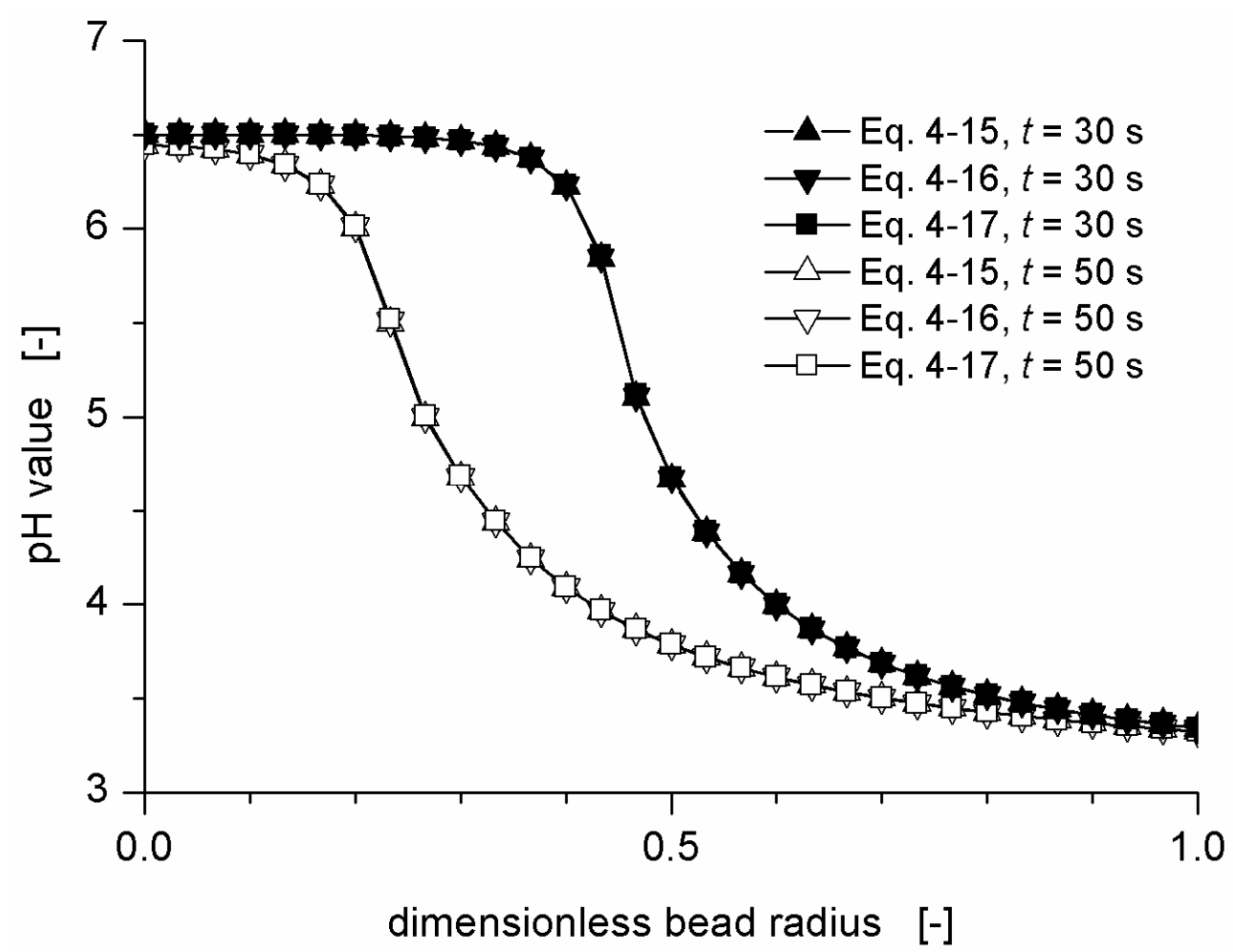

Figure 4-1: $\quad$ Simulated $\mathrm{pH}$ value over time in the hydrogel particle core after $30 \mathrm{~s}$ and $50 \mathrm{~s}$ assuming different (linear, exponential, hyperbolic) dependence of the diffusion coefficient on the local alginate density (Eqs. 4-15-17). Nernst-Planck diffusion is chosen as diffusion model. Simulation parameters: $R=1.15 \mathrm{~mm}, \mathrm{p} H_{\text {bead }, 0}=6.5, c_{P a, t o t, 0}=100 \mathrm{mM}, \mathrm{w}_{\mathrm{alg}}=3.3 \%(\mathrm{w} / \mathrm{w})$, $d=6000 \mathrm{~g} \mathrm{~g}^{-1} \mathrm{~m}^{-2}, k=6.60 \mathrm{~g} \mathrm{~g}^{-1}$.

These simulations show no visible difference in the $\mathrm{pH}$ profiles calculated for the alternative diffusion coefficient models (Eqs. 4-15-17). Inspecting the numerical data, the largest simulated difference occurs at the center of the bead where it amounts to less than $0.0066 \mathrm{pH}$ units. Even using highly precise $\mathrm{pH}$ measurement methods, these differences are an order of magnitude smaller than the typical measurement errors. Therefore, the a priori simulations show that a discrimination of the alternative diffusion model variants for low molecular weight substrates cannot be expected using hydrogels of this density and size. In the following Sections, the exponential dependence of the diffusion coefficient on alginate density (Eq. 4-15) was chosen to account for the radial variation of the diffusion coefficient and the task of model discrimination was re-focused to discrimination between Fick and Nernst-Planck diffusion laws. 


\subsubsection{Sensitivity analysis}

Prior to model discrimination the matrix interaction parameter $k$ has to be experimentally determined. For this purpose, two experimental strategies are possible. In the bulk the propionic acid concentration can be assessed directly by e.g. taking samples. In contrast to this, non-invasive spectroscopic measurements are required to get information from the inside of the bead. This could be achieved by using a $\mathrm{pH}$ indicator and measuring the $\mathrm{pH}$ value. Thus, the direct measurement of the propionic acid concentration in the bulk phase and the indirect $\mathrm{pH}$ measurement in the bead center are compared. In order to decide for a suitable experimental strategy, a sensitivity analysis is carried out using the developed diffusion model.

For the simulations the following realistic parameter values are used: The nominal bead radius $R$ is set to $1.15 \mathrm{~mm}$. The hydrogel beads contain ${ }^{-} \bar{w}_{\text {alg }}=3.3 \%(\mathrm{w} / \mathrm{w})$ sodium-alginate in $50 \mathrm{mM}$ Tris-buffer and have been hardened with $\mathrm{CaCl}_{2}$ to yield a bead concentration of $180 \mathrm{mM} \mathrm{CaCl}_{2}$. The alginate distribution has been fitted to measured alginate concentration profiles (Heinemann et al., 2005), giving an alginate parameter $d=6000 \mathrm{~g} \mathrm{~g}^{-1} \mathrm{~m}^{-2}$ (Eq. 4-14). The initial $\mathrm{pH}$ value $p H_{0}$ is set to 6.5 and the volume of the bulk solution to $V_{b u l k}=300 \mu \mathrm{L}$. The initial total concentration of propionic acid $c_{P a, t o t, 0}$ is set to $100 \mathrm{mM}$. For the sensitivity analysis, the Nernst-Planck law and the exponential diffusion law variant of Gao and Fagerness (1995) (Eq. 4-15) are employed. Figure 4-2 depicts the normalized sensitivities of the matrix interaction parameter $k$, the bead radius $R$, and the bulk volume $V_{\text {bulk }}$. These were obtained by using Eqs. 4-18 and 4-19 and approximated with finite differences. $\theta_{i}$ denotes the respective parameters.

$$
\begin{gathered}
S_{i, H P a, b u l k}=\frac{\theta_{i}}{C_{H P a, b u l k}} \cdot \frac{\partial c_{H P a, b u l k}}{\partial \theta_{i}} \\
S_{i, p H(r=0)}=\frac{\theta_{i}}{p H(r=0)} \cdot \frac{\partial p H(r=0)}{\partial \theta_{i}}
\end{gathered}
$$

Figure 4-2 clearly shows that the values of the normalized sensitivity for the matrix interaction parameter are much higher, if measurements in the bead center are conducted in comparison to bulk measurements. Moreover, the influence of the matrix interaction parameter on the concentration in the bulk phase is superimposed by the influences of both 
the bead radius and the bulk volume. Thus, minor pipetting errors during bulk phase addition can cause major errors in the estimated value for the matrix interaction parameter. On the contrary, the estimate for the matrix interaction parameter is virtually independent of errors in the bulk volume, if $\mathrm{pH}$ measurements in the bead center are conducted. However, for both experimental strategies, the estimate is superimposed by the bead radius. Therefore, the bead radius should be accurately measured.
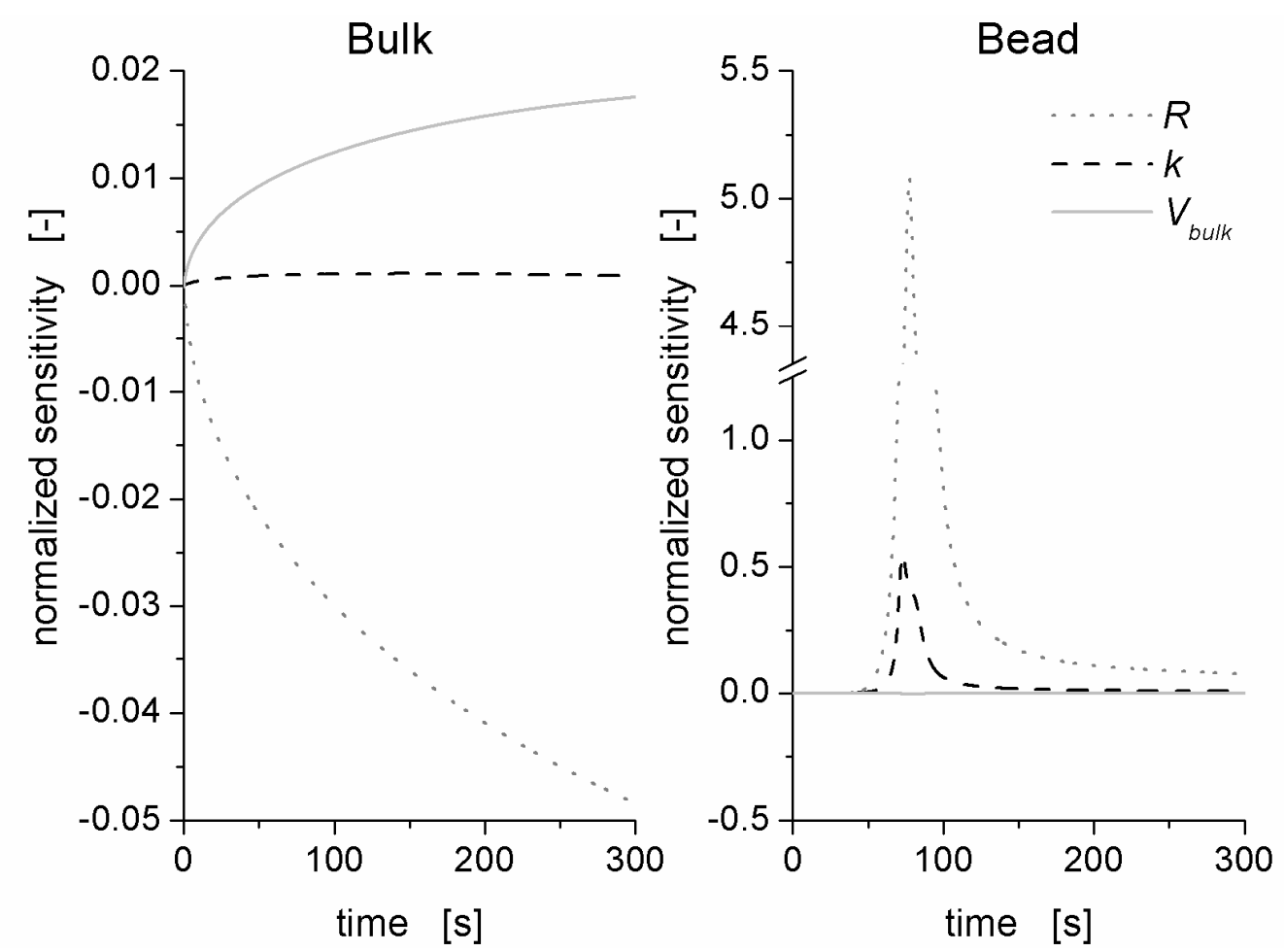

Figure 4-2: $\quad$ Sensitivity analysis for the parameters bead radius $R$, matrix interaction parameter $k$, and bulk volume $V_{b u l k}$ in the bulk phase (left) and in the bead center (right). For bulk measurements the sensitivity refers to the propionic acid concentration, for the bead measurements to the $\mathrm{pH}$ value $\left(R=1.15 \mathrm{~mm}, \mathrm{p} H_{\text {bead }, 0}=6.5, c_{P a, t o t, 0}=100 \mathrm{mM}, V_{\text {bulk }}=300 \mu \mathrm{L}, k=6.60 \mathrm{~g} \mathrm{~g}^{-1}\right)$.

On the basis of these results, it can be concluded that the traditional experimental strategy, measurements in the bulk phase (e.g. Polakovic et al., 2001), provides only integral information and thus is not suitable for the determination of diffusion-related parameters in polymer beads. For this reason, measurements inside the beads are required.

This can be achieved with spectroscopic measurement techniques, which are increasingly applied for the non-invasive in situ observation of chemical species. Nuclear magnetic resonance (NMR) techniques have recently been applied for the imaging of transport phenomena and chemical reactions (Küppers et al., 2002). In principle, any chemical species 
can be observed using NMR imaging. However, a trade-off between spatial, chemical and temporal resolution has to be made. Alternatively, Fourier transformed infrared (FTIR) spectroscopy (Frazier et al., 2001) or Raman spectroscopy have also been used for the spatially resolved observation of concentration and for the determination of diffusion coefficients in hydrogel slabs (Kwak and Lafleur, 2003). The applicability of Raman spectroscopy, however, is limited because only non-fluorescent species of significant concentration can be quantified so far. In contrast, the widely applied confocal laser scanning microscopy (CLSM) can only observe fluorescent species. Therefore, CLSM requires either fluorescent molecules (Tallarek et al., 2003, Cvetkovic et al., 2004), or small fluorescent marker molecules attached to macromolecules to allow for spatially resolved concentration measurements (Kasche et al., 2003). The quantification of concentration based on fluorescence intensity, however, has been demonstrated to be limited by the attenuation of light by the carrier matrix that results in depth-dependent calibration curves and prohibits the evaluation of (fast) dynamic diffusion problems (Heinemann et al., 2004). The impact of light attenuation may be overcome using either two photon excitation (Denk et al., 1990), or fluorescence lifetime CLSM (Kuwana et al., 2004). Due to its excellent temporal and spatial resolution fluorescence lifetime CLSM is chosen from the available spectroscopic techniques for the $\mathrm{pH}$ measurements in the bead center.

\subsubsection{Experimental methods}

Propionic acid, Tris base, and calcium chloride were purchased from Fluka (Buchs, Switzerland). Resorufin was obtained from Sigma-Aldrich (Deisenhofen, Germany). Manugel DJX sodium alginate was purchased from Monsanto (San Diego, CA, USA). All chemicals used were of analytical grade.

For the preparation of the hydrogel beads, first, $2 \%(\mathrm{w} / \mathrm{v})$ sodium alginate were dissolved in $50 \mathrm{mM}$ Tris buffer, $\mathrm{pH}$ 6.8. To obtain almost ideally round beads, $15 \mu \mathrm{L}$ of alginate solution were dropped using a pipette into a column containing a $\mathrm{Ca}^{2+}$ and a density gradient (Buthe et al., 2004). Due to the minimization of the interface round drops are formed. During sedimentation $\mathrm{Ca}^{2+}$ ions diffuse into the drops and cross-link the alginate. Finally, hydrogel beads of approximately $1 \mathrm{~mm}$ radius were obtained. The beads were washed several times with $50 \mathrm{mM}$ Tris buffer containing $2 \% \mathrm{CaCl}_{2}$ and $500 \mu \mathrm{M}$ resorufin as fluorescent lifetime $\mathrm{pH}$ indicator. 
The diffusion experiments were performed in a 96-well microtiter plate at room temperature. To guarantee uniform conditions around the hydrogel bead, it was placed on top of a circular holder supporting the bead at three points above the well bottom. The diffusion experiment was initiated adding $300 \mu \mathrm{L}$ of propionic acid solution $c_{H P a, t o t, 0} \sim 100 \mathrm{mM}$ to the well. Solvent evaporation was considered negligible due to the short duration of the experiment.

lateral view

camera
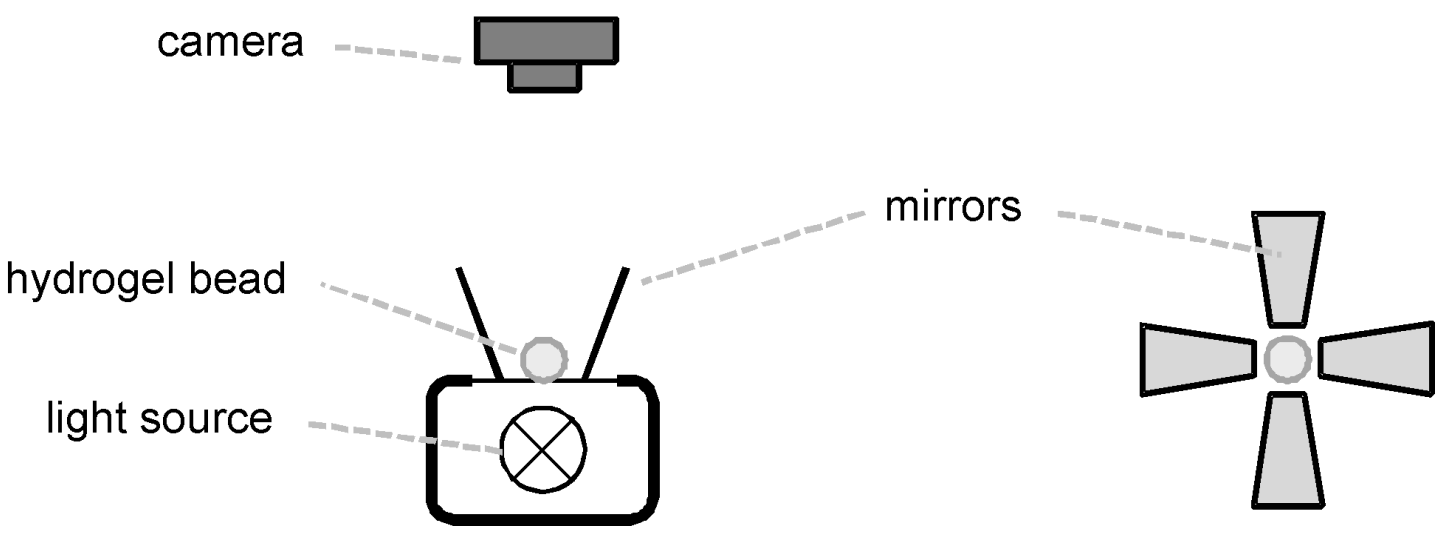

Figure 4-3: Device for the determination of bead roundness. Digital pictures were taken from an illuminated hydrogel bead and its four mirror images and subsequently analyzed quantitatively.

After each measurement, the position and the quality of the hydrogel bead was controlled using light microscopy. Data from beads that had not been correctly centered or that contained cracks or air inclusions were rejected. Since the sensitivity analysis revealed that an exact bead radius has to be known to estimate the matrix interaction parameter precisely, the bead radius and the roundness were subsequently measured using the device depicted in Figure 4-3 and the image analysis software Image Tool 3.00 (UHTSCSA, San Antonio, TX, USA). Beads with a roundness $\left(=R_{\min } / R_{\max }\right)$ less than 0.8 were rejected likewise.

For the lifetime measurements, a Leica TCS SP2 confocal laser scanning microscope (Leica Microsystems Heidelberg GmbH, Mannheim, Germany) was combined with a pulsed $405 \mathrm{~nm}$ diode (PicoQuant, Berlin, Germany) emitting picosecond laser pulses for the excitation of resorufin fluorescence. Only fluorescence photons emitted from the confocal plane can pass a confocal pinhole and are collected through a longpass filter (edge $500 \mathrm{~nm}$ ) by a photomultiplier tube (PMT) with high quantum efficiency and accurate timing characteristics 
(H7422P-40, Hamamatsu Photonics GmbH, Herrsching, Germany). The electrical pulses from the PMT are guided to an external PC system for photon counting (SPC 830, Becker and Hickl, Berlin, Germany), that correlates the counted photons to the excitation laser pulses via time-correlated single photon counting. During all experiments, the average power of the laser diode was kept at $50 \mu \mathrm{W}$, and the repetition rate at $20 \mathrm{MHz}$. The diffusion process was observed for $300 \mathrm{~s}$, divided into 300 observation cycles of $1 \mathrm{~s}$ each to balance time resolution with sufficient number of photons. During this second all recorded photons were accumulated to calculate one lifetime decay curve.

For the calibration of the correlation between the $\mathrm{pH}$ value and the resorufin fluorescence lifetime, resorufin-doted sodium alginate solutions and Ca-alginate hydrogel beads were equilibrated at different $\mathrm{pH}$ values. In each of those samples, the decay of resorufin fluorescence intensity after the excitation pulses was recorded tenfold using all wavelengths larger than $500 \mathrm{~nm}$. The acidic and basic forms of resorufin have different $\mathrm{pH}$ independent lifetimes (Ryder et al., 2003). At a defined $\mathrm{pH}$ value, the decay curve is composed of lifetime components of both forms. Thus, a biexponential intensity decay over time is obtained, which can be described with Eq. 4-20.

$$
I=a \cdot e^{-\frac{t}{\tau_{A}}}+b \cdot e^{-\frac{t}{\tau_{B}}}
$$

In Eq. 4-20 $I$ denotes the normalized time-dependent intensity and $t$ the time. The preexponential factors $a$ and $b$ are the proportions of the acidic and basic component that sum up to 1 , and $\tau_{A}$ and $\tau_{B}$ are the corresponding lifetimes. The obtained lifetime curves of resorufin are fitted using Eq. 4-20 with fixed lifetimes in order to obtain the proportions $a$ and $b$ at different $\mathrm{pH}$ values. The resulting proportion of acidic to basic component over $\mathrm{pH}$ value in the hydrogel bead is depicted in Figure 4-4. 


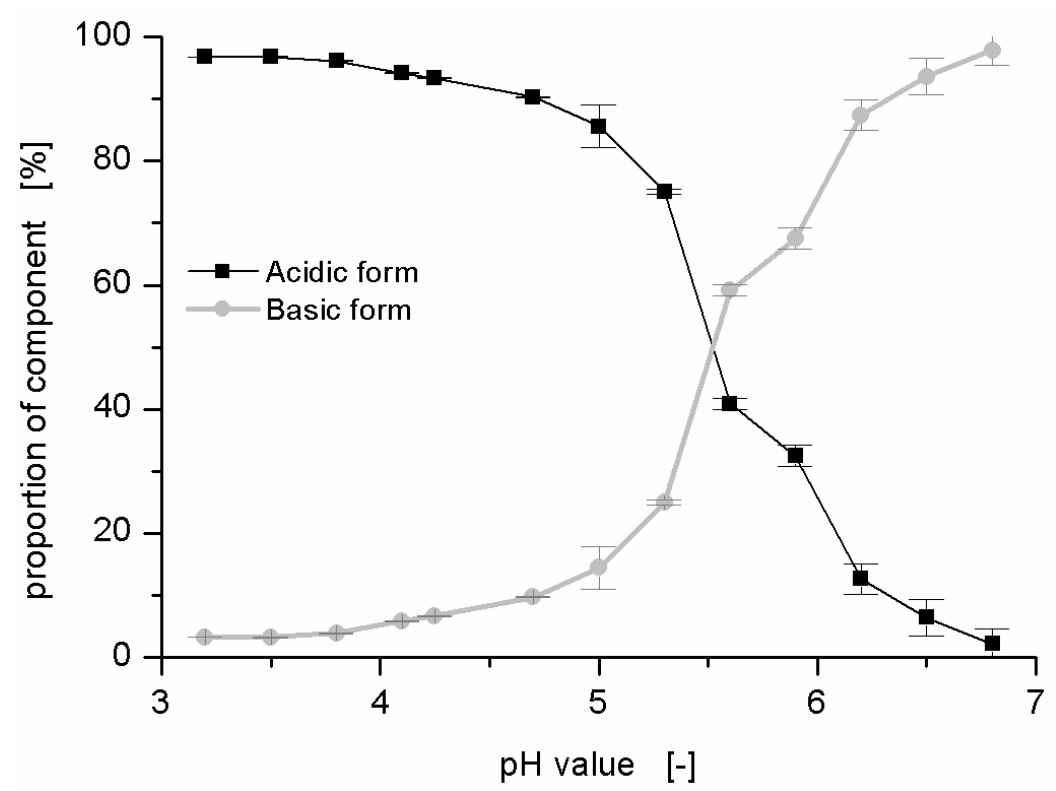

Figure 4-4: $\quad$ Calibration of the $\mathrm{pH}$ value vs. the proportion of acidic and basic dissociation forms $a$ and $b$ of resorufin as determined from biexponential decay curves. The error bars indicate the standard deviation based on 10 repetitions.

Figure 4-4 shows the typical shape of a $\mathrm{pH}$ titration curve with a $\mathrm{pK}$ value of approximately 5.6 , where nearly pure acidic or basic form of the $\mathrm{pH}$ indicator resorufin is observed at roughly $1.5 \mathrm{pH}$ units below and above the $\mathrm{pK}$ value. The lifetimes of the acidic and basic component of $0.4 \mathrm{~ns}$ and $2.6 \mathrm{~ns}$, respectively, coincide satisfactorily with the data of $0.3-$ 0.67 ns and 3.3-3.4 ns reported earlier (Ryder et al., 2003). The deviations may be due to the chosen excitation and observation wavelength ranges and the assumption of fixed component lifetimes across all $\mathrm{pH}$ values. The standard deviation of the replicates is between $0.3 \%$ and $3 \%$, with the higher error occurring at high $\mathrm{pH}$ values. The increase of experimental error with increasing $\mathrm{pH}$ value is due to the different intensity of both dissociating forms of resorufin. At basic $\mathrm{pH}$ values, the anion with a lower intensity prevails, resulting in the lifetime decay curve consisting of fewer counted photons. In independent experiments, it has been verified that the scanning depth in the hydrogel bead does not influence the lifetime measurement (data not shown). The calibration curve for the acidic component $a$ (Figure 4-4) decreases monotonously with increasing $\mathrm{pH}$ value, however, the curve shows a slight shoulder around $\mathrm{pH}$ 5.5. For the evaluation of the diffusion experiments, a linear interpolation between the calibration points is applied to estimate the $\mathrm{pH}$ value. 


\subsubsection{Optimal experimental design for model discrimination}

Based on the experimental setup, optimal experiments for discrimination between Fickian and Nernst-Planck diffusion can be designed. According to the simple approach of Hunter and Reiner (1965), an optimal experiment is defined as the one giving the largest difference between both competing model candidates. Numerically, this refers to the maximization of the objective function $\Psi(\varphi)$ with respect to the experimental degrees of freedom $\varphi$ (Eq. 4-21). $\theta^{N}$ and $\theta^{F}$ are the parameter sets for Fickian and Nernst-Planck diffusion, respectively, $t_{\text {exp }}$ denotes the experiment duration.

$$
\max _{\varphi} \Psi(\varphi)=\max _{\varphi} \int_{t=0}^{t=t_{\text {exp }}} \sum_{i}\left[p H_{i}^{N}\left(r, t, \boldsymbol{\theta}^{N}, \varphi\right)-p H_{i}^{F}\left(r, t, \boldsymbol{\theta}^{F}, \varphi\right)\right]^{2} d t
$$

The objective function integrates the predictions of $\mathrm{pH}$ differences over time in order to determine the experimental conditions at which the model alternatives can be discriminated most efficiently. The experimental degrees of freedom $\varphi$ for the optimization are the measurement position $r$ within the hydrogel bead, the hydrogel bead size, the initial $\mathrm{pH}$ value of the bead, and the initial concentration of the propionic acid in the bulk.

Regarding the optimal measurement position $r$ during an experiment, different scanning strategies can be considered. The most simple approach is to measure at a single, spatial position for the complete course of the reaction. Alternatively a scan along a line or across a plane is possible. The different scanning strategies might support the task of model discrimination differently. Intuitively, measuring the concentration close to the diffusion front would be the optimal solution, however, this scanning strategy is not feasible with the device at hand and would require prior knowledge of the position of the diffusion front as a function of time. Simple line and plane scans may give additional information on the exact centering or the roundness of the bead, but this benefit lowers the temporal resolution. For these reasons a single point measurement strategy was followed. Figure 4-5 shows the $\mathrm{pH}$ profiles using the two alternative diffusion laws and different measurement positions. 


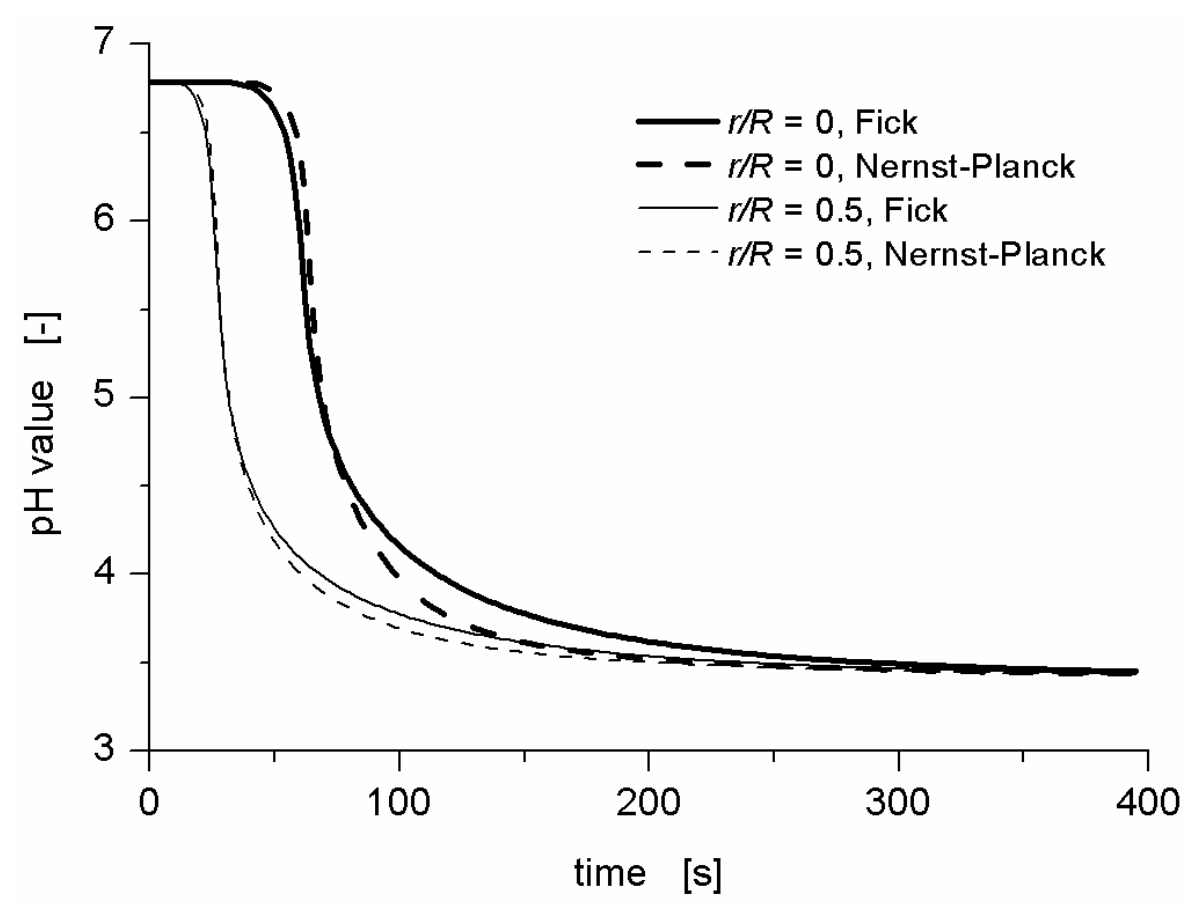

Figure 4-5: $\quad$ Simulation of the development of $\mathrm{pH}$ value over time at two different positions in a hydrogel bead: center of the bead $(\mathrm{r} / R=0)$; middle between the center and the surface of the bead $(\mathrm{r} / R=0.5)$. Fickian and Nernst-Planck diffusion are shown for an initial bulk phase concentration of $100 \mathrm{mM}$ propionic acid into a Ca-alginate hydrogel bead of $R=1.25 \mathrm{~mm}$ radius with an initial $\mathrm{pH}$ value of 6.8 .

The experimental setup poses the following constraints to the optimization problem:

- The radius of the hydrogel bead has an upper limit of $1.25 \mathrm{~mm}$ due to the observable area of the CLSM. On the other hand the preparation of the beads defines a minimal bead radius of $1 \mathrm{~mm}$ in order to guarantee a sufficient roundness.

- The optimal initial $\mathrm{pH}$ value of the hydrogel beads is limited by the choice of resorufin as fluorescent $\mathrm{pH}$ indicator in the experimental setup. The $\mathrm{pH}$ sensitivity covers the range of 4.0 to 7.0 due to an apparent $\mathrm{p} K$ value of resorufin of 5.6 (Figure 4-4). To ensure remaining within this range even in the case of small experimental deviations, the lower bound of the initial $\mathrm{pH}$ value is set to 4.2 and the upper bound to 6.8 .

- The initial total concentration of propionic acid $c_{P a, t o t, 0}$ is limited by the sensitivity range of resorufin. If a too high initial propionic acid concentration is applied, the $\mathrm{pH}$ value drops too fast through the sensitive range, resulting in too few observations. Thus, an upper bound to $c_{P a, t o t, 0}$ is set at $100 \mathrm{mM}$ while the lower bound is set to $0 \mathrm{mM}$. 
The result of the optimization for these experimental degrees of freedom together with the lower and optimal bounds and the initial estimates, is given in Table 4-2.

Table 4-2: Degrees of freedom and results for the optimal experimental design. For the optimization Eq. 4-21 and the optimal experimental design facilities of gPROMS (version 3.0.2, Process System Enterprise Ltd., London, UK) were used.

\begin{tabular}{|c|c|c|c|c|}
\hline degree of freedom & lower bound & upper bound & initial estimate & optimized value \\
\hline measurement position & $0 \cdot R$ & $\mathrm{R}$ & $0.5 \cdot R$ & $0 \cdot R$ \\
\hline bead size & $1 \mathrm{~mm}$ & $1.25 \mathrm{~mm}$ & $1 \mathrm{~mm}$ & $1 \mathrm{~mm}$ \\
\hline initial $\mathrm{pH}$ value & 4.2 & 6.8 & 6.8 & 6.8 \\
\hline $\begin{array}{l}\text { initial concentration of } \\
\text { propionic acid }\end{array}$ & $0 \mathrm{mM}$ & $100 \mathrm{mM}$ & $80 \mathrm{mM}$ & $100 \mathrm{mM}$ \\
\hline
\end{tabular}

The optimal measurement position is in the center of the bead, the optimal bead radius is $1 \mathrm{~mm}$. The initial $\mathrm{pH}$ value within the bead should be as high as possible (6.8) just as the initial total concentration of propionic acid within the bulk, which has an optimal value of $100 \mathrm{mM}$.

\subsubsection{Experimental results}

The diffusion of propionic acid into an Ca-alginate hydrogel bead is observed using the fluorescence lifetime CLSM. The correct particle position is verified by light microscopy after finishing the experiment. At the same time, beads showing irregularities, such as cracks and holes, are rejected. Sixteen sets of experimental data have been obtained using this procedure. One of them is presented in Figure 4-6.

The $\mathrm{pH}$ value in the bead center remains approximately constant for the first 50 seconds. With appearance of the diffusion front in the bead center, a steep drop of the $\mathrm{pH}$ value occurs. Between pH 6.0 and 5.5, a shoulder can be observed, which corresponds to the shoulder in the calibration curve (Figure 4-4). Finally, the equilibrium $\mathrm{pH}$ value of 3.9 is reached. The experimental errors of the $\mathrm{pH}$ value are deduced from the calibration curve (Figure 4-4). 


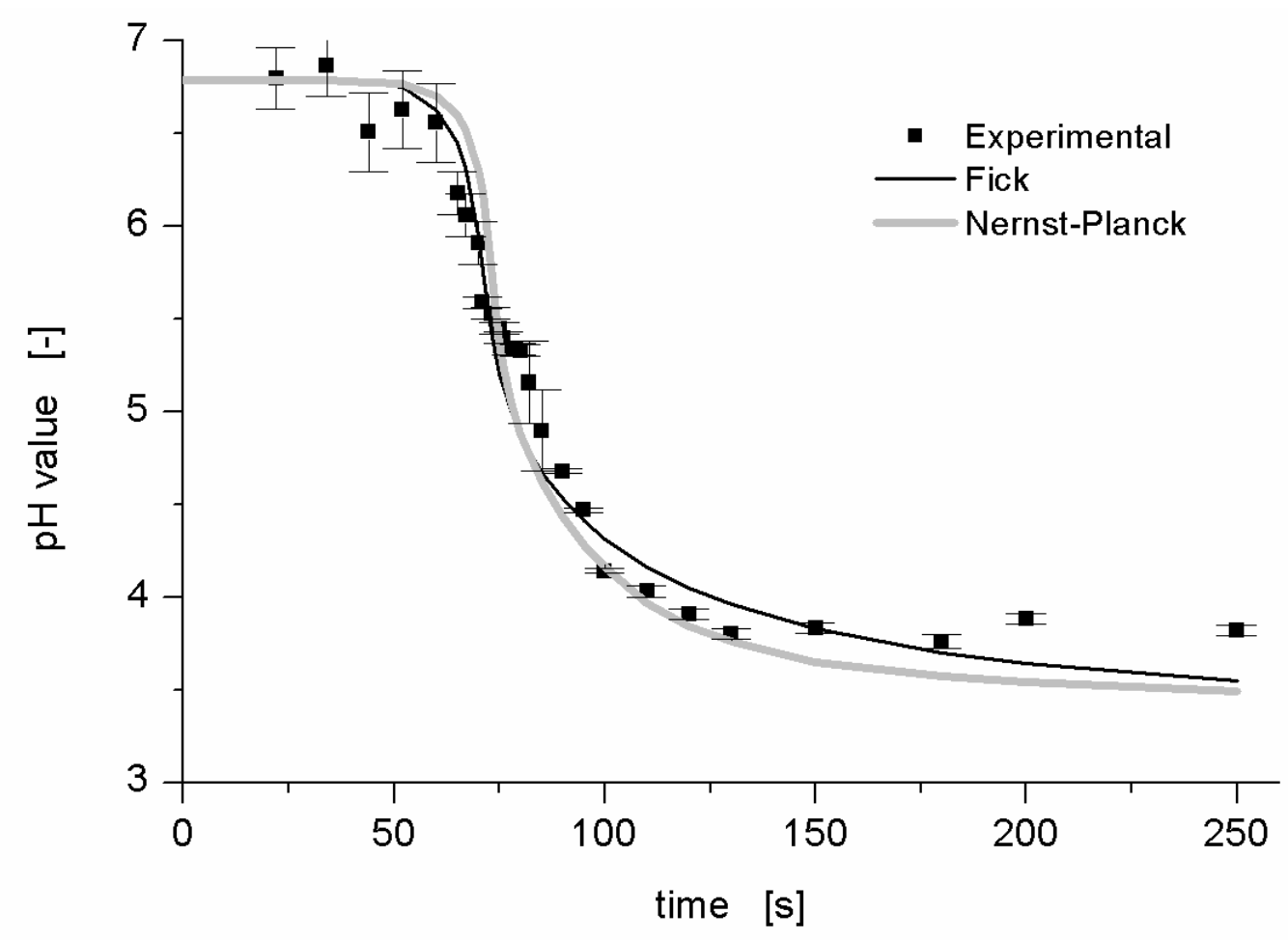

Figure 4-6: $\quad$ Experimental and estimated $\mathrm{pH}$ values in the bead center for the diffusion of propionic acid into Ca-alginate hydrogel beads. The initial model as described in Section 4.1.2 was used $\left(R=1.025 \mathrm{~mm}\right.$, Estimated parameters: $p H_{0}=6.79, c_{P a, t o t, 0}=95 \mathrm{mM}$, alginate influence parameter $\left.k_{F}=5.1 \mathrm{~g} \mathrm{~g}^{-1}, k_{N}=6.6 \mathrm{~g} \mathrm{~g}^{-1}\right)$.

\subsubsection{Parameter estimation and model discrimination}

\subsubsection{First MEXA cycle}

For discriminating the correct diffusion model using the experimental data, i.e. to identify the Fickian or Nernst-Planck diffusion as more appropriate for describing the diffusion of propionic acid in Ca-alginate hydrogel beads, a suitable sequential strategy needs to be defined to determine the model parameters from the experimental data. First the initial experimental values are verified for each individual experiment. This is necessary to account for the inevitable deviations of the experimental conditions from their nominal values. The initial $\mathrm{pH}$ value of the beads is estimated from the first couple of data points. Since equilibrium data depend only on the initial total concentration of propionic acid, this parameter is estimated from the last couple of data points. Finally the bead size is estimated for each individual hydrogel bead within a range of $\pm 2 \%$ around the optically determined Feret diameter. These preliminary estimations of the experimental parameters help to increase 
the precision of the succeeding estimation of the model parameters. Outcome of this are initial $\mathrm{pH}$ values inside the hydrogel bead between $\mathrm{pH}_{0}=6.58$ and 6.76 , initial total concentrations of propionic acid between 95 and $105 \mathrm{mM}$ and bead radii between 1.02 and $1.13 \mathrm{~mm}$.

Finally, all 16 experiments are simultaneously taken into account in order to estimate the matrix interaction parameter $k$ in Eq. 4-15 that accounts for the alginate density influence on the diffusion coefficient. The simulation results for the Fickian and the Nernst-Planck diffusion are demonstrated exemplarily in Figure 4-6. The slope of the $\mathrm{pH}$ decrease over time is less steep for Fickian diffusion in comparison to Nernst-Planck diffusion. The steeper slope for Nernst-Planck diffusion is reproducible for all hydrogel beads. It is attributed to the charge-mediated co-transport of ions resulting in a sharper diffusion front. The evaluation of the objective function for the parameter estimation shows that the value is slightly higher for Nernst-Planck diffusion than for Fickian diffusion, suggesting the latter as the better diffusion law. However, no clear discrimination of both diffusion models is possible.

Although only effective diffusion coefficients, which take the influence of the alginate matrix into account, were estimated and multi-component effects were neglected due to the dilute nature of the system, it was surprising that both diffusion laws are able to describe the diffusion of propionic acid into Ca-alginate hydrogel beads satisfactorily. Nernst-Planck diffusion was expected to be more suitable for modelling electrolyte diffusion in aqueous environments. One potential explanation was that the Ca-alginate hydrogel that consists of polyalginic acids cross-linked by bivalent $\mathrm{Ca}$-ions, might be responsible for a high intrinsic ionic strength that overrules the charge co-transport. An estimation of the ionic strength, however, based on the alginate concentration of $3.3 \%(\mathrm{w} / \mathrm{w})$ alginate gave a local monovalent alginic acid concentration of only $18 \mathrm{mM} . \mathrm{CaCl}_{2}$ was present in concentrations of $180 \mathrm{mM}$, resulting in an ionic strength due to the alginate hydrogel of $I_{A l g} \sim 1 \mathrm{M}$, a high value, but not sufficient to exclude charge interactions. The resulting parameters describing the exponential density influence of the alginate matrix onto the effective diffusion coefficient were in the same order of magnitude for both diffusion models and amounted to $k_{F}=(5.11 \pm 0.06) \mathrm{g} \mathrm{g}^{-1}$ for Fickian diffusion, in contrast to $k_{N}=(6.60 \pm 0.06) \mathrm{g} \mathrm{g}^{-1}$ for Nernst-Planck diffusion, indicating slightly less influence of alginate hydrogel matrix in case of Fickian diffusion. Considering Eq. 4-15 the overall diffusion coefficient in the hydrogel is in the order of $85 \%$ of the one in free solution indicating a strong reduction in comparison with the free diffusion due to the 
hydrogel matrix. A similar reduction was observed by Mateus et al. (1999) for $\kappa$-carrageenan hydrogel beads.

Subsequently, a detailed analysis of the model assumptions was undertaken. Although the investigation of the transport kinetics inside the hydrogel bead was intended for the application of the hydrogel suspended in organic solvents, the experiment was carried out in aqueous environment. This eliminated the need of refractivity index matching and also reduced the model complexity, because no phase equilibria on the phase boundary (Heinemann et al., 2003) had to be taken into account. By eliminating the organic solvent, however, one restriction regarding the mass transfer, i.e. that only uncharged components can cross the phase boundary, was overcome (Eq. 4-4). In this restricted model co-transport of charged species can occur only inside the hydrogel bead, but not through the phase boundary. This restriction leads to the observed similarity of the simulated diffusion process for both Fickian and Nernst-Planck model. If organic solvents would have been used as bulk phase for the diffusion measurements, the presented restricted model should be used, since no cotransport of charged species into the bulk phase would occur. In this case the same results as shown in this Section would be obtained and the diffusion laws could not have been discriminated.

\subsubsection{Second MEXA cycle}

The model was adapted to an aqueous bulk phase by allowing mass transfer across the boundary layer for all species, not only for the uncharged ones. The mass transfer coefficients $k_{\mathrm{i}}$ were related to the respective diffusion coefficients by the Sherwood number $S h$ which is assumed to be 2 in the unstirred case.

First, the unrestricted model was used to simulate the concentrations of charged species both in the bead and in the bulk as shown in Figure 4-7 for chloride ions. Figure 4-7 shows that the chloride concentration decreases significantly in the bead (dimensionless radius $<1$ ), while it increases slightly in the bulk phase (dimensionless radius $>1$ ) due to the large bulk volume. Thus, a diffusion of chloride ions into the bulk phase occurs. Using a qualitative chloride test based on the precipitation of $\mathrm{AgCl}$, the diffusion of chloride ions into the bulk phase could be verified. 


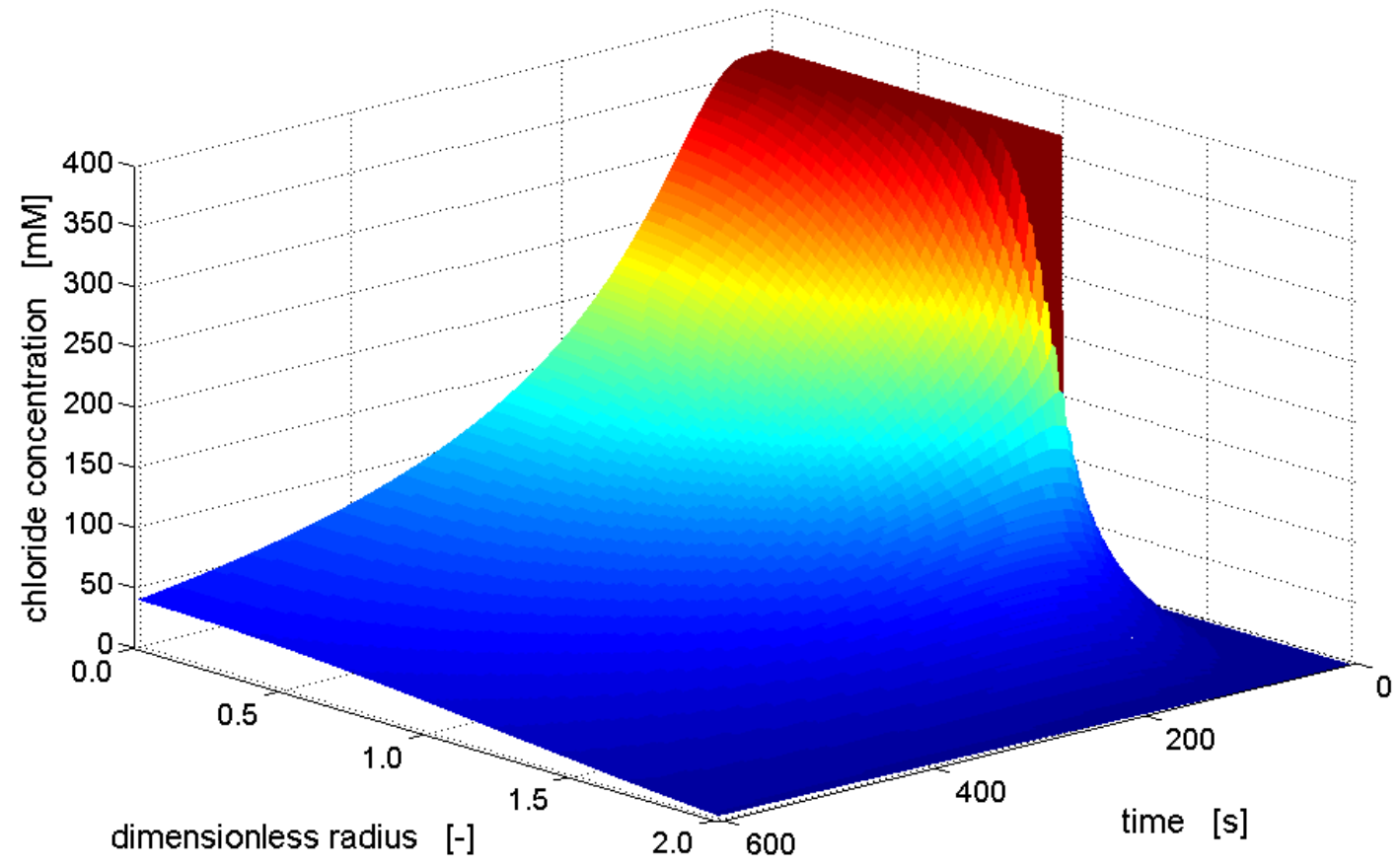

Figure 4-7: Simulation of the chloride concentration in the bead (dimensionless radius $<1$ ) and in the bulk phase (dimensionless radius $>1$ ) using the unrestricted diffusion model and Nernst-Planck law $\left(R=1.025 \mathrm{~mm}, p H_{0}=7.0, c_{P a, t o t, 0}=100 \mathrm{mM}\right)$.

The simulation with the unrestricted model results in a $\mathrm{pH}$ progress curve comparable to the previously simulated curves for Nernst-Planck diffusion. However, the simulation gives a significantly different result for Fickian diffusion, as shown in Figure 4-8.

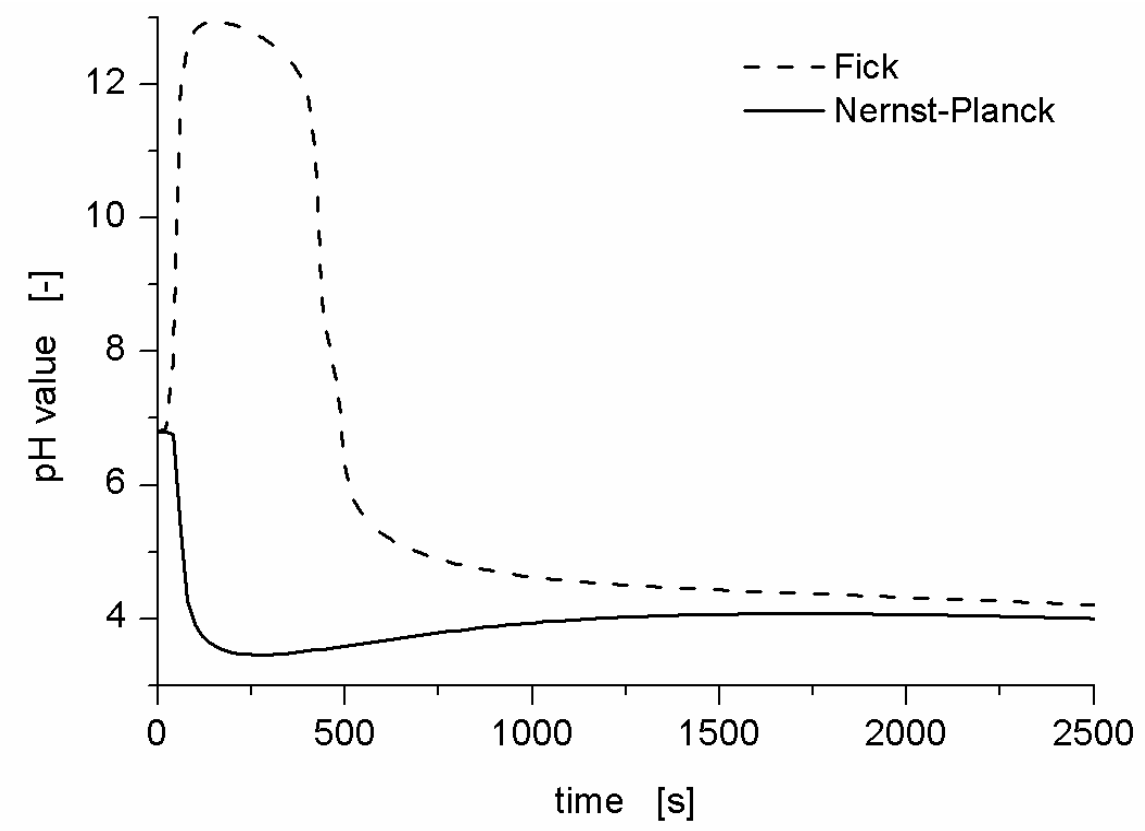

Figure 4-8: $\quad$ Simulated $\mathrm{pH}$ progress in the bead center for Fickian and Nernst-Planck diffusion using the unrestricted model $\left(R=1.005 \mathrm{~mm}, p H_{0}=6.79, c_{P a, t o t, 0}=99.1 \mathrm{mM}, k=0.051\right)$. 
Obviously, Fick's law is no longer capable of describing the experimentally observed diffusion process. The difference of the model predictions can be explained as follows: In the unrestricted model, the diffusion of all species across the phase boundary is possible, resulting in the diffusion of chloride ions from the bead to the bulk as shown in Figure 4-7. Fick's law does not consider the co-transport of charged compounds that enhances or slows down the diffusional transport of other charged species. Therefore, the diffusion rates of all species are independent from each other, if Fick's law is applied. Since chloride ions possess a significantly higher diffusion coefficient than propionic acid (Table 4-1), the diffusion of chloride ions prevails in the beginning. Due to electroneutrality (Eq. 4-12) the decrease of chloride ions is compensated by water dissociation resulting in an apparent disappearance of the strong hydrochloric acid from the bead. Thus, the $\mathrm{pH}$ value increases in the beginning. However, after $400 \mathrm{~s}$ no significant concentration gradient of chloride ions is present any more (Figure 4-7). Then the diffusion of propionic acid prevails which decreases the $\mathrm{pH}$ in the bead again. Since both diffusion model variants affect only the diffusion rate, the same equilibrium value is obtained after long times (Figure 4-8).

The model prediction using Fick's law significantly differs from the measured data. In contrast to this, the Nernst-Planck law takes the co-transport of charged compounds into account, which slows down the diffusion rate of the very mobile chloride ions resulting in a reasonable simulation profile (Figure 4-9). This supports the use of the Nernst-Planck law for electrolyte species.

Using the unrestricted model, the measurement data are, again, explained well by the model. The key difference is found in the estimated parameters. The estimated matrix interaction parameter $k$ that describes the influence of the alginate density on the diffusion coefficient is now estimated two orders of magnitude lower, $k \approx 0.05 \mathrm{~g} \mathrm{~g}^{-1}$, and is statistically no longer significant. Obviously, the alginate matrix does not pose a diffusion barrier to small molecules that serve as typical substrates for industrial fine chemical synthesis using enzymes. This result is in accordance with earlier observations where an alginate influence was observed only for molecules $>20,000$ Da (Tanaka et al., 1984). 


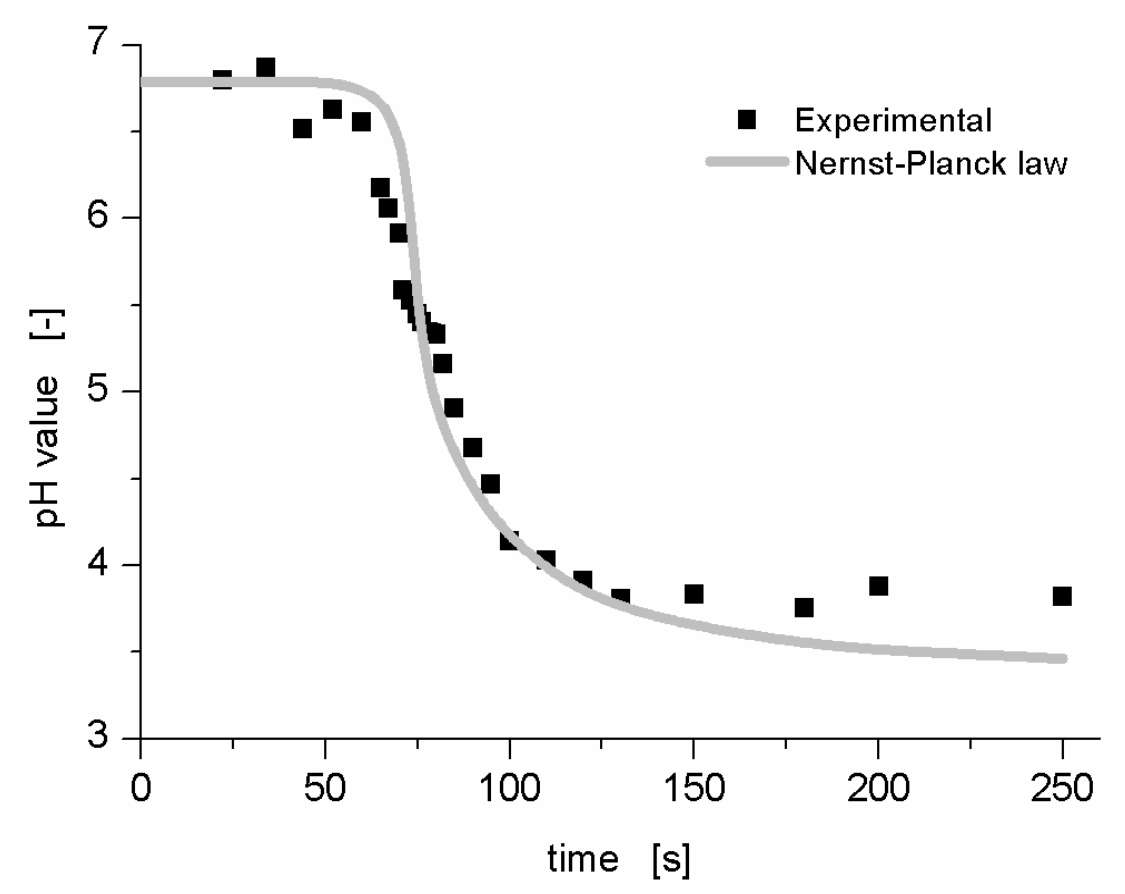

Figure 4-9: $\quad$ Experimental and estimated $\mathrm{pH}$ values over time for the diffusion of propionic acid into Caalginate hydrogel beads using the unrestricted model $\left(R=1.005 \mathrm{~mm}, p H_{0}=6.79\right.$, $\left.c_{P a, t o t, 0}=99.1 \mathrm{mM}, k=0.051\right)$.

Subsequently, the developed model was adapted to the diffusion of butyric acid from an $n$-hexane phase into hydrogel beads consisting of polyvinyl alcohol and polyethylene glycol. The $\mathrm{pH}$ value was determined semi-quantitatively using bromocresol green as $\mathrm{pH}$ indicator. In this case, the experiments were conducted by Nora Bieler (Chair of Biotechnology, RWTH Aachen University). The adapted model is able to predict the experimental data using either Fickian or Nernst-Planck diffusion since no diffusion of charged species into the bulk phase is possible (results not shown), which demonstrates the general applicability of the developed model for the rational design of hydrogel beads.

\subsubsection{Conclusions}

The objective of this work was the discrimination of diffusion laws for the description of propionic acid diffusion from a bulk phase into Ca-alginate hydrogel beads. This simple model system was chosen in order to demonstrate steps towards a rational design of biocatalysis using hydrogels in organic solvents. Fluorescence lifetime CLSM was used for the quantification of dynamic $\mathrm{pH}$ changes in macroscopically large particles. As experimental setup, the diffusion from the bulk solution into a hydrogel bead fixed on a circular holder in a microtiter plate well was chosen that allows a fast screening of hydrogel variants. A kinetic 
model was developed describing the diffusion process. In case of diffusion from an aqueous bulk, Fick's law could not adequately describe the experimentally obtained data, in contrast to the Nernst-Planck diffusion model. For small molecules, the Ca-alginate hydrogel matrix had no influence on the effective diffusion coefficient, which is equal to the diffusion coefficient in a liquid system.

In parallel to the investigation of the diffusion of propionic acid, this Section also illustrates the use of model-based experimental analysis (MEXA). Therefore, one detour made during the modelling process was shown that demonstrates that Fickian and Nernst-Planck diffusion models cannot be discriminated in the original hydrogel/organic solvent system, but only using an aqueous bulk solution. In this way the potential of a structured work process to systematically identify suitable models was demonstrated.

\subsection{Diffusion in k-carrageenan hydrogel beads}

\subsubsection{Introduction}

To investigate the gel-stabilized biphasic system with immobilized benzaldehyde lyase $(\mathrm{BAL})$, the diffusion rates of the reactants 3,5-dimethoxy-benzaldehyde (DMBA) and $(R)$ 3,3',5,5'-tetramethoxy-benzoin need to be quantified previously. Since Ca-alginate beads exhibit an inhomogeneous density distribution (Heinemann et al., 2005), $\kappa$-carrageenan is chosen as hydrogel material. In contrast to Ca-alginate, the $\kappa$-carrageenan hydrogel beads are produced by cryogelation, which results in a homogeneous density distribution. Similar to the investigation of the propionic acid diffusion (Section 4.1), the model-based experimental analysis (MEXA) work process is applied.

\subsubsection{Model development}

For modeling the diffusion of DMBA and TMB the diffusion model developed in Section 4.1.2 is used. However, the model can be simplified due to the following reasons:

- There are several physical models which aim to describe the matrix interaction of a polymer on the diffusion rate of solutes (Cukier, 1984; Gao and Fagerness, 1995; Masaro 
and Zhu, 1999; Muhr and Blanshard, 1982). However, for DMBA and TMB the diffusion coefficients in bulk liquid are not available which is a prerequisite to quantify the matrix interaction. Instead effective diffusion coefficients for DMBA and TMB are used.

- Due to the different gelation method used $\kappa$-carrageenan beads possess a homogeneous density distribution in contrast to Ca-alginate (Skjak-Braek et al., 1989). For this reason, Eqs. 4-14-17 can be omitted.

- DMBA and TMB are uncharged substances. For this reason, the second summand of the Nernst-Planck law (Eq. 4-8) equals zero, resulting in Fick's law (Eq. 4-9).

- Since DMBA and TMB do not dissociate the diffusion rates of DMBA and TMB are assumed to be independent from other species. The diffusion model can be reduced to the model equations for DMBA and TMB. Therefore, Eqs. 4-10-13 can also be omitted.

The following model equations remain:

$$
\begin{gathered}
V_{\text {bulk }} \frac{d c_{i, \text { bulk }}}{d t}=k_{i} \cdot A_{\text {bead }} \cdot\left(c_{i, \text { bead }}(r=R)-c_{i, \text { bulk }}\right) \\
\frac{\partial c_{i, \text { bead }}}{\partial t}=-\frac{1}{r^{2}}\left(\frac{\partial}{\partial r} \cdot r^{2} \cdot j_{i}\right) \\
j_{i}=-D_{i, \text { eff }} \cdot \frac{\partial c_{i}}{\partial r} \\
j_{i}(r=0)=0 \\
j_{i}(r=R)=k_{i} \cdot\left(c_{i, \text { bead }}(r=R)-c_{i, \text { bulk }}\right) \\
c_{i, \text { bulk }}(t=0)=c_{i, \text { bulk }, 0} \\
c_{i, \text { bead }}(t=0)=c_{i, \text { bead }, 0}
\end{gathered}
$$




\subsubsection{Sensitivity analysis}

The purpose of the diffusion experiments is to determine the diffusion coefficient of the reactants precisely. In order to decide for the optimal experimental strategy, a sensitivity analysis is performed prior to the experiments. Two strategies are compared in Figure 4-10 concentration measurements in the bulk phase and in the bead center. The first strategy has already been applied by Mateus et al. (1999) to $\kappa$-carrageenan hydrogel beads.
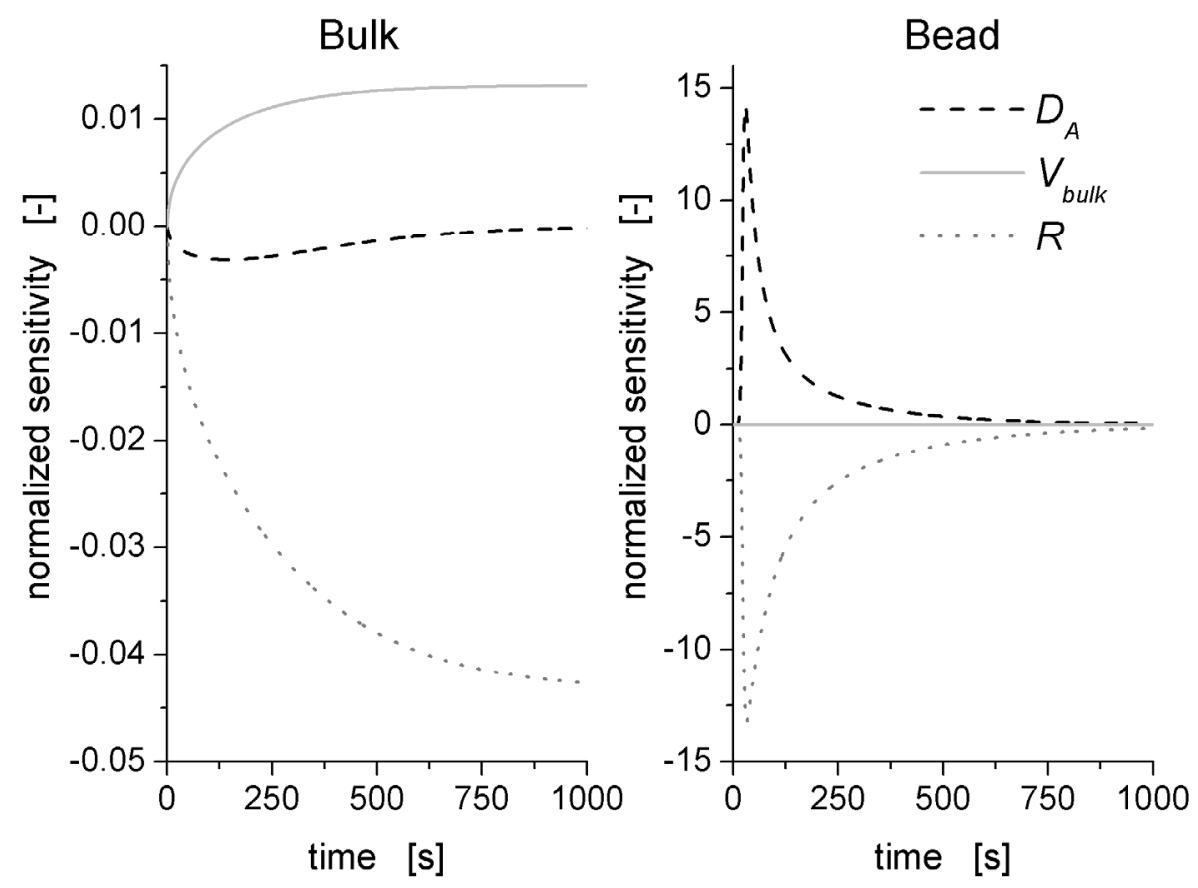

Figure 4-10: Sensitivity analysis for the diffusion coefficient of DMBA $D_{A}$, the bulk volume $V_{b u l k}$, and the bead radius $R$ in the bulk phase (left) and in the bead center (right). The sensitivity refers to the DMBA concentration. The sensitivities were normalized as shown in Chapter 4.1.4 $\left(c_{A, b u l k, 0}=50 \mathrm{mM}, D_{A}=5.57 \cdot 10^{-10} \mathrm{~m}^{2} \mathrm{~s}^{-1}, R=1 \mathrm{~mm}, V_{\text {bulk }}=300 \mu \mathrm{L}\right)$.

Figure 4-10 shows that the normalized sensitivity of the diffusion coefficient of DMBA is more than three orders of magnitude higher in the bead center. This means that the precision of a parameter estimate will be much higher, if concentrations are measured in the bead compared to bulk measurements. Therefore, an optical measurement technique is necessary. In the previous Section 4.1, fluorescence lifetime confocal laser scanning microscopy (CLSM) is applied to measure the $\mathrm{pH}$ value using a $\mathrm{pH}$ indicator. An alternative to this indirect method is two photon LSM (Denk et al., 1990), which is used for the following investigations. Since DMBA and TMB are fluorophores, their concentrations can be directly measured and no fluorescent markers are required. To excite one fluorophore molecule, it has 
to be hit by two photons concurrently. Only in the focal point of the fluorescence excitation beam the probability of a two photon event is high enough to achieve a fluorescence emission. Thus, an excellent spatial resolution can be obtained.

Figure 4-10 demonstrates another advantage of measurements in the bead: Inaccuracies in the added bulk volume have only an insignificant influence on the DMBA concentration in the bead center. Thus, inevitable minor pipetting errors do not affect the estimate of the diffusion coefficient. On the contrary, if bulk measurements are carried out, these pipetting errors can distort the estimate dramatically. However, for both experimental techniques the bead radius significantly affects the measured concentrations. Therefore, the bead radius has to be accurately determined prior to the estimation of the diffusion coefficient.

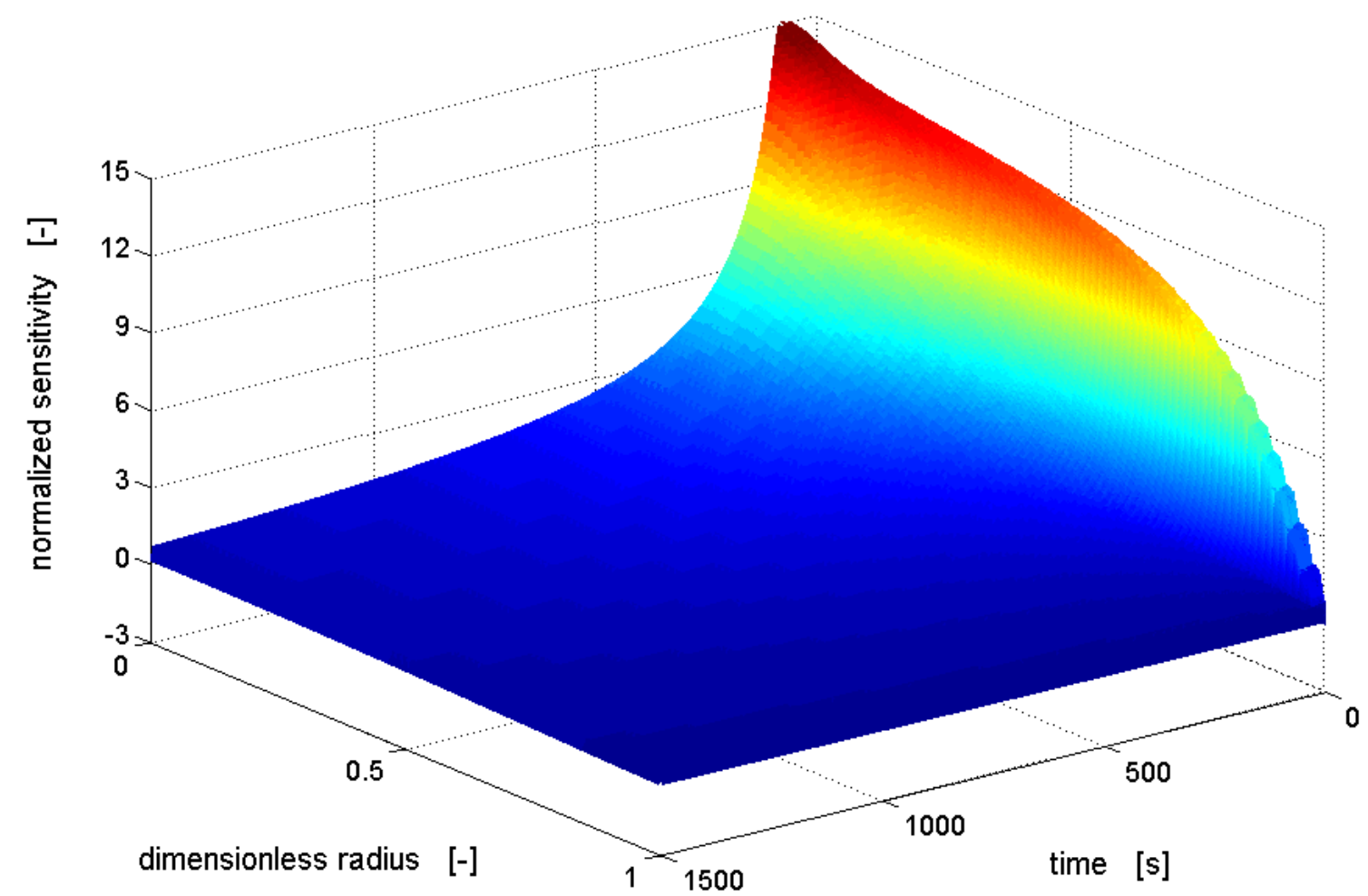

Figure 4-11: $\quad$ Normalized sensitivity of the diffusion coefficient $D_{A}$ inside the hydrogel bead. The sensitivity refers to the DMBA concentration. The sensitivity was normalized as shown in Chapter 4.1.4 $\left(c_{A, b u l k, 0}=50 \mathrm{mM}, D_{A}=5.57 \cdot 10^{-10} \mathrm{~m}^{2} \mathrm{~s}^{-1}, R=1 \mathrm{~mm}, V_{\text {bulk }}=300 \mu \mathrm{L}\right)$.

To determine the optimal measuring position for the two photon LSM, the sensitivities in the whole hydrogel bead are analyzed. Figure 4-11 confirms that the bead center is the optimal measuring position since the highest normalized sensitivity is reached there. The reason is that 
in this way the diffusion distance is maximized. Although the absolute values differ, the same results are obtained for TMB (results not shown).

\subsubsection{Experimental methods}

The cosolvent dimethylformamide (DMF) was obtained from Fluka (Neu-Ulm, Germany), all other chemical substances were purchased from Sigma-Aldrich (Deisenhofen, Germany). All chemicals used were of analytical grade.

$\kappa$-carrageenan hydrogel beads were prepared by adding $1.5 \%(\mathrm{w} / \mathrm{v})$ carrageenan to $50 \mathrm{mM}$ $\mathrm{KH}_{2} \mathrm{PO}_{4} / \mathrm{K}_{2} \mathrm{HPO}_{4}$ buffer with a $\mathrm{pH}$ value of 8.5 . The suspension was heated up to $90^{\circ} \mathrm{C}$ in order to dissolve the carrageenan. Afterwards, the solution was cooled down to $45^{\circ} \mathrm{C}$ where it remains liquid. Using a pipette $15 \mu \mathrm{L}$ of the solution were dropped into a column containing vegetable oil. The column was heated at the top to $45^{\circ} \mathrm{C}$ and cooled at the bottom to $0^{\circ} \mathrm{C}$, thus, a temperature gradient was achieved. Due to the minimization of the interface round drops were obtained which harden to hydrogel beads by cryogelation. The hydrogel beads

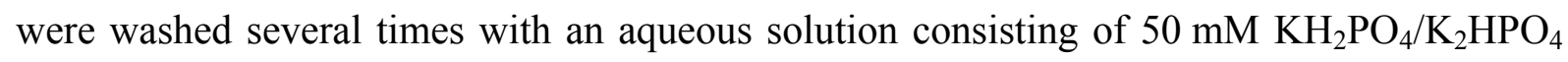
and $25 \%(\mathrm{v} / \mathrm{v}) \mathrm{DMF}$.

As done in the previous Section, the diffusion experiments were performed in a 96-well microtiter plate at room temperature. The hydrogel bead was placed on top of a circular holder to guarantee uniform conditions. The diffusion experiments were started by the addition of $300 \mu \mathrm{L}$ of an aqueous DMBA solution to the well. This aqueous solution contains $50 \mathrm{mM} \mathrm{KH} \mathrm{PO}_{4} / \mathrm{K}_{2} \mathrm{HPO}_{4}$ buffer ( $\mathrm{pH} 8.5$ ), and 25\%(v/v) DMF. Due to the short duration of the experiment solvent evaporation was negligible.

After each measurement, the correct position and the quality of the hydrogel bead were controlled using light microscopy. If they were not satisfactory, the respective data were not considered. According to the results of the sensitivity analysis, the bead radius was measured with the device depicted in Figure 4-3 (Buthe et al., 2004). This also allows the determination of the roundness $\left(R_{\min } / R_{\max }\right)$. Beads with a roundness less than 0.8 were rejected.

The two photon LSM experiments were conducted using the flexible Ti-sapphire oscillator Mai Tai HP laser system (Spectra Physics, Mountain View, CA, USA). This system allows 
emission wavelengths between $690 \mathrm{~nm}$ and $1020 \mathrm{~nm}$, with an average power between 0.04 and $3 \mathrm{~W}$, depending on the wavelength. The repetition rate is $80 \mathrm{MHz}$ and the pulse duration is below $100 \mathrm{fs}$. A prism compressor prevents the broadening of the laser pulses. The excitation wavelength for both DMBA and TMB was $723 \mathrm{~nm}$, which is approximately the doubled wavelength of their excitation maximum due to the two photon process. In both cases all photons with a wavelength below the excitation wavelength were collected by a photomultiplier (PMT) with high quantum efficiency and accurate timing characteristics (H7422P-40, Hamamatsu Photonics GmbH, Herrsching, Germany). The electrical pulses from the PMT are guided to an external PC system that writes the results to a file.

For calibration hydrogel beads were equilibrated with different concentrations of DMBA and TMB, respectively. Using the measured fluorescence intensities, linear calibration curves were obtained (Eq. 4-29), where $a$ is a calibration parameter. Since the measuring technique is very sensitive, for each measurement series a new calibration curve was determined.

$$
\text { Int }=a \cdot c_{i}
$$

\subsubsection{Experimental results and parameter estimation}

To estimate the diffusion coefficient of DMBA and TMB the diffusion model was fitted to 27 experiments, respectively. The quality of the experimentally obtained diffusion data is quite diverse. Experiments conducted under identical initial conditions can deviate significantly from each other. The reasons for this are the difficulty in determination of the starting time, deviations of bead sizes, and errors in the initial concentrations. Thus, a sequential parameter estimation approach was applied. First, besides the diffusion and mass transfer coefficient, which were estimated globally, three additional parameters were estimated individually for each experiment. The parameter lag-time, which allows the estimation of the starting time, was estimated with an interval of $10 \mathrm{~s}$ around the nominal starting time. The bead sizes and the initial bulk concentrations were estimated in bounds of $\pm 10 \%$. Probably due to impurities some experimental data show a base-line not equal to zero. Therefore, a base-line correction was made for these experiments. After these corrections a final parameter estimation of the diffusion and mass transfer coefficient was performed. 


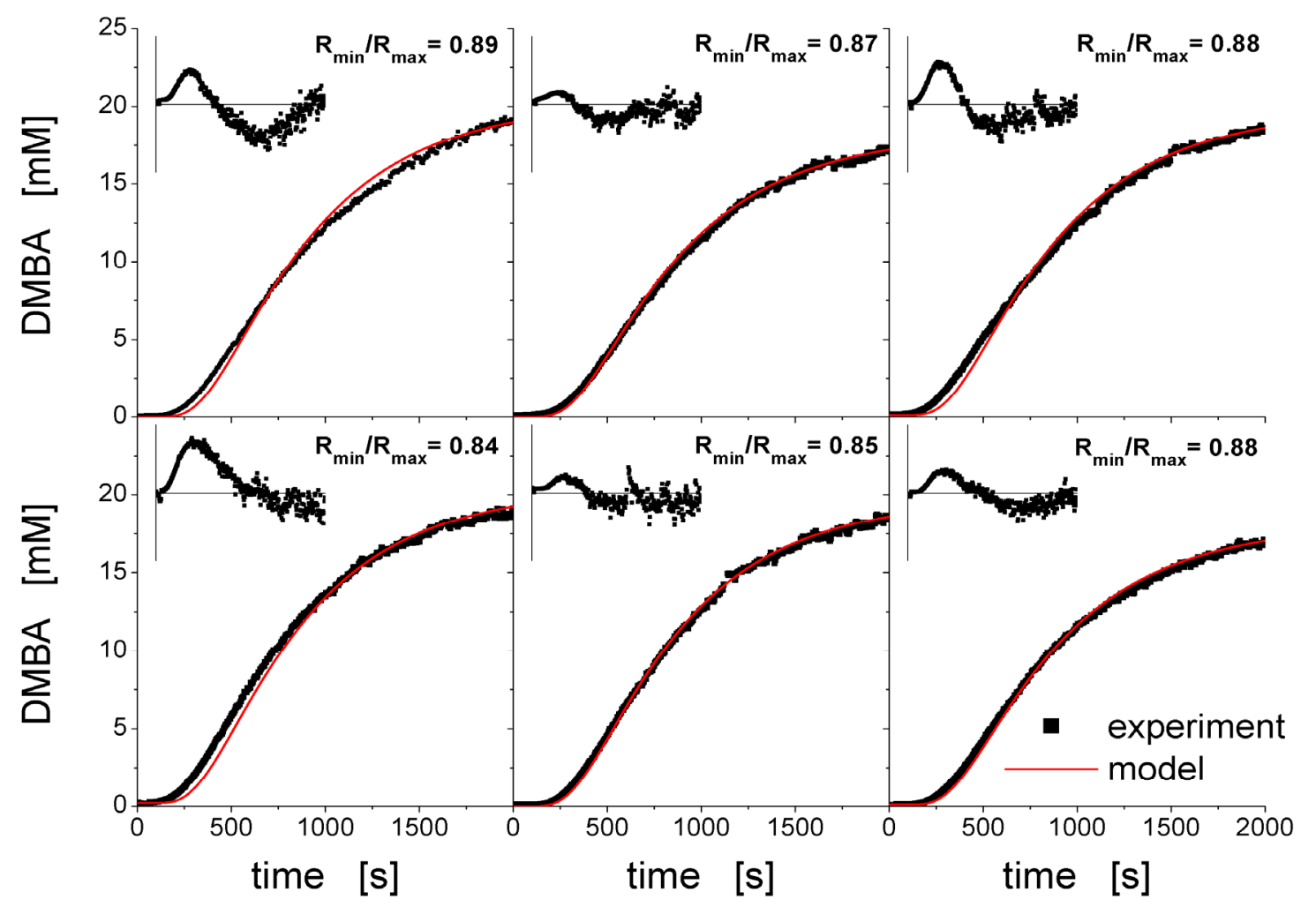

Figure 4-12: Comparison of the fitted diffusion model with the experimental data for DMBA diffusion. 6 of 27 fits are shown as example. The roundness of each bead is provided and the residuals are shown as small inlets (nominal conditions: $c_{A, b u l k, 0}=25 \mathrm{mM}, R=1.5 \mathrm{~mm}$ ).

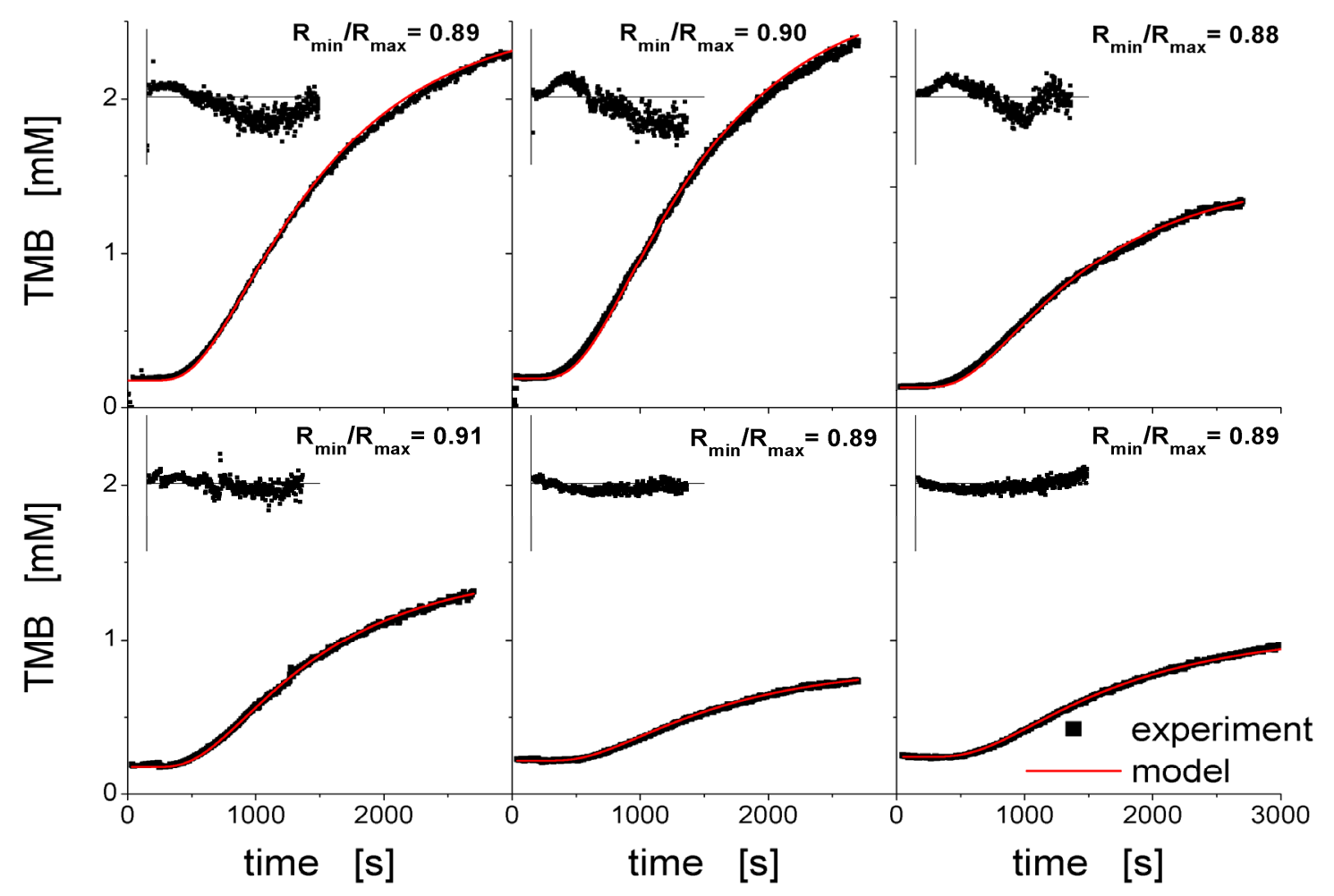

Figure 4-13: Comparison of the fitted diffusion model with the experimental data for TMB diffusion. 6 of 27 fits are shown as example. The roundness of each bead is provided and the residuals are shown as small inlets (nominal conditions: $c_{P, b u l k, 0}=1.5-2.5 \mathrm{mM}, R=1.5 \mathrm{~mm}$ ). 
Figures 4-12 and 4-13 show the fit of the diffusion model to experimental data. For most experiments the residuals exhibit a clear trend which indicates a systematic deviation between model and experimental data. Obviously, the model usually underestimates the concentration of the diffusing species in the beginning, when the diffusion front reaches the bead center. This can be explained as follows: The diffusion model is based on the assumption of ideally spherical beads. In practice, however, the beads are not perfectly round, which reduces the minimal distance between bead surface and bead center (Figure 4-14). Therefore, the diffusing species reaches the bead center earlier than in case of an ideally spherical bead. Thus, the experimental data increase earlier than the model predicts. The deviation increases with decreasing roundness.

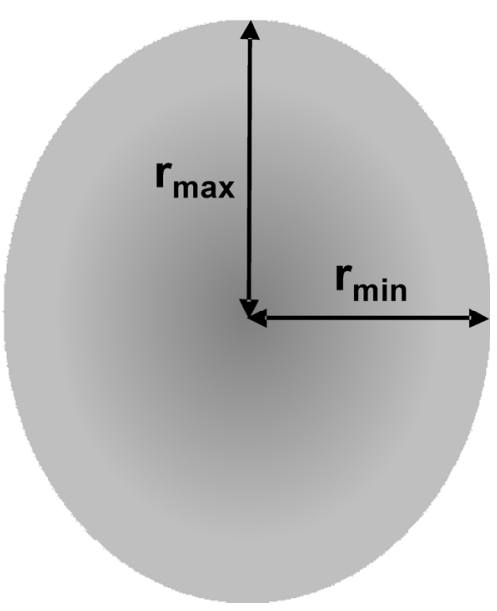

Figure 4-14: $\quad$ Illustration of unround beads (exaggerated for visualization).

Despite the systematic deviations, the diffusion coefficients can be estimated with high precision (Table 4-3), whereas no reliable values for the mass transfer coefficients $k_{i}$ at the bead surface can be obtained (always the upper bound is hit). This indicates a diffusion limitation. If the values for the mass transfer coefficients are decreased to a level where external mass transfer becomes limiting, the resulting curves show totally different behaviors and the model can no longer be fitted to the experimental data. This supports the finding that the system is diffusion-limited. The diffusion coefficient of TMB is lower due to its larger molecular volume. The ratio of the diffusion coefficients of DMBA and TMB is 1.9. This is in good agreement with the ratio of the mass transfer coefficients, which were determined in Chapter 3, of 1.8. The slight difference is probably caused by the hydrogel matrix, which possibly obstructs the diffusion of larger substances like TMB to a larger extent. 
Table 4-3: $\quad$ Estimated effective diffusion coefficients of DMBA and TMB in $\kappa$-carrageenan hydrogel beads.

\begin{tabular}{llll}
\hline parameter & estimate & standard deviation & unit \\
\hline$D_{A, \text { eff }}$ & $4.139 \cdot 10^{-10}$ & $3.881 \cdot 10^{-13}$ & $\mathrm{~m}^{2} \mathrm{~s}^{-1}$ \\
$D_{P, \text { eff }}$ & $2.261 \cdot 10^{-10}$ & $6.197 \cdot 10^{-13}$ & $\mathrm{~m}^{2} \mathrm{~s}^{-1}$ \\
\hline
\end{tabular}

\subsubsection{Conclusions}

For the determination of the diffusion coefficients of DMBA and TMB in $\kappa$-carrageenan hydrogel beads the MEXA work process was applied. Sensitivity analysis revealed that the optimal measuring position is the bead center. Therefore, two photon LSM was applied to quantify the concentration of the fluorescent substances. The diffusion coefficients of both substances could be estimated with high precision. The ratio of the diffusion coefficients is in good accordance with the ratio of the previously determined mass transfer coefficients.

\subsection{Diffusion in K-carrageenan hydrogel cylinders}

\subsubsection{Introduction}

In the first two Sections of this chapter, confocal laser scanning microscopy was applied to investigate the diffusion in hydrogels. In this Section nuclear magnetic resonance (NMR) is investigated as an alternative measurement technique. NMR has the advantages that any chemical species can be observed and does not require any probes or isotopic labelling. Moreover, diffusion measurements of different components can be carried out concurrently. NMR requires only small samples and is non-invasive (Malmborg et al., 2003). NMR takes advantage of the magnetic properties called spins of protons and neutrons in the nucleus. Most common is ${ }^{1} \mathrm{H}$ NMR, which makes use of the spins of single protons. In an ${ }^{1} \mathrm{H}$ NMR spectrum the peak integral is proportional to the amount of protons of the same resonance frequency. 
NMR diffusometry is based on the measurement of the spatial displacement of spins over time. For the NMR signal to be encoded for position, magnetic field gradient pulses of a specific length are used (Fukushima, 1999). The relative peak integral of a particular proton at a specific position in the sample will change over time and this change is attributed to the displacement of the molecules due to diffusion. The peak integral can be converted to the absolute molar concentration by using a solute of a known concentration as reference (Salvati et al., 2007). The challenge of measuring diffusion in a complex medium like the $\kappa$-carrageenan hydrogel is assigning the correct peak to evaluate the concentration profile. This difficulty is due to overlapping peaks of ${ }^{1} \mathrm{H}$ NMR spectrum with that of the medium. To this end, a preliminary measurement must be made in order to locate which peak shows a visible intensity change over time. This peak will then be labeled for the evaluation of concentration profiles in subsequent measurements.

NMR diffusometry has previously been applied to measure inter-diffusion coefficients in polymer solutions and gels (Malmborg et al., 2003; Kwak et al., 2003; Salvati et al., 2007; Seland et al., 2003). However, no similar studies have been performed in $\kappa$-carrageenan hydrogel so far. For this reason, preliminary investigations of the diffusion of DMBA in $\kappa$-carrageenan using NMR are performed in this study. Moreover, the current challenges of NMR diffusometry will be discussed on the basis of this example.

\subsubsection{Materials and methods}

The applied NMR device is able to measure concentrations at different axial positions. Since the concentrations in one axial plane are averaged, hydrogel beads which require radial measurements cannot be employed. Thus, hydrogel cylinders are used instead. The experimental setup for measuring the diffusion of DMBA is illustrated in Figure 4-15.

The $\kappa$-carrageenan hydrogel was prepared as described in Section 4.2.4 with the difference that cryogelation of the gel is directly performed in the diffusion cell. DMBA was dissolved in deuterated water $\left(\mathrm{D}_{2} \mathrm{O}\right)$ without any cosolvent in order to avoid overlapping of the ${ }^{1} \mathrm{H}$ NMR spectra of water or dimethylformamide (DMF) with that of DMBA. The diffusion experiment was initiated by pipetting the DMBA solution into the diffusion cell. 


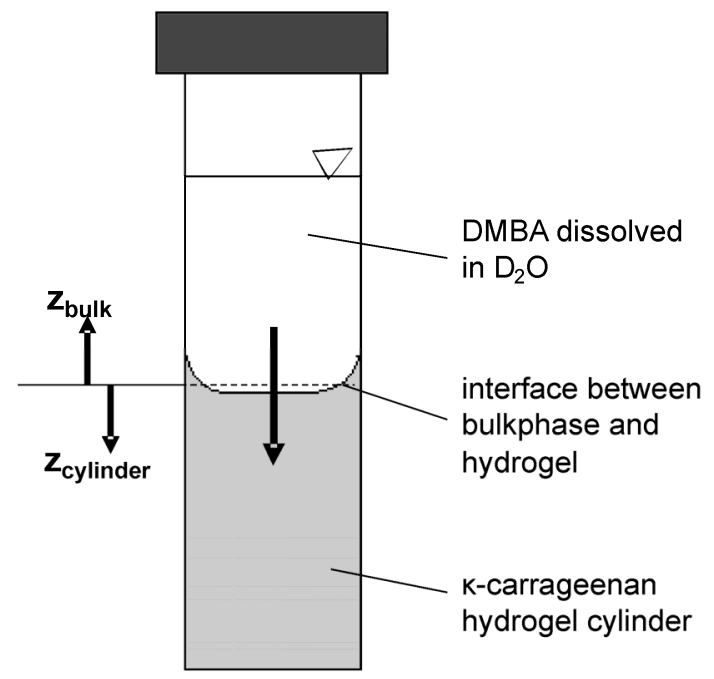

Figure 4-15: The diffusion cell consisting of a hydrogel phase below a bulk phase. Direction of DMBA diffusion is indicated with the arrow. The coordinate system in each phase is labeled as $z_{\text {bulk }}$ and $z_{\text {cylinder }}$.

The NMR experiments were conducted at $11.7 \mathrm{~T}$ field strength $\left(500 \mathrm{Mhz}{ }^{1} \mathrm{H}\right.$ Larmor frequency) with a Bruker DSX 500 spectrometer. Standard Bruker microimaging hardware was used with birdcage resonators of 10 and $25 \mathrm{~mm}$ inner diameter. The microimaging unit provided a maximum gradient strength of $1.0 \mathrm{~T} \mathrm{~m}^{-1}$. One axial profile can be measured within one hour. Due to the limited length of the radio frequency coil of the NMR device the axial profile can be measured within a range of only $2 \mathrm{~cm}$. The axial resolution is $0.8 \mathrm{~mm}$. The experiments were carried out by Andrea Amar (Chair of Macromolecular Chemistry, RWTH Aachen, University).

\subsubsection{Model development}

Preliminary investigations have shown that the bulk phase cannot be considered as ideally mixed. Thus, diffusion of DMBA in the bulk phase has to be taken into account. Assuming a horizontal interface and neglecting evaporation and swelling of the hydrogel, the following diffusion model for hydrogel cylinders was developed:

$$
\begin{gathered}
\frac{\partial c_{i, \text { bulk }}}{\partial t}=-\frac{\partial j_{i, \text { bulk }}}{\partial \mathbf{z}_{\text {bulk }}} \\
\frac{\partial c_{i, \text { cylinder }}}{\partial t}=-\frac{\partial j_{i, c y l i n d e r}}{\partial \mathbf{z}_{\text {cylinder }}}
\end{gathered}
$$




$$
\begin{gathered}
j_{i, \text { bulk }}=-D_{i} \cdot \frac{\partial c_{i, \text { bulk }}}{\partial \mathbf{z}_{\text {bulk }}} \\
j_{i, \text { cylinder }}=-D_{i, \text { gel }} \cdot \frac{\partial c_{i, \text { cylinder }}}{\partial \mathbf{z}_{\text {cylinder }}}
\end{gathered}
$$

In these equations $j_{i}$ denotes the molar diffusive flux, $c_{i}$ the concentrations, and $D_{i}$ and $D_{i, g e l}$ the effective diffusion coefficient in the bulk liquid and in the hydrogel, respectively. The following initial and boundary conditions are added to the model, where $L_{\text {bulk }}$ and $L_{c y l i n d e r}$ denote the lengths of the bulk phase and of the hydrogel cylinder, respectively:

$$
\begin{gathered}
c_{i, \text { bulk }}(t=0)=c_{i, \text { bulk }, 0} \\
c_{i, \text { cylinder }}(t=0)=0 \\
j_{i, \text { bulk }}\left(z_{\text {bulk }}=L_{\text {bulk }}\right)=0 \\
j_{i, \text { cylinder }}\left(z_{\text {cylinder }}=L_{\text {cylinder }}\right)=0 \\
c_{i, \text { bulk }}\left(z_{\text {bulk }}=0\right)=c_{i, \text { cylinder }}\left(z_{\text {cylinder }}=0\right) \\
j_{i, \text { bulk }}\left(z_{\text {bulk }}=0\right)=-j_{i, c y l i n d e r}\left(z_{\text {cylinder }}=0\right)
\end{gathered}
$$

\subsubsection{Results and discussion}

Since the NMR diffusion experiments last about 20 hours, are very laborious, and only few experiments can be conducted, it is important to maximize the information content of each experiment by using optimal experimental design (Bardow and Marquardt, 2007; Walter and Pronzato, 1990). Thus, an optimal diffusion experiment was designed using the D-criterion (Table 4-4). The D-criterion minimizes the determinant of the variance-covariance matrix, which can be geometrically interpreted as minimizing the volume of a confidence ellipsoid (Asprey and Macchietto, 2000; Walter and Pronzato, 1990). 
Table 4-4: Optimal experimental design for a diffusion experiment in hydrogel cylinders. Degrees of freedom were the initial DMBA concentration in the bulk phase $c_{A, b u l k, 0}(0-25 \mathrm{mM})$, the length of bulk phase $L_{\text {bulk }}(0-0.15 \mathrm{~m})$, and the duration $t_{\exp }(1-20 \mathrm{~h})$. The length of the cylinder $L_{c y l i n d e r}$ was fixed to $0.05 \mathrm{~m}$.

\begin{tabular}{lll}
\hline degree of freedom & optimized value & unit \\
\hline$c_{A, \text { bulk, }}$ & 25 & $\mathrm{mM}$ \\
$L_{\text {bulk }}$ & 0.015 & $\mathrm{~m}$ \\
$t_{\text {exp }}$ & 20 & $\mathrm{~h}$ \\
\hline
\end{tabular}

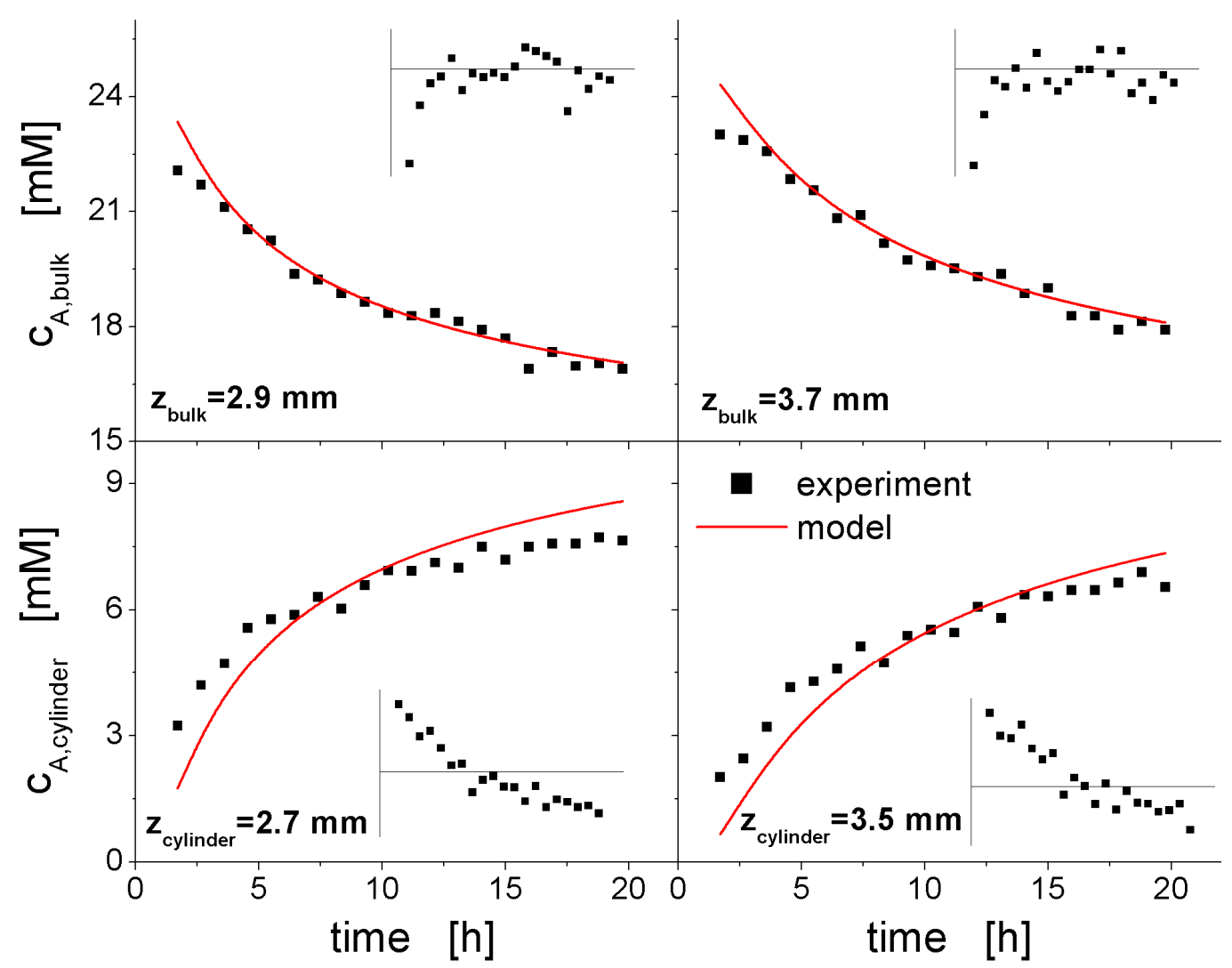

Figure 4-16: Comparison of the fitted diffusion model with the experimental data for DMBA diffusion using ${ }^{1} \mathrm{H}$ NMR. The residuals are shown as small inlets. The optimized experimental conditions listed in Table 4-4 were applied.

The fit of the kinetic model to the experimental data is shown in Figure 4-16. In comparison to the two photon LSM results (Figure 4-12) less data points are obtained. Moreover, the residuals indicate systematic deviations between model and experimental data. However, the diffusion coefficients of DMBA in the bulk phase and in the hydrogel can be estimated precisely (Table 4-5). The correlation between them is very low (-0.05). The diffusion coefficient in the hydrogel is $15 \%$ lower than in the bulk phase. One explanation for this 
could be a hindrance due to the hydrogel matrix. However, it is also possible that minor vibrations inside the apparatus cause an additional mixing of the bulk phase which would be expressed in a higher apparent diffusion coefficient in the bulk.

Table 4-5: Estimated diffusion coefficients of DMBA in the $\mathrm{D}_{2} \mathrm{O}$ bulk phase and in $\kappa$-carrageenan hydrogel.

\begin{tabular}{llll}
\hline parameter & estimate & standard deviation & unit \\
\hline$D_{A, \text { bulk }}$ & $2.892 \cdot 10^{-10}$ & $3.033 \cdot 10^{-12}$ & $\mathrm{~m}^{2} \mathrm{~s}^{-1}$ \\
$D_{P, \text { gel }}$ & $2.465 \cdot 10^{-10}$ & $2.250 \cdot 10^{-12}$ & $\mathrm{~m}^{2} \mathrm{~s}^{-1}$ \\
\hline
\end{tabular}

The diffusion coefficient in the hydrogel is $40 \%$ lower than the value previously obtained with two photon LSM in hydrogel beads (Table 4-3). One explanation could be that the presence of the cosolvent DMF lowers the viscosity $\eta$ (at $20^{\circ} \mathrm{C}: \eta_{D M F}=0.8 \mathrm{mPa} \mathrm{s}$, $\eta_{H 2 O}=1.0 \mathrm{mPa} \mathrm{s}$ ). The lower viscosity results in a higher diffusion coefficient according to the Stokes-Einstein equation (Eq. 4-40), where $k_{B}$ is the Boltzmann constant, $T$ the absolute temperature, and $r$ the radius of the molecule.

$$
D=\frac{k_{B} \cdot T}{6 \cdot \pi \cdot \eta \cdot r}
$$

However, absence of DMF cannot be the only reason for the lower value. Several drawbacks of NMR were observed. First, the quantification of concentrations using NMR spectra might be imprecise. Second, the experiments last longer and provide data with a lower spatial and temporal resolution in comparison to CLSM. The fit using the NMR experiment is based on one single experiment with in total 240 data points. For the fit to the CLSM data 27 experiments were conducted, each containing 400 data points. Thus, the estimate using CLSM is based on 10,800 data points, 45 times more than for the estimate using NMR. Therefore, the CLSM results are considered to be more reliable. Third, due to the longer duration of each experiment evaporation and swelling of the hydrogel can become significant. In future experiments evaporation should be suppressed or at least its rate should be quantified, in order to consider this in the model. However, it is difficult to take swelling of the hydrogel into account due to the subsequent interface displacement. The fourth difficulty is that due to surface tension and adhesive forces the interface between both phases is not flat, 
instead a curved meniscus emerges. This meniscus makes it difficult to define the correct axial position for the interface in the model. A priori simulations show that the estimates for the diffusion coefficients strongly depend on the assumed position of the interface. A displacement of the interface by $1 \mathrm{~mm}$ in the kinetic model causes a change of the estimated diffusion coefficient in the gel by $8.6 \%$ (results not shown). Moreover, close to the meniscus radial concentration gradients are formed which cannot be measured by NMR, which averages over the whole plane. Therefore, measurements close to the interface are erroneous. On the other hand, if measurements are conducted further away from the interface, it would last longer until the substance reaches the measurement zone. This would deteriorate the problems of evaporation and swelling.

Since the presented estimation of the diffusion coefficients is based on one single experiment, further experiments are necessary in order to overcome the experimental difficulties and to get a more reliable estimate for the diffusion coefficients. This could be achieved by performing several MEXA cycles in combination with the incremental approach for the identification of diffusive transport proposed by Bardow and Marquardt (2004a).

\subsubsection{Conclusions}

Spectroscopic non-invasive measurement techniques are the most suitable methods for the determination of diffusion coefficients in hydrogels. An alternative to the previously applied CLSM is NMR. For this reason, it was investigated if NMR is also suitable for the determination of the diffusion coefficient of DMBA in K-carrageenan hydrogel. In contrast to CLSM, NMR requires hydrogel cylinders, since only axial profiles can be measured. An axial diffusion model was developed and fitted to the data of one initial experiment. Due to several experimental challenges like gel swelling and formation of a meniscus at the interface the obtained diffusion coefficient for DMBA in $\kappa$-carrageenan hydrogel differs significantly from the result obtained with CLSM. Although further experiments would probably improve the estimation procedure, CLSM is recommended for the determination of diffusion coefficients of fluorescent substances, due to its higher spatial and temporal resolution. However, NMR has the advantage that it can be applied to every kind of organic substance. 


\section{Coupling of Enzyme and Mass Transfer Kinetics}

A drawback for the biocatalytic production of hydrophobic fine chemicals is the low solubility of reactants in aqueous media. To overcome this, the addition of a water-immiscible organic solvent may be beneficial. The organic phase acts both as substrate reservoir and as in situ extraction solvent (Halling, 1994). Hence, higher yields and productivities can be achieved and inhibitions or undesired side-reactions suppressed (Diender et al., 2002; Eckstein et al., 2006a; Fernandes and Cabral, 2008; Gröger et al., 2004; Peters et al., 2007; Rosche et al., 2004; Villela Filho et al., 2003). In such aqueous-organic biphasic reaction systems a superposition of reaction and mass transfer takes place. This makes process design more complex (Halling, 1994) and may result in mass transfer limitation which lowers the productivity (Baldascini et al., 2001). However, in certain cases mass transfer limitation can also be favorable, for example in order to suppress a chemical side reaction (Gerrits et al., 2001; Willeman et al., 2002a). Mass transfer limitation can be avoided by decreasing the enzyme concentration or by increasing agitation or interfacial area (Straathof, 2003). In this chapter, two kinds of biphasic systems are studied. In the first Section the biphasic system consists of two distinct phases with a defined interfacial area. In the second Section an industrially more relevant emulsified system with an increased interfacial area is investigated. Moreover, the problems for characterizing an emulsified system are discussed.

\subsection{Biphasic reactive system with two distinct phases}

\subsubsection{Introduction}

The studied reaction is the stereoselective carboligation of two 3,5-dimethoxy-benzaldehyde (DMBA) molecules to $(R)-3,3$ ',5,5'-tetramethoxy-benzoin (TMB) using benzaldehyde lyase (BAL, EC 4.1.2.38) from Pseudomonas fluorescens. The kinetics of this enzyme were investigated in Chapter 2. It was demonstrated that in an aqueous phase only low final product concentrations can be achieved. In order to maximize the extractable yield, a systematic approach was applied for solvent selection (Chapter 3). This approach revealed that methyliso-butyl-ketone (MIBK) is a suitable solvent. The mass transfer and partition coefficients were quantified. The aim of this Section is to investigate the effects of coupling both 
phenomena, enzyme kinetics and mass transfer, in an aqueous-organic biphasic reaction system. Therefore, the kinetics for both individual phenomena are combined in one model. According to the model-based experimental analysis (MEXA) approach the model is used to perform a priori simulations and a sensitivity analysis in order to choose a suitable measurement method. Subsequently, the model is used to design optimal experiments for parameter estimation (Marquardt, 2005).

\subsubsection{Model development}

The kinetic models for the enzyme reaction (Chapter 2.2) and for the mass transfer (Chapter 3) were coupled to one kinetic model. Eqs. 5-1 and 5-2 are the mass balances for the organic and the aqueous phase, where $c_{i}$ denotes the concentration of the compound $i . V_{\text {org }}$ and $V_{a q}$ are the volumes of organic and aqueous phase, $\dot{n}_{i, M T}$ is the mass transfer flux, $\dot{n}_{i, R}$ the reaction flux.

$$
\begin{gathered}
\frac{d c_{i, \text { org }}}{d t} \cdot V_{\text {org }}=\dot{n}_{i, M T} \\
\frac{d c_{i, a q}}{d t} \cdot V_{a q}=-\dot{n}_{i, M T}+\dot{n}_{i, R}
\end{gathered}
$$

The mass transfer flux is described by Eq. 5-3, where $k_{i}$ is the mass transfer coefficient and $P_{i}$ the partition coefficient of compound $i$.

$$
\dot{n}_{i, M T}=k_{i} \cdot A \cdot\left(P_{i} \cdot c_{i, a q}(t)-c_{i, o r g}(t)\right)
$$

The reaction flux is calculated using the stoichiometric coefficient $v_{i}$ and the reaction rate $v$.

$$
\dot{n}_{i, R}=v_{i} \cdot v \cdot V_{a q}
$$

Eqs. 5-5 and 5-6 constitute the mechanistic kinetic model for symmetric carboligations using BAL, which was derived in Chapter 2.2. $k_{c a t}, K_{e q}, K_{m A}, K_{m B}, K_{m P}, K_{i A}$, and $K_{i B}$ are the enzyme kinetic parameters. 


$$
\begin{gathered}
v=\frac{\frac{K_{c a t f}}{K_{i A} \cdot K_{m B}} \cdot\left(c_{A, a q}^{2}-\frac{c_{P, a q}}{K_{e q}}\right)}{1+\frac{c_{A, a q}}{K_{i A}}\left(1+\frac{K_{m A}}{K_{m B}}\right)+\frac{c_{A, a q}{ }^{2}}{K_{i A} \cdot K_{m B}}+\frac{c_{P}}{K_{m P}}+\frac{c_{A} \cdot c_{P}}{K_{m P} \cdot K_{i B}}} \cdot c_{E} \\
K_{i B}=\frac{K_{m B} \cdot K_{i A}}{K_{m A} \cdot\left[1-\left(\frac{K_{m A}}{K_{i A}}-1\right) \cdot \frac{K_{m P}}{K_{e q} \cdot K_{m B} \cdot K_{i A}}\right]}
\end{gathered}
$$

As demonstrated in Chapter 2.2, the enzyme kinetic parameters are not identifiable unless a simplification is introduced. Thus, the model contains two additional equations:

$$
\begin{gathered}
K_{m P}=\frac{K_{m B} \cdot\left(K_{m B}-K_{m A}\right)^{2} \cdot K_{e q}}{2 \cdot K_{m A}} \\
K_{i A}=K_{m B}-K_{m A}
\end{gathered}
$$

\subsubsection{A priori simulations}

According to the MEXA approach the kinetic model is used for a priori simulations which support the identification of a suitable measurement method. In this case, the question which substance should be quantified in order to determine the unknown model parameters has to be answered. Therefore, four in silico experiments are conducted (Table 5-1). For this purpose, the model is used to simulate concentration profiles which are subsequently disturbed using Gaussian noise with a constant standard deviation of $0.05 \mathrm{mM}$. The nominal values (Table 5-2) used for the simulation are taken from Chapters 2 and 3.

Four test cases are investigated: In the first case it is assumed that only the substrate concentration in the aqueous phase $c_{A, a q}$ can be measured. In the second case also $c_{P, a q}$ is considered to be quantifiable. In the third case $c_{A, \text { org }}$ and in the fourth case $c_{P, \text { org }}$ are additionally taken into account. For all four test cases a parameter estimation is performed. 
Table 5-1: $\quad$ Overview over in silico experiments. The enzyme concentration $c_{E}$ is in each case $1.67 \cdot 10^{-5} \mathrm{mM}$ and the experiment duration $t$ is $10,000 \mathrm{~s}$.

\begin{tabular}{lllll}
\hline Experiment & $c_{A, \text { aq }}[\mathrm{mM}]$ & $c_{P, \text { aq }}[\mathrm{mM}]$ & $c_{A, \text { org }}[\mathrm{mM}]$ & $c_{P, \text { org }}[\mathrm{mM}]$ \\
\hline A & 0 & 0 & 25 & 0 \\
B & 0 & 0 & 0 & 25 \\
C & 5 & 0 & 0 & 0 \\
D & 0 & 2.5 & 0 & 0 \\
\hline
\end{tabular}

Table 5-2: $\quad$ Nominal values for the model parameters (determined in Chapters 2 and 3).

\begin{tabular}{lll}
\hline Parameter & Unit & Nominal value \\
\hline$k_{A}$ & $\mathrm{~m} \mathrm{~s}^{-1}$ & $5.39 \cdot 10^{-7}$ \\
$k_{P}$ & $\mathrm{~m} \mathrm{~s}^{-1}$ & $2.81 \cdot 10^{-7}$ \\
$P_{A}$ & - & 52.37 \\
$P_{P}$ & - & 84.36 \\
$k_{\text {catf }}$ & $\mathrm{s}^{-1}$ & 40.37 \\
$K_{\text {eq }}$ & $\mathrm{mM}^{-1}$ & 1.464 \\
$K_{m A}$ & $\mathrm{mM}$ & 0.147 \\
$K_{m B}$ & $\mathrm{mM}$ & 0.269 \\
\hline
\end{tabular}

Figure 5-1 shows the obtained 95\% confidence ellipsoids for the mass transfer coefficient $k_{A}$ and the maximum turnover number $k_{\text {catf. }}$ Obviously, the confidence region strongly decreases, if more concentrations can be measured. However, no difference between case 3 and 4 can be seen. The fourth concentration does not provide further information as it can be calculated from the three other concentrations using a mass balance. Moreover, the confidence ellipsoid indicates a strong correlation of both parameters. The absolute values of the correlations decrease for the four cases $(-0.92,-0.71,0.45$, and 0.45$)$. A decrease of confidence regions and correlations can be also observed for the other parameters (results not shown). 


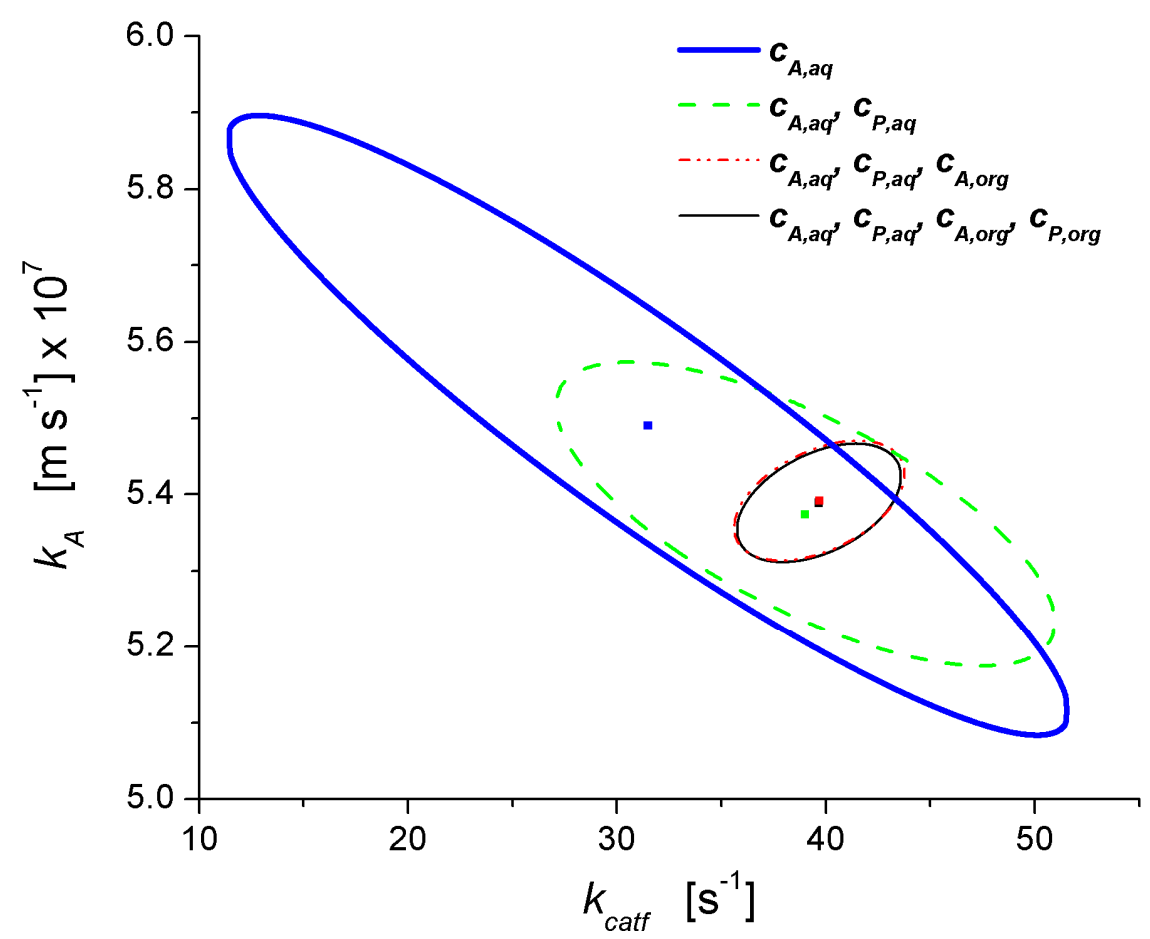

Figure 5-1: $\quad 95 \%$ confidence ellipsoids for $k_{A} / k_{\text {catf }}$ for the four investigated test cases. Case 1: only $c_{A, a q}$ quantifiable; case 2: $c_{A, a q}$ and $c_{P, a q}$ quantifiable; case 3: $c_{A, a q}, c_{P, a q}$, and $c_{A, \text { org }}$ quantifiable; case 4: all concentrations are quantifiable.

From these findings it can be concluded that the parameters are not identifiable, if measurements are carried out in the aqueous phase only. The explanation for this behavior is that the aqueous phase is the reactive phase. Thus, concentration changes depend both on mass transfer and reaction rate (Eq. 5-2). For example, a decrease of $c_{A, a q}$ can be caused by either the reaction to the product or by a mass transfer to the organic phase. This is the reason for the strong correlation of $k_{A}$ and $k_{\text {catf }}$ in case 1 . In contrast to this, concentration changes in the organic phase are caused by mass transfer only. Thus, measurements in the organic phase allow the quantification of the mass transfer of the respective reactant. This minimizes the correlation of mass transfer parameters like $k_{A}$ and enzyme kinetic parameters like $k_{\text {catf. }}$. In conclusion, measurements in both phases are required and permit the determination of intrinsic reaction kinetics without the masking effects of mass transfer (Marquardt, 2005; Michalik et al., 2009). However, this requirement is often ignored and sometimes even denied. For example, Straathof (2003) argues that measurements in the aqueous phase may easily be below the detection limit and are of little use for drawing any conclusions. 


\subsubsection{Sensitivity analysis}

Using the nominal parameter values listed in Table 5-2 a sensitivity analysis is carried out. The sensitivities $S_{i, j}$ are calculated by automatic differentiation using ADiMat (Vehreschild, 2004) and normalized using Eq. 5-9, where $\theta_{j}$ is the parameter $j$ and $c_{i}$ the concentration of compound $i$.

$$
S_{i, j}=\frac{\theta_{j}}{c_{i}} \cdot \frac{\partial c_{i}}{\partial \theta_{j}}
$$

Figure 5-2 shows the normalized sensitivities of the model parameters with respect to $c_{A, \text { org }}$. It becomes obvious that the system is mass transfer limited since the enzyme kinetic parameters $k_{c a t f}, K_{m A}$, and $K_{m B}$ are not sensitive at all. Therefore, these parameters are not identifiable in a parameter estimation based on experimental data obtained under the specified conditions. In contrast to this, the mass transfer coefficients are sensitive in the beginning. The equilibrium is determined by the thermodynamic equilibrium constant $K_{e q}$, and by the partition coefficients $P_{A}$ and $P_{P}$. In equilibrium the mass transfer and reaction flux become zero. Based on Eqs. 5-3 and 5-5 the equilibrium concentration of $c_{A, \text { org }}$ can be calculated as shown in Eq. 5-10. Since $K_{e q}$ and $P_{P}$ appear only as product, their influences on the equilibrium concentration are the same which explains why their sensitivity curves in Figure 5-2 are identical.

$$
c_{A, \text { org }}(t \rightarrow \infty)=P_{A} \cdot \sqrt{\frac{c_{P, \text { org }}(t \rightarrow \infty)}{P_{P} \cdot K_{\text {eq }}}}
$$

Figure 5-3 shows the normalized sensitivities with respect to $c_{A \text {, org }}$ for a reaction limited system. In contrast to the mass transfer limited system, the mass transfer coefficients are no longer sensitive. For this reason, they are not identifiable in a reaction limited system. In contrast to this, the normalized sensitivities of the enzyme kinetic parameters significantly differ from zero. For this reason, experiments should be conducted in the reaction limited regime in order to determine the enzyme kinetic parameters. 


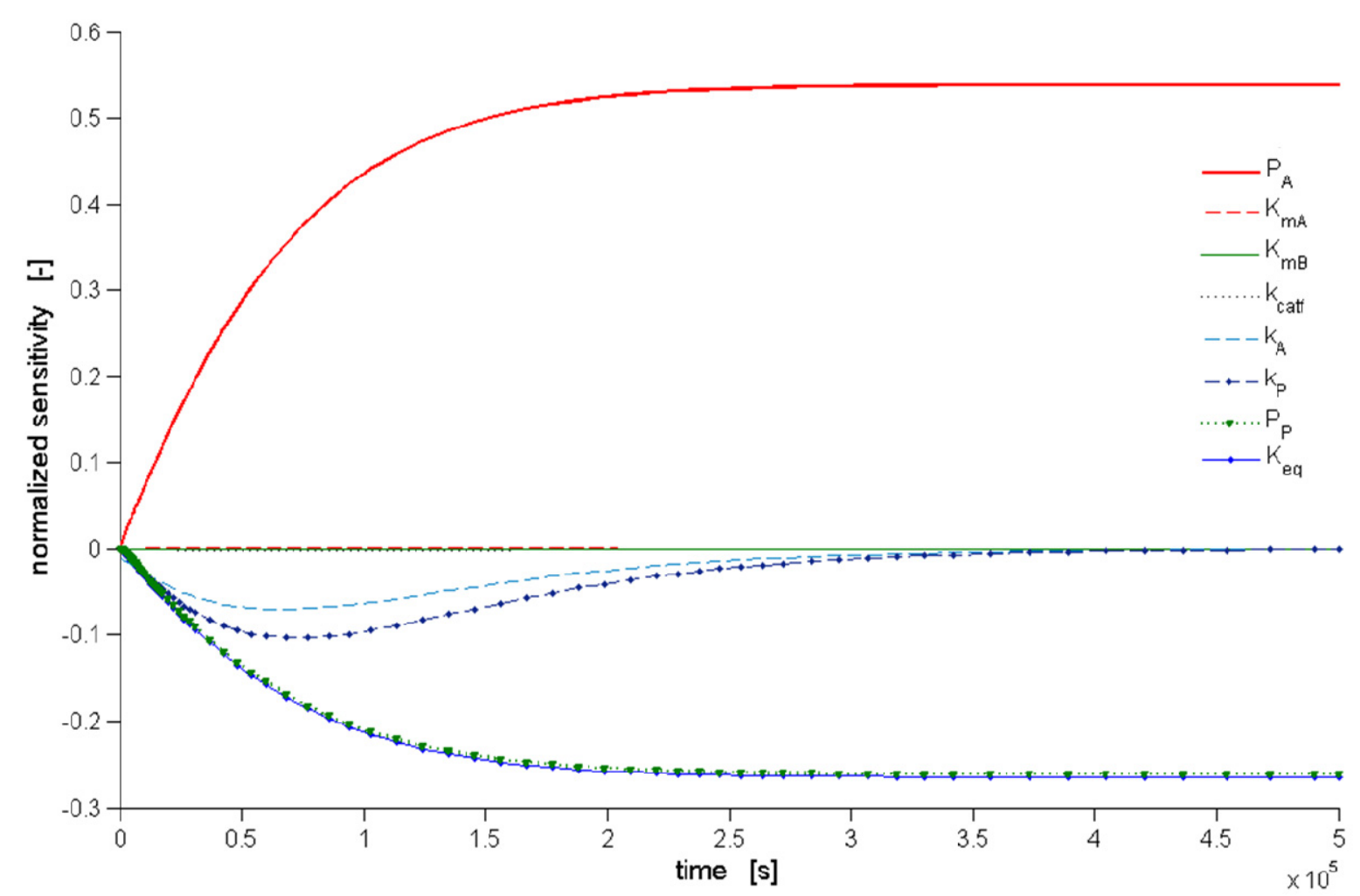

Figure 5-2: Normalized sensitivities of the model parameters regarding $c_{A, \text { org }}$. The initial conditions were $c_{A, \text { org }}=10 \mathrm{mM}, c_{A, a q}=0 \mathrm{mM}, c_{P, a q}=0 \mathrm{mM}$, and $c_{P, o r g}=0 \mathrm{mM}$. The enzyme concentration was $c_{E}=3.34 \cdot 10^{-4} \mathrm{mM}$. The sensitivities were calculated via automatic differentiation.

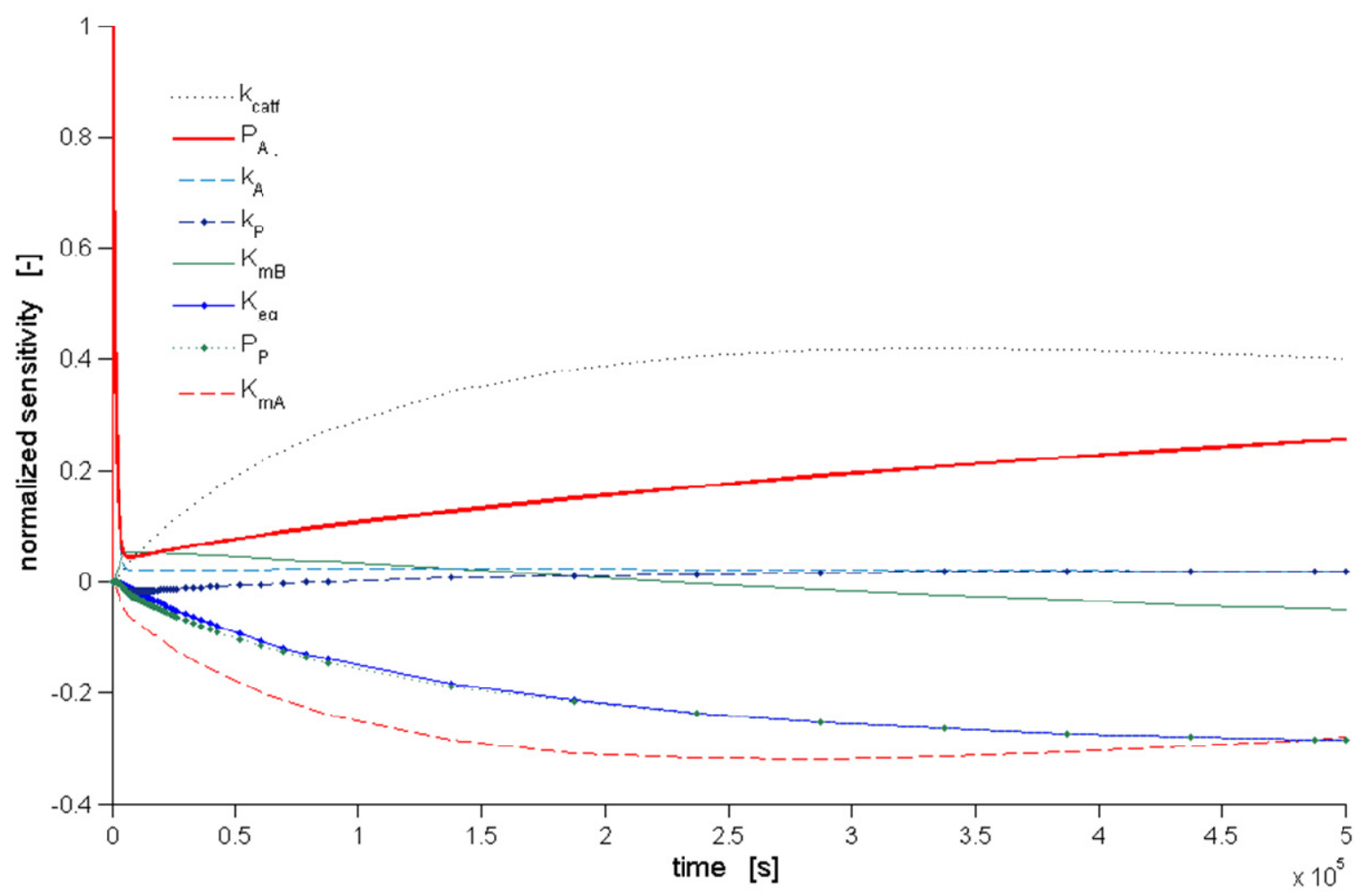

Figure 5-3: Normalized sensitivities of the model parameters regarding $c_{A, \text { org }}$. The initial conditions were $c_{A, \text { org }}=0 \mathrm{mM}, c_{A, a q}=0.96 \mathrm{mM}, c_{P, a q}=2.5 \mathrm{mM}$, and $c_{P, \text { org }}=0 \mathrm{mM}$. The enzyme concentration was $c_{E}=3.38 \cdot 10^{-7} \mathrm{mM}$. The sensitivities were calculated via automatic differentiation. 
Mass transfer limitation does not only hamper the estimation of the enzyme kinetic parameters, but may lead to a phenomenon which is not considered in the kinetic model (Eqs. 5-1-8). The kinetic model assumes that the enzyme reaction takes place in the aqueous bulk phase, whereas in the boundary layer only mass transfer occurs. However, this is only true if the reaction can be considered to be much slower than mass transfer. If this is not the case, a part of the reaction occurs within the boundary layer. Therefore, the concentration gradient is increased and the value of the mass transfer coefficient appears to be higher (Figure 5-4).
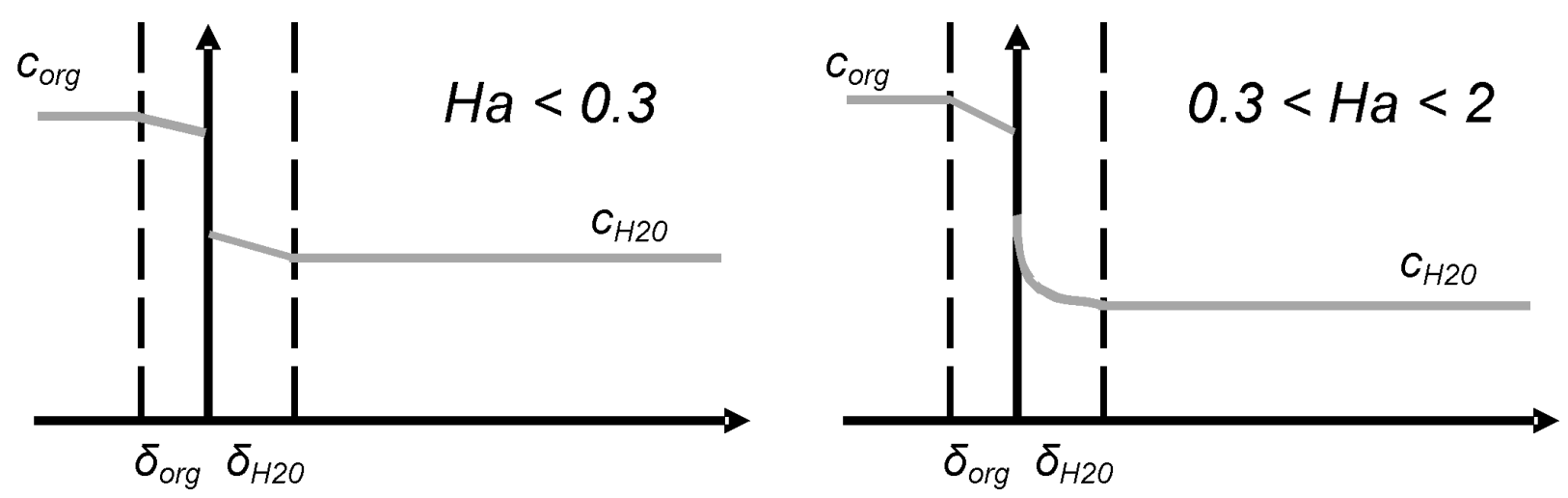

Figure 5-4: Illustration of the concentration profiles at the interface for a reaction limited regime (left) and for a (partially) mass transfer limited regime (right). $\delta_{\mathrm{i}}$ denotes the film thickness.

The dimensionless Hatta number $H a$ relates the reaction flux to the mass transfer flux (Eq. 511). Three regimes can be classified: If $H a<0.3$, the reaction rate is much slower than the mass transfer rate and the system is reaction limited. In the boundary layer only mass transfer takes place (Figure 5-4, left) In contrast to this, $H a>2$ indicates a mass transfer limitation. In this case, the reaction completely takes place in the boundary layer. If $0.3<\mathrm{Ha}<2$, the system is in the intermediate regime, where a part of the reaction occurs in the boundary layer (Figure 5-4, right). To consider reaction within in the boundary layer, a spatially distributed model would be necessary. However, no measurements in the boundary layer are possible and the identification of the model parameters would be unnecessarily complex (Michalik et al., 2009). For this reason, the reaction should be carried out in the reaction limited regime and thus $H a<0.3$ is considered as a constraint for the optimal experimental design (OED).

$$
H a_{i}=\frac{\dot{n}_{i, R}}{-\dot{n}_{i, M T}}
$$


Despite these considerations, it is not certain if the Hatta-concept is applicable for enzyme reactions since the concept was initially developed for chemical catalysts. Due to the much larger size of enzymes (the BAL tetramer has a size of approximately $9500 \mathrm{pm} \cdot 9500 \mathrm{pm}$. 7500 pm (Mosbacher et al., 2005)) compared to chemical catalysts (e.g. the heavy metal ion $\mathrm{Pt}^{2+}$ has an ion radius of $80 \mathrm{pm}$ ) it is not clear whether the enzymes are not too large to penetrate into the boundary layer with their active sites. Only few publications can be found where the Hatta-concept was utilized for enzyme reactions (Fernandes and Cabral, 2008; Trusek-Holownia, 2003; Trusek-Holownia and Noworyta, 2000). In these publications only a first-order reaction constant was used for the calculation of the Hatta-number and it was ignored that the reaction rate is not constant, but depends on the concentrations of the reactants. However, even if the Hatta-concept cannot be applied to enzyme reactions, the constraint $H a<0.3$ will guarantee that the process is carried out in the reaction limited regime in which the enzyme reaction parameters are sensitive (Figure 5-3) and thus can be identified.

\subsubsection{Materials and methods}

As the a priori simulations revealed, measurements in both phases have to be conducted. On this basis a new reactor concept was developed, which is illustrated in Figure 5-5. The reactor is similar to a Lewis cell (Fernandes and Cabral, 2008) where both phases are stirred independently ( $240 \mathrm{rpm}$ for the upper phase, $264 \mathrm{rpm}$ for the lower phase). Each phase is considered to be ideally mixed. The size of the interface remains constant $\left(3.14 \mathrm{~cm}^{2}\right)$. From both the organic and aqueous phase medium is pumped through tubes into an absorption spectrophotometer (SpectraMax Plus, Molecular Devices, Sunnywale, USA). Medium from the aqueous phase is additionally pumped into a fluorescence spectrophotometer (LS55, Perkin Elmer, Waltheim, USA). Thus, the extinctions in the organic and aqueous phase and the fluorescence in the aqueous phase can be detected. The pumping rate of the membrane pumps was chosen sufficiently high so that circulation time can be neglected in the kinetic model (Schmidt, 2008).

According to the solvent selection approach described in Chapter 3, MIBK was chosen as

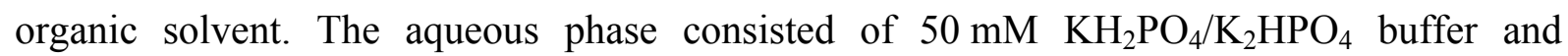
25\%(v/v) dimethylformamide (DMF). The cosolvent DMF was used to increase the solubility of the aromatic compounds. The $\mathrm{pH}$ value was adjusted to 8.5 and the ionic strength to $100 \mathrm{mM}$. The concentration of the cofactors $\mathrm{MgSO}_{4}$ and thiamin diphosphate (ThDP) was 
$0.25 \mathrm{mM}$. The temperature was kept constant at $25^{\circ} \mathrm{C}$. All reagents were of analytical grade and obtained from Sigma-Aldrich (Deisenhofen, Germany).

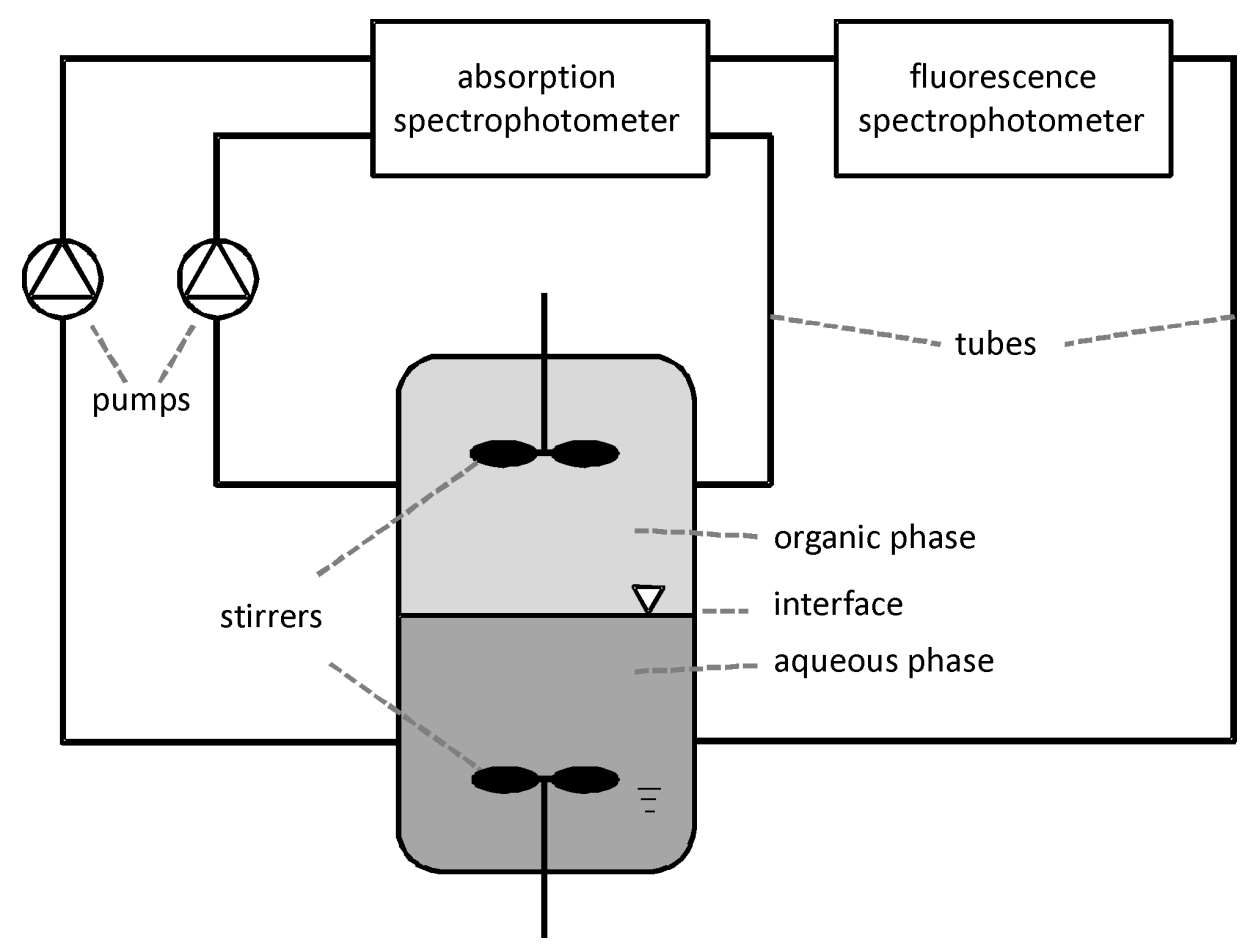

Figure 5-5: Newly developed reactor concept which allows measurements in both phases

The extinction in the organic and in the aqueous phase was measured at $325 \mathrm{~nm}$. At this wavelength both reactants absorb light. The resulting extinction Ext can be described by the Lambert-Beer law (Eq. 5-12). The extinction coefficients $\varepsilon_{i}$ were determined to $2.796 \mathrm{mM}^{-}$ ${ }^{1} \mathrm{~cm}^{-1}$ and $2.540 \mathrm{mM}^{-1} \mathrm{~cm}^{-1}$ for DMBA and TMB in the organic phase, respectively. In the aqueous phase they were $2.434 \mathrm{mM}^{-1} \mathrm{~cm}^{-1}$ and $2.260 \mathrm{mM}^{-1} \mathrm{~cm}^{-1}$. The pathlength $d$ of the cuvette was $0.5 \mathrm{~mm}$. The background extinction $b$ was determined for each experiment individually.

$$
E x t=\left(\varepsilon_{A} \cdot c_{A}+\varepsilon_{P} \cdot c_{P}\right) \cdot d+b
$$

Fluorescence measurements were conducted in the aqueous phase using a wavelength of $360 \mathrm{~nm}$ for excitation and of $470 \mathrm{~nm}$ for emission. As discussed in Chapter 2.5.3.1 the fluorescence intensity $I$ depends on both the DMBA and TMB concentration:

$$
I=\left(\left(a \cdot c_{A}^{3}+b \cdot c_{A}^{2}+c \cdot c_{A}\right) \cdot\left(1-d \cdot c_{P}\right)+e \cdot c_{P}^{3}+f \cdot c_{P}^{2}+g \cdot c_{P}\right) \cdot q+s
$$


The calibration parameters slightly differ from those obtained in the monophasic aqueous system, probably due to the partial solubility of MIBK in the aqueous phase. The parameters $a-g$ are listed in Table 5-3. The relative error between calibration model and experimental data is $4.1 \%$. Fluctuations of the fluorescence intensity from day to day were considered using the parameter $q$. The background fluorescence $s$ was determined prior to addition of the reactants. In the organic phase no fluorescence could be detected, as MIBK quenches the fluorescence.

Table 5-3: Calibration parameter for the fluorescence measurements in the aqueous phase of the biphasic system (excitation wavelength: $360 \mathrm{~nm}$; emission wavelength: $470 \mathrm{~nm}$ ).

\begin{tabular}{llc}
\hline Parameter & Unit & Estimate \\
\hline$a$ & $\mathrm{mM}^{-3}$ & 0.19 \\
$b$ & $\mathrm{mM}^{-2}$ & -5.90 \\
$c$ & $\mathrm{mM}^{-1}$ & 69.28 \\
$d$ & $\mathrm{mM}^{-1}$ & 0.05 \\
$e$ & $\mathrm{mM}^{-3}$ & 0.01 \\
$f$ & $\mathrm{mM}^{-2}$ & -0.23 \\
$g$ & $\mathrm{mM}^{-1}$ & 2.72 \\
\hline
\end{tabular}

The calibration models (Eqs. 5-12 and 5-13) with fixed calibration parameters were integrated into the kinetic model. Thus, the model could be directly fitted to the experimental extinction and fluorescence intensities. Alternatives to this approach are discussed in Chapter 2.5.

\subsubsection{Results and discussion}

Based on the nominal values of Table 5-2, optimal experiments were designed using the E-criterion which aims at minimizing the largest eigenvalue of the variance-covariance matrix (Walter and Pronzato, 1990). In order to stay in the reaction limited regime, the optimized enzyme concentration was on average two orders of magnitude lower compared to the enzymatic experiments described in Chapter 2. Table 5-4 lists the optimized conditions of these experiments. 
Table 5-4: Optimized experiments for the aqueous-organic biphasic reaction system. The degrees of freedom were $c_{A, a q}$ (limits: 0-5 mM), $c_{A, \text { org }}$ (limits: 0-25 mM), $c_{P, a q}$ (limits: 0-2.5 $\left.\mathrm{mM}\right), c_{P, \text { org }}$ (limits: $0-10 \mathrm{mM}$ ), $c_{E}$ (limits: $10^{-8}-10^{-5} \mathrm{mM}$ ), and $t$ (limits: $600-14,400 \mathrm{~s}$ ). Additional constraints were $c_{A, \text { org }}+c_{P, \text { org }}<25 \mathrm{mM}$ and $\mathrm{Ha}<0.3$.

\begin{tabular}{lccllll}
\hline Experiment & $c_{A, \text { aq }}[\mathrm{mM}]$ & $c_{A, \text { org }}[\mathrm{mM}]$ & $c_{P, \text { aq }}[\mathrm{mM}]$ & $c_{P, \text { org }}[\mathrm{mM}]$ & $c_{E}[\mathrm{mM}]$ & $t[\mathrm{~s}]$ \\
\hline $\mathrm{E}$ & 0.89 & 2.51 & 0.11 & 9.94 & $9.81 \cdot 10^{-8}$ & 622 \\
$\mathrm{~F}$ & 2.79 & 0.15 & 1.22 & 0.00 & $9.79 \cdot 10^{-7}$ & 4296 \\
$\mathrm{G}$ & 2.09 & 16.71 & 0.80 & 0.00 & $7.32 \cdot 10^{-7}$ & 3292 \\
$\mathrm{H}$ & 0.41 & 0.00 & 1.45 & 0.00 & $1.44 \cdot 10^{-7}$ & 3391 \\
$\mathrm{I}$ & 5.00 & 25.00 & 1.18 & 0.00 & $1.08 \cdot 10^{-6}$ & 8015 \\
$\mathrm{~J}$ & 0.96 & 0.00 & 2.50 & 0.00 & $3.38 \cdot 10^{-7}$ & 12532 \\
\hline
\end{tabular}

A sequential approach was followed for the parameter estimation. In a first step, the unknown fluorescence factor $q$ was individually estimated for each experiment. Thus, fluctuations of the fluorescence intensity from experiment to experiment were taken into account. The fit of the kinetic model to the experimental data is shown in Figure 5-6.

The kinetic model fits the experimental data satisfactorily (Figure 5-6). The obtained parameter estimates are listed in Table 5-5. The partition coefficients are determined precisely and deviate only slightly from the nominal values determined in Chapter 3 . The estimates for the enzyme kinetic parameters also differ only marginally from the nominal values obtained in Chapter 2. However, their standard deviations are unacceptably large. Moreover, all enzyme kinetic parameters are highly correlated (Table 5-6). Thus, they are not identifiable under the applied conditions and no statistically confirmed conclusions can be drawn regarding the question if their values are influenced by the occurrence of an organic phase. 

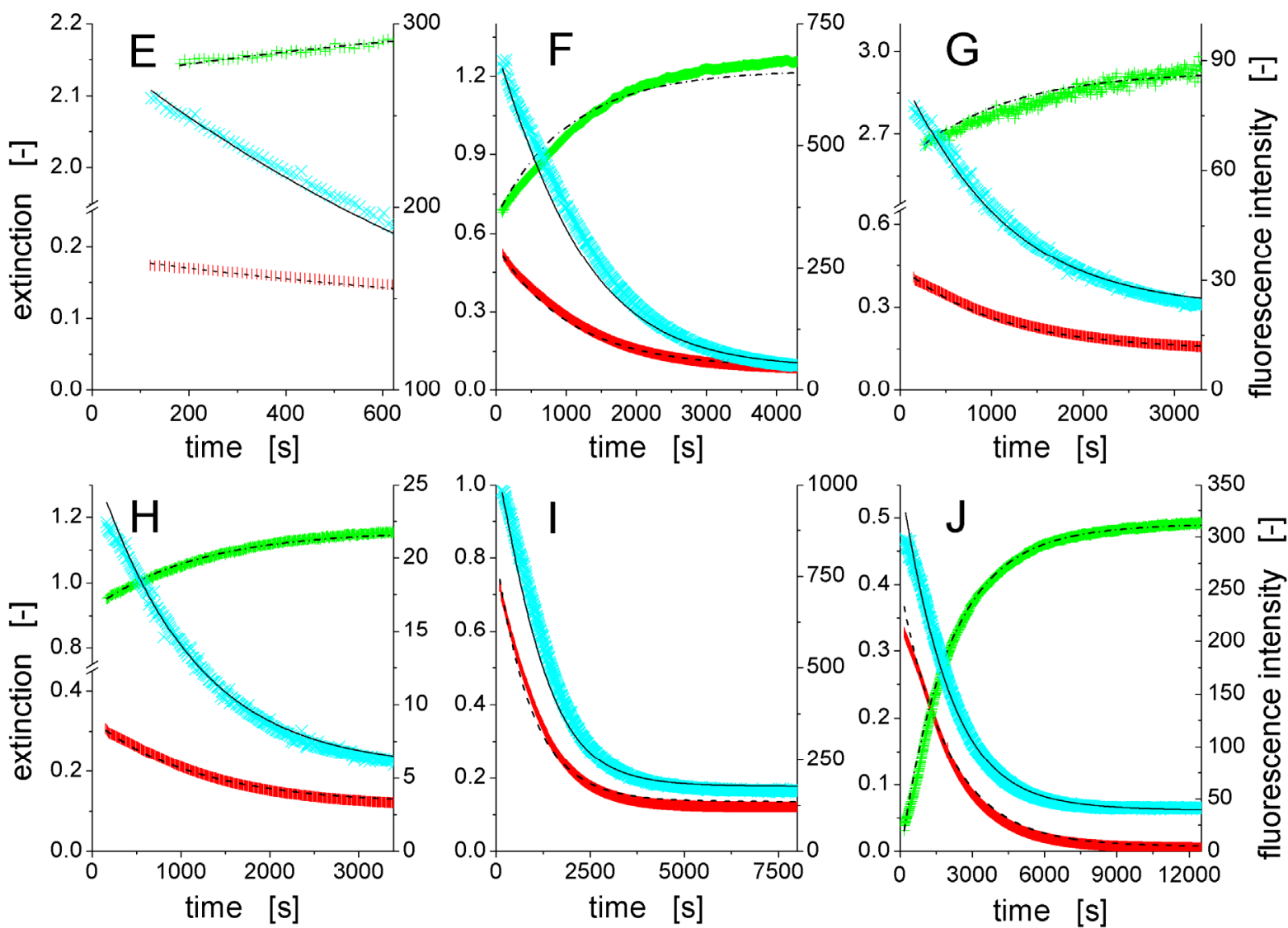

$\mid \mathrm{Ext}_{\mathrm{aq}}(\exp .) \cdots-\mathrm{Ext}_{\mathrm{aq}}($ model $)+\mathrm{Ext}_{\mathrm{org}}(\exp .) \cdots-\mathrm{Ext}_{\mathrm{org}}$ (model) $\times \mathrm{I}$ (exp.) $-\mathrm{I}$ (model)

Figure 5-6: $\quad$ Fit of the kinetic model to experimental data from extinction and fluorescence measurements. The experimental conditions are listed in Table 5-4. (In experiment $\mathrm{E}$ the first $120 \mathrm{~s}$ were omitted due to large noise. In experiment I the extinction measurements in the organic phase were defective and could not be used.)

Table 5-5: Comparison of the nominal parameter values with the estimates using the biphasic reactive system. The mass transfer coefficients were not identifiable due to reaction limitation.

\begin{tabular}{llllc}
\hline Parameter & Unit & Nominal value & Estimate & Standard deviation \\
\hline$P_{A}$ & - & 52.37 & 52.76 & 0.17 \\
$P_{P}$ & - & 84.36 & 82.25 & 0.30 \\
$k_{\text {catf }}$ & $\mathrm{s}^{-1}$ & 40.37 & 42.51 & 18.17 \\
$K_{e q}$ & $\mathrm{mM}^{-1}$ & 1.464 & 1.577 & 0.554 \\
$K_{m A}$ & $\mathrm{mM}$ & 0.147 & 0.136 & 0.109 \\
$K_{m B}$ & $\mathrm{mM}$ & 0.269 & 0.251 & 0.141 \\
\hline
\end{tabular}


Table 5-6: $\quad$ Correlation matrix of the estimated parameters using the biphasic reactive system.

\begin{tabular}{lllllll}
\hline & $P_{A}$ & $P_{P}$ & $K_{\text {catf }}$ & $K_{e q}$ & $K_{m A}$ & $K_{m B}$ \\
\hline$P_{A}$ & 1.00 & -0.52 & 0.26 & -0.63 & 0.29 & 0.29 \\
$P_{P}$ & & 1.00 & -0.13 & 0.30 & -0.16 & -0.17 \\
$k_{\text {catf }}$ & & & 1.00 & -0.86 & 0.97 & 0.94 \\
$K_{e q}$ & & & & 1.00 & -0.86 & -0.83 \\
$K_{m A}$ & & & & & 1.00 & 0.99 \\
$K_{m B}$ & & & & & & 1.00 \\
\hline
\end{tabular}

To increase the precision of the kinetic parameters more optimized experiments should be conducted. However, the reproducibility of the fluorescence measurements has been poor so far caused by numerous possible factors. Among them are a carry-over of fluorophores, intensity fluctuations of the light source, and a change of the angle between light beam and cuvette glass due to a torsion in the cuvette holder caused by the tubes. Furthermore, bleaching due to a too long exposure to light, and quenching caused by small amounts of impurities are possible. In order to improve the reliability, an additional high performance liquid chromatography (HPLC) measurement at the end of each experiment could be helpful. Using HPLC the final concentrations of each substance could be quantified without introducing a bias due to sampling. Thus, the validity of the calibration parameters could be checked at the end of each experiment and additional information for parameter estimation would be gained.

One further problem using the biphasic reactive system is that the optimized enzyme concentrations are extremely low. Thus, additional dilution steps have to be performed which increase the overall pipetting error. Another issue, which should be investigated in more detail, is the possibility of enzyme deactivation in the biphasic system. Enzyme deactivation can occur due to molecular toxicity and due to phase toxicity (Halling, 1987). Molecular toxicity is caused by organic solvents which show a partial solubility in water. Individual organic solvent molecules might interact with the enzymes. These interactions can be the direct binding to the active sites of the enzymes or the alteration of the micro-environment of the enzymes resulting in a destabilization or in replacing water molecules required for the catalytic mechanism (Butler, 1979; Fernandes and Cabral, 2008; Halling, 1994). According to the $\log \mathrm{P}$-concept more hydrophobic solvents are less deactivating due to their lower solubility 
in the aqueous phase (Laane et al., 1987). However, Villela Filho et al. (2003) demonstrate that the chemical functionality of the solvent molecules can be more decisive than the $\log \mathrm{P}$ value. Phase toxicity does not follow the logP-concept (Halling, 1994). According to Baldascini and Janssen (2005) enzymes can adsorb at the interface when they come in contact with it. Then the hydrophobic segments may become exposed which leads to unfolding, aggregation, and precipitation.

Using classical storage stability tests in the presence of MIBK no significant molecular toxicity could be observed for BAL (Schmidt, 2008). Phase toxicity due to MIBK cannot be excluded. However, the interfacial area is quite small for the used setup and no enzyme precipitation can be observed. Moreover, the mechanical stress caused by pumping might decrease enzyme stability (Schmidt, 2008). The integration of a first-order enzyme deactivation term into the kinetic model has been tested, but has no positive effect. The deactivation constant cannot be estimated sufficiently precise and the standard deviations of all other parameters increase further (results not shown).

\subsubsection{Conclusions}

To investigate the kinetics of BAL within an aqueous-organic biphasic reaction system a kinetic model was derived which considers mass transfer and enzyme reaction. Using a priori simulations it could be demonstrated that measurements in both phases are required to determine the kinetic parameters. On the basis of these results a new reactor concept was developed which allows extinction measurements in both phases and fluorescence measurements in the aqueous phase. A sensitivity analysis demonstrated that experiments have to be carried out in the reaction limited regime in order to identify the enzyme kinetic parameters. The kinetic model was able to describe the data from optimized experiments satisfactorily. The partition coefficients could be estimated precisely and are in accordance with previous results of mass transfer experiments without enzyme present (Chapter 3). In contrast to this, the enzyme kinetic parameters were not identifiable under the applied conditions. Not only more optimized experiments are required, but also improvements of the experimental techniques in terms of precision and reliability are necessary. Moreover, a possible enzyme deactivation should be further investigated. 


\subsection{Reactive emulsion}

\subsubsection{Introduction}

As demonstrated in the previous Section the enzyme kinetic parameters can only be identified if the experiment is carried out in the reaction limited regime. This can be achieved by either decreasing the reaction rate or accelerating the mass transfer. The first possibility can be realized by reducing the enzyme concentration as shown in the previous Section. The other possibility is to enhance mass transfer by increasing agitation. This results in a decreased boundary layer and can enlarge the mass transfer area. At high power inputs the biphasic system forms an emulsion (Straathof, 2003).

Since only the second possibility leads to high volumetric productivities, emulsified systems are often used in industry. Emulsions serve not only as reaction systems for enzymatic conversions involving hydrophobic reactants, but also as vehicle for drug delivery (Klyachko and Levashov, 2003; Lawrence and Rees, 2000; Rairkar et al., 2007). However, until today no possibility exists for studying emulsions in small scale using quasi-continuous measurement techniques. So far, the standard procedure is to emulsify the biphasic system for only a certain period of time. Then the stirrers are stopped and phase separation is awaited. Afterwards, samples are taken which are typically analyzed by chromatographic methods (Diender et al., 2002; Gröger et al., 2004; Rosche et al., 2004; Villela-Filho et al., 2003). The experimental effort for those invasive studies could be reduced by using non-invasive measurement methods. In particular, spectroscopic techniques would allow to obtain quasi-continuous measurement data directly in emulsions under conditions close to those of large scale applications.

A spectroscopic method using a dispersed heterogeneous system consisting of water, immobilized enzyme, and insoluble substrate was successfully applied to determine enzyme kinetic parameters (Galunsky et al., 1994). Therefore, it is investigated if a similar method can be applied for emulsified systems. 


\subsubsection{Materials and methods}

To enable the application of spectroscopic measurements, a photometric cuvette was used as reactor. Emulsification experiments were performed using a magnetic stir bar on the cuvette bottom and an overhead stirrer. Eleven different kinds of stir bars were screened for their ability to emulsify the system. The overhead stirrer was designed in cooperation with Udo Kosfeld (electrical workshop). It can be plugged into the cuvette from the top and the stirring shaft can be exchanged. Stirrers with a shaft length from 15-25 mm and with a blade diameter from 3-7 mm were used. The stirring speed was varied between 0 and 13,000 rpm using a voltage regulator. The calibration between voltage and stirring speed was performed using a Voltcraft laser tachometer (Conrad Electronic SE, Hirschau, Germany).

The emulsification of four organic solvents with an aqueous phase was tested. For this purpose, $n$-hexane, cyclohexanone, methyl-iso-butly-ketone (MIBK), and ethyl-acetate were chosen. The aqueous phase consisted of $50 \mathrm{mM} \mathrm{KH} \mathrm{PO}_{4} / \mathrm{K}_{2} \mathrm{HPO}_{4}$ buffer and 25\%(v/v) DMF. The $\mathrm{pH}$ value was adjusted to 8.5 and the ionic strength to $100 \mathrm{mM}$. The studied reaction was the stereoselective carboligation of two 3,5-dimethoxy-benzaldehyde (DMBA) molecules to 3,3',5,5'-tetramethoxy-benzoin (TMB) using the enzyme benzaldehyde lyase (BAL, EC 4.1.2.38) from Pseudomonas fluorescens. The concentration of the cofactors $\mathrm{MgSO}_{4}$ and ThDP was $0.25 \mathrm{mM}$. The temperature was kept constant at $25^{\circ} \mathrm{C}$. Moreover, the surfactants Tween 20, Tween 65, and Span 80 were tested. All reagents were of analytical grade and were obtained from either Sigma-Aldrich (Deisenhofen, Germany) or from Carl Roth (Karlsruhe, Germany).

Fluorescence was chosen as spectroscopic technique. For this purpose the fluorimeter FP 6300 (Jasco, Tokyo, Japan) was used. The excitation wavelength was $360 \mathrm{~nm}$, the emission wavelength $470 \mathrm{~nm}$. The intensity loss caused by scattering at the droplets interface was considered using Eq. 5-14, where $I_{\text {emulsion }}$ and $I_{\text {hom }}$ are the intensities in the emulsion and in the homogeneous system, respectively. $\eta$ considers the intensity loss due to scattering and was determined for each emulsification condition individually.

$$
I_{\text {emulsion }}=I_{\text {hom }} \cdot \eta
$$




\subsubsection{Results and discussion}

The density difference between water and the organic solvent is crucial for emulsification. Organic solvents with low densities like $n$-hexane $\left(0.65 \mathrm{~kg} \mathrm{~L}^{-1}\right)$ cannot be emulsified using the applied setup, whereas emulsification of MIBK $\left(0.80 \mathrm{~kg} \mathrm{~L}^{-1}\right)$, ethyl-acetate $\left(0.90 \mathrm{~kg} \mathrm{~L}^{-1}\right)$, and cyclohexanone $\left(0.95 \mathrm{~kg} \mathrm{~L}^{-1}\right)$ is possible. Addition of surfactants considerably improves the ability for emulsification of organic solvents in water. Thus, even $n$-hexane can be emulsified. Surfactants lower the interfacial tension, therefore reducing the positive free energy change of dispersion related to surface formation. However, surfactants can complicate downstream processing (Fernandes and Cabral, 2008). Moreover, the emulsion becomes turbid which is in conflict with optical measurement techniques. For this reason, no surfactants are used for the kinetic experiments.
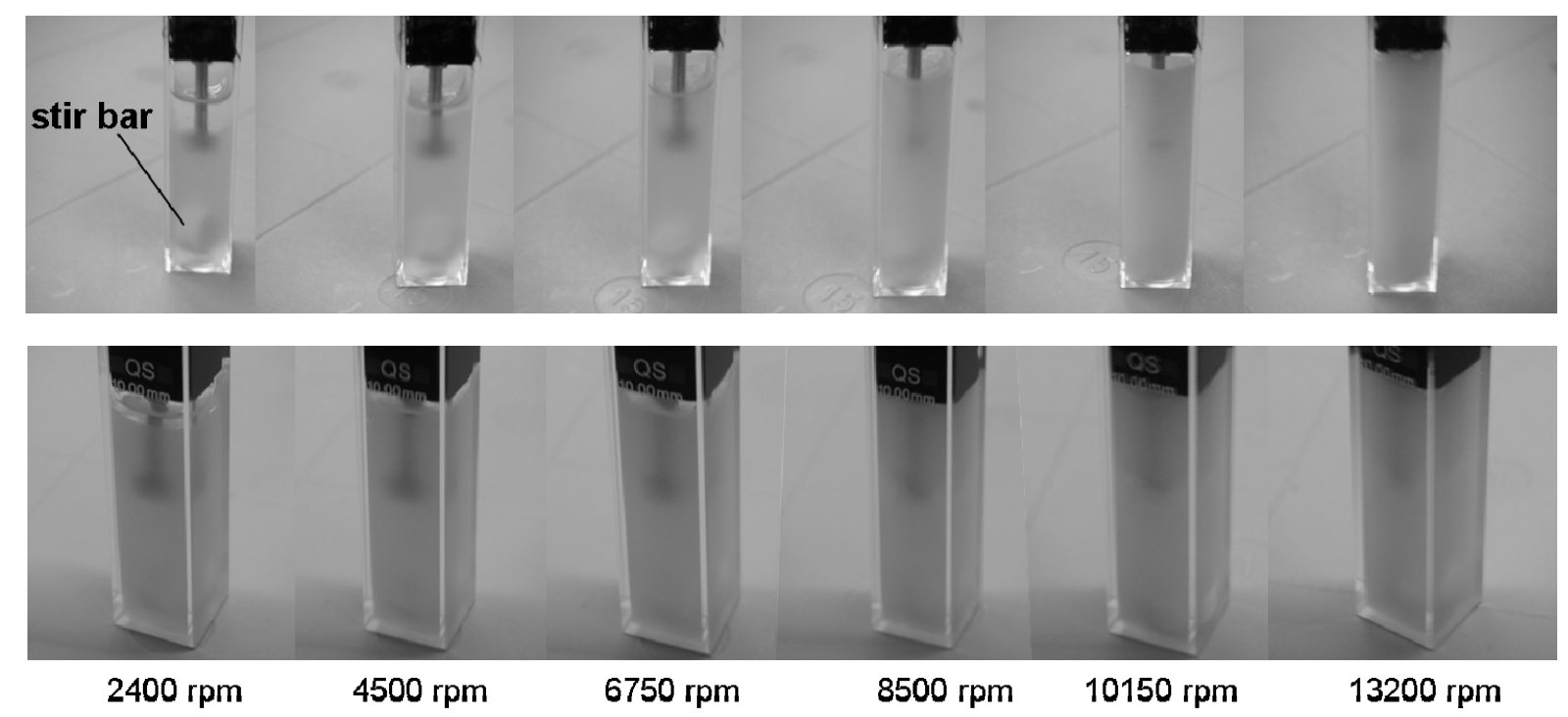

Figure 5-7: $\quad$ Emulsification experiment using the vertical disc stir bar and two different overhead stirrers. The upper row shows results for a blade stirrer with $3 \mathrm{~mm}$ diameter, the lower row for a blade stirrer with $7 \mathrm{~mm}$ diameter. The shaft length was in each case $25 \mathrm{~mm}$. The rotation frequencies of the overhead stirrers were increased from left to right. The rotation frequency of the vertical stir bar was kept constant at $725 \mathrm{rpm}$. MIBK was used as organic solvent. The aqueous phase was colored using $\mathrm{CuSO}_{4} . V_{\text {org }}=1 \mathrm{~mL}, V_{a q}=2 \mathrm{~mL}$.

To emulsify the biphasic system without surfactants in a cuvette, both a magnetic stir bar on the bottom and an overhead stirrer on top are required. Best results are obtained using the vertical disc stir bar (VWR, Darmstadt, Germany) at a maximum rotating frequency of $725 \mathrm{rpm}$. For the overhead stirrer a blade stirrer with $7 \mathrm{~mm}$ diameter and $25 \mathrm{~mm}$ shaft length is most suitable since it allows emulsification with the lowest rotating frequency $n$ 
(2400 rpm). For other types of stirrers higher rotating frequencies than $2400 \mathrm{rpm}$ are required to obtain complete emulsification as shown in Figure 5-7.

A volume fraction $\left(V_{\text {org }} / V_{a q}\right)$ of 0.5 is optimal for the applied setup, because in this case the overhead stirrer is close to the interface. Systems with different volume fractions require higher rotating frequencies. Considering the results of the $a b$ initio solvent screening (Chapter 3) MIBK was chosen as organic solvent since ethyl-acetate and cyclohexanone possess lower values for $R$ (Table 3-1).

Before enzyme kinetics in emulsions are studied, the mass transfer in the absence of the enzyme BAL is investigated. In emulsions the size distribution of droplets is difficult to determine (Fernandes and Cabral, 2008). Thus, the mass transfer area $A$ and the mass transfer coefficient $k_{i}$ are combined to one single parameter. Figure 5-8 shows the fit of the kinetic model to mass transfer experiments using DMBA.

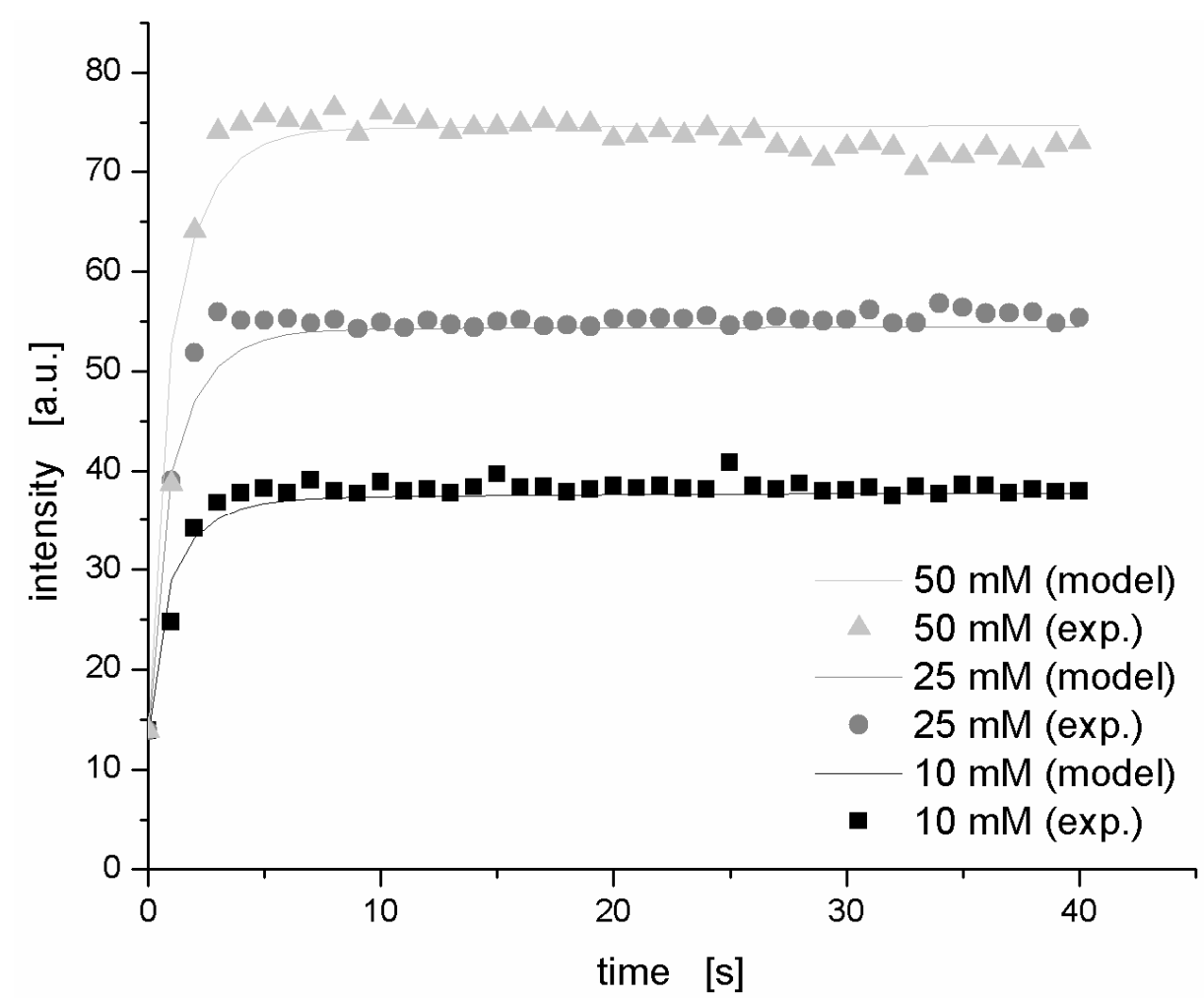

Figure 5-8: Mass transfer experiments for DMBA in emulsions. DMBA was added in different concentrations to the organic MIBK phase $\left(V_{\text {org }}=1 \mathrm{~mL}, V_{a q}=2 \mathrm{~mL} ; n_{\text {overhead stirrer }}=2400 \mathrm{rpm}\right.$, $\left.n_{\text {vertical disc stir bar }}=725 \mathrm{rpm}\right)$. 
According to Figure 5-8 the mass transfer in emulsions is so fast that equilibrium is achieved within five seconds. The obtained combined mass transfer coefficient $k_{A} \cdot A$ (Table 5-7) is about two orders of magnitude higher than in the Lewis cell $\left(1.7 \cdot 10^{-10} \mathrm{~m}^{3} \mathrm{~s}^{-1}-\right.$ Chapter 3$)$. The partition coefficient is in good agreement with previous results (Table 5-5). Since the quantum yield for fluorescence of TMB is much lower compared to DMBA, the signal to noise ratio in product mass transfer experiments is very low. Therefore, no reliable parameter values can be estimated. Instead, for the subsequent simulations it is assumed that the ratio of mass transfer coefficients is the same as in Chapter 3 . Thus for $k_{P} \cdot A$ a value of $1.010^{-8} \mathrm{~m}^{3} \mathrm{~s}^{-1}$ was assumed for the subsequent calculations.

Table 5-7: $\quad$ Obtained partition and mass transfer coefficient for the substrate in the emulsion.

\begin{tabular}{llll}
\hline Parameter & Unit & Estimate & Standard deviation \\
\hline$P_{A}$ & - & 54.0 & 2.0 \\
$k_{A} \cdot A$ & $\mathrm{~m}^{3} \mathrm{~s}^{-1}$ & $1.8 \cdot 10^{-8}$ & $0.4 \cdot 10^{-8}$ \\
\hline
\end{tabular}

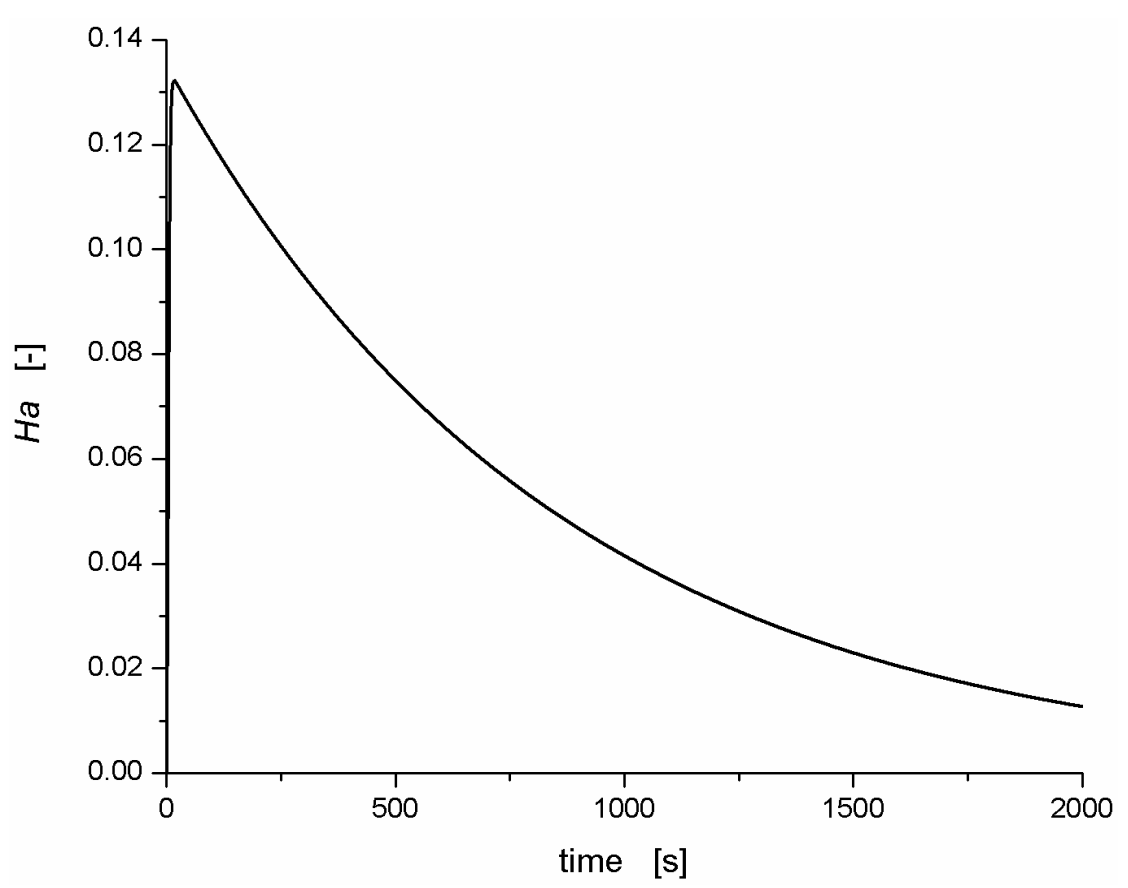

Figure 5-9: $\quad$ Calculated time course of the Hatta number $H a$ in the emulsified system assuming that the nominal values for the enzyme kinetic parameters (Table 5-2) are valid $\left(V_{\text {org }}=1 \mathrm{~mL}\right.$, $\left.V_{a q}=2 \mathrm{~mL} ; c_{E}=1.02 \cdot 10^{-4} \mathrm{mM} ; c_{A, \text { org }, 0}=5 \mathrm{mM} ; c_{A, a q, 0}=c_{P, \text { org }, 0}=c_{P, a q, 0}=0\right)$.

The enhanced mass transfer rate avoids mass transfer limitation in a reactive biphasic system. As discussed in Section 5.1.4 a Hatta-number $\mathrm{Ha}$ lower than 0.3 indicates a reaction 
limitation. Based on the results of Table 5-7 and the nominal values for the enzyme reaction (Table 5-5), $\mathrm{Ha}$ is calculated in Figure 5-9. Although the enzyme concentration of $1.02 \cdot 10^{-4} \mathrm{mM}$ is approximately two orders of magnitude higher than in the non-emulsified system (Table 5-4), the low $H a$ values clearly indicate that the process is carried out in the reaction limited regime. Thus, the concentrations in both phases can be assumed to be in mass transfer equilibrium at all times (Levenspiel, 1972). Before the corresponding experiment is conducted, the experiment is carried out in-silico (Figure 5-10).

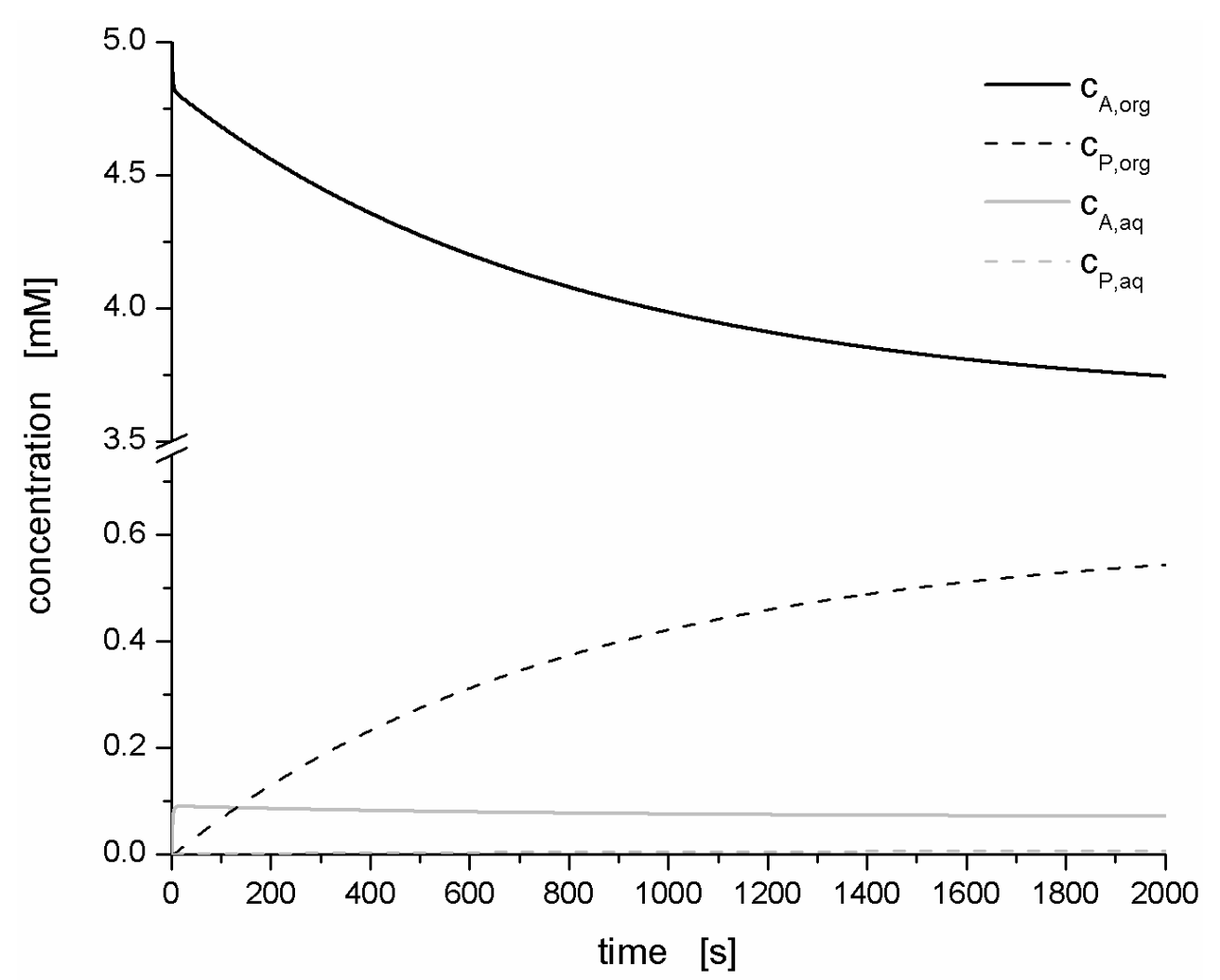

Figure 5-10: Simulated concentration profiles for the reactive emulsion assuming that the nominal values for the enzyme kinetic parameters (Table 5-2) are valid $\left(V_{\text {org }}=1 \mathrm{~mL}, V_{a q}=2 \mathrm{~mL}\right.$; $\left.c_{E}=1.02 \cdot 10^{-4} \mathrm{mM} ; c_{A, \text { org }, 0}=5 \mathrm{mM} ; c_{A, a q, 0}=c_{P, o r g, 0}=c_{P, a q, 0}=0\right)$.

From Figure 5-10 it can be deduced that $c_{P, a q}$ remains very low (it is very close to the axis of abscissae) since the product is immediately extracted into the organic phase. Thus, the influence of $c_{P, a q}$ on the measured fluorescence intensity can be neglected. For the other three species, $A_{\text {org }}, A_{a q}$, and $P_{\text {org }}$, the fluorescence intensity is quantified in separate experiments. $A_{\text {org }}$ does not show any fluorescence, while the measured intensity of $5 \mathrm{mM} A_{a q}$ is 412.7 arbitrary units (a.u.). The fluorescence intensity of $5 \mathrm{mM} P_{\text {org }}$ is 22.3 a.u. resulting in a fluorescence ratio $I_{A, a q} / I_{P, \text { org }}$ of 18.5. As shown in Figure 5-10, the final concentration of $P_{\text {org }}$ is approximately six times higher than the final concentration of $A_{a q}$. Therefore, the 
proportion of $P_{\text {org }}$ of the total fluorescence intensity reaches one third. For this reason, it cannot be assumed that the fluorescence intensity depends only on $c_{A, a q}$. Varying the excitation and emission wavelengths does not reduce the superposition of the fluorescence intensities of $A_{a q}$ and $P_{\text {org. }}$. Consequently, fluorescence measurements do not allow determining one concentration independently and thus the kinetic parameters cannot be identified for the investigated reaction system.

Another drawback of the reactive emulsion is that in the end of the conducted experiments aggregates can be observed. This is in contrast to the mass transfer experiments. Since the only difference is the presence of enzyme, this indicates enzyme aggregation. Moreover, this assumption is strengthened by the observation that more aggregates are formed, if the enzyme concentration is increased (Figure 5-11). A possible reason is that enzymes adsorb to the interface, unfold and irreversibly form aggregates (Baldascini and Janssen, 2005). Although phase toxicity was not observed in the Lewis cell (Section 5.1), it becomes significant in the emulsified system for three reasons: First the interface is strongly enlarged due to emulsification. Second the enhanced agitation increases the probability that enzymes come into contact with the interface. Third aggregation of enzymes can be considered as a reaction of second order, which is dramatically accelerated in the emulsified system since the enzyme concentration is about two orders of magnitude higher than in the Lewis cell. In order to avoid phase toxicity, immobilization of enzymes is advisable (Halling, 1987).

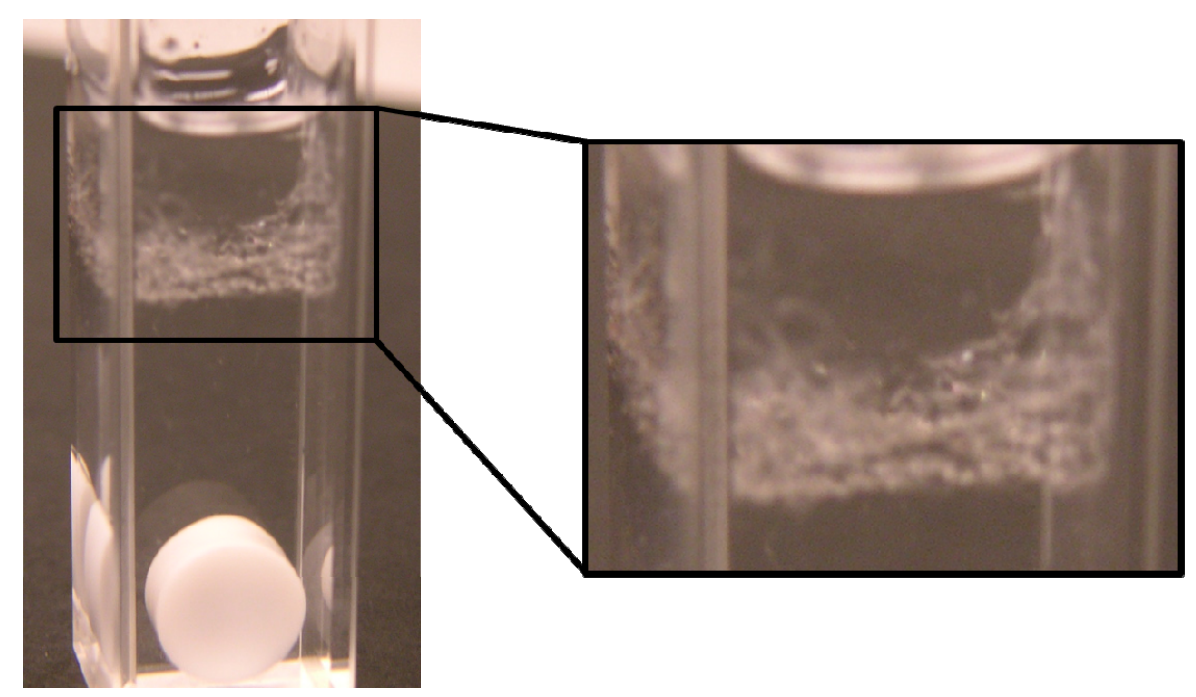

Figure 5-11: Aggregates formed after emulsification of the biphasic system in presence of enzyme $\left(V_{\text {org }}=1 \mathrm{~mL}, V_{a q}=2 \mathrm{~mL} ; c_{E}=5.75 \cdot 10^{-4} ; c_{A, o r g, 0}=25 \mathrm{mM} ; c_{A, a q, 0}=c_{P, o r g, 0}=c_{P, a q, 0}=0\right)$. 


\subsubsection{Conclusions}

In order to avoid mass transfer limitation the emulsification of an aqueous-organic biphasic reaction system may be beneficial. The mass transfer is significantly enhanced due to increased agitation and thus higher volumetric productivities can be achieved. However, reactive emulsions are difficult to analyze in small scale. In previous studies, emulsification was interrupted repeatedly and samples were taken. In this Section an alternative approach to these invasive methods was investigated. A biphasic system was emulsified in a photometric cuvette using a magnetic vertical disc stir bar and a newly developed overhead stirrer. This setup allows non-invasive spectroscopic measurements during emulsification. It was demonstrated that the enantioselective coupling of two DMBA molecules to TMB can be carried out in the reaction-limited regime using the applied setup. However, the superposition of fluorescence signals of DMBA in the aqueous phase and of TMB in the organic phase impedes the determination of the kinetic parameters. Another drawback of a reactive emulsion is that enzyme deactivation due to phase toxicity may be significantly enhanced. In the present study, formation of aggregates could be observed which indicates enzyme deactivation.

However, the developed setup may be applied for other enzymatic reaction systems. The hydrolysis of 6-nitro-3-phenylacetamido benzoic acid using penicillin amidase (EC 3.5.1.11) from E. coli has already been investigated (results not shown). Moreover, the reduction of acetophenon to phenylethanol using alcohol dehydrogenase (EC 1.1.1.2) from Lactobacillus brevis was studied (results not shown). In these systems the main drawback is that the measured extinctions are strongly superimposed by scattering of droplets. To date, no reliable estimation of kinetic parameters in emulsions using non-invasive spectroscopic measurements is possible. 


\section{Coupling of Diffusion and Mass Transfer Kinetics}

\subsection{Introduction}

To approach the final gel-stabilized aqueous-organic reactive system, the kinetic models for the occurring phenomena are successively combined. While in the previous chapter the kinetic models for the enzyme reaction and the mass transfer were coupled, this chapter addresses the coupling of diffusion inside the hydrogel and mass transfer between the aqueous and the organic phase. In Chapter 4 the diffusion of the substrate 3,5-dimethoxybenzaldehyde (DMBA) and of the product $(R)-3,3$ ',5,5'-tetramethoxy-benzoin (TMB) in $\kappa$-carrageenan hydrogel was investigated using two photon laser scanning microscopy (LSM) and ${ }^{1} \mathrm{H}$ nuclear magnetic resonance (NMR) spectroscopy. In those investigations an aqueous bulk phase was chosen so that the partitioning of the reactants between an organic phase and the aqueous hydrogel had not to be considered. However, an organic bulk phase may improve product extraction. Thus, the effects of the presence of an organic bulk are investigated in this chapter. For this purpose, the model-based experimental analysis (MEXA) approach is followed (Marquardt, 2005). Accordingly, a kinetic model is derived, which considers diffusion inside the hydrogel and mass transfer at the interface. The kinetic model permits a priori simulations, sensitivity analysis, and optimal experimental design. The model parameters are estimated by fitting the kinetic model to experimental data.

\subsection{Model development}

The combined kinetic model for diffusion and mass transfer consists of the same model equations as the diffusion model (Chapter 4). The only difference is the incorporation of the partition coefficient $P_{i}$ at the interface. Eqs. 6-1 and 6-2 are the mass balances for the bulk and the hydrogel bead, wherein $V_{\text {bulk }}$ is the bulk volume, $c_{i, b u l k}$ and $c_{i \text {,bead }}$ are the concentrations of the species $i$ in the bulk and in the bead, respectively. The mass transfer coefficient is denoted by $k_{i}, A_{\text {bead }}$ is the surface of the bead, $R$ the bead radius, and $j_{i}$ the molar diffusive flux: 


$$
\begin{gathered}
V_{\text {bulk }} \frac{d c_{i, \text { bulk }}}{d t}=k_{i} \cdot A_{\text {bead }} \cdot\left(P_{i} \cdot c_{i, \text { bead }}(r=R)-c_{i, \text { bulk }}\right) \\
\frac{\partial c_{i, \text { bead }}}{\partial t}=-\frac{1}{r^{2}}\left(\frac{\partial}{\partial r} \cdot r^{2} \cdot j_{i}\right)
\end{gathered}
$$

As discussed in Chapter 4.2.2, no electrostatic interactions have to be considered in terms of the Nernst-Planck diffusion law since DMBA and TMB are uncharged species. Thus, Fick's diffusion law is used (Eq. 6-3). The effective diffusion coefficient $D_{i, \text { eff }}$ takes into account the influence of the hydrogel material on the diffusion rate.

$$
j_{i}=-D_{i, e f f} \cdot \frac{\partial c_{i}}{\partial r}
$$

The combined model contains the following boundary and initial conditions:

$$
\begin{gathered}
j_{i}(r=0)=0 \\
j_{i}(r=R)=k_{i} \cdot\left(P_{i} \cdot c_{i, \text { bead }}(r=R)-c_{i, \text { bulk }}\right) \\
c_{i, \text { bulk }}(t=0)=c_{i, \text { bulk }, 0} \\
c_{i, \text { bead }}(t=0)=c_{i, \text { bead }, 0}
\end{gathered}
$$

\subsection{A priori simulations}

The combined kinetic model for diffusion and mass transfer is used for a priori simulations. As organic solvent methyl-iso-butyl-ketone (MIBK) is chosen. The nominal model parameters (Table 6-1) were obtained from the separate models in Chapters 3 and 4. For the a priori simulations it is assumed that no mass transfer limitation exists. Although this assumption has been verified for an aqueous bulk in Chapter 4.2, it has to be checked for the organic bulk phase later on in the parameter estimation (Section 6.6). 
Table 6-1: $\quad$ Nominal values for the model parameters. The values were determined in Chapters 3 and 4.

The partition coefficients refer to MIBK as organic solvent.

\begin{tabular}{lll}
\hline parameter & nominal value & unit \\
\hline$D_{A, \text { eff }}$ & $4.139 \cdot 10^{-10}$ & $\mathrm{~m}^{2} \mathrm{~s}^{-1}$ \\
$D_{P, \text { eff }}$ & $2.261 \cdot 10^{-10}$ & $\mathrm{~m}^{2} \mathrm{~s}^{-1}$ \\
$P_{A}$ & 52.37 & - \\
$P_{P}$ & 84.36 & - \\
\hline
\end{tabular}

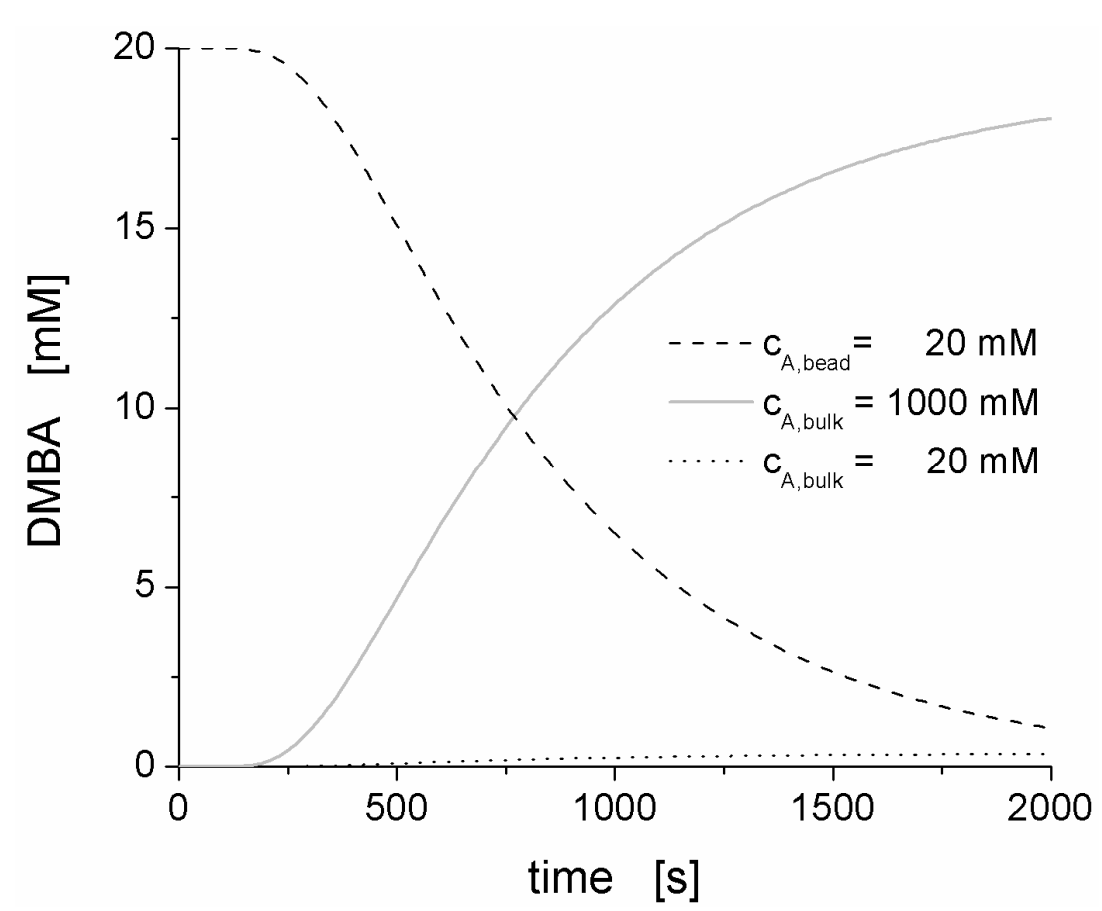

Figure 6-1: A priori simulations of the DMBA concentration in the bead center. Three test cases are investigated: Case 1: Addition of $20 \mathrm{mM}$ DMBA to the bulk phase (dotted line). Case 2: Addition of $1000 \mathrm{mM}$ DMBA to the bulk phase (solid line). Case 3: Addition of $20 \mathrm{mM}$ DMBA to the hydrogel bead (dashed line) (all other concentrations are zero, $R=1.5 \mathrm{~mm}$ ).

Figure 6-1 shows the results of the a priori simulations of the DMBA concentration in the bead center for three different cases. If for example $20 \mathrm{mM}$ DMBA is added to the bulk phase, just like in the experiments described in Chapter 4, the concentration in the bead remains very low. The reason for this is the high partition coefficient. Low bead concentrations cause low signal to noise ratios. Thus, higher bead concentrations are beneficial. In order to achieve sufficiently high bead concentrations, bulk concentrations in the range of $1 \mathrm{M}$ are required. However, these concentrations are above the solubility limits of the reactants. The alternative is to add the substances to the bead. As shown in Figure 6-1, this 
leads to a significant decrease of the concentration in the bead over time since the molecules diffuse into the bulk. As a result the signal to noise ratio is strongly enhanced. Moreover, less amount of substance is required since it has to be added only to the small bead and not to the large bulk. For these reasons, this approach is applied for the subsequent investigations.

\subsection{Sensitivity analysis}

In Chapter 4 it was demonstrated that diffusion measurements should be performed by measuring the concentration in the bead center using non-invasive spectroscopic measurements. However, in those investigations the diffusing substance was added to the bulk phase. Figure 6-2 shows the result of a sensitivity analysis for the case that the diffusing substance is added to the hydrogel bead. The normalized sensitivities $S_{i, j}$ were calculated using Eq. 6-8 and approximated using finite differences. $\theta_{j}$ denotes the parameter $j$ and $c_{i}$ the concentration of compound $i$.

$$
S_{i, j}=\frac{\theta_{j}}{c_{i}} \cdot \frac{\partial c_{i}}{\partial \theta_{j}}
$$

According to Figure 6-2 the sensitivity maximum of the diffusion coefficient is about one order of magnitude higher if the DMBA concentration is measured in the bead center compared to bulk measurements. Moreover, the estimation of the diffusion coefficient in the bulk phase is strongly superimposed by the influences of bead radius and bulk volume. In contrast to this, the influence of the bulk volume is negligible if the measurement is performed in the bead center. However, the bead radius has to be accurately determined. This is achieved by separately measuring the bead radius after each experiment as described in Chapter 4. Moreover, Figure 6-2 shows that the sensitivity of the partition coefficient is very low in both cases and thus the partition coefficient cannot be estimated with sufficient precision using the two compared experimental strategies. 

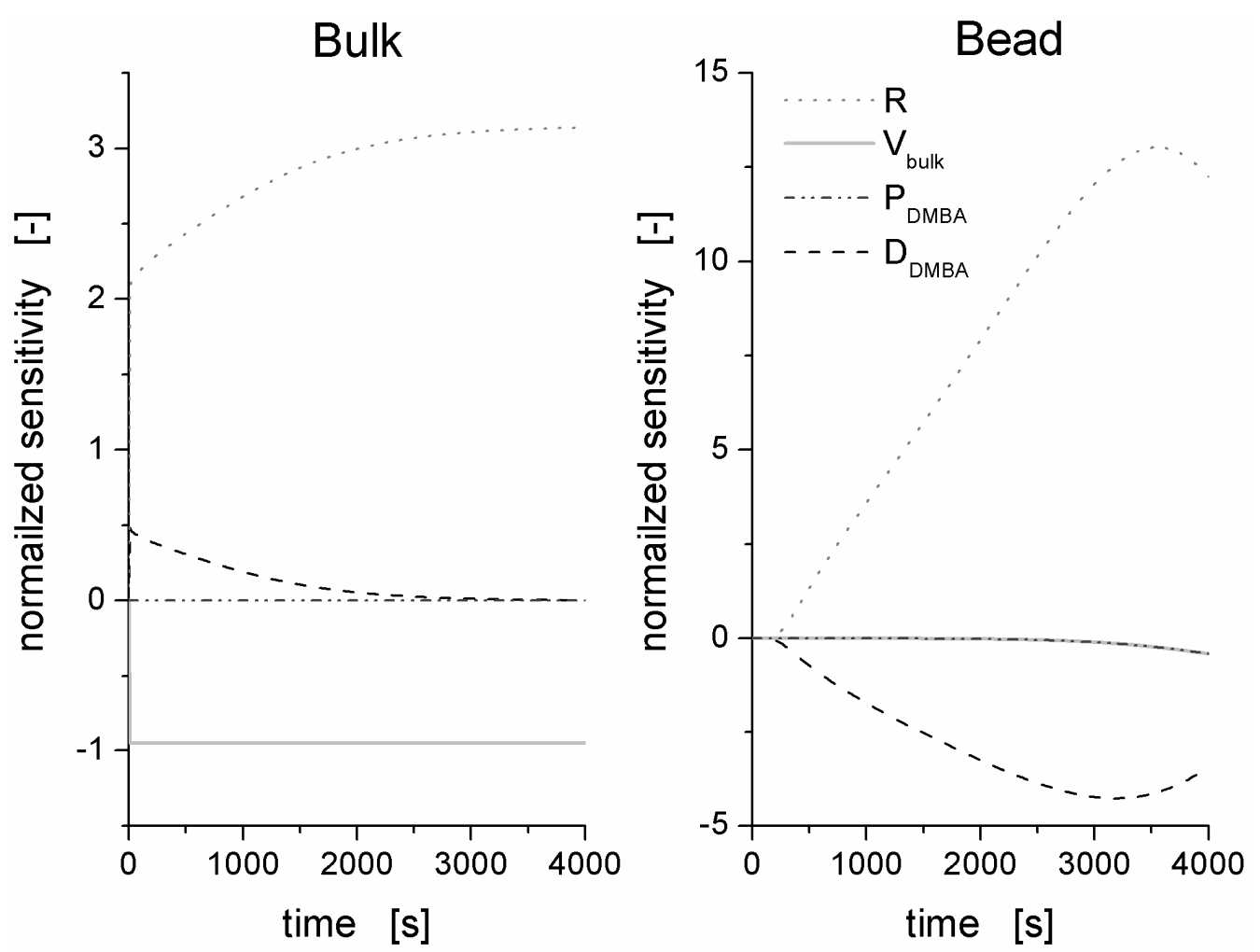

Figure 6-2: $\quad$ Sensitivity analysis for the diffusion coefficient of DMBA $D_{A, \text { efff, }}$, the bulk volume $V_{b u k}$, the bead radius $R$, and the partition coefficient of DMBA $P_{A}$ in the bulk phase (left) and in the bead center (right). The sensitivity refers to the DMBA concentration. The nominal values of the parameters are listed in Table 6-1 $\left(c_{A, b e a d, 0}=20 \mathrm{mM}, R=1.5 \mathrm{~mm}, V_{\text {bulk }}=250 \mu \mathrm{L}\right)$.

\subsection{Materials and methods}

The same chemicals as described in Chapters 3 and 4 were used. All of them were of analytical grade. The preparation of the $\kappa$-carrageenan hydrogel beads was explained in Chapter 4.2.4. In order to adjust the initial concentration of the diffusing substance in the hydrogel beads, the beads were equilibrated with an excessive amount of aqueous solution consisting of the intended concentration of DMBA, $50 \mathrm{mM} \mathrm{KH}_{2} \mathrm{PO}_{4} / \mathrm{K}_{2} \mathrm{HPO}_{4}$, and $25 \%(\mathrm{v} / \mathrm{v})$ dimethylformamide (DMF). The diffusion experiments were performed in a 96-well microtiter plate at room temperature. The hydrogel bead was placed on top of a circular holder to guarantee uniform conditions. The experiments were started by the addition of $250 \mu \mathrm{L}$ of MIBK to the well. In order to avoid solvent evaporation, the well of the microtiter plate was sealed. 
The diffusion experiments were conducted using a two photon LSM. Details regarding this device can be found in Chapter 4.2.4. The excitation wavelength for both DMBA and TMB was $723 \mathrm{~nm}$, which is approximately the doubled wavelength of their excitation maximum due to the two photon process. For both substances all photons with a wavelength below the excitation wavelength were collected by a photomultiplier (H7422P-40, Hamamatsu Photonics GmbH, Herrsching, Germany). For calibration hydrogel beads were equilibrated with different concentrations of DMBA and TMB, respectively. Using the measured fluorescence intensities, linear calibration curves were obtained. For each measurement series a new calibration curve was determined.

After each measurement, the correct position and the quality of the hydrogel bead were controlled using light microscopy. If they were not satisfactory, the respective data were not considered. According to the results of the sensitivity analysis, the bead radius was optically measured using the method of Buthe et al. (2004). This also allows the determination of the roundness $\left(R_{\min } / R_{\max }\right)$. Beads with a roundness less than 0.8 were disregarded.

\subsection{Results and discussion}

The coupled kinetic model for diffusion and mass transfer was fitted to 12 experiments using DMBA and 10 experiments using TMB. According to the results of the previous Sections, the diffusion of the substances from the hydrogel bead into the organic bulk phase was studied by measuring their concentration at the bead center. For parameter estimation a sequential approach was followed. In a first step, the parameters bead radius $R$, the initial concentration in the bead $c_{i, b u l k, 0}$, and the lag-time $t_{l a g}$, i.e. the time interval between starting the measurement and adding the bulk phase, were individually estimated for each experiment. $R$ and $c_{i, b u l k, 0}$ were estimated in bounds of $\pm 10 \%$ around the nominal values, $t_{\text {lag }}$ was estimated in bounds of $\pm 10 \mathrm{~s}$ around the nominal starting time. In the second step, mass transfer and diffusion coefficients were estimated. Due to the low sensitivity of the partition coefficients using the experimental setup (Section 6.4), these parameters were not included into the parameter estimation. Instead the values obtained in Chapters 3 and 4 and listed in Table 6-1 were used. Figures 6-3 and 6-4 show the fits of the kinetic model to the experimental data. 


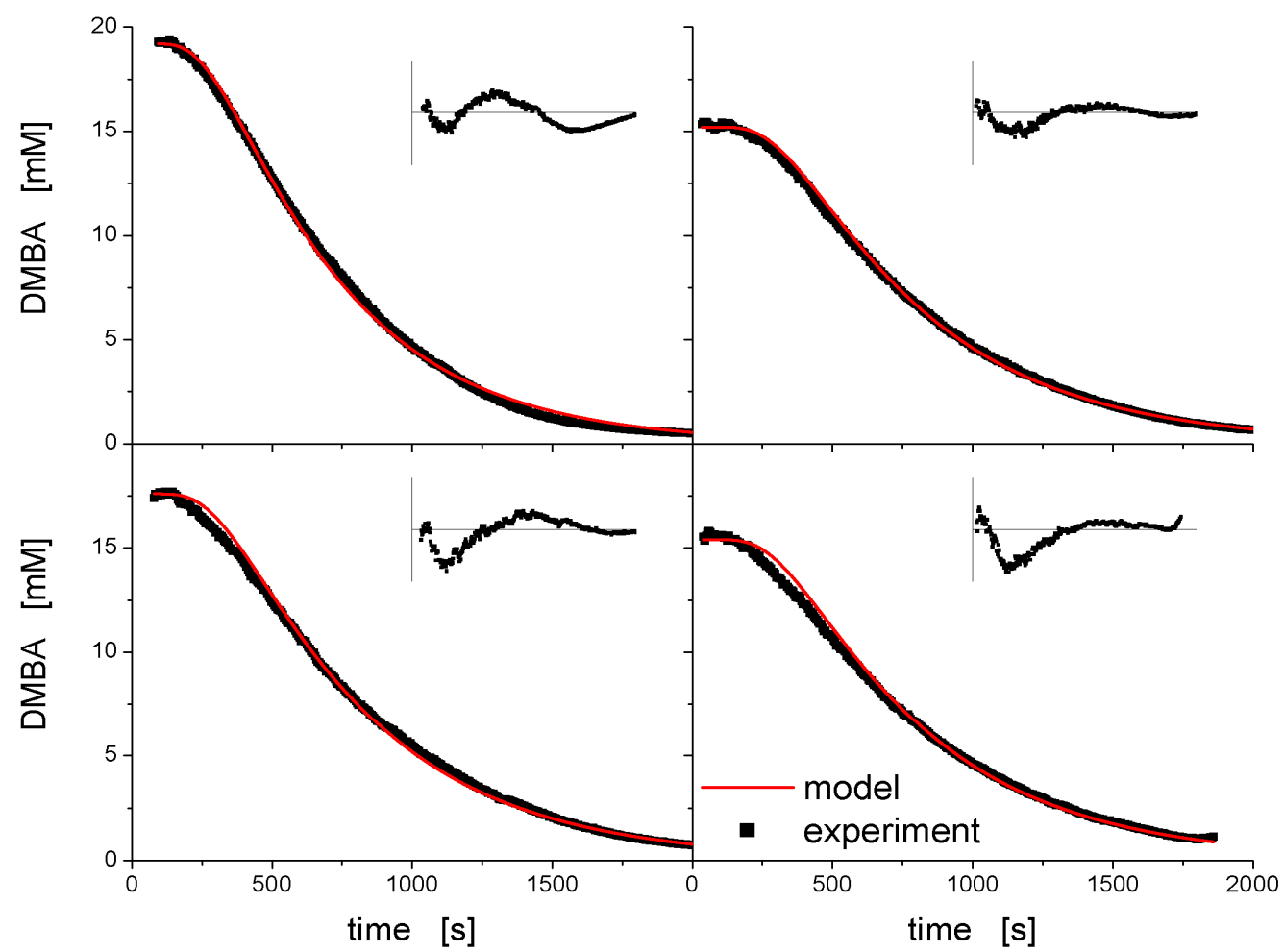

Figure 6-3: Comparison of the fitted kinetic model for diffusion and mass transfer with the experimental data for DMBA diffusion. 4 of 12 fits are shown as example. The residuals are shown as small inlets (nominal conditions: $c_{A, g e l, 0}=15-20 \mathrm{mM}, R=1.5 \mathrm{~mm}$ ).

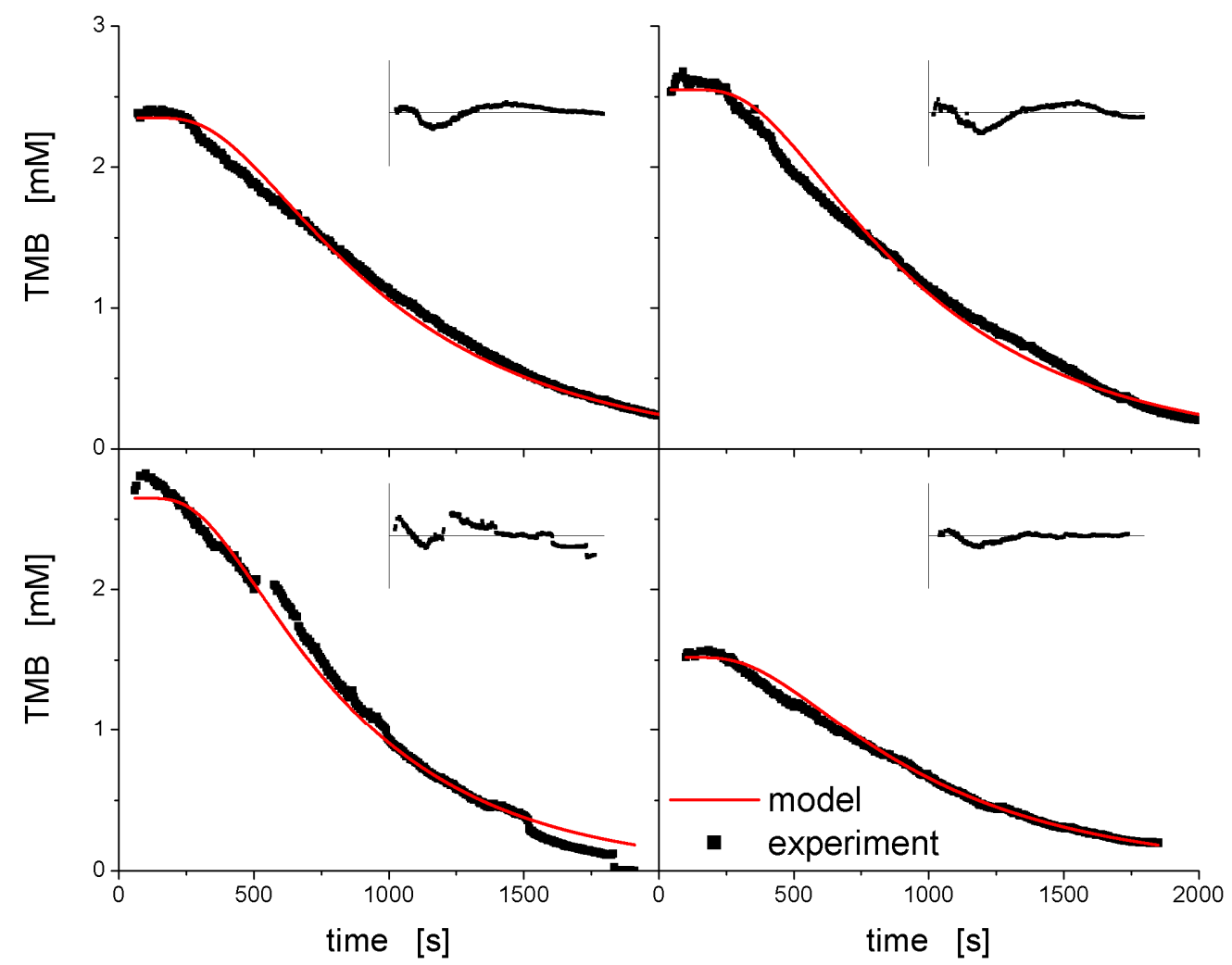

Figure 6-4: Comparison of the fitted kinetic model for diffusion and mass transfer with the experimental data for TMB diffusion. 4 of 10 fits are shown as example. The residuals are shown as small inlets (nominal conditions: $c_{P, g e l, 0}=2-3 \mathrm{mM}, R=1.5 \mathrm{~mm}$ ). 
The kinetic model fits the experimental data for the diffusion of DMBA satisfactorily (Figure 6-3). As observed already in Chapter 4.2.5, all residual plots indicate a minor systematic deviation in the beginning of the diffusion process. Obviously, the decrease of the DMBA concentration starts earlier than the kinetic model predicts. The reason for this may be that the beads are not perfectly spherical as assumed in the kinetic model. Thus, the minimal distance to the interface is shorter and the concentration in the bead center is affected earlier by the diffusion. These differences can also be seen for TMB diffusion (Figure 6-4). Due to the limited solubility of TMB in water, a TMB concentration of about $3 \mathrm{mM}$ cannot be exceeded. Moreover, the quantum yield is much lower for TMB than for DMBA. Owing to these drawbacks, the signal to noise ratio is lower resulting in a lower quality of the experimental data compared to those for DMBA.

Table 6-2: $\quad$ Estimated effective diffusion coefficients of DMBA and TMB in $\kappa$-carrageenan hydrogel beads using MIBK as bulk phase.

\begin{tabular}{llll}
\hline parameter & estimate & standard deviation & unit \\
\hline$D_{A, \text { eff }}$ & $4.248 \cdot 10^{-10}$ & $2.862 \cdot 10^{-13}$ & $\mathrm{~m}^{2} \mathrm{~s}^{-1}$ \\
$D_{P, \text { eff }}$ & $3.110 \cdot 10^{-10}$ & $7.781 \cdot 10^{-13}$ & $\mathrm{~m}^{2} \mathrm{~s}^{-1}$ \\
\hline
\end{tabular}

The estimated parameters are listed in Table 6-2. No reliable values can be obtained for the mass transfer coefficients (the estimates always hit the upper bounds), which indicates that the system is diffusion limited. If the values for the mass transfer coefficients are decreased to a level where mass transfer is limiting, the resulting curves show different behaviors and the model can no longer be fitted to the experimental data. This supports the aforementioned assumption of diffusion limitation. The estimated diffusion coefficient for DMBA is very close to the one obtained using an aqueous bulk phase $\left(4.139 \cdot 10^{-10} \mathrm{~m}^{2} \mathrm{~s}^{-1}\right.$ - Chapter 4.2.5). The deviation is only $2.6 \%$. This indicates that the occurrence of an organic bulk does not considerably affect the diffusion rate of DMBA. Moreover, it further supports the assumption of diffusion limitation since the mass transfer rate using an aqueous bulk and an organic bulk would probably be different. However, the situation changes for TMB. Here, the estimated value is significantly higher than the value obtained for an aqueous bulk $\left(2.261 \cdot 10^{-10} \mathrm{~m}^{2} \mathrm{~s}^{-1}\right.$ Chapter 4.2.5). In this case the deviation is $37.5 \%$. One explanation could be that minor amounts of dissolved organic solvent molecules in the hydrogel enhance the diffusion rate of 
TMB. However, due to the low quality of the TMB data, mechanistic conclusions should not be drawn. Instead, further investigations of this difference should be performed.

\subsection{Comparison with NMR}

In Chapter 4.3 the diffusion in hydrogel cylinders was studied using ${ }^{1} \mathrm{H}$ nuclear magnetic resonance (NMR) spectroscopy. Details about this measurement technique can be found in that chapter. One diffusion experiment for DMBA was performed using an aqueous bulk phase. In that case, the estimated diffusion coefficient for DMBA was approximately $40 \%$ lower than the one obtained using CLSM. One drawback was swelling of the $\kappa$-carrageenan hydrogel which was not considered in the kinetic model. However, if an organic bulk is used, no hydrogel swelling due to water uptake can occur. Thus, another NMR experiment is conducted using an organic bulk. In this case, $n$-hexane is chosen. The kinetic model for diffusion in a hydrogel cylinder, described in Chapter 4.3.3, is modified by introducing the partition coefficient $P_{i}$, which was previously determined (Chapter 3).

$$
c_{i, \text { bulk }}\left(z_{1}=0\right)=P_{i} \cdot c_{i, c y l i n d e r}\left(z_{2}=0\right)
$$

Figure 6-5 shows the fit of the kinetic model to the experimental data at different positions in the hydrogel cylinder. As already discussed in Chapter 4.3, NMR provides less data points than CLSM. Moreover, the data quality is lower. However, the fits are satisfactory and the diffusion coefficients can be estimated precisely (Table 6-3).

The estimated diffusion coefficient of DMBA in $n$-hexane is about 20 times higher than in the hydrogel. Reasons are the lower viscosity of $n$-hexane compared to water and a possible steric hindrance due to the hydrogel matrix. Moreover, vibrations in the NMR device might cause mixing of the bulk phase. Since this is not considered in the kinetic model, the diffusion coefficients would appear to be higher. The diffusion coefficient in the hydrogel is $63 \%$ higher than the one obtained with NMR using an aqueous bulk phase. However, the value is in good agreement with the estimated diffusion coefficients in the hydrogel beads using CLSM. Table 6-4 lists all obtained diffusion coefficients for DMBA in $\kappa$-carrageenan hydrogel for comparison. 


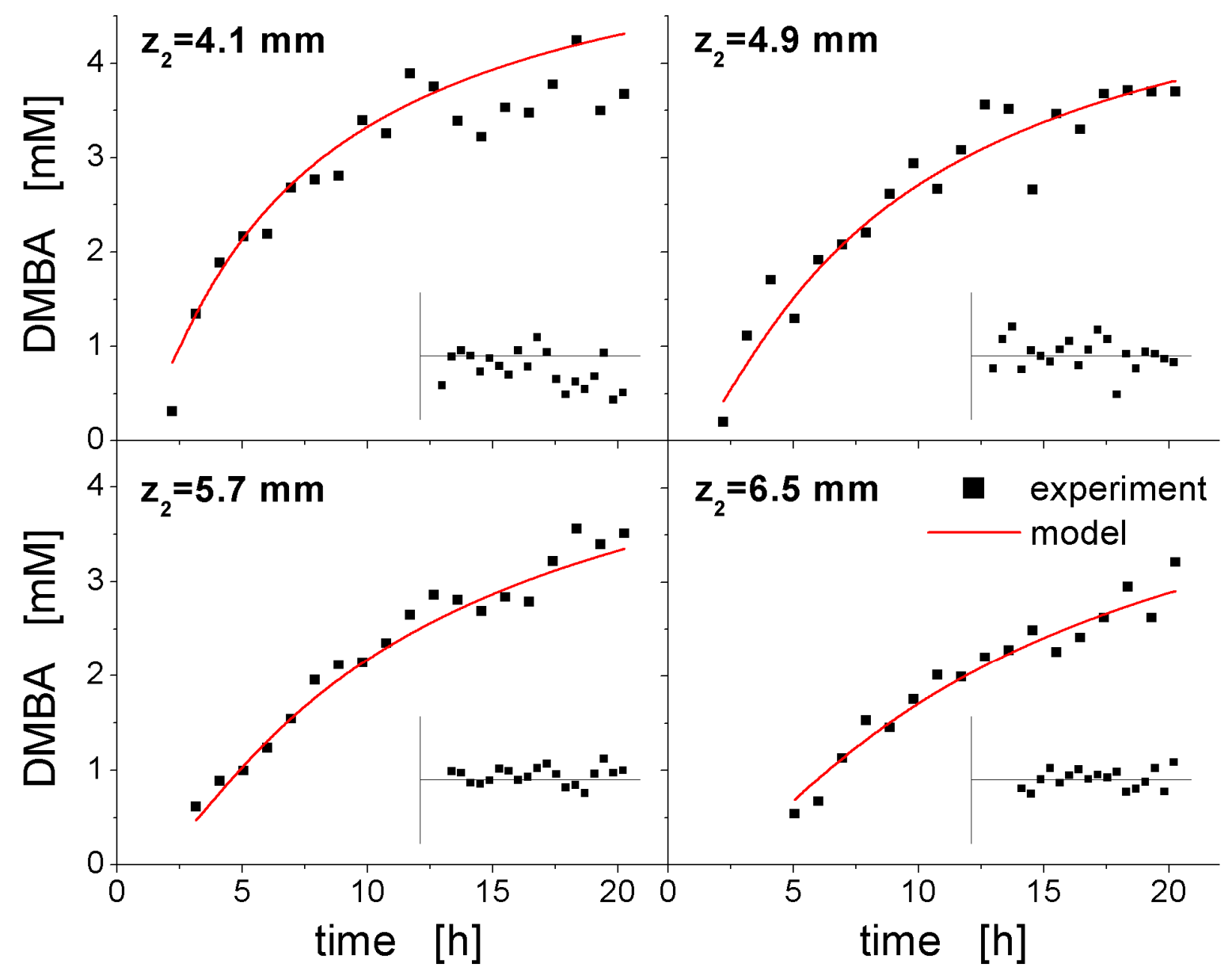

Figure 6-5: Comparison of the fitted kinetic model with the experimental data for DMBA diffusion at different positions in the hydrogel cylinder using $n$-hexane as organic bulk phase. The experimental data were obtained by ${ }^{1} \mathrm{H}-\mathrm{NMR}$. The residuals are shown as small inlets $\left(c_{A, \text { bulk }, 0}=25 \mathrm{mM}, L_{c y l i n d e r}=0.056 \mathrm{~m}, L_{b u l k}=0.025 \mathrm{~m}\right)$.

Table 6-3: $\quad$ Estimated diffusion coefficients of DMBA in the $n$-hexane bulk phase and in the $\kappa$-carrageenan hydrogel cylinders.

\begin{tabular}{llll}
\hline parameter & estimate & standard deviation & unit \\
\hline$D_{A, n-h e x a n e}$ & $8.303 \cdot 10^{-9}$ & $1.847 \cdot 10^{-9}$ & $\mathrm{~m}^{2} \mathrm{~s}^{-1}$ \\
$D_{A, \text { gel }}$ & $4.028 \cdot 10^{-10}$ & $5.939 \cdot 10^{-12}$ & $\mathrm{~m}^{2} \mathrm{~s}^{-1}$ \\
\hline
\end{tabular}


Table 6-4: Comparison of the estimated diffusion coefficients for DMBA in $\kappa$-carrageenan hydrogel (all values are in $\mathrm{m}^{2} \mathrm{~s}^{-1}$ ).

\begin{tabular}{llll}
\hline bulk & measurement technique & estimate & standard deviation \\
\hline \multirow{2}{*}{ aqueous } & CLSM & $4.139 \cdot 10^{-10}$ & $3.881 \cdot 10^{-13}$ \\
& NMR & $2.465 \cdot 10^{-10}$ & $2.250 \cdot 10^{-12}$ \\
\multirow{2}{*}{ organic } & CLSM & $4.248 \cdot 10^{-10}$ & $2.862 \cdot 10^{-13}$ \\
& NMR & $4.028 \cdot 10^{-10}$ & $5.939 \cdot 10^{-12}$ \\
\hline
\end{tabular}

Table 6-4 indicates that the estimated value using an aqueous bulk and NMR is an outlier. The main reason is that due to the longer duration of NMR experiments the hydrogel swells due to water uptake, which is not considered in the kinetic model. Water uptake can be suppressed either by using an organic bulk or by applying CLSM which requires lower measurement duration. Thus, the other three values are very close to each other. The fact that these three values are almost identical for different bulk phases (water, MIBK, and $n$-hexane) supports the finding that an organic bulk phase does not considerably affect the diffusion rate of DMBA in the hydrogel. Moreover, this indicates that the organic solvents do not interact with the $\kappa$-carrageenan hydrogel.

\subsection{Conclusions}

Suspending hydrogel beads with immobilized enzymes in an organic bulk phase may improve extraction of hydrophobic products. However, up to now it was unclear if the presence of an organic solvent affects the diffusion of the reactants in the hydrogel bead, e.g. by interacting with the hydrogel matrix. Therefore, in this chapter the diffusion of DMBA and TMB in $\kappa$-carrageenan hydrogel beads surrounded by an organic bulk phase was investigated. As organic solvent MIBK was chosen. The models for diffusion in the hydrogel and mass transfer between an aqueous and an organic phase were combined and fitted to experimental data obtained by CLSM. The results were compared to those from Chapter 4, where the diffusion in hydrogel beads without an organic bulk phase was studied. The combined kinetic model describes the experimental data satisfactorily. The estimated diffusion coefficient for DMBA in the hydrogel using MIBK as organic bulk phase is very close to the estimate using an aqueous bulk phase. This indicates that MIBK does not considerably affect the diffusion 
rate of DMBA in the hydrogel. However, the diffusion coefficient of TMB in the hydrogel was estimated to a higher value than the one obtained using an aqueous bulk phase. Since the quality of the experimental data for TMB is lower than for DMBA, further investigations are necessary. Furthermore, NMR was applied to measure the diffusion of DMBA in hydrogel cylinders. Here, $n$-hexane was used as bulk phase. The obtained diffusion coefficient in the hydrogel is in line with those obtained by CLSM. This supports the conclusion that the diffusion of DMBA in $\kappa$-carrageenan hydrogel is independent from the bulk phase and that the organic phase does not interact with the $\kappa$-carrageenan hydrogel. 


\section{Enzyme Kinetics in a Gel-Stabilized Aqueous- Organic Biphasic System}

\subsection{Introduction}

For the utilization of enzymes in aqueous-organic biphasic systems, immobilization in hydrogel beads may prevent enzyme deactivation due to phase toxicity. Moreover, enzyme immobilizates facilitate downstream processing and reduce the overall process costs since they can easily be recovered and reused (Ansorge-Schumacher, 2007; Cao, 2005; Fernandes and Cabral, 2008; Kurlemann and Liese, 2004; Mateo et al., 2007; Sheldon, 2007). The rational design of enzyme immobilizates is more complex than that of homogeneous systems since mass transfer and diffusion can be rate limiting (Bauer et al., 2002; Berendsen et al., 2006; Buchholz, 1989; Diender et al., 2002; Fernandes and Cabral, 2008; Halling, 1987, 1994). Usually it is assumed that the kinetic parameters of immobilized enzymes are identical to those of enzymes in solution (Schroen et al., 2002; van Roon et al., 2006). Nevertheless, immobilization of enzymes can affect their kinetic parameters, observed so far for immobilization techniques based on covalent binding (Berendsen et al., 2006; Buchholz, 1989). Up to date it is still unknown if immobilization of enzymes in hydrogel beads also alters their kinetic parameters.

To answer this question the model-based experimental analysis (MEXA) approach is adopted (Marquardt, 2005). As example, the stereoselective carboligation of two 3,5-dimethoxybenzaldehyde (DMBA) molecules to $(R)-3,3^{\prime}, 5,5^{\prime}$-tetramethoxybenzoin (TMB) using the enzyme benzaldehyde lyase (BAL, EC 4.1.2.28) is investigated. The enzymes are immobilized in $\kappa$-carrageenan hydrogel beads which are surrounded by methyl-iso-butylketone (MIBK) as organic solvent. In this gel-stabilized aqueous-organic biphasic system the phenomena enzyme reaction, mass transfer, and diffusion are superimposed. One approach is to lump all phenomena and to define a new set of pseudo-kinetic constants (Cho and Bailey, 1978; Schroen et al., 2002). However, the fitted constants are only valid for the reaction conditions the lumped model was derived for. Thus, such a lumped model is not suitable for the rational design of enzyme immobilizates (van Roon et al., 2006). In contrast to that 
approach, the MEXA approach aims at deriving a mechanistic kinetic model which accounts for all relevant phenomena in the system. Therefore, the individual kinetic models for each phenomenon (Chapters 2-4) are combined to one kinetic model. Then, the combined kinetic model is used to perform a priori simulations and a sensitivity analysis which supports the choice of an optimal measurement method. The kinetic model is fitted to highly resolved experimental data and the parameters are estimated. In the end, the estimated parameter values are compared to those for the individual systems (Chapters 2-4) and the combined systems (Chapters 5 and 6).

\subsection{Model development}

The modeling of enzyme immobilizates is typically performed by using empirical correlations and dimensionless numbers (Cho and Bailey, 1978; Magario et al., 2008; van Roon et al., 2006). According to Goncalves et al. (2002) mechanistic kinetic modeling is intractable. Despite these previous studies, an entirely mechanistic kinetic model considering mass transfer, diffusion, and enzyme reaction is developed here. Eqs. 7-1 and 7-2 are the mass balances for the bulk and the bead. $V_{b u l k}$ is the bulk volume, $t$ is the time, $r$ the radial position in the bead, $c_{i, b u l k}$ and $c_{i, \text { bead }}$ are the concentrations of the species $i$ in the bulk and in the bead, respectively. The parameter $k_{i}$ denotes the mass transfer coefficient, $A_{\text {bead }}$ the surface of the bead, $R$ the bead radius, $j_{i}$ the molar diffusive flux, $v_{\mathrm{i}}$ the stoichiometric coefficient, and $v$ the reaction rate.

$$
\begin{gathered}
V_{\text {bulk }} \frac{d c_{i, \text { bulk }}}{d t}=k_{i} \cdot A_{\text {bead }} \cdot\left(P_{i} \cdot c_{i, \text { bead }}(r=R)-c_{i, \text { bulk }}\right) \\
\frac{\partial c_{i, \text { bead }}}{\partial t}=-\frac{1}{r^{2}}\left(\frac{\partial}{\partial r} \cdot r^{2} \cdot j_{i}\right)+v_{i} \cdot v
\end{gathered}
$$

The diffusive flux can be modeled by using Fick's law (Chapters 4 and 6), where $D_{i, \text { eff }}$ is the effective diffusion coefficient taking the influence of the hydrogel material on the diffusion rate into account:

$$
j_{i}=-D_{i, e f f} \cdot \frac{\partial c_{i}}{\partial r}
$$


As demonstrated in Chapter 2, the reaction rate for the carboligation of two DMBA molecules to TMB using BAL can be expressed by Eq. 7-4, where $k_{\text {catf }}, K_{e q}, K_{m A}, K_{m B}, K_{m P}, K_{i A}$, and $K_{i B}$ are the enzyme kinetic parameters.

$$
V=\frac{\frac{K_{c a t f}}{K_{i A} \cdot K_{m B}} \cdot\left(c_{A, a q}{ }^{2}-\frac{c_{P, a q}}{K_{e q}}\right)}{1+\frac{c_{A, a q}}{K_{i A}}\left(1+\frac{K_{m A}}{K_{m B}}\right)+\frac{c_{A, a q}{ }^{2}}{K_{i A} \cdot K_{m B}}+\frac{c_{P}}{K_{m P}}+\frac{c_{A} \cdot c_{P}}{K_{m P} \cdot K_{i B}}} \cdot c_{E}
$$

$K_{i B}$ is a dependent model parameter and can be calculated using the independent parameters:

$$
K_{i B}=\frac{K_{m B} \cdot K_{i A}}{K_{m A} \cdot\left[1-\left(\frac{K_{m A}}{K_{i A}}-1\right) \cdot \frac{K_{m P}}{K_{e q} \cdot K_{m B} \cdot K_{i A}}\right]}
$$

As demonstrated in Chapter 2, the enzyme kinetic parameters are not identifiable unless a simplification is introduced. Thus, the model contains two additional equations, which reduces the number of independent model parameters to four $\left(k_{c a t}, K_{e q}, K_{m A}\right.$, and $\left.K_{m B}\right)$ ):

$$
\begin{gathered}
K_{m P}=\frac{K_{m B} \cdot\left(K_{m B}-K_{m A}\right)^{2} \cdot K_{e q}}{2 \cdot K_{m A}} \\
K_{i A}=K_{m B}-K_{m A}
\end{gathered}
$$

The kinetic model contains the same boundary and initial conditions as the kinetic model without reaction (Eqs. 6-4-6-7).

\subsection{A priori simulations}

In order to estimate the parameters for diffusion and enzyme reaction in the gel-stabilized system, it is important to avoid both the diffusion limited and the reaction limited regime. In the former case, the maximal reaction rate is much higher than the diffusion rate, whereas it is much lower in the latter case. According to Eq. 7-4, the reaction rate is directly proportional 
to the enzyme concentration. Thus, increasing the enzyme concentration is a straightforward method to avoid reaction limitation and vice versa for diffusion limitation. Therefore, a priori simulations with varying enzyme concentrations are carried out in order to determine an enzyme concentration avoiding both limitations. The a priori simulations are based on the kinetic model and on the nominal values for the model parameters (Table 7-1), which were determined in Chapters 2, 3, and 4.

Table 7-1: $\quad$ Nominal values for the model parameters previously determined in Chapters 2, 3 and 4.

\begin{tabular}{lll}
\hline parameter & nominal value & unit \\
\hline$k_{\text {catf }}$ & 40.37 & $\mathrm{~s}^{-1}$ \\
$K_{e q}$ & 1.464 & $\mathrm{mM}^{-1}$ \\
$K_{m A}$ & 0.147 & $\mathrm{mM}$ \\
$K_{m B}$ & 0.269 & $\mathrm{mM}$ \\
$P_{A}$ & 52.37 & - \\
$P_{P}$ & 84.36 & - \\
$D_{A, \text { eff }}$ & $4.139 \cdot 10^{-10}$ & $\mathrm{~m}^{2} \mathrm{~s}^{-1}$ \\
$D_{P, \text { eff }}$ & $2.261 \cdot 10^{-10}$ & $\mathrm{~m}^{2} \mathrm{~s}^{-1}$ \\
\hline
\end{tabular}

Figure 7-1 shows the substrate concentration in the hydrogel bead as a function of time and radius for three different enzyme concentrations. In all three cases the DMBA concentration in the hydrogel bead initially increases with time until a constant concentration is reached. The duration of this increase depends on the position in the bead. At the surface the concentration rapidly increases, whereas it lasts much longer in the bead center due to the higher diffusion distance. In the steady-state the rates of diffusion and reaction are equal. The thermodynamic equilibrium of the complete system is reached much later. Due to the low volume of the bead compared to the bulk volume $\left(\mathrm{V}_{\text {bulk }}: \mathrm{V}_{\text {bead }}=21.2: 1\right)$, the total amount of enzyme in the complete system is rather low. Thus, it can last days until the bulk concentrations reach a constant level. However, the concentrations in the bead do not change significantly after $2000 \mathrm{~s}$. Thus, experiments of this duration are sufficient. 

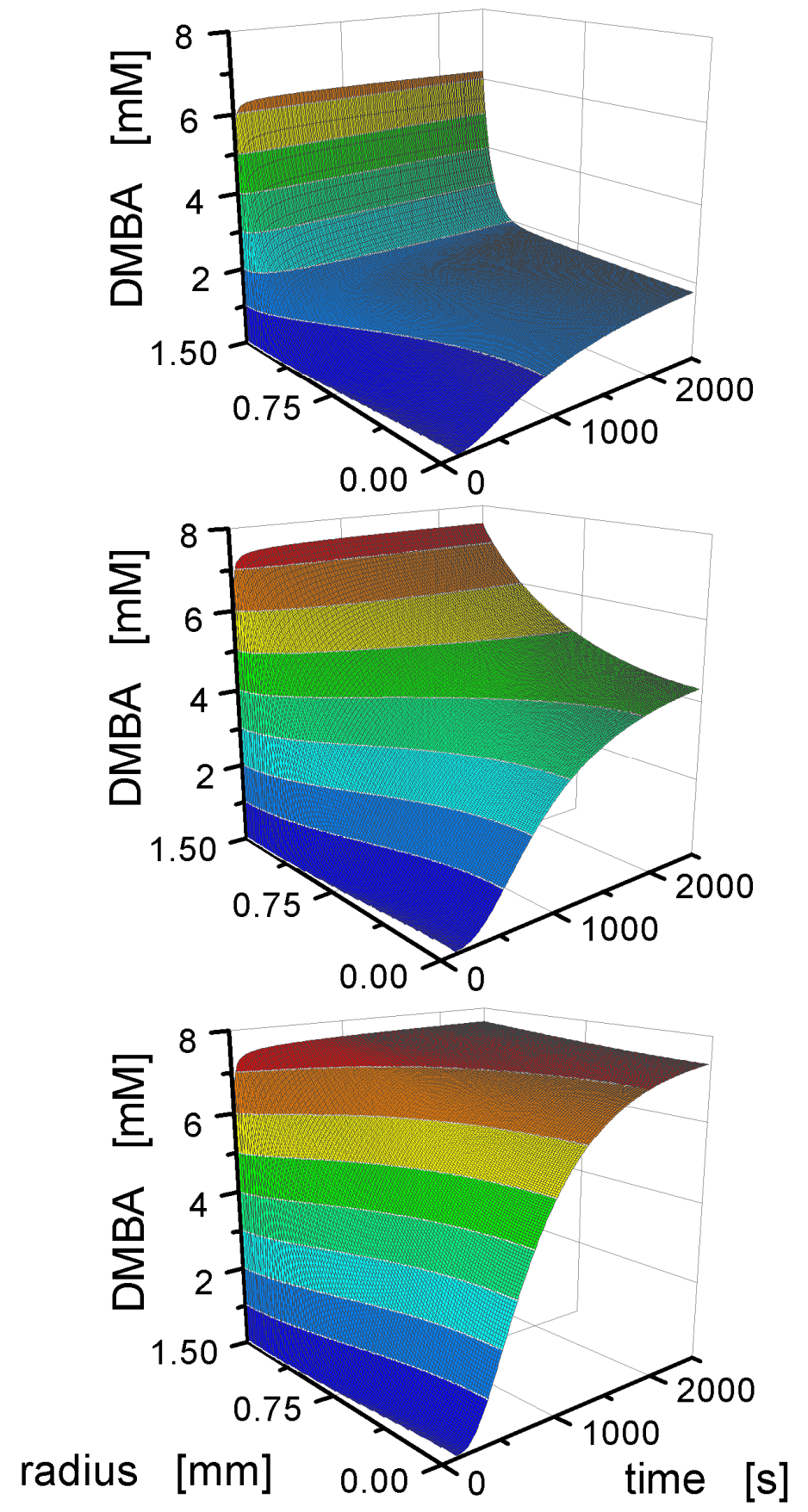

Figure 7-1: A priori simulations of the substrate concentration in the hydrogel bead using different enzyme concentrations (top: $3.34 \cdot 10^{-2} \mathrm{mM}$; middle: $3.34 \cdot 10^{-4} \mathrm{mM}$; bottom: $3.34 \cdot 10^{-6} \mathrm{mM}$ ) $\left(c_{A, b u l k, 0}=400 \mathrm{mM}, V_{\text {bulk }}=300 \mu \mathrm{L}, R=1.5 \mathrm{~mm}\right)$.

Moreover, the a priori simulations based on the nominal parameter values (Table 7-1) reveal that an enzyme concentration of $3.34 \cdot 10^{-2} \mathrm{mM}$ (which corresponds to $2 \mathrm{mg} \mathrm{mL}^{-1}$ ) causes diffusion limitation. In this case, the reaction takes place in a small zone close to the bead surface. The DMBA concentration reaches the equilibrium concentration of the reaction at approximately $75 \%$ of the bead radius and remains constant in the rest of the bead. Thus, a 
large part of the immobilized enzymes does not contribute to the reaction. Moreover, in this case measurements of the DMBA concentration in the bead center can only determine the thermodynamic equilibrium constant $K_{\text {eq }}$, but not the kinetic parameters. If the enzyme concentration is lowered, the reaction zone is broadened and reaches further into the bead. However, if the enzyme concentration is chosen too low, the system becomes reaction limited. At an enzyme concentration of $3.34 \cdot 10^{-6} \mathrm{mM}$ the radial concentration gradient is minimized with increasing time and the hydrogel bead approaches an ideally mixed system. Even in the bead center the DMBA concentration is far away from the equilibrium concentration of the reaction, thus, $K_{e q}$ is not identifiable. The concentration changes in the beginning are mostly caused by diffusion, whereas the enzyme reaction does not significantly affect the concentrations. In this case, only the effective diffusion coefficient of the substrate can be estimated. From Figure 7-1 it can be concluded that an enzyme concentration of $3.34 \cdot 10^{-4} \mathrm{mM}$ is in the optimal order of magnitude since here the reaction takes place over the whole radius resulting in significant radial concentration gradients.

Although these considerations aim at designing suitable experiments for parameter estimation, they apply also for the rational design of enzyme immobilizates. If the system is diffusion limited, most of the enzyme remains unused, lowering the productivity. For industrial application, one would not lower the enzyme concentration, but decrease the bead radius. However, this could cause other problems like increased pressure drop for a process carried out in a packed bed reactor.

\subsection{Sensitivity analysis}

The standard approach to investigate reaction systems with immobilized biocatalysts is to quantify the concentrations of the reactants by taking samples from the bulk phase. Typically these samples are analyzed by chromatographic methods like high performance liquid chromatography (HPLC) (Berendsen et al., 2006; Goncalves et al., 2002; 2008; Guisan et al., 1994; Magario et al., 2008; Schroen et al., 2002; van Roon et al., 2003; 2006). Moreover, capillary electrophoresis (Schroen et al., 2002), pH-titrimetry (Guisan et al., 1994), or enzyme-coupled methods like the glucose oxidase-peroxidase test are applied (Handrikova et al., 1996; Polakovic et al., 2001). To investigate if bulk measurements are suitable, this 
approach is compared with non-invasive concentration measurements in the bead center using a sensitivity analysis.

The sensitivity analysis is carried out using the nominal values of the kinetic parameters listed in Table 7-1. The normalized sensitivities of the parameters regarding the concentration of DMBA are calculated using Eq. 6-8 and approximated using finite differences. Figure 7-2 depicts the normalized sensitivities of the model parameters regarding the DMBA concentration in the bulk and in the bead center.
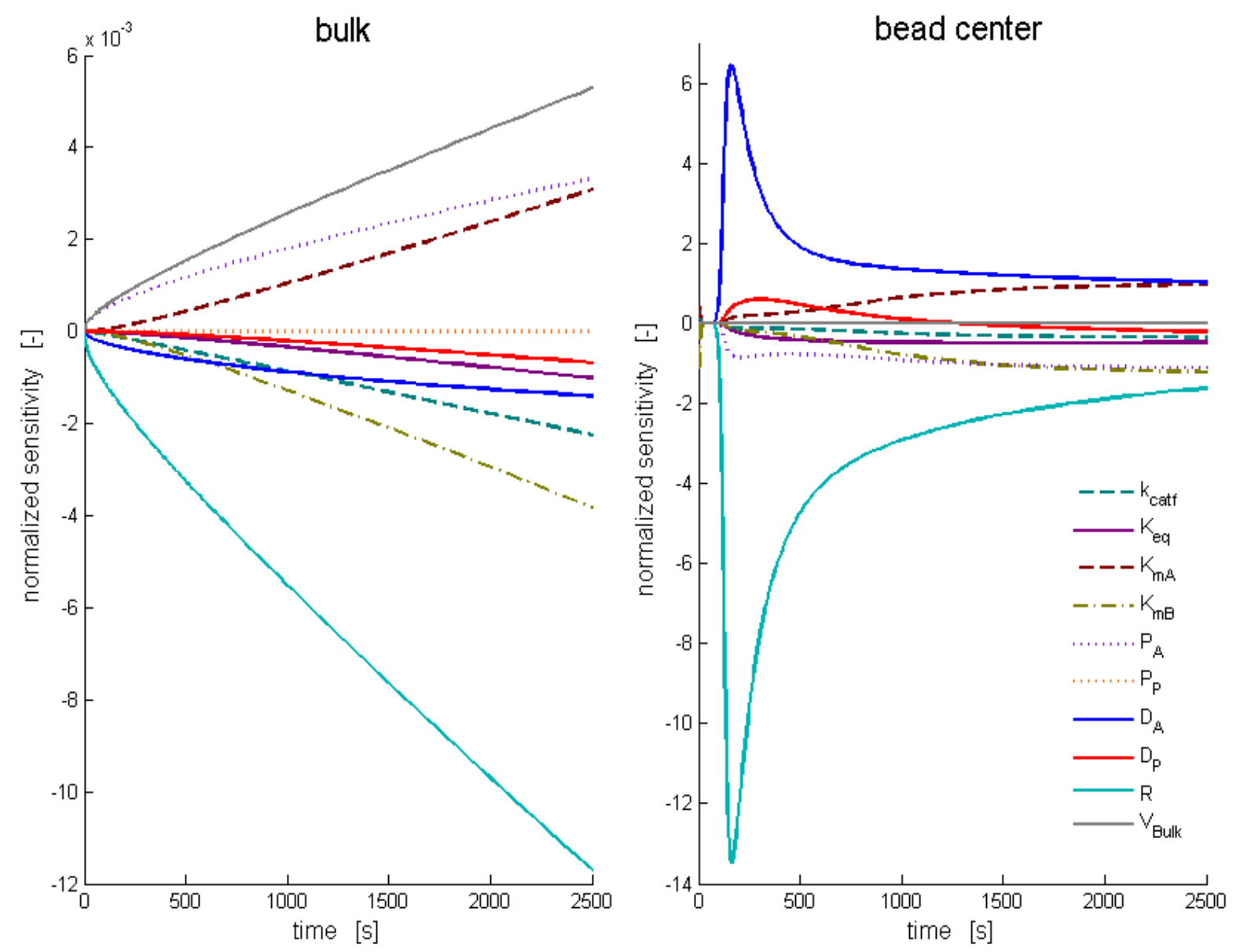

Figure 7-2: $\quad$ Normalized sensitivities of the model parameters regarding the DMBA concentration in the bulk phase (left) and in the bead center (right). The nominal parameter values listed in Table 7-1 were used. Based on the previous results it was assumed that the mass transfer at the interface is not rate-limiting $\left(c_{E}=3.34 \cdot 10^{-4} \mathrm{mM}, \quad c_{D M B A, b u l k, 0}=400 \mathrm{mM}, \quad V_{\text {bulk }}=300 \mu \mathrm{L}\right.$, $R=1.5 \mathrm{~mm})$.

According to Figure 7-2 (left) the sensitivities of the radius and of the bulk volume in the bulk phase are higher than all other model parameters. Thus, these two parameters significantly 
affect the measured bulk concentration of DMBA. If they are not known precisely, this hinders the exact determination of the other parameters. However, even if the radius and the bulk volume are precisely known, the values of the normalized sensitivities in the bulk are in all cases about three orders of magnitude lower than in the bead center. As already demonstrated in Chapters 4 and 6, measurements in the bead center are superior since the sensitivities of the model parameters are much higher in this case (Figure 7-2, right). In the bead center the bulk volume is not sensitive. Thus, inaccurate pipetting of the organic solvent does not influence the parameter estimates. However, the radius still exhibits the highest normalized sensitivity. For this reason, the radius has to be determined separately.

Besides the radius the effective diffusion coefficient of DMBA is the most sensitive parameter in the bead center in the beginning. As already observed in Chapter 4.2.3, the sensitivity maximum is reached when the diffusion front reaches the bead center. However, the normalized sensitivity does not approach zero as it was the case in Chapter 4.2.3. In contrast to this, the effective diffusion coefficient remains sensitive for longer times. The reason for this is that steady-state is reached where the diffusion rate and the reaction rate are equal. The normalized sensitivities of the enzyme kinetic constants increase by time and approach the same order of magnitude as the effective diffusion coefficient of DMBA in the steady-state.

The a priori simulations revealed that radial concentration gradients are formed (Section 7.3). Thus, the normalized sensitivities depend also on the radial position in the hydrogel bead. Figure 7-3 shows the normalized sensitivity of the parameter $K_{m A}$ as example.

According to Figure 7-3 the maximum of the normalized sensitivity of a parameter is not necessarily located in the bead center. Since the sensitivity maxima of the model parameters lie at different radial positions, measurements along the whole radius should be preferred over measurements only in the bead center. Hence, information is also collected about the parameters which are sensitive in the outer shells of the bead, and thus the model parameters can be estimated more precisely. 


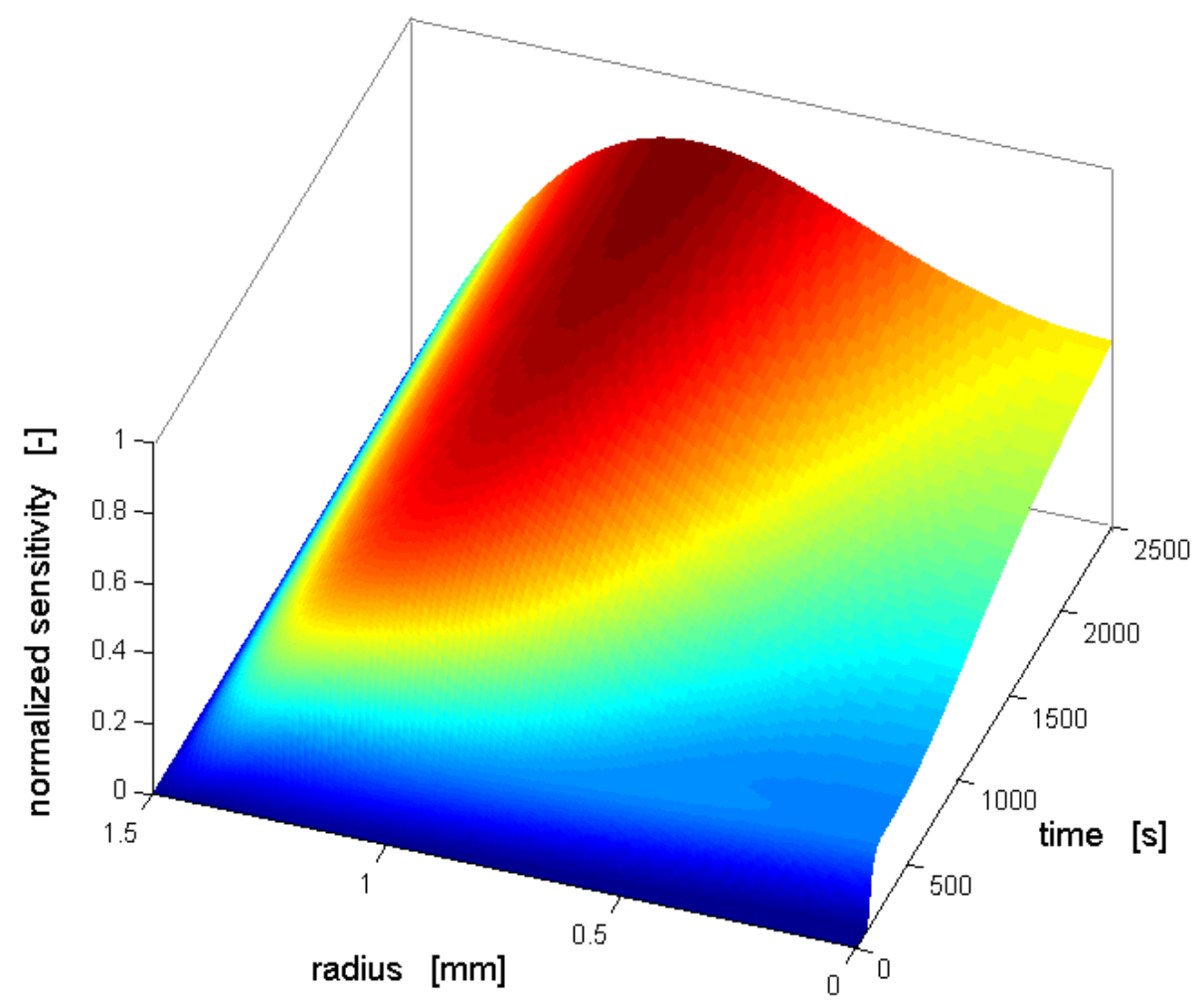

Figure 7-3: Normalized sensitivity regarding the DMBA concentration of the parameter $K_{m A}$ as a function of time and radius. The nominal parameter values listed in Table 7-1 were used $\left(c_{E}=6.69 \cdot 10^{-4} \mathrm{mM}, c_{A, b u l k, 0}=400 \mathrm{mM}, V_{\text {bulk }}=300 \mu \mathrm{L}, R=1.5 \mathrm{~mm}\right)$.

\subsection{Materials and methods}

The same chemicals as in the previous chapters were used. $\kappa$-carrageenan hydrogel beads were produced by adding $1.5 \%(\mathrm{w} / \mathrm{v})$ carrageenan to $50 \mathrm{mM} \mathrm{KH} \mathrm{KO}_{4} / \mathrm{K}_{2} \mathrm{HPO}_{4}$ buffer. The $\mathrm{pH}$ value was adjusted to 7.75 and the ionic strength to $100 \mathrm{mM}$. The suspension was heated up to $100^{\circ} \mathrm{C}$ to dissolve the carrageenan. Subsequently, the solution was cooled down to $45^{\circ} \mathrm{C}$. At this temperature it remains in the liquid state. Then the enzyme as well as the cofactors $\mathrm{MgSO}_{4}$ and thiamin diphosphate (ThDP) were added. The final concentrations were $20 \mu \mathrm{g} \mathrm{mL}^{-1}\left(3.34 \cdot 10^{-4} \mathrm{mM}\right)$ for BAL and $0.25 \mathrm{mM}$ for the cofactors. Higher temperatures were avoided in order to minimize heat denaturation of the enzymes. Using a pipette with a temperature jacket $20 \mu \mathrm{L}$ of the solution were dropped into a column containing vegetable oil. The column was heated at the top and cooled at the bottom resulting in a temperature gradient from $45^{\circ} \mathrm{C}$ to $0^{\circ} \mathrm{C}$. Due to the minimization of the interface between oil and aqueous solution round drops were obtained which harden to hydrogel beads by cryogelation. The beads were stored at $4{ }^{\circ} \mathrm{C}$ until experimental usage. Since DMF reduces the storage stability of BAL 
(Schmidt, 2008), the beads were equilibrated with a water-saturated MIBK solution containing 2.56\%(v/v) DMF just before the start of the experiment. Thus, DMF diffuses into the bead resulting in an equilibrium volume fraction of $25 \%(\mathrm{v} / \mathrm{v})$ increasing the solubility of the reactants DMBA and TMB and raising the $\mathrm{pH}$ value to 8.5 .

The experiments were performed in a 96-well microtiter plate at room temperature. The hydrogel bead was placed on top of a circular holder to guarantee uniform conditions. The experiments were started by the addition of $300 \mu \mathrm{L}$ of methyl-iso-butyl-ketone (MIBK) containing 300 or $400 \mathrm{mM}$ DMBA and $2.56 \%(\mathrm{v} / \mathrm{v})$ DMF. Five experiments were conducted with an initial concentration of $300 \mathrm{mM}$ DMBA, seven experiments with $400 \mathrm{mM}$ DMBA as initial concentration. To avoid evaporation of MIBK, the well of the microtiter plate was sealed.

As demonstrated in the previous Section, concentration measurements at different radial positions in the hydrogel bead are the optimal measurement method. This can be achieved by line scans using two-photon confocal laser scanning microscopy (CLSM). Details concerning this device can be found in Chapter 4.2.4. For excitation of DMBA a wavelength of $723 \mathrm{~nm}$ with a power of $42 \mathrm{~mW}$ was used. Fluorescence of TMB was neglected. Calibration was performed by using hydrogel beads which were equilibrated with concentrations of 0,100 , 200, 300, and $400 \mathrm{mM}$ DMBA. For each concentration 10 beads were used. Using the measured fluorescence intensities, linear calibration curves were obtained.

For each of the 12 experiments the concentration was measured at 64 positions and at 320 time points, resulting in 20,480 data points per single experiment. Some of the 64 positions were located beyond the interface depending on the actual bead size. The interface was detected by an unsteadiness of the experimental data. In order to reduce the computational load for parameter estimation and to reduce noise, the experimental data matrix was smoothed using smoothing splines (de Boor, 1978; Reinsch, 1967). From the smoothed matrix 8 radial positions and 50 time points were extracted. Thus, the data amount was reduced from 20,480 to 400 data points per experiment.

After each measurement, the correct position and the quality of the hydrogel bead were controlled using light microscopy. If they were not satisfactory, the respective data were not 
considered. According to the results of the sensitivity analysis, the bead radius was measured using the method of Buthe et al. (2004).

\subsection{Results and discussion}

The combined kinetic model was fitted to 12 experiments in order to estimate all model parameters for mass transfer, diffusion, and enzyme reaction simultaneously. This is in contrast to the approach of other research groups. In some studies the model parameters are estimated on the basis of experiments that focus on the individual phenomena only. The values of the parameters are assumed to be independent from the reaction system and then the model prediction is only qualitatively compared with experimental results for the complete system (van Roon et al., 2006). In other studies only parts of the model parameters are estimated using the complete system, whereas other parameters are obtained separately. For example, Goncalves et al. (2008) only estimate the effective diffusion coefficients in the complete system, whereas the enzyme kinetic parameters are taken from experiments with enzymes in solution. Conversely, Berendsen et al. (2006) estimate the enzyme kinetic parameters in the complete system, while the effective diffusion coefficients are calculated using empirical correlations.

An iterative approach was applied for parameter estimation. In a first step, the bead radius, the enzyme concentration and the lag-time were individually estimated for each experiment. The optically determined Feret diameter was used for the calculation of the initial guess of the bead radius. For the enzyme concentration the nominal concentration within the carrageenan solution was used. Since the correct position of the bead on the circular holder had to be verified after addition of the bulk phase, the lag-time, i.e. the duration between the start of the experiment and the start of the measurement, deviates from bead to bead. Thus, the lag-time was also individually estimated for each bead. The other parameters were set to their nominal values (Table 7-1). In the next step, the radius, the enzyme concentration and the lag-time were fixed and the other parameters estimated using the nominal values as initial guesses. These two steps were repeated four times using the estimates of the preceding steps as initial guesses. Figure 7-4 shows the fit of the model to experimental data. As example, four experiments are selected. 

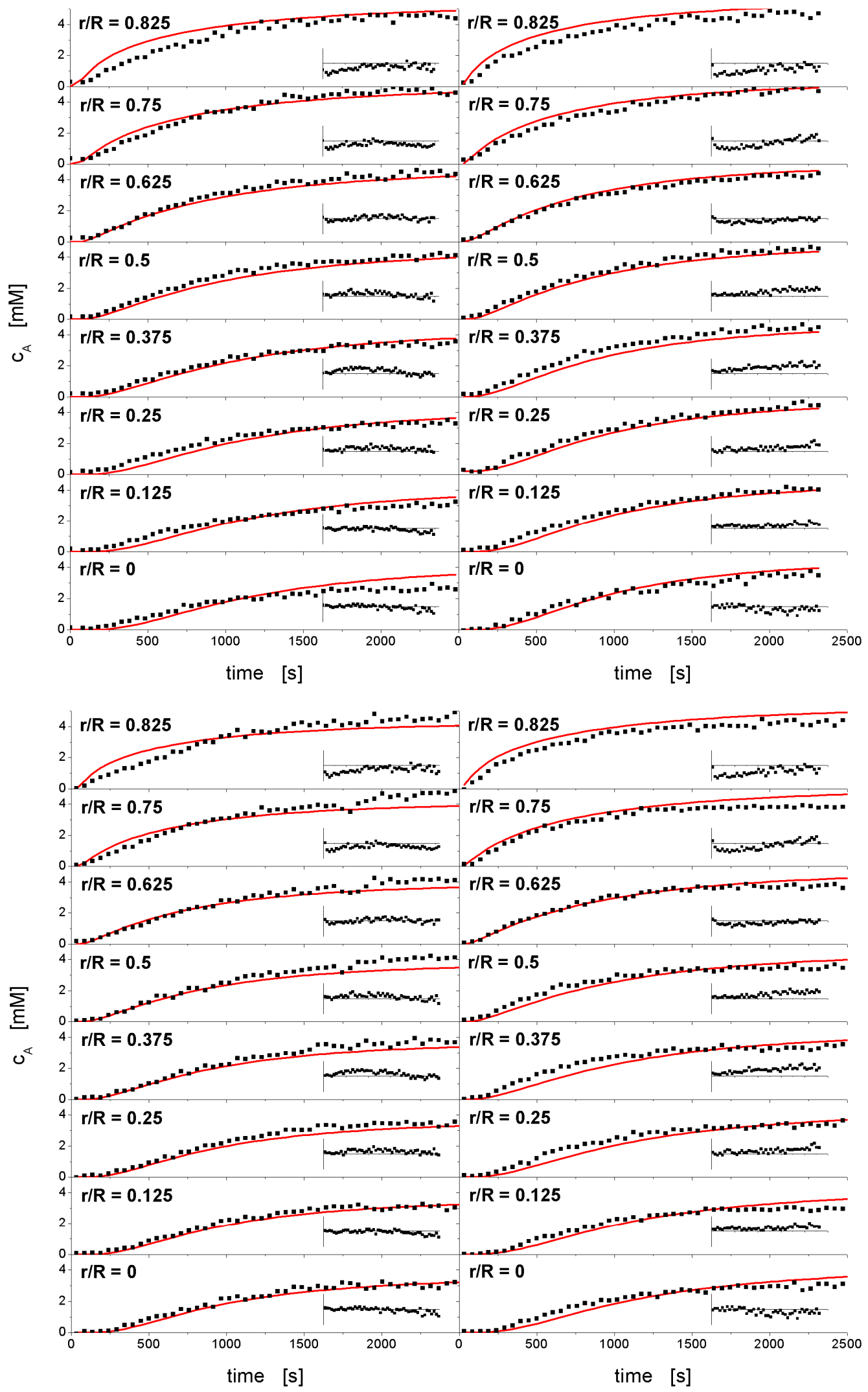

Figure 7-4: $\quad$ Fit of the kinetic model to experimental data at 8 different radial positions. 4 of 12 experiments are shown as example. The residuals are shown as small inlets. The experimental conditions are: $c_{A, \text { bulk }, 0}=400 \mathrm{mM}, \quad c_{P, \text { bulk }, 0}=0 \mathrm{mM}, \quad c_{A, \text { bead }, 0}=0 \mathrm{mM}, \quad c_{P, b u l k, 0}=0 \mathrm{mM}$, $c_{E}=3.34 \cdot 10^{-4} \mathrm{mM}, V_{\text {bulk }}=300 \mu \mathrm{L}, R=1.5 \mathrm{~mm}$. 
Obviously, the kinetic model is able to satisfactorily describe the experimental data at all radial positions. Only close to the interface the residuals indicate minor systematic deviations. One reason could be the difficulty in identifying the correct position of the interface from the experimental data (Section 5.1.5). A higher enzyme concentration close to the surface could also explain why the measured substrate concentrations close to the interface are lower than those predicted by the kinetic model. Higher enzyme concentrations close to the interface were observed for gelatin-chitosan particles (Assemblase ${ }^{\circledR}$ ) (van Roon et al., 2005) or for alginate hydrogel beads at low protein loadings (Heinemann, 2003). Therefore, the assumption of a homogeneous distribution of the enzymes within the $\kappa$-carrageenan hydrogel bead should be investigated in further studies. This can be achieved by several labeling and detection techniques (van Roon et al., 2002; 2005).

Table 7-2: $\quad$ Obtained parameter estimates and their standard deviations.

\begin{tabular}{llll}
\hline parameter & estimate & standard deviation & unit \\
\hline$k_{\text {catf }}$ & 45.27 & 21.07 & $\mathrm{~s}^{-1}$ \\
$K_{\text {eq }}$ & 1.418 & 0.175 & $\mathrm{mM}^{-1}$ \\
$K_{m A}$ & 0.147 & 0.117 & $\mathrm{mM}$ \\
$K_{m B}$ & 0.269 & 0.141 & $\mathrm{mM}$ \\
$k_{A}$ & $1.90 \cdot 10^{-08}$ & $8.25 \cdot 10^{-10}$ & $\mathrm{~m} \mathrm{~s}^{-1}$ \\
$k_{P}$ & $7.30 \cdot 10^{-09}$ & $1.07 \cdot 10^{-6}$ & $\mathrm{~m} \mathrm{~s}^{-1}$ \\
$P_{A}$ & 53.46 & 1.34 & - \\
$P_{P}$ & 72.06 & $1.05 \cdot 10^{4}$ & - \\
$D_{A, \text { eff }}$ & $4.35 \cdot 10^{-10}$ & $1.57 \cdot 10^{-11}$ & $\mathrm{~m}^{2} \mathrm{~s}^{-1}$ \\
$D_{P, \text { eff }}$ & $1.60 \cdot 10^{-10}$ & $1.92 \cdot 10^{-11}$ & $\mathrm{~m}^{2} \mathrm{~s}^{-1}$ \\
\hline
\end{tabular}

The obtained parameter estimates are listed in Table 7-2. In contrast to other studies on immobilized enzymes (e.g. Berendsen et al., 2006; Spiess et al., 1999; van Roon et al., 2006) also standard deviations of the kinetic parameters are presented. Table 7-2 reveals that the precision of the obtained experiments is quite diverse. $K_{e q}, k_{A}, P_{A}$, and $D_{A, \text { eff }}$ possess relatively low standard deviations. The precision of the parameters $k_{\text {catf }}$ and $D_{P, \text { eff }}$ is considerably lower. The parameters $K_{m A}, K_{m B}, k_{P}$, and $P_{P}$ are not identifiable under the applied conditions. The unidentifiability of these four parameters can also be seen in the correlation matrix (Table 7-3). High correlations exist between $K_{m A}$ and $K_{m B}$ and between $k_{P}$ and $P_{P}$. The former was 
already observed in Chapter 2 and is caused by similar definitions of $K_{m A}$ and $K_{m B}$ in terms of micro-reaction rate constants. The latter is probably due to the fact that the product concentration cannot be quantified.

Table 7-3: Correlation matrix of the parameter estimates.

\begin{tabular}{|c|c|c|c|c|c|c|c|c|c|c|}
\hline parameter & $k_{\text {catf }}$ & $K_{\text {eq }}$ & $K_{m A}$ & $K_{m B}$ & $k_{A}$ & $k_{P}$ & $P_{A}$ & $P_{P}$ & $D_{A, \text { eff }}$ & $D_{P, \text { eff }}$ \\
\hline$k_{\text {catf }}$ & 1.00 & 0.41 & 0.58 & 0.46 & 0.82 & -0.19 & -0.94 & 0.19 & 0.78 & -0.67 \\
\hline$K_{e q}$ & & 1.00 & 0.75 & 0.72 & 0.09 & -0.12 & -0.23 & 0.12 & 0.07 & 0.23 \\
\hline$K_{m A}$ & & & 1.00 & 0.99 & 0.04 & 0.02 & -0.54 & -0.02 & 0.01 & -0.08 \\
\hline$K_{m B}$ & & & & 1.00 & -0.09 & 0.05 & -0.43 & -0.05 & -0.12 & 0.01 \\
\hline$k_{A}$ & & & & & 1.00 & -0.28 & -0.72 & 0.28 & 0.96 & -0.74 \\
\hline$k_{P}$ & & & & & & 1.00 & 0.07 & -1.00 & -0.36 & 0.15 \\
\hline$P_{A}$ & & & & & & & 1.00 & -0.08 & -0.70 & 0.70 \\
\hline$P_{P}$ & & & & & & & & 1.00 & 0.36 & -0.15 \\
\hline$D_{A, \text { eff }}$ & & & & & & & & & 1.00 & -0.74 \\
\hline$D_{P, \text { eff }}$ & & & & & & & & & & 1.00 \\
\hline
\end{tabular}

In contrast to the assumption used for the a priori simulations and the sensitivity analysis, the obtained parameter estimates indicate a mass transfer limitation at the interface. In Chapters 4 and 6 no such limitation was observed. A possible explanation could be that due to the superposition of diffusion and enzyme reaction, the substrate decrease close to the interface is enhanced. Therefore, mass transfer becomes limiting, whereas in a system without enzyme the diffusive flux alone is slower than the mass transfer flux. However, in the gel-stabilized aqueous-organic biphasic reaction system many other factors may affect the obtained parameter estimates. Among them are the difficulty in identifying the position of the interface, a higher enzyme concentration close to the interface, a possible heat denaturation of enzymes during bead production, and the deviation from the ideally spherical geometry of the hydrogel beads. Moreover, it is possible that the laser light causes a slight temperature increase in the bead affecting the kinetic processes. Furthermore, the simultaneous estimation of ten model parameters may result in local instead of global optima (Marquardt, 2005). 


\subsection{Comparison of parameter estimates}

In this Section it is investigated if the coupling of the phenomena enzyme reaction, mass transfer, and diffusion affects the values of the obtained parameter estimates. If this is not the case, it would be sufficient to determine the model parameters using the individual systems and then to use these values for the combined system. This would facilitate the optimization of such gel-stabilized aqueous-organic biphasic reactive systems. However, the phenomena may interact with each other, which could result in different values of the model parameters. For this reason, the obtained parameter estimates for the complete system are compared with those obtained for the individual and coupled systems. However, the problems in estimating the model parameters for the final system should be kept in mind. First the enzyme kinetic parameters are compared (Table 7-4).

Table 7-4: $\quad$ Comparison of the parameter estimates and their standard deviations for the enzyme reaction. The values for the individual system were determined in Chapter 2.5, those for the coupling of enzyme reaction and mass transfer in Chapter 5.1.

\begin{tabular}{|c|c|c|c|c|}
\hline & $k_{\text {catf }}$ & $K_{e q}$ & $K_{m A}$ & $K_{m B}$ \\
\hline unit & $s^{-1}$ & $\mathrm{mM}^{-1}$ & $\mathrm{mM}$ & $\mathrm{mM}$ \\
\hline enzyme kinetics & $40.37 \pm 0.98$ & $1.464 \pm 0.008$ & $0.147 \pm 0.009$ & $0.269 \pm 0.014$ \\
\hline $\begin{array}{l}\text { enzyme kinetics } \\
\text { and mass transfer }\end{array}$ & $42.51 \pm 18.17$ & $1.577 \pm 0.554$ & $0.136 \pm 0.109$ & $0.251 \pm 0.141$ \\
\hline combined system & $45.27 \pm 21.07$ & $1.418 \pm 0.175$ & $0.147 \pm 0.117$ & $0.269 \pm 0.141$ \\
\hline
\end{tabular}

According to Table 7-4 the optimal estimates for all three systems are close to each other. However, the standard deviations strongly depend on the system. In general, the lowest standard deviations are obtained in the individual system - the enzyme reaction in a homogeneous aqueous system (Chapter 2.5). The standard deviations in the coupled and combined system are in the same range, although in the combined system diffusion has to be considered additionally. There are two main reasons: First the CLSM measurement technique in the combined system turns out to be more precise and reliable than the fluorescence and extinction measurements applied for the coupled system in the Lewis cell. Second in the 
combined system temporal and radial concentration profiles are obtained. Thus, more data with different substrate concentrations are obtained. Taking the standard deviations into account, no statistically significant influence of the system on the parameter estimates can be identified. Neither a detrimental effect due to the presence of the organic solvent, nor a change of enzyme activity due to immobilization in hydrogel beads can be deduced.

Table 7-5: $\quad$ Comparison of the parameter estimates and their standard deviations for the mass transfer. The values for the individual system were determined in Chapter 3, those for the coupling of enzyme reaction and mass transfer in Chapter 5.1. Due to different geometries the values of the mass transfer coefficients from the individual system cannot be compared to those using hydrogel beads.

\begin{tabular}{lllll}
\hline & \multicolumn{1}{l}{$k_{A}$} & $k_{P}$ & $P_{A}$ & $P_{P}$ \\
\hline unit & $\mathrm{m} \mathrm{s}^{-1}$ & $\mathrm{~m} \mathrm{~s}^{-1}$ & - & - \\
\hline \multirow{2}{*}{ mass transfer } & $5.39 \cdot 10^{-7}$ & $2.81 \cdot 10^{-7}$ & 52.37 & 84.36 \\
& $\pm 1.33 \cdot 10^{-9}$ & $\pm 3.33 \cdot 10^{-9}$ & \pm 0.13 & \pm 0.99 \\
& & & 52.76 & 82.25 \\
enzyme kinetics & not identifiable & not identifiable & \pm 0.17 & \pm 0.30 \\
and mass transfer & & & & 72.06 \\
& & & 53.46 & $\pm 1.05 \cdot 10^{4}$ \\
\hline
\end{tabular}

Table 7-5 lists the parameter estimates and their standard deviations for the mass transfer coefficients and the partition coefficients. The mass transfer coefficients for the individual system and for the complete system cannot be expected to be identical. In the individual system the two phases are stirred and the interface is a flat horizontal plane while in the complete system the aqueous phase is a spherical hydrogel bead. The ratio of the estimates of the mass transfer coefficients of substrate and product seems to be similar. However, the mass transfer coefficient of the product in the combined system is not identifiable. Since the experiments for the coupled system were carried out in the reaction limited regime, the mass transfer coefficients in this system are not identifiable (Chapter 5.1).

The partition coefficient of the substrate can be precisely determined in each system and the estimated values match very well. Obviously, the presence of enzyme or hydrogel does not 
considerably affect the substrate partition between both phases. The estimate of the product partition coefficient in the coupled system (Chapter 5.1) is consistent with the one of the individual system (Chapter 3). In the combined system it is, however, not identifiable. In summary, the values for the partition coefficients determined using the individual system can also be used for the coupled and combined system. In contrast to this, the mass transfer coefficients have to be estimated separately for each system.

Table 7-6: Comparison of the parameter estimates and their standard deviations for the effective diffusion coefficients. The values for the individual system were determined in Chapter 4.2, those for the coupling of diffusion and mass transfer in Chapter 6.

\begin{tabular}{lll}
\hline & $D_{A, \text { eff }}$ & $D_{P, \text { eff }}$ \\
\hline unit & $\mathrm{m}^{2} \mathrm{~s}^{-1}$ & $\mathrm{~m}^{2} \mathrm{~s}^{-1}$ \\
\hline $\begin{array}{l}\text { diffusion } \\
\text { diffusion } \\
\text { and mass transfer }\end{array}$ & $4.14 \cdot 10^{-10} \pm 3.88 \cdot 10^{-13}$ & $2.26 \cdot 10^{-10} \pm 6.20 \cdot 10^{-13}$ \\
& $4.25 \cdot 10^{-10} \pm 2.86 \cdot 10^{-13}$ & $3.11 \cdot 10^{-10} \pm 7.78 \cdot 10^{-13}$ \\
combined system & $4.35 \cdot 10^{-10} \pm 1.57 \cdot 10^{-11}$ & $1.60 \cdot 10^{-10} \pm 1.92 \cdot 10^{-11}$ \\
\hline
\end{tabular}

In Table 7-6 the effective diffusion coefficients for the substrate and the product in the three gel-stabilized systems are being compared. The estimates for the effective diffusion coefficient of the substrate are almost identical in all three systems and are also in line with the result obtained using nuclear magnetic resonance (NMR) spectroscopy in a cylindrical hydrogel column and $n$-hexane as organic bulk phase $\left(4.03 \cdot 10^{-10} \pm 5.94 \cdot 10^{-12}\right.$ - Chapter 6.7). Conversely, the estimates for the product differ from system to system. The standard deviations in the individual and in the coupled system are very low, while they are more than one order of magnitude higher in the combined system. However, they are still lower than those published by Polakovic et al. (2001), who obtained standard deviations for the effective diffusion coefficients of up to $28 \%$. From these results it can be concluded that the effect of the organic solvent or the enzyme on the diffusion rate of the substrate is rather low, whereas the product diffusion seems to depend on the system. As mentioned in the previous chapters, the product can easily precipitate. Moreover, its quantum yield and purity is lower compared 
to the commercially available substrate. Thus, the estimates for the product are less reliable than those for the substrate.

\subsection{Conclusions}

On the basis of the kinetic models for the individual and coupled systems, a kinetic model for the gel-stabilized aqueous-organic biphasic reactive system was derived in this chapter. In order to estimate all kinetic parameters concurrently, it is essential to avoid limitations due to diffusion or reaction. Using a priori simulations a suitable enzyme concentration was determined avoiding both limitations. A sensitivity analysis of the model parameters revealed that the optimal measurement method for estimating the model parameters is the quantification of the concentration along the radius of the hydrogel bead. These line-scans were achieved by two photon LSM. The derived kinetic model was in agreement with the experimental data and most model parameters could be determined satisfactorily. Only the Michalis-Menten constants, the mass transfer coefficient of the product, and the partition coefficient of the product could not be identified under the applied conditions.

The comparison of the parameter estimated with those from the individual and coupled systems indicates that no significant influence of the reaction system on the enzyme kinetic parameters can be observed. Moreover, no considerable influence on the partition coefficients and on the effective diffusion coefficient of the substrate was detected. However, the mass transfer coefficients and the effective diffusion coefficient of the product deviate from system to system, which makes it necessary to determine these parameters separately for each system. Thus, the widespread assumption that the model parameters obtained for the individual systems can also be used for the complete system, is not justifiable in all cases.

The presented experiments are the first ones which apply non-invasive optical measurement techniques for the quantification of enzyme kinetics in hydrogel beads. Certainly, the precision of the model parameters can further be increased by refining the measurement and analysis techniques. In particular, three aspects should be investigated in future studies on the complete system. First the enzyme distribution should be determined using e.g. fluorescent labeling. Second improvements for the identification of the interface position are desirable. Third a possible temperature increase caused by laser light should be quantified. If it turns out 
to be significant, efforts should be made in order to minimize this effect or to consider this in the kinetic model. Since immobilization often improves the long-term stability of enzymes, it should be verified if this is also true for BAL in $\kappa$-carrageenan hydrogel beads. 


\section{Conclusions and Outlook}

Aqueous-organic biphasic systems are important reaction systems for many catalytic processes and facilitate the biocatalytic production of hydrophobic fine chemicals. However, organic solvents can interact with the biocatalyst, thereby often reducing its catalytic activity. Therefore, immobilization of the biocatalyst in hydrogel beads suspended in organic solvents is a promising approach. As an example, the stereoselective carboligation of two 3,5dimethoxy-benzaldehyde (DMBA) molecules to $(R)$-3,3',5,5'-tetramethoxy-benzoin (TMB) using the enzyme benzaldehyde lyase (BAL, EC 4.1.2.38) from Pseudomonas fluorescens was investigated. BAL was immobilized in $\kappa$-carrageenan hydrogel beads which were surrounded by an organic solvent.

In the investigated gel-stabilized aqueous-organic biphasic reaction system a superposition of three kinetic phenomena occurs - enzyme reaction, mass transfer between the aqueous and the organic phase, and diffusion in the hydrogel beads. After studying these three phenomena separately, the individual systems were successively coupled. In the end the individual systems were combined to form the gel-stabilized aqueous-organic biphasic reaction system. Comparing the results enabled the detection of interactions between these phenomena. In order to systematically investigate each of the systems, the model-based experimental analysis (MEXA) approach was applied. Mechanistic kinetic models were derived which were subsequently used to perform a priori simulations and sensitivity analyses. This supports the determination of optimal measurement methods and the development of new experimental setups. Optimal experiments were designed and highly resolved measurement techniques applied in order to estimate the model parameters with high precision. As a result, limitations and bottlenecks in the catalytic mechanism were identified.

The first individual system investigated was the enzyme reaction in a homogeneous aqueous phase (Chapter 2). To emphasize how important the choice of an appropriate analysis program can be for the estimation of enzyme kinetic parameters, five analysis programs were compared concerning their reliability and precision. It could be demonstrated that large discrepancies in parameter estimates can occur, which should contribute to raise scientists' awareness of this problem. Afterwards, the first mechanistic kinetic model for the symmetric carboligation using BAL was derived. Since the model allows the calculation of the micro- 
reaction rate constants, conclusions about the enzyme mechanism could be drawn. The release of the product was identified to be the rate-limiting step in the enzymatic mechanism of BAL. In the next step, the mechanistic kinetic model was also applied to model the symmetric carboligation using benzoylformate decarboxylase (BFD). Although BAL and BFD are both thiamine diphosphate dependent enzymes, the catalytic bottleneck for BFD is not the product release but the substrate binding. A mechanistic explanation of this discrepancy is based on different active site geometries. The existence of different bottlenecks for BAL and BFD was verified by steady-state intermediate analysis using ${ }^{1} \mathrm{H}$ nuclear magnetic resonance. Furthermore, in Chapter 2 the modeling of enzyme kinetics using micro-reaction rate constants instead of macroscopic model parameters was demonstrated. While this approach has no benefit concerning parameter precision, it facilitates mechanistic conclusions. Moreover, general guidelines for the design of optimal experiments were formulated for symmetric carboligation reactions.

In Chapter 3 the mass transfer between an aqueous and an organic phase was investigated. To achieve high extractable yields, a well-suited organic solvent should exhibit a high ratio of the partition coefficients for the product and the substrate. For the purpose of screening a large amount of solvents, the screening was carried out ab initio using the Conductor-like Screening Model for Realistic Solvation (COSMO-RS). The ranking of solvent candidates according to the ratio of the partition coefficients indicated that methyl-iso-butyl-ketone (MIBK) is a suitable organic solvent for the investigated carboligation. Experiments with either MIBK or $n$-hexane as organic solvent were conducted and used for fitting the derived kinetic model for the mass transfer. The obtained estimates for the partition coefficients verified that MIBK is more suitable than $n$-hexane for achieving high extractable yields.

The diffusion in hydrogel beads was studied in Chapter 4. A sensitivity analysis demonstrated that the optimal method for investigating the diffusion in hydrogel beads is to measure in the bead center, since the sensitivities of the effective diffusion coefficients are then orders of magnitudes higher than in the bulk. This is in contrast to the standard procedure applied by other research groups, who usually perform only bulk measurements. First the diffusion of the dissociating species propionic acid was analyzed using fluorescence lifetime confocal laser scanning microscopy (CLSM). Two diffusion laws were compared, Fick's law and NernstPlanck law. It was found out that if an aqueous bulk phase is used, only Nernst-Planck law is able to describe the observed $\mathrm{pH}$ decrease in alginate hydrogel beads correctly, whereas in the 
presence of an organic bulk phase no model discrimination is possible. No significant influence of the alginate matrix on the diffusion rate of propionic acid could be detected. Furthermore, the effective diffusion coefficients of DMBA and TMB were precisely determined using $\kappa$-carrageenan hydrogel beads and two photon LSM. Finally, ${ }^{1} \mathrm{H}$ NMR diffusometry was carried out in order to study the diffusion of DMBA in $\kappa$-carrageenan hydrogel cylinders. Due to the longer duration of the NMR experiments, swelling of the hydrogel occurred, thus influencing the results.

In Chapter 5 the phenomena enzyme reaction and mass transfer were coupled. Due to their superposition, measurements only in the aqueous phase are not sufficient to quantify both phenomena. Thus, additional measurements in the organic phase are required enabling the quantification of mass transfer separately. It was furthermore demonstrated that the enzyme kinetic parameters can only be identified if the reaction is rate-limiting. Two approaches to achieve this goal were pursued. One possibility is to conduct the experiment in a Lewis cell with two distinct phases. In this case, reaction limitation can be achieved by decreasing the enzyme concentration, which causes long durations of the experiments. Although the derived kinetic model described the experimental data satisfactorily, the enzyme kinetic parameters could not be estimated with sufficient precision. However, improvement of the measurement methods in terms of accuracy and reliability might enhance the parameter precision in future studies. The alternative possibility to achieve reaction limited conditions is to increase mass transfer by emulsification. One drawback of reactive emulsions is the difficulty in applying non-invasive measurement techniques. Moreover, aggregates were observed which indicates that the increased interfacial area causes enzyme precipitation due to phase toxicity.

The coupling of diffusion and mass transfer was studied by two photon LSM measurements in the center of hydrogel beads which were surrounded by the organic solvent MIBK (Chapter 6). The obtained diffusion coefficient of DMBA is in line with the one obtained using an aqueous bulk and also with the one determined by ${ }^{1} \mathrm{H}$ NMR diffusometry in a hydrogel cylinder with $n$-hexane as bulk phase. This confirms that the presence of an organic solvent has no significant effect on the diffusion of DMBA in the hydrogel. The estimate for the diffusion coefficient of TMB deviates from the one obtained without MIBK. However, since the data quality of experiments with TMB was in general lower compared to DMBA due to the limited solubility and lower quantum yield of TMB, further investigations of the TMB diffusion are advisable. 
Finally, the complete gel-stabilized aqueous-organic biphasic reactive system was investigated. It was shown that in this system limitations due to diffusion or reaction need to be avoided. Line scans along the radius using two photon LSM were identified as the optimal measurement method. The derived kinetic model, considering all three occurring phenomena, describes the experimental data satisfactorily. Most model parameters were estimated with high precision. The parameter estimates were compared with those of the individual and coupled systems. No significant influence of the reaction system on the enzyme kinetic parameters was observed. The partition coefficients and the diffusion coefficient of the substrate are also practically independent of the reaction system. Significant deviations were only found for the mass transfer coefficients and the diffusion coefficient of the product. For this reason, these parameters have to be determined for each system separately.

Within this thesis it could be demonstrated that the MEXA approach is an excellent method to investigate complex reaction systems and to provide valuable information about kinetic mechanisms and possible limitations. Furthermore, this work highlighted the importance of the collaboration of experts from different scientific disciplines. Only the close and intensive cooperation made it possible to combine mechanistic kinetic modeling, optimal experimental design, and highly resolved measurement methods. However, the future goal should be that the MEXA method can also be applied by experimental researchers who have limited time and no expert knowledge for detailed mathematics and statistics. Thus, software tools assisting the user in applying the MEXA method should be developed. Furthermore, NMR should be established as measuring technique for studying enzyme immobilizates, since NMR allows the quantification of any organic substance.

Since the derived mechanistic kinetic model for the gel-stabilized aqueous-organic biphasic reaction system permits to easily detect limitations caused by diffusion or by mass transfer, it constitutes the basis for the rational design of enzyme immobilizates and for the optimization of such processes. Due to its modular structure the kinetic model can be adapted to other reaction systems, biocatalysts, geometries, and solvents. One option for further investigations is the application of the kinetic model to covalently immobilized enzymes.

It could be demonstrated using the examples of BAL and BFD that mechanistic kinetic modeling provides important information about the enzyme mechanism and allows for the identification of catalytic bottlenecks. For this reason, mechanistic kinetic modeling certainly 
will support the development of new enzyme variants with enhanced activities. In the future biocatalysis will be applied to efficiently convert large quantities of renewable resources into materials and biofuels. Hence, the optimization of biocatalysts and biocatalytic processes using mechanistic kinetic modeling will be of particular importance. 


\section{Bibliography}

Ahlrichs R, Bär M, Häser M, Horn H, Kölmel C. 1989. Electronic structure calculations on workstation computers: The program system turbomole. Chemical Physics Letters 162(3): 165-169.

Alberty RA. 2008. Determination of kinetic parameters of enzyme-catalyzed reactions with a minimum number of velocity measurements. Journal of Theoretical Biology 254(1): 156163.

Alexander C. 2008. Stimuli-responsive hydrogels: drugs take control. Nature Materials 7(10): 767-768.

Alkema WB, Floris R, Janssen DB. 1999. The use of chromogenic reference substrates for the kinetic analysis of penicillin acylases. Analytical Biochemistry 275(1): 47-53.

Alkema WBL, Hensgens CMH, Kroezinga EH, de Vries E, Floris R, van der Laan JM, Dijkstra BW, Janssen DB. 2000. Characterization of the beta-lactam binding site of penicillin acylase of Escherichia coli by structural and site-directed mutagenesis studies. Protein Engineering 13(12): 857-863.

Altman M, Gill J, McDonald MP. 2004. Numerical issues in statistical computing for the scientist. New York: John Wiley \& Sons.

Ansorge-Schumacher M. 2007. Two-phase systems with solidified water phases - tools for technical use of sensitive catalysts. Mini-Reviews in Organic Chemistry 4(3): 243-245.

Ansorge-Schumacher M, Doumeche B, Metrangolo D, Hartmeier W. 2000. Enzyme encapsulation for biocatalysis in organic solvents - new approaches to the stereoselective synthesis of pharmaceutical compounds. Minerva Biotechnologica 12: 265-269. 
Ansorge-Schumacher MB, Greiner L, Schroeper F, Mirtschin S, Hischer T. 2006. Operational concept for the improved synthesis of (R)-3,3'-furoin and related hydrophobic compounds with benzaldehyde lyase. Biotechnology Journal 1(5): 564-568.

Arellano-Garcia H, Schöneberger J, Körkel S. 2007. Optimale Versuchsplanung in der chemischen Verfahrenstechnik. Chemie Ingenieur Technik 79(10): 1625-1638.

Arroyo M, de la Mata I, Acebal C, Castillon MP. 2003. Biotechnological applications of penicillin acylases: state-of-the-art. Applied Microbiology and Biotechnology 60(5): 507514.

Asprey SP, Macchietto S. 2000. Statistical tools for optimal dynamic model building. Computers \& Chemical Engineering 24(2-7): 1261-1267.

Atkinson AC, Fedorov VV. 1975a. Design of experiments for discriminating between two rival models. Biometrika 62(1): 57-70.

Atkinson AC, Fedorov VV. 1975b. Optimal design - experiments for discriminating between several models. Biometrika 62(2): 289-303.

Baldascini H, Ganzeveld KJ, Janssen DB, Beenackers AACM. 2001. Effect of mass transfer limitations on the enzymatic kinetic resolution of epoxides in a two-liquid-phase system. Biotechnology and Bioengineering 73(1): 44-54.

Baldascini H, Janssen DB. 2005. Interfacial inactivation of epoxide hydrolase in a two-liquidphase system. Enzyme and Microbial Technology 36(2-3): 285-293.

Balkenhohl F, Ditrich K, Hauer B, Ladner W. 1997. Optisch aktive Amine durch Lipasekatalysierte Methoxyacetylierung. Journal für Praktische Chemie/Chemiker-Zeitung 339(1): 381-384.

Bard Y. 1974. Nonlinear parameter estimation. New York: Academic Press. 
Bardow A, Marquardt W. 2004a. Identification of diffusive transport by means of an incremental approach. Computers \& Chemical Engineering 28(5): 585-595.

Bardow A, Marquardt W. 2004b. Incremental and simultaneous identification of reaction kinetics: methods and comparison. Chemical Engineering Science 59(13): 2673-2684.

Bardow A, Marquardt W. 2007. Incremental identification methods for reaction and transport kinetics. Encyclopedia of Optimization. 2nd ed: Fludas, C. A.

Bates DJ, Frieden C. 1973. Treatment of enzyme kinetic data. 3. Use of full time course of a reaction, as examined by computer-simulation, in defining enzyme mechanisms. Journal of Biological Chemistry 248(22): 7878-7884.

Bauer I, Bock HG, Koerkel S, Schloeder JP. 2000: Numerical methods for optimum experimental design in DAE systems. Journal of Computational and Applied Mathematics 120(1-2): 1-25.

Bauer M, Geyer R, Griengl H, Steiner W. 2002. The use of lewis cell to investigate the enzyme kinetics of an $(S)$-hydroxynitrile lyase in two-Phase Systems. Food Technology and Biotechnology 40(1): 9-19.

Beck J, Arnold K. 1977. Parameter Estimation in Engineering and Science. New York: John Wiley and Sons Inc.

Berendsen WR, Lapin A, Reuss M. 2006. Investigations of reaction kinetics for immobilized enzymes - identification of parameters in the presence of diffusion limitation. Biotechnology Progress 22(5): 1305-1312.

Berger RJ, Stitt EH, Marin GB, Kapteijn F, Moulijn JA. 2001. Eurokin - Chemical reaction kinetics in practice. Cattech 5(1): 30-60.

Blaser HU. 2003. Enantioselective catalysis in fine chemicals production. Chemical Communications (Camb)(3): 293-296. 
Box GEP, Lucas HL. 1959. Design of experiments in non-linear situations. Biometrika 46(1): 77-90.

Bradford MM. 1976. A rapid and sensitive method for the quantitation of microgram quantities of protein utilizing the principle of protein-dye binding. Analytical Biochemistry 72: $248-254$.

Brendel M, Bonvin D, Marquardt W. 2006. Incremental identification of kinetic models for homogeneous reaction systems. Chemical Engineering Science 61(16): 5404-5420.

Breuer M, Ditrich K, Habicher T, Hauer B, Kesseler M, Stürmer R, Zelinski T. 2004. Industrial methods for the production of optically active intermediates. Angewandte Chemie International Edition 43(7): 788-824.

Bruggink A, Roos EC, de Vroom E. 1998. Penicillin acylase in the industrial production of beta-lactam antibiotics. Organic Process Research \& Development 2(2): 128-133.

Buchholz K. 1989. Immobilized enzymes - Kinetics, efficiency, and applications. Chemie Ingenieur Technik 61(8): 611-620.

Buchholz K, Kasche V, Bornscheuer U. 2005. Biocatalysts and enzyme technology. Weinheim: Wiley-VCH.

Burke AL, Duever TA, Penlidis A. 1997. Choosing the right model: Case studies on the use of statistical model discrimination experiments. Canadian Journal of Chemical Engineering 75(2): 422-436.

Buthe A, Hartmeier W, Ansorge-Schumacher MB. 2004. Novel solvent-based method for preparation of alginate beads with improved roundness and predictable size. Journal of Microencapsulation 21(8): 865-876.

Butler LG. 1979. Enzymes in non-aqueous solvents. Enzyme and Microbial Technology 1(4): 253-259. 
Cantone S, Hanefeld U, Basso A. 2007. Biocatalysis in non-conventional media-ionic liquids, supercritical fluids and the gas phase. Green Chemistry 9(9): 954-971.

Cao L. 2005. Carrier-bound immobilized enzymes - principles, applications and design. Weinheim: Wiley-VCH.

Cappuyns AM, Bernaerts K, Smets IY, Ona O, Prinsen E, Vanderleyden J, Van Impe JF. 2007. Optimal fed batch experiment design for estimation of monod kinetics of Azospirillum brasilense: From theory to practice. Biotechnology Progress 23(5): 10741081.

Chakraborty S, Nemeria N, Yep A, McLeish MJ, Kenyon GL, Jordan F. 2008. Mechanism of benzaldehyde lyase studied via thiamin diphosphate-bound intermediates and kinetic isotope effects. Biochemistry 47(12): 3800-3809.

Cho YK, Bailey JE. 1978. Immobilization of enzymes on activated carbon: Properties of immobilized glucoamylase, glucose oxidase, and gluconolactonase. Biotechnology and Bioengineering 20(10): 1651-1665.

Citra MJ. 1997. Modelmaker 3.0 for windows. Journal of Chemical Information and Computer Sciences 37(6): 1198-1200.

Cleland WW. 1963. Kinetics of enzyme-catalyzed reactions with 2 or more substrates or products. 1. Nomenclature and rate equations. Biochimica Et Biophysica Acta 67(1): 104137.

Cole M. 1969. Deacylation of acylamino compounds other than penicillins by cell-bound penicillin acylase of Escherichia Coli. Biochemical Journal 115(4): 733-739.

Copeland RA. 2000. Enzymes: A practical introduction to structure, mechanism, and data analysis. New York: Wiley-VCH. 387-390.

Cornish-Bowden A. 2001. Detection of errors of interpretation in experiments in enzyme kinetics. Methods 24(2): 181-190. 
Craven P, Wahba G. 1979. Smoothing noisy data with spline functions - estimating the correct degree of smoothing by the method of generalized cross-validation. Numerische Mathematik 31(4): 377-403.

Cremonesi P, Carrea G, Ferrara L, Antonini E. 1974. Enzymatic dehydrogenation of testosterone coupled to pyruvate reduction in a two-phase system. European Journal of Biochemistry 44(2): 401-405.

Cremonesi P, Carrea G, Ferrara L, Antonini E. 1975. Enzymatic preparation of betahydroxysteroids in a two-phase system. Biotechnology and Bioengineering 17(8): 11011108 .

Cremonesi P, Carrea G, Sportoletti G, Antonini E. 1973. Enzymatic dehydrogenation of steroids by beta-hydroxysteroid dehydrogenase in a two-phase system. Archives of Biochemistry and Biophysics 159(1): 7-10.

Cukier RI. 1984. Diffusion of Brownian spheres in semidilute polymer solutions. Macromolecules 17(2): 252-255.

Cvetkovic A, Straathof AJJ, Hanlon DN, van der Zwaag S, Krishna R, van der Wielen LAM. 2004. Quantifying anisotropic solute transport in protein crystals using 3-D laser scanning confocal microscopy visualization. Biotechnology and Bioengineering 86(4): 389-398.

de Boor C. 1978. A practical guide to splines. New York: Springer-Verlag. 235-276.

Demir AS, Dünnwald T, Iding H, Pohl M, Müller M. 1999. Asymmetric benzoin reaction catalyzed by benzoylformate decarboxylase. Tetrahedron: Asymmetry 10(24): 4769-4774.

Demir AS, Hamamci H, Sesenoglu O, Aydogan F, Capanoglu D, Neslihanoglu R. 2001a. Simple chemoenzymatic access to enantiopure pharmacologically interesting $(R)-2$ hydroxypropiophenones. Tetrahedron: Asymmetry 12(13): 1953-1956. 
Demir AS, Pohl M, Janzen E, Müller M. 2001b. Enantioselective synthesis of hydroxy ketones through cleavage and formation of acyloin linkage. Enzymatic kinetic resolution via C-C bond cleavage. Journal of the Chemical Society-Perkin Transactions 1(7): 633635.

Denk W, Strickler J, Webb W. 1990. Two-photon laser scanning fluorescence microscopy. Science 248(4951): 73-76.

Diender MB, Straathof AJJ, van der Does T, Ras C, Heijnen JJ. 2002. Equilibrium modeling of extractive enzymatic hydrolysis of penicillin $\mathrm{G}$ with concomitant 6-aminopenicillanic acid crystallization. Biotechnology and Bioengineering 78(4): 395-402.

Dingee JW, Anton AB. 2008. A new perturbation solution to the Michaelis-Menten problem. AIChe Journal 54(5): 1344-1357.

Dominguez de Maria P, Stillger T, Pohl M, Wallert S, Drauz K, Gröger H, Trauthwein H, Liese A. 2006. Preparative enantio selective synthesis of benzoins and (R)-2-hydroxy-1phenylpropanone using benzaldehyde lyase. Journal of Molecular Catalysis B-Enzymatic 38(1): 43-47.

Draper NR, Hunter WG. 1966. Design of experiments for parameter estimation in multiresponse situations. Biometrika 53(3-4): 525-533.

Duggleby HJ, Tolley SP, Hill CP, Dodson EJ, Dodson G, Moody PCE. 1995. Penicillin acylase has a single-amino-acid catalytic center. Nature 373(6511): 264-268.

Duggleby RG. 2001. Quantitative analysis of the time courses of enzyme-catalyzed reactions. Methods 24(2): 168-174.

Duggleby RG, Clarke RB. 1991. Experimental designs for estimating the parameters of the Michaelis-Menten equation from progress curves of enzyme-catalyzed reactions. Biochimica et Biophysica Acta (BBA) - Protein Structure and Molecular Enzymology 1080(3): 231-236. 
Duggleby RG, Daniel LP. 1995. Analysis of enzyme progress curves by nonlinear regression. Methods in Enzymology: Academic Press. 61-90.

Dünkelmann P, Kolter-Jung D, Nitsche A, Demir AS, Siegert P, Lingen B, Baumann M, Pohl M, Müller M. 2002. Development of a donor-acceptor concept for enzymatic crosscoupling reactions of aldehydes: The first asymmetric cross-benzoin condensation. Journal of the American Chemical Society 124(41): 12084-12085.

Eckert F. 2006. COSMOtherm Users Manual, Version C2.1 Release 01.06, COSMOlogic $\mathrm{GmbH}$ and Co KG.

Eckstein MF, Peters M, Lembrecht J, Spiess AC, Greiner L. 2006a. Maximise equilibrium conversion in biphasic catalysed reactions: Mathematical description and practical guideline. Advanced Synthesis \& Catalysis 348(12-13): 1591-1596.

Eckstein MF, Lembrecht J, Schumacher J, Eberhard W, Spiess AC, Peters M, Roosen C, Greiner L, Leitner W, Kragl U. 2006b. Maximise equilibrium conversion in biphasic catalysed reactions: How to obtain reliable data for equilibrium constants? Advanced Synthesis \& Catalysis 348(12-13): 1597-1604.

Faber K, Kroutil W. 2005. New enzymes for biotransformations. Current Opinion in Chemical Biology 9(2): 181-187.

Fernandes P, Cabral JMS. 2008. Biocatalysis in biphasic systems: General. In: Carrea G, Riva S, editors. Organic Synthesis with Enzymes in Non-Aqueous Media. Weinheim: WileyVCH. 191-207.

Franceschini G. 2007. New formulations for model-based experiment design and application to a biodiesel production process. Dissertation: Imperial College London.

Franceschini G, Macchietto S. 2007. Validation of a model for biodiesel production through model-based experiment design. Industrial \& Engineering Chemistry Research 46(1): 220232. 
Franceschini G, Macchietto S. 2008a. Model-based design of experiments for parameter precision: State of the art. Chemical Engineering Science 63(19): 4846-4872.

Franceschini G, Macchietto S. 2008b. Anti-correlation approach to model-based experiment design: Application to a biodiesel production process. Industrial \& Engineering Chemistry Research 47(7): 2331-2348.

Franceschini G, Macchietto S. 2008c. Novel anticorrelation criteria for model-based experiment design: Theory and formulations. AIChe Journal 54(4): 1009-1024.

Franceschini G, Macchietto S. 2008d. Novel anticorrelation criteria for design of experiments: Algorithm and application. AIChe Journal 54(12): 3221-3238.

Franco A, García C, Carrasco EF, Roca E. 2008. Explaining the enhanced performance of pulsed bioreactors by mechanistic modeling. AIChe Journal 54(5): 1377-1387.

Franco R, Aran JM, Canela EI. 1991. Fitting integrated enzyme rate-equations to progress curves with the use of a weighting matrix. Biochemical Journal 274: 509-511.

Frazier BL, Larmour P, Riley MR. 2001. Noninvasive measurement of effective diffusivities in cell immobilization gels through use of near-infrared spectroscopy. Biotechnology and Bioengineering 72(3): 364-368.

Fukushima E. 1999. Nuclear magnetic resonance as a tool to study flow. Annual Review of Fluid Mechanics 31(1): 95-123.

Galunsky B, Schlothauer RC, Bockle B, Kasche V. 1994. Direct spectrophotometric measurement of enzyme-activity in heterogeneous systems with insoluble substrate or immobilized enzyme. Analytical Biochemistry 221(1): 213-214.

Galvanin F, Macchietto S, Bezzo F. 2007. Model-based design of parallel experiments. Industrial \& Engineering Chemistry Research 46(3): 871-882. 
Gao P, Fagerness PE. 1995. Diffusion in Hpmc Gels. 1. Determination of drug and water diffusivity by pulsed-field-gradient spin-echo NMR. Pharmaceutical Research 12(7): 955964.

Gerrits PJ, Willeman WF, Straathof AJJ, Heijnen JJ, Brussee J, van der Gen A. 2001. Mass transfer limitation as a tool to enhance the enantiomeric excess in the enzymatic synthesis of chiral cyanohydrins. Journal of Molecular Catalysis B: Enzymatic 15(4-6): 111-121.

Giordano RC, Ribeiro MPA, Giordano RLC. 2006. Kinetics of beta-lactam antibiotics synthesis by penicillin G acylase (PGA) from the viewpoint of the industrial enzymatic reactor optimization. Biotechnology Advances 24(1): 27-41.

Goncalves L, Ferreira A, Fernandez-Lafuente R, Guisan J, Giordano R. 2008. Influence of mass transfer limitations on the enzymatic synthesis of $\beta$-lactam antibiotics catalyzed by penicillin G acylase immobilized on glioxil-agarose. Bioprocess and Biosystems Engineering 31(5): 411-418.

Goncalves LRB, Sousa R, Fernandez-Lafuente R, Guisan JM, Giordano RLC, Giordano RC. 2002. Enzymatic synthesis of amoxicillin: Avoiding limitations of the mechanistic approach for reaction kinetics. Biotechnology and Bioengineering 80(6): 622-631.

Gonzalez B, Olave I, Calderon I, Vicuna R. 1988. Degradation of diarylethane structures by Pseudomonas fluorescens biovar I. Archives of Microbiology 149(5): 389-394.

Gonzalez B, Vicuna R. 1989. Benzaldehyde lyase, a novel thiamine Ppi-requiring enzyme, from Pseudomonas-Fluorescens Biovar-I. Journal of Bacteriology 171(5): 2401-2405.

Gröger H, Hummel W, Rollmann C, Chamouleau F, Hüsken H, Werner H, Wunderlich C, Abokitse K, Drauz K, Buchholz S. 2004. Preparative asymmetric reduction of ketones in a biphasic medium with an (S)-alcohol dehydrogenase under in situ-cofactor-recycling with a formate dehydrogenase. Tetrahedron 60(3): 633-640. 
Guisan JM, Alvaro G, Rosell CM, Fernandez-Lafuente R. 1994. Industrial design of enzymic processes catalysed by very active immobilized derivatives: utilization of diffusional limitations (gradients of $\mathrm{pH}$ ) as a profitable tool in enzyme engineering. Biotechnology and Applied Biochemistry 20: 357-369.

Gutierrez OA, Danielson UH. 2006a. Detection of competitive enzyme inhibition with end point progress curve data. Analytical Biochemistry 358(1): 11-19.

Gutierrez OA, Danielson UH. 2006b. Sensitivity analysis and error structure of progress curves. Analytical Biochemistry 358(1): 1-10.

Hadamard J. 1923. Lectures on Cauchy's problem in linear differential equations. New Haven: Yale University Press.

Halling PJ. 1987. Biocatalysis in multi-phase reaction mixtures containing organic liquids. Biotechnology Advances 5(1): 47-84.

Halling PJ. 1994. Thermodynamic predictions for biocatalysis in nonconventional media: Theory, tests, and recommendations for experimental design and analysis. Enzyme and Microbial Technology 16(3): 178-206.

Handrikova G, Stefuca V, Polakovic M, Báles V. 1996. Determination of effective diffusion coefficient of substrate in gel particles with immobilized biocatalyst. Enzyme and Microbial Technology 18(8): 581-584.

Hansen PC. 1992. Analysis of discrete ill-posed problems by means of the L-curve. Siam Review 34(4): 561-580.

Hansen PC. 1994. Regularization tools: A Matlab package for analysis and solution of discrete ill-posed problems. Numerical Algorithms 6: 1-35.

Hansen PC. 1998. Rank-deficient and discrete ill-posed problems. Philadelphia: SIAM. 
Hansen PC. 1999. Regularization tools version 3.0 for Matlab 5.2. Numerical Algorithms 20(2-3): 195-196.

Häser M, Ahlrichs R. 1989. Improvements on the direct SCF method. Journal of Computational Chemistry 10(1): 104-111.

Heinemann M. 2003. Experimental analysis, modeling and dynamic simulation of thermodynamic and kinetic phenomena in gel-stabilized enzyme carriers. Dissertation: RWTH Aachen University.

Heinemann M, Kümmel A, Giesen R, Ansorge-Schumacher MB, Büchs J. 2003. Experimental and theoretical analysis of phase equilibria in a two-phase system used for biocatalytic esterifications. Biocatalysis and Biotransformation 21(3): 115-121.

Heinemann M, Limper U, Büchs J. 2004. New insights in the spatially resolved dynamic pH measurement in macroscopic large absorbent particles by confocal laser scanning microscopy. Journal of Chromatography A 1024(1-2): 45-53.

Heinemann M, Meinberg H, Büchs J, Koss HJ, Ansorge-Schumacher MB. 2005. Method for quantitative determination of spatial polymer distribution in alginate beads using Raman spectroscopy. Applied Spectroscopy 59(3): 280-285.

Hildebrand F, Kühl S, Pohl M, Vasic-Racki D, Müller M, Wandrey C, Lütz S. 2007. The production of $(R)$-2-hydroxy-1-phenyl-propan-1-one derivatives by benzaldehyde lyase from Pseudomonas fluorescens in a continuously operated membrane reactor. Biotechnology and Bioengineering 96(5): 835-843.

Hill PDH. 1978. Review of experimental-design procedures for regression-model discrimination. Technometrics 20(1): 15-21.

Hischer T, Gocke D, Fernandez M, Hoyos P, Alcantara AR, Sinisterra JV, Hartmeier W, Ansorge-Schumacher MB. 2005. Stereoselective synthesis of novel benzoins catalysed by benzaldehyde lyase in a gel-stabilised two-phase system. Tetrahedron 61(31): 7378-7383. 
Horn H, Weiß H, Häser M, Ehrig M, Ahlrichs R. 1991. Prescreening of two-electron integral derivatives in SCF gradient and Hessian calculations. Journal of Computational Chemistry 12(9): 1058-1064.

Hunter WG, Reiner AM. 1965. Designs for discriminating between 2 rival models. Technometrics 7(3): 307-323.

Iding H, Dünnwald T, Greiner L, Liese A, Müller M, Siegert P, Grötzinger J, Demir AS, Pohl M. 2000. Benzoylformate decarboxylase from Pseudomonas putida as stable catalyst for the synthesis of chiral 2-hydroxy ketones. Chemistry A European Journal 6(8): 1483-1495.

Janzen E, Müller M, Kolter-Jung D, Kneen MM, McLeish MJ, Pohl M. 2006. Characterization of benzaldehyde lyase from Pseudomonas fluorescens: A versatile enzyme for asymmetric C-C bond formation. Bioorganic Chemistry 34(6): 345-361.

Jeong JJ, Fushinobu S, Ito S, Jeon BS, Shoun H, Wakagi T. 2003. Characterization of the cupin-type phosphoglucose isomerase from the hyperthermophilic archaeon Thermococcus litoralis. FEBS Letters 535(1-3): 200-204.

Jordan F. 2003. Current mechanistic understanding of thiamin diphosphatedependent enzymatic reactions. Natural Product Reports 20(2): 184-201.

Kalman RE. 1960. A new approach to linear filtering and prediction problems. Journal of Basic Engineering 82(1): 35-45.

Kasche V, Galunsky B, Ignatova Z. 2003. Fragments of pro-peptide activate mature penicillin amidase of Alcaligenes faecalis. European Journal of Biochemistry 270(23): 4721-4728.

Kheirolomoom A, Ardjmand M, Fazelinia H, Zakeri A. 2001. Clarification of penicillin G acylase reaction mechanism. Process Biochemistry 36(11): 1095-1101.

King EL, Altman C. 1956. A schematic method of deriving the rate laws for enzymecatalyzed reactions. Journal of Physical Chemistry 60(10): 1375-1378. 
Klamt A, Schüürmann G. 1993. COSMO: A new approach to dielectric screening in solvents with explicit expressions for the screening energy and its gradient. Journal of the Chemical Society-Perkin Transactions 2(5): 799-805.

Klibanov AM. 2001. Improving enzymes by using them in organic solvents. Nature 409(6817): 241-246.

Klibanov AM, Samokhin GP, Martinek K, Berezin IV. 1977. A new approach to preparative enzymatic synthesis. Biotechnology and Bioengineering 19(9): 1351-1361.

Kluger R. 1987. Thiamin diphosphate - a mechanistic update on enzymatic and nonenzymic catalysis of decarboxylation. Chemical Reviews 87(5): 863-876.

Klyachko NL, Levashov AV. 2003. Bioorganic synthesis in reverse micelles and related systems. Current Opinion in Colloid \& Interface Science 8(2): 179-186.

Knoll M, Müller M, Pleiss J, Pohl M. 2006. Factors mediating activity, selectivity, and substrate specificity for the thiamin diphosphate-dependent enzymes benzaldehyde lyase and benzoylformate decarboxylase. Chembiochem 7(12): 1928-1934.

Kopecek J, Yang J. 2007. Hydrogels as smart biomaterials. Polymer International 56(9): 1078-1098.

Kragl U, Eckstein M, Kaftzik N. 2002. Enzyme catalysis in ionic liquids. Current Opinion in Biotechnology 13(6): 565-571.

Kremling A, Fischer S, Gadkar K, Doyle FJ, Sauter T, Bullinger E, Allgöwer F, Gilles ED. 2004. A benchmark for methods in reverse engineering and model discrimination: Problem formulation and solutions. Genome Research 14(9): 1773-1785.

Küppers M, Heine C, Han S, Stapf S, Blümich B. 2002. In situ observation of diffusion and reaction dynamics in gel microreactors by chemically resolved NMR microscopy. Applied Magnetic Resonance 22(2): 235-246. 
Kurlemann N, Liese A. 2004. Immobilization of benzaldehyde lyase and its application as a heterogeneous catalyst in the continuous synthesis of a chiral 2-hydroxy ketone. Tetrahedron: Asymmetry 15(18): 2955-2958.

Kuwana E, Liang F, Sevick-Muraca EM. 2004. Fluorescence lifetime spectroscopy of a pHsensitive dye encapsulated in hydrogel beads. Biotechnology Progress 20(5): 1561-1566.

Kwak S, Lafleur M. 2003. Raman spectroscopy as a tool for measuring mutual-diffusion coefficients in hydrogels. Applied Spectroscopy 57(7): 768-773.

Kwak S, Viet MTP, Lafleur M. 2003. Self- and mutual-diffusion coefficients measurements by P-31 NMR ID profiling and PFG-SE in dextran gels. Journal of Magnetic Resonance 162(1): 198-205.

Laane C, Boeren S, Vos K, Veeger C. 1987. Rules for optimization of biocatalysis in organic solvents. Biotechnology and Bioengineering 30(1): 81-87.

Lamare S, Legoy M-D, Graber M. 2004. Solid/gas bioreactors: powerful tools for fundamental research and efficient technology for industrial applications. Green Chemistry 6: $445-458$.

Lawrence MJ, Rees GD. 2000. Microemulsion-based media as novel drug delivery systems. Advanced Drug Delivery Reviews 45(1): 89-121.

Leeper FJ, Hawksley D, Mann S, Melero CP, Wood MDH. 2005. Studies on thiamine diphosphate-dependent enzymes. Biochemical Society Transactions 33: 772-775.

Leresche JE, Meyer H-P. 2006. Chemocatalysis and biocatalysis (biotransformation): Some thoughts of a chemist and of a biotechnologist. Organic Process Research \& Development 10(3): 572-580.

Levenspiel O. 1972. Chemical reaction engineering. New York: Wiley-VCH. 
Lewis WK, Whitman WG. 1924. Principles of gas absorption. Industrial \& Engineering Chemistry 16(12): 1215-1220.

Liao F, Zhu XY, Wang YM, Zuo YP. 2005. The comparison of the estimation of enzyme kinetic parameters by fitting reaction curve to the integrated Michaelis-Menten rate equations of different predictor variables. Journal of Biochemical and Biophysical Methods 62(1): 13-24.

Lindner PFO, Hitzmann B. 2006. Experimental design for optimal parameter estimation of an enzyme kinetic process based on the analysis of the Fisher information matrix. Journal of Theoretical Biology 238(1): 111-123.

Lineweaver H. 1934. The determination of enzyme dissociation-constants. Journal of the American Chemical Society 56(3): 658-666.

Magario I, Ma X, Neumann A, Syldatk C, Hausmann R. 2008. Non-porous magnetic microparticles: Comparison to porous enzyme carriers for a diffusion rate-controlled enzymatic conversion. Journal of Biotechnology 134(1-2): 72-78.

Malmborg C, Topgaard D, Söderman O. 2003. Diffusion in an inhomogeneous system: NMR studies of diffusion in highly concentrated emulsions. Journal of Colloid and Interface Science 263(1): 270-276.

Markus M, Plesser T, Kohlmeier M. 1981. Analysis of progress curves in enzyme-kinetics bias and convergent set in the differential and in the integral method. Journal of Biochemical and Biophysical Methods 4(2): 81-90.

Marokhazi J, Koczan G, Hudecz F, Graf L, Fodor A, Venekei I. 2004. Enzymic characterization with progress curve analysis of a collagen peptidase from an enthomopathogenic bacterium, Photorhabdus luminescens. Biochemical Journal 379: 633640.

Marquardt W. 2005. Model-based experimental analysis of kinetic phenomena in multi-phase reactive systems. Chemical Engineering Research \& Design 83(A6): 561-573. 
Martinsen A, Storro I, Skjakbraek G. 1992. Alginate as immobilization material. 3. Diffusional properties. Biotechnology and Bioengineering 39(2): 186-194.

Masaro L, Zhu XX, MacDonald PM. 1999. Study of the self-diffusion of poly(ethylene glycol)s in poly(vinyl alcohol) aqueous systems. Journal of Polymer Science Part BPolymer Physics 37(17): 2396-2403.

Mateo C, Palomo JM, Fernandez-Lorente G, Guisan JM, Fernandez-Lafuente R. 2007. Improvement of enzyme activity, stability and selectivity via immobilization techniques. Enzyme and Microbial Technology 40(6): 1451-1463.

Mateus DMR, Alves SS, da Fonseca MMR. 1999. Diffusion in cell-free and cell immobilising kappa-carrageenan gel beads with and without chemical reaction. Biotechnology and Bioengineering 63(5): 625-631.

McCullough BD. 1998. Assessing the reliability of statistical software: Part I. The American Statistician 52(4): 358-366.

McCullough BD. 1999. Assessing the reliability of statistical software: Part II. The American Statistician 53(2): 149-159.

McCullough BD, Wilson B. 1999. On the accuracy of statistical procedures in Microsoft Excel 97. Computational Statistics \& Data Analysis 31(1): 27-37.

Metrangolo-Ruiz de Temiño D, Hartmeier W, Ansorge-Schumacher MB. 2005. Entrapment of the alcohol dehydrogenase from Lactobacillus kefir in polyvinyl alcohol for the synthesis of chiral hydrophobic alcohols in organic solvents. Enzyme and Microbial Technology 36(1): 3-9.

Michaelis L, Menten M. 1913. Die Kinetik der Invertinwirkung. Biochemische Zeitschrift 49: 333-369.

Michalik C, Brendel M, Marquardt W. 2009. Incremental identification of fluid multi-phase reaction systems. AIChe Journal 55(4): 1009-1022. 
Michalik C, Schmidt T, Zavrel M, Ansorge-Schumacher M, Spiess A, Marquardt W. 2007. Application of the incremental identification method to the formate oxidation using formate dehydrogenase. Chemical Engineering Science 62(18-20): 5592-5597.

Mikolajek R, Spiess AC, Pohl M, Lamare S, Büchs J. 2007. An activity, stability and selectivity comparison of propioin synthesis by thiamine diphosphate-dependent enzymes in a solid/gas bioreactor. Chembiochem 8(9): 1063-1070.

Mikolajek RJ, Spiess AC, Pohl M, Büchs J. 2009. Propioin synthesis using thiamine diphosphate-dependent enzymes. Biotechnology Progress 25(1): 132-138.

Mollerup J, Hansen E. 1998. Overall mass-transfer coefficients in non-linear chromatography. Journal of Chromatography A 827(2): 235-239.

Mosbacher TG, Müller M, Schulz GE. 2005. Structure and mechanism of the ThDPdependent benzaldehyde lyase from Pseudomonas fluorescens. FEBS Journal 272(23): 6067-6076.

Muhr AH, Blanshard JMV. 1982. Diffusion in gels. Polymer 23(7): 1012-1026.

Murphy EF, Gilmour SG, Crabbe MJC. 2003. Efficient and accurate experimental design for enzyme kinetics: Bayesian studies reveal a systematic approach. Journal of Biochemical and Biophysical Methods 55(2): 155-178.

Newman PFJ, Atkins GL, Nimmo IA. 1974. Effect of systematic-error on accuracy of Michaelis constants and maximum velocities estimated by using integrated MichaelisMenten equation. Biochemical Journal 143(3): 779-781.

Peters M, Eckstein MF, Hartjen G, Spiess AC, Leitner W, Greiner L. 2007. Exploring conversion of biphasic catalytic reactions: Analytical solution and parameter study. Industrial \& Engineering Chemistry Research 46(22): 7073-7078.

Pohl M, Lingen B, Müller M. 2002. Thiamin-diphosphate-dependent enzymes: New aspects of asymmetric C-C bond formation. Chemistry A European Journal 8(23): 5289-5295. 
Polakovic M, Kudlacova G, Stefuca V, Bales V. 2001. Determination of sucrose effective diffusivity and intrinsic rate constant of hydrolysis catalysed by $\mathrm{Ca}$-alginate entrapped cells. Chemical Engineering Science 56(2): 459-466.

Rairkar M, Hayes D, Harris J. 2007. Solubilization of enzymes in water-in-oil microemulsions and their rapid and efficient release through use of a $\mathrm{pH}$-degradable surfactant. Biotechnology Letters 29(5): 767-771.

Rakels JLL, Romein B, Straathof AJJ, Heijnen JJ. 1994. Kinetic-analysis of enzymatic chiral resolution by progress curve evaluation. Biotechnology and Bioengineering 43(5): 411422.

Reinsch CH. 1967. Smoothing by spline functions. Numerische Mathematik 10(3): 177-183.

Reynolds LJ, Garcia GA, Kozarich JW, Kenyon GL. 1988. Differential reactivity in the processing of [para-(halomethyl)benzoyl]formates by benzoylformate decarboxylase, a thiamin pyrophosphate dependent enzyme. Biochemistry 27(15): 5530-5538.

Rosche B, Breuer M, Hauer B, Rogers PL. 2004. Biphasic aqueous/organic biotransformation of acetaldehyde and benzaidehyde by Zymomonas mobilis pyruvate decarboxylase. Biotechnology and Bioengineering 86(7): 788-794.

Ryder AG, Power S, Glynn TJ. 2003. Fluorescence lifetime based pH sensing using resorufin. In: Glynn TJ, editor. Opto-Ireland 2002: Optics and Photonics Technologies and Applications. Bellingham: SPIE. 827-835.

Sakaguchi K, Murao S. 1950. A preliminary report on a new enzyme "penicillin-amidase". Journal of the Agricultural Chemical Society of Japan 23: 410-411.

Salvati A, Lynch I, Malmborg C, Topgaard D. 2007. Chemical shift imaging of molecular transport in colloidal systems: Visualization and quantification of diffusion processes. Journal of Colloid and Interface Science 308(2): 542-550. 
Santos ETF, Bassrei A. 2007. L- and [Theta]-curve approaches for the selection of regularization parameter in geophysical diffraction tomography. Computers \& Geosciences 33(5): 618-629.

Schmid A, Dordick JS, Hauer B, Kiener A, Wubbolts M, Witholt B. 2001. Industrial biocatalysis today and tomorrow. Nature 409(6817): 258-268.

Schmid A, Hollmann F, Park JB, Bühler B. 2002. The use of enzymes in the chemical industry in Europe. Current Opinion in Biotechnology 13(4): 359-366.

Schmidt T. 2008. Experimentelle Analyse, Modellierung und biochemische Charakterisierung von Ein- und Zweiphasenreaktionen für die technische Biokatalyse. Dissertation: RWTH Aachen University.

Schoemaker HE, Mink D, Wubbolts MG. 2003. Dispelling the myths - biocatalysis in industrial synthesis. Science 299(5613): 1694-1697.

Schroën CGPH, Fretz CB, DeBruin VH, Berendsen WR, Moody HM, Roos EC, VanRoon JL, Kroon PJ, Strubel M, Janssen AEM and others. 2002. Modelling of the enzymatic kinetically controlled synthesis of cephalexin: Influence of diffusion limitation. Biotechnology and Bioengineering 80(3): 331-340.

Schulze B, Wubbolts MG. 1999. Biocatalysis for industrial production of fine chemicals. Current Opinion in Biotechnology 10(6): 609-615.

Seland JG, Ottaviani M, Hafskjold B. 2001. A PFG-NMR study of restricted diffusion in heterogeneous polymer particles. Journal of Colloid and Interface Science 239(1): 168177.

Selwyn MJ. 1965. A simple test for inactivation of an enzyme during assay. Biochimica Et Biophysica Acta 105(1): 193-195.

Sheldon RA. 2005. Green solvents for sustainable organic synthesis: State of the art. Green Chemistry 7: 267-278. 
Sheldon RA. 2007. Enzyme immobilization: The quest for optimum performance. Advanced Synthesis \& Catalysis 349(8-9): 1289-1307.

Skjak-Braek G, Grasdalen H, Smidsrod O. 1989. Inhomogeneous polysaccharide ionic gels. Carbohydrate Polymers 10(1): 31-54.

Spiess A, Schlothauer RC, Hinrichs J, Scheidat B, Kasche V. 1999. pH gradients in immobilized amidases and their influence on rates and yields of beta-lactam hydrolysis. Biotechnology and Bioengineering 62(3): 267-277.

Spiess AC, Eberhard W, Peters M, Eckstein MF, Greiner L, Büchs J. 2008a. Prediction of partition coefficients using COSMO-RS: Solvent screening for maximum conversion in biocatalytic two-phase reaction systems. Chemical Engineering and Processing: Process Intensification 47(6): 1034-1041.

Spiess AC, Kasche V. 2001. Direct measurement of $\mathrm{pH}$ profiles in immobilized enzyme carriers during kinetically controlled synthesis using CLSM. Biotechnology Progress 17(2): 294-303.

Spiess AC, Zavrel M, Ansorge-Schumacher MB, Janzen C, Michalik C, Schmidt TW, Schwendt T, Büchs J, Poprawe R, Marquardt W. 2008b. Model discrimination for the propionic acid diffusion into hydrogel beads using lifetime confocal laser scanning microscopy. Chemical Engineering Science 63(13): 3457-3465.

Stillger T, Pohl M, Wandrey C, Liese A. 2006. Reaction engineering of benzaldehyde lyase from Pseudomonas fluorescens catalyzing enantioselective C-C bond formation. Organic Process Research \& Development 10(6): 1172-1177.

Straathof AJJ. 2001. Development of a computer program for analysis of enzyme kinetics by progress curve fitting. Journal of Molecular Catalysis B: Enzymatic 11(4-6): 991-998.

Straathof AJJ. 2003. Enzymatic catalysis via liquid-liquid interfaces. Biotechnology and Bioengineering 83(4): 371-375. 
Straathof AJJ, Heijnen JJ. 1996. New constraints between kinetic parameters explain the (un)identifiability of enzymatic rate constants. Biotechnology and Bioengineering 52(3): 433-437.

Straathof AJJ, Panke S, Schmid A. 2002. The production of fine chemicals by biotransformations. Current Opinion in Biotechnology 13(6): 548-556.

Svedas VK, Margolin AL, Sherestyuk CF, Klyosov AA, Berezin IV. 1977. Phenylmethansulfonylfloride inactivation of soluble and immobilized pernicillin amidase from E. coli. Biotechnology Letters 7: 877-882.

Tallarek U, Rapp E, Sann H, Reichl U, Seidel-Morgenstern A. 2003. Quantitative study of electrokinetic transport in porous media by confocal laser scanning microscopy. Langmuir 19(11): 4527-4531.

Tanaka H, Matsumura M, Veliky IA. 1984. Diffusion characteristics of substrates in Caalginate gel beads. Biotechnology and Bioengineering 26(1): 53-58.

Taylor R, Krishna R. 1993. Multicomponent mass transfer. New York: Wiley.

Tellinghuisen J. 2001. Statistical error propagation. Journal of Physical Chemistry A 105(15): $3917-3921$.

Thu B, Gaserod O, Paus D, Mikkelsen A, Skjak-Braek G, Toffanin R, Vittur F, Rizzo R. 2000. Inhomogeneous alginate gel spheres: An assessment of the polymer gradients by synchrotron radiation-induced x-ray emission, magnetic resonance microimaging, and mathematical modeling. Biopolymers 53(1): 60-71.

Tittmann K, Golbik R, Uhlemann K, Khailova L, Schneider G, Patel M, Jordan F, Chipman DM, Duggleby RG, Hübner G. 2003. NMR analysis of covalent intermediates in thiamin diphosphate enzymes. Biochemistry 42(26): 7885-7891.

Treutler O, Ahlrichs R. 1995. Efficient molecular numerical integration schemes. The Journal of Chemical Physics 102(1): 346-354. 
Trusek-Holownia A. 2003. A membrane phase contactor for enzymatic synthesis of ZAlaPheOMe, the precursor of bitter dipeptide. Biochemical Engineering Journal 16(1): 69-77.

Trusek-Holownia A, Noworyta A. 2000. Dipeptide enzymatic synthesis in a two-phase membrane reactor. Chemical Papers 54(6b): 442-447.

van Roon J, Arntz M, Kallenberg A, Paasman M, Tramper J, Schroën C, Beeftink H. 2006. A multicomponent reaction-diffusion model of a heterogeneously distributed immobilized enzyme. Applied Microbiology and Biotechnology 72(2): 263-278.

van Roon J, Beeftink R, Schroën K, Tramper H. 2002. Assessment of intraparticle biocatalytic distributions as a tool in rational formulation. Current Opinion in Biotechnology 13(4): 398-405.

van Roon JL, Groenendijk E, Kieft H, Schroën CGPH, Tramper J, Beeftink HH. 2005. Novel approach to quantify immobilized-enzyme distributions. Biotechnology and Bioengineering 89(6): 660-669.

van Roon JL, Joerink M, Rijkers MPWM, Tramper J, Schroën CGPH, Beeftink HH. 2003. Enzyme distribution derived from macroscopic particle behavior of an industrial immobilized penicillin-G acylase. Biotechnology Progress 19(5): 1510-1518.

Vasic-Racki D, Kragl U, Liese A. 2003. Benefits of enzyme kinetics modelling. Chemical and Biochemical Engineering Quarterly 17(1): 7-18.

Vehreschild A. 2004. The ADiMat handbook: Institute for Scientifc Computing, RWTH Aachen University.

Villela Filho M, Stillger T, Müller M, Liese A, Wandrey C. 2003. Is $\log P$ a convenient criterion to guide the choice of solvents for biphasic enzymatic reactions? Angewandte Chemie 115(26): 3101-3104. 
Vrsalovic Presecki A, Findrik Z, Zelic B. 2006. Modeling of the biotransformation processes. Chemical and Biochemical Engineering Quarterly 20(3): 227-241.

Wahba G. 1990. Spline models for observational data. Philadelphia: SIAM.

Walter E, Pronzato L. 1990. Qualitative and quantitative experiment design for phenomenological models - a survey. Automatica 26(2): 195-213.

Warburton D, Dunnill P, Lilly MD. 1973. Conversion of benzylpenicillin to 6aminopenicillanic acid in a batch reactor and continuous feed stirred tank reactor using immobilized penicillin amidase. Biotechnology and Bioengineering 15(1): 13-25.

Weiss PM, Garcia GA, Kenyon GL, Cleland WW, Cook PF. 1988. Kinetics and mechanism of benzoylformate decarboxylase using $\mathrm{C}-13$ and solvent deuterium-isotope effects on benzoylformate and benzoylformate analogs. Biochemistry 27(6): 2197-2205.

Wilcocks R, Ward OP, Collins S, Dewdney NJ, Hong Y, Prosen E. 1992. Acyloin formation by benzoylformate decarboxylase from Pseudomonas putida. Applied and Environment Microbiology 58(5): 1699-1704.

Willaert RG, Baron GV. 1996. Gel entrapment and micro-encapsulation: Methods, applications and engineering principles. London: Freund Publishing House.

Willeman WF, Gerrits PJ, Hanefeld U, Brussee J, Straathof AJJ, van der Gen A, Heijnen JJ. 2002a. Development of a process model to describe the synthesis of $(R)$-mandelonitrile by Prunus amygdalus hydroxynitrile lyase in an aqueous-organic biphasic reactor. Biotechnology and Bioengineering 77(3): 239-247.

Willeman WF, Neuhofer R, Wirth I, Pochlauer P, Straathof AJJ, Heijnen JJ. 2002b. Development of $(R)$-4-hydroxymandelonitrile synthesis in an aqueous-organic biphasic stirred tank batch reactor. Biotechnology and Bioengineering 79(2): 154-164. 
Woodley JM. 2008. New opportunities for biocatalysis: making pharmaceutical processes greener. Trends in Biotechnology 26(6): 321-327.

Yablonsky GS, Constales D, Shekhtmanc SO, Gleaves JT. 2007. The Y-procedure: How to extract the chemical transformation rate from reaction-diffusion data with no assumption on the kinetic model. Chemical Engineering Science 62(23): 6754-6767.

Yeow YL, Pokethitiyook P, Cheah MY, Dang HDT, Law CKP. 2004. An alternative way of analyzing the progress curves of enzyme-catalyzed reactions. Biochemical Engineering Journal 21(1): 1-10.

Yeow YL, Wickramasinghe SR, Han B, Leong Y-K. 2003. A new method of processing the time-concentration data of reaction kinetics. Chemical Engineering Science 58(16): 36013610.

Zhong D. 2007. Ultrafast catalytic processes in enzymes. Current Opinion in Chemical Biology 11(2): 174-181. 


\section{Lebenslauf}

Name, Vorname

Geburtsdatum:

Geburtsort:

Staatsangehörigkeit:

\section{Schulausbildung}

1999

\section{Zivildienst}

1999-2000

Studium

2000-2005

2004

2004-2005

\section{Berufstätigkeit}

2005-2008

seit $12 / 2008$
Zavrel, Michael

21.04.1980

München

deutsch
Zivildienst beim Bayerischen Blinden- und

Sehbehindertenbund in München

Studium des Chemie-Ingenieurwesens an der

Technischen Universität München

Studienaufenthalt an der University of California in Santa Barbara, USA

Diplomarbeit bei der Roche Diagnostics $\mathrm{GmbH}$, Penzberg

Wissenschaftlicher Mitarbeiter am Lehrstuhl für Bioverfahrenstechnik der RWTH Aachen

Verfahrensingenieur bei der Süd-Chemie AG in München 